\title{
The Role of Clustered Organization and Generation of Mixed Properties in Macaque V2
}

\author{
Clinton Garret Cooper \\ West Virginia University
}

Follow this and additional works at: https://researchrepository.wvu.edu/etd

\section{Recommended Citation}

Cooper, Clinton Garret, "The Role of Clustered Organization and Generation of Mixed Properties in Macaque V2" (2012). Graduate Theses, Dissertations, and Problem Reports. 3581.

https://researchrepository.wvu.edu/etd/3581

This Dissertation is protected by copyright and/or related rights. It has been brought to you by the The Research Repository @ WVU with permission from the rights-holder(s). You are free to use this Dissertation in any way that is permitted by the copyright and related rights legislation that applies to your use. For other uses you must obtain permission from the rights-holder(s) directly, unless additional rights are indicated by a Creative Commons license in the record and/ or on the work itself. This Dissertation has been accepted for inclusion in WVU Graduate Theses, Dissertations, and Problem Reports collection by an authorized administrator of The Research Repository @ WVU.

For more information, please contact researchrepository@mail.wvu.edu. 


\title{
The Role of Clustered Organization and Generation of Mixed Properties in Macaque V2
}

\author{
Clinton Garret Cooper \\ Dissertation submitted to the \\ School of Medicine \\ at West Virginia University \\ in partial fulfillment of the requirements \\ for the degree of \\ Doctor of Philosophy \\ in \\ Neurobiology and Anatomy \\ Charles L. Rosen, M.D., Ph.D., Chair \\ Ariel Agmon, Ph.D. \\ James W. Lewis, Ph.D. \\ J. Vernon Odom, Ph.D. \\ Benjamin M. Ramsden, Ph.D. \\ Department of Neurobiology and Anatomy \\ Morgantown, West Virginia \\ 2012
}

Keywords: vision, V2, macaque, modeling, electrophysiology 


\section{Abstract \\ The Role of Clustered Organization and Generation of Mixed Properties in Macaque V2 \\ Clinton Garret Cooper}

Throughout the mammalian cortex, neurons of similar response characteristics group together into topographic functional domains. The genesis and role of this organization remains in question, but it has been proposed to affect the mixed properties of neurons. These types of neurons possess multiple receptive field preferences, such as a cell responding to a color and an oriented stimulus. To examine the functionality of clustered organization and their effect in generation of neurons possessing mixed properties, this dissertation examined the secondary visual cortex (V2) of the Macaca fasicularis. This particular cortex is comprised of domains organized according to distinct visual stimulus components, specifically clusters of neurons partitioned by color and orientation preferences within a close proximity. In the first series of experiments (Chapter 3), a computer model of a cortical area based upon macaque V2 investigated the effect of clusters of like-preferring neurons on the probability of two different preference terminals synapsing on a particular cell. These results indicate that presence of at least one cluster significantly increases the probability of multiple preferences arriving at a neuron. The second series of experiments (Chapter 4) used single unit electrophysiology to investigate the temporal properties of V2 neurons in response to achromatic and colored oriented stimuli. With the addition of color to the stimulus, an increase in latency, an increase to the time point of the maximum rate of firing, and a decreased initial-phase response with a sustained later-phase response were observed. These studies indicate that functional clusters of neurons significantly increase the joint probability of the co-localization of differing preference terminals, potentially yielding neurons with mixed preferences through these intra-areal connections. Furthermore, the temporal characteristics of V2 neurons, as seen in observed latency and time of maximum spiking, support this idea of domain-enhanced intra-areal integration. 


\section{Dedication}

This work is dedicated to the memory of my grandmother, Margaret Louise Shemasek Luketich. I love you, Mamie. 


\section{Acknowledgements}

I would like to acknowledge and thank my parents, William Clinton and Diane Louise Cooper, my brother, Grant, and my grandparents, John and LaVonne Cooper, for their constant support, encouragement and tolerance during my long journey through academia. I thank you and love you all.

I would also like to acknowledge and thank my advisor, Benjamin Ramsden, and all the members of my committee for their advice and guidance during my training. Likewise, I extend my gratitude to Bell Delaney, the lab’s manager, for all her help and friendship.

Finally, my thanks to all my colleagues I have had the privilege to work and collaborate with during my time at WVU. I am especially grateful to those who were there when all I needed was a friend to lend a guiding hand. 


\section{Table of Contents}

DEDICATION ................................................................................................................................................III

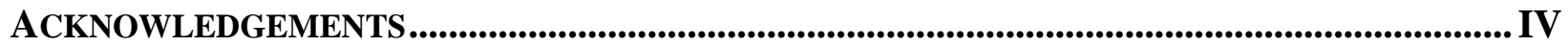

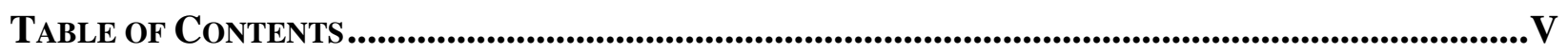

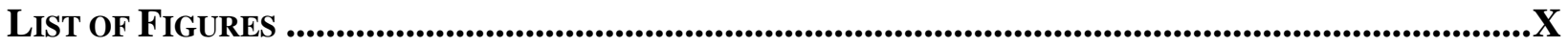

LIST OF ABBREVIATIONS .................................................................................................................

CHAPTER 1: INTRODUCTION AND LITERATURE REVIEW ..........................................................1

1.1 Overview of the Visual System: Retina to V1 ........................................................................2

1.1.1 Visual Processing and Neuronal Functional Organization: Retina to Lateral

Geniculate Nucleus ........................................................................................................

1.1.2 Visual Processing and Neuronal Functional Organization: Primary Visual Cortex...8

1.2 Overview of the Secondary Visual Cortex....................................................................14

1.2.1 Secondary Visual Cortex: Stripe Compartments and Functional Organization ........14

1.2.2 Secondary Visual Cortex: Stripe Connectivity ……………………………….........16

1.2.3 Secondary Visual Cortex: Stripe Input from V1 and Integration of Color and

Orientation Responses .........................................................................................................17

1.3 The Role of Clustered Organization in Cortical Sensory Processing ..................................20

1.4 Temporal Response Characteristics of V1 and V2: Latency .............................................22

1.4.1 Documented Latencies..........................................................................................22

1.4.2 Circuitry and Mechanisms Associated With Observed Latencies..............................24

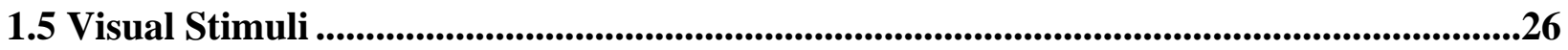

1.5.1 DLK Color Space..............................................................................................26

1.5.2 Linear vs. Non-Linear Processing in Visual Neurons ...............................................26

1.6 Sensory Processing Modeling.............................................................................................28

1.6.1 Previous Visual Cortex Models ................................................................................28

1.6.2 Common Design Features in Cortical Network Models............................................29

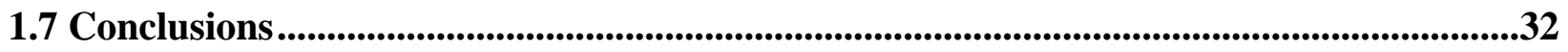

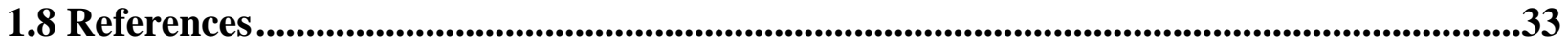


2.1 Objective 1 - Develop a biologically inspired computational network model to investigate and characterize the relationship between clustered functional organization and probability of convergent combinatorial circuitry within Area V2

2.2 Objective 2 - Compare and quantify the differential temporal responses associated with achromatic-oriented and color-oriented stimuli in Area V2 neurons.

\section{Chapter 3: Clustered Cortical Organization and the Enhanced Probability of INTRA-AREAL FUNCTIONAL INTEGRATION...........................................................................................48}

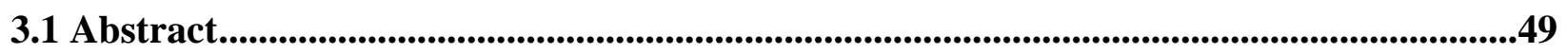

3.2 Introduction and Background .......................................................................................................50

3.3 Model Frameworks as Representations of Functional Domain Organization in Cortex54

3.4 Model 1 Methods: A Topographical Framework to Investigate Functional Integration Associated with Domain Pairs ....................................................................................................56

3.4.1 Two-Dimensional Representation of Neuronal Terminal Distributions ...................56

3.4.2 Two-Dimensional Representation of Functional Domain Organization ...................57

3.4.3 Mapping the Functional Joint Probability of Specific Color and Orientation

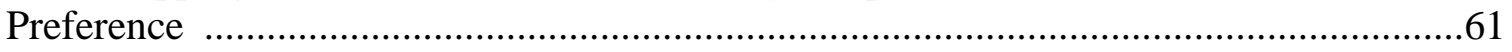

3.4.4 Controlled Manipulations of Model Domain Configurations and Associated Parameters.

3.4.5 Evaluation of Joint Probabilities of Co-Occurrence of Model Terminals and Model

Spines

3.4.6 Control Study: Variation of Domain Purity, Terminal Density and Bias Parameters

3.4.7 Control Study: Diffuse vs. Patchy Projection Distributions.

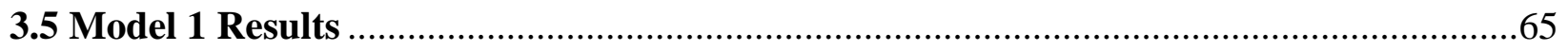

3.5.1 Configuration A: Random (No Domain) Organization .............................................65

3.5.2 Configuration B: Single Domain Organization .......................................................66

3.5.3 Configuration C: Paired Domain Organization ..........................................................67

3.5.4 Configuration D: Elongated Domain Shape ..........................................................69

3.5.5 Joint Probability of Model Terminal and Spines in Paired Domain Configurations 70

3.5.6 Functional Joint Probabilities Remain Enhanced Despite Primary Parameter

Manipulations .......................................................................................................

3.5.7 Functional Joint Probabilities Remain Enhanced Despite an Increase in Terminal

Patchy Redistribution.......................................................................................................74

3.6 Model 2: A Topographic Model Framework Incorporating Multiple Domain Proximity 


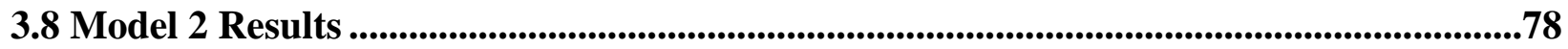

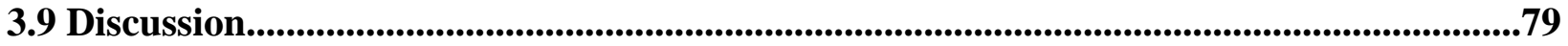

3.9.1 Mechanisms of Integration in V2: Inter-Areal vs. Intra-Areal ..............................80

3.9.2 An Explicit Role for Clustered Functional Organization? ......................................82

3.9.3 Permissive Advantages of Domain Organization and Larger Scale Stripe Geometry

in $\mathrm{V} 2$.

3.9.4 Choice of Parameters and Parameter Control Studies ....

3.9.5 Domain Organization and Intra-Areal Integration of Function as a General Principle

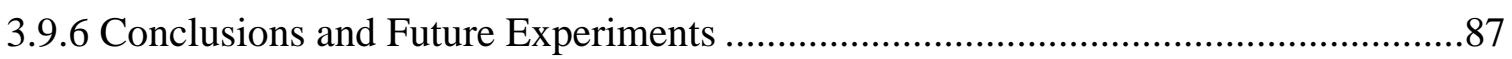

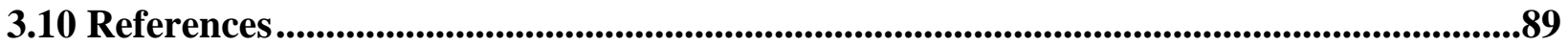

CHAPTER 4: SENSORy InTEgration ANd Temporal Response Modulation in MACAQue AREA V2: SINGLE UNIT ELECTROPHYSIOLOGY.............................................................................95

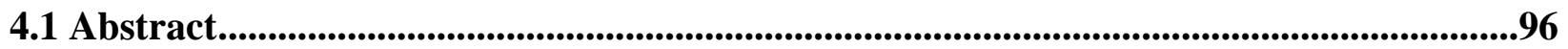

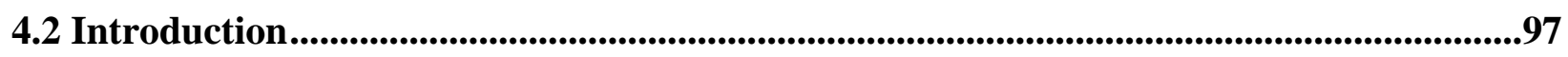

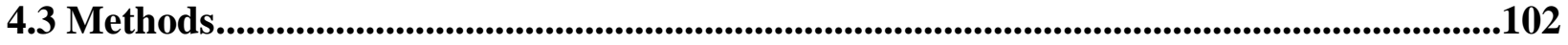

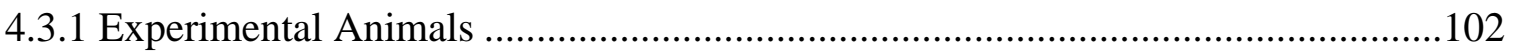

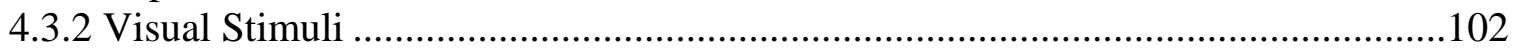

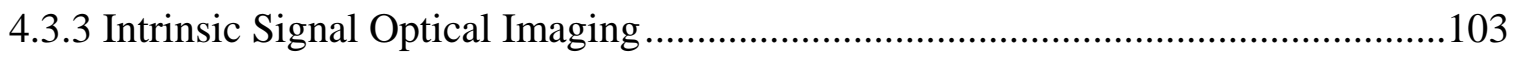

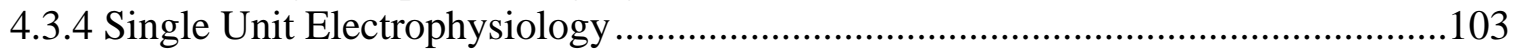

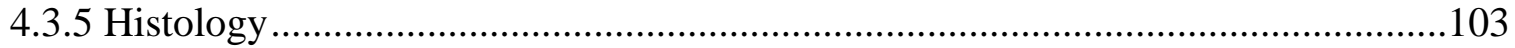

4.3.6 Preference and Latency Determination.........................................................103

4.3.7 Temporal Response Characteristics ...........................................................107

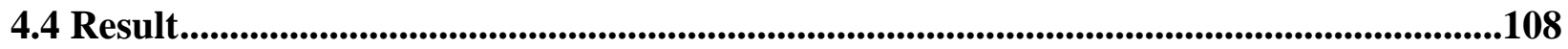

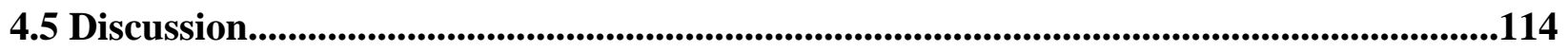

4.6 References ................................................................................................................................119

CHAPTER 5: MATERIALS, METHODS AND TECHNIQUES ..................................................124

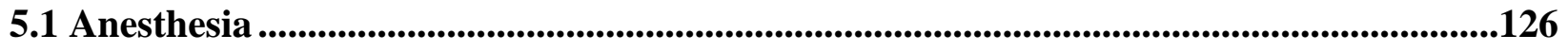

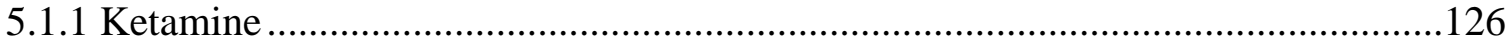

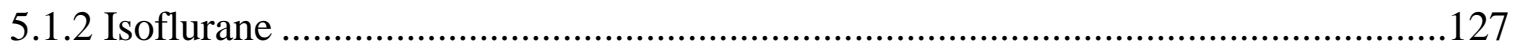




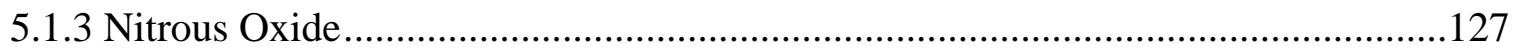

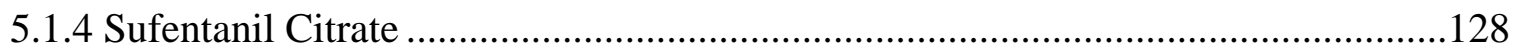

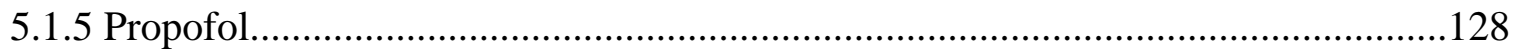

5.1.6 Vecuronium Bromide........................................................................................129

5.2 Surgical Procedure...............................................................................................................................130

5.2.1 Craniotomy and Durotomy ………………………................................................130

5.2.2 Artificial Dura Hat and Chamber Implant ........................................................130

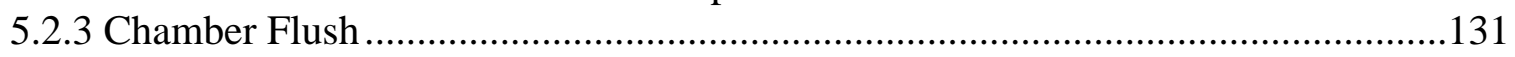

5.3 Intrinsic Signal Optical Imaging .............................................................................................133

5.3.1 Methodological Background.............................................................................133

5.3.2 Experimental Procedure.............................................................................................133

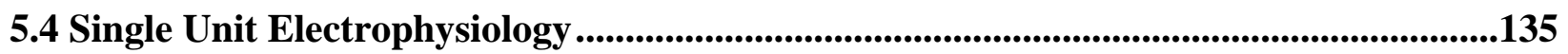

5.5 Experimental Stimuli...............................................................................................................136

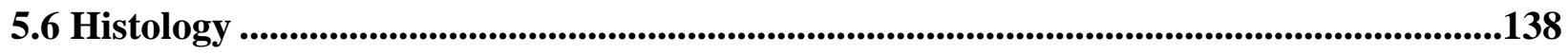

5.6.1 Vascular Perfusion............................................................................................138

5.6.2 Cytochrome Oxidase....................................................................................138

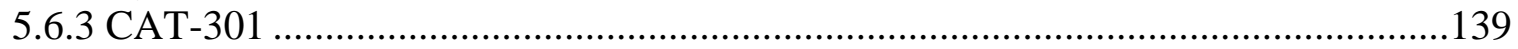

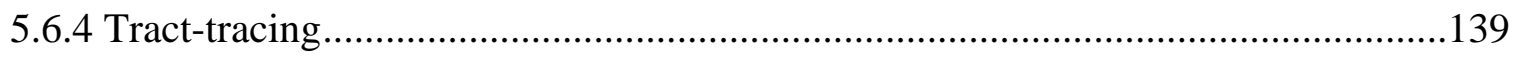

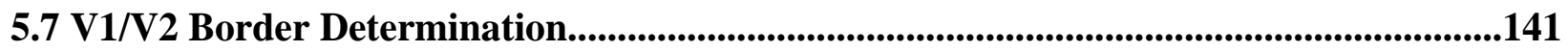

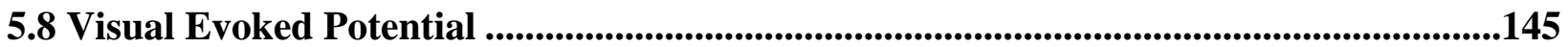

5.8.1 Materials and Methods........................................................................................146

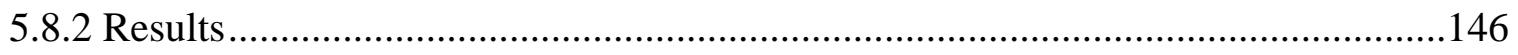

5.9 Baseline and Latency Calculations................................................................................................148

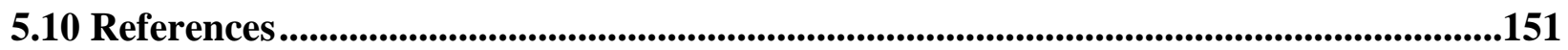

CHAPTER 6: GENERAL DISCUSSION ..........................................................................................154

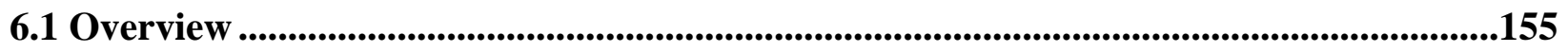

6.1.1 Computational Network Modeling …………………...............................................156

6.1.2 Characterization of V2 Neurons' Temporal Responses to Achromatic- and Color-

Oriented Stimuli Results ..............................................................................................158

6.2 Significance of the Results..............................................................................................................161

6.2.1 The Role of Clustered Organization, Intra-Areal Connections, and Mixed

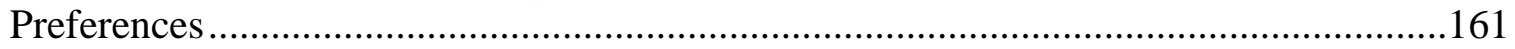

6.2.2 Limitations of Modeling Experimental Design and Interpretation..........................163 
6.2.3 Limitations of Electrophysiological Experimental Design and Interpretation .......164

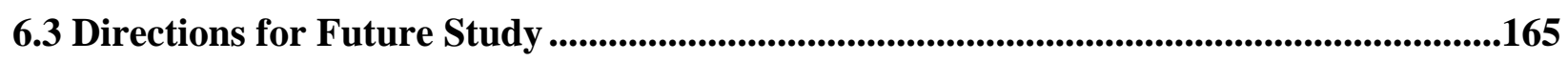

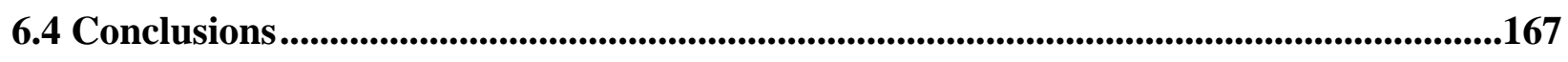

6.5 References .................................................................................................................................168

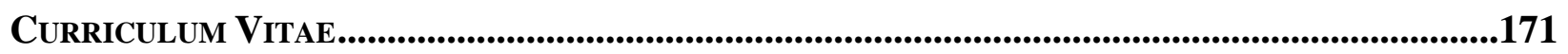




\section{List of Figures}

\section{CHAPTER 1}

FigURE 1-1. ANATOMY Of THE Primate Eye ..........................................................................

Figure 1-2. PhotoreCePtors TranduCE Light at the Retina........................................................5

Figure 1-3. Visual Pathways from the Eye to the Primary Visual Cortex .................................

Figure 1-4. Cytoarchitecture of Macaque Striate Cortex Processed with Cytochrome

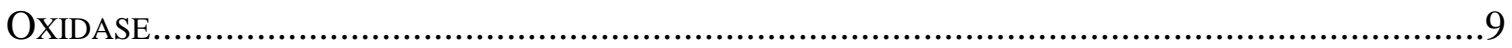

Figure 1-5. Comparison of Primary and SeCondary Visual CoRTEX ORganization .................12

Figure 1-6. Stripe Pattern of Macaque Secondary Visual CorteX Revealed With

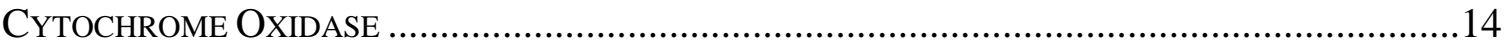

Figure 1-7. Putative Circuits Underlying Integrative Computation in Primate Area V2

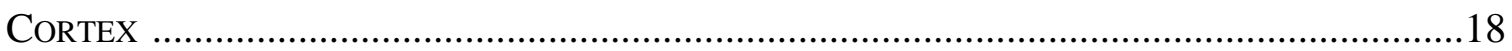

Figure 1-8. ObSerVed Latencies Of THE Primate Visual SYSTEM................................................22

Figure 1-9. EXAMPLE Of CluSters In THE SECONDARY Visual CoRTEX ...........................................31

\section{CHAPTER 3}

Figure 3-1. Clustered Cortical Organization: An Efficient Mechanism for Potent IntraAreal Integration of Neuronal InPUtS With Different Stimulus Preferences?.........51

Figure 3-2. Model Overview and Joint Probabilities in Random (No Domain) Preference

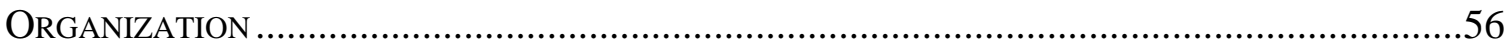

Figure 3-3. Domain Organization Significantly InCREASEs Poc Beyond the Defined Domain

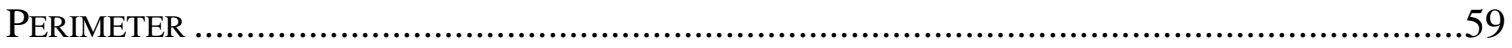

Figure 3-4. Paired Domain Configurations Yield EnHANCEd Poc Compared with Single DOMAIN OR RANDOM CONFIGURATIONS .............................................................................60

Figure 3-5. Cluster Elongation Further Elevates PoC.........................................................63

Figure 3-6. Color-Coded Poc Examples for Random, Single Domain and Paired Domain CONFIGURATIONS

Figure 3-7. Domain Proximity Increased Joint Probability of Color-PreferRing Terminals AND ORIENTATION-PREFERRING SPINES ...........................................................................71

Figure 3-8. Significant Enhancement in Joint Probability is Maintained Despite Substantial Variations in Domain Purity, Terminal Density and Bias ........................73

Figure 3-9. Elevated Joint Probabilities Persist with Patchy (CF. Diffuse) Terminal

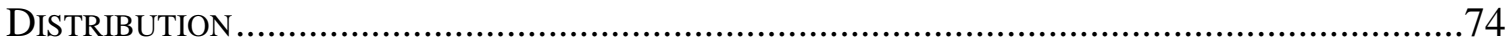

Figure 3-10. LARGE-SCALE PACKING CONSTRAINTS SUPPORT COMPREHENSIVE INTRA-AREAL

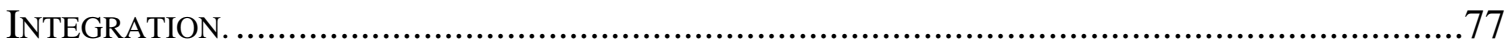

\section{CHAPTER 4}

Figure 4-1. InTRINSiC Signal Optical IMAged Difference MaP...................................................99 Figure 4-2. Examplar Cell for Preference Determination and Temporal Characterization

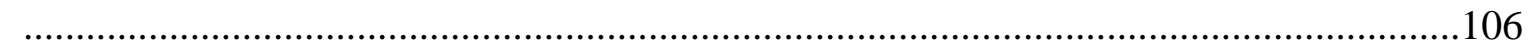

FiguRE 4-3. DistRIBUtion OF LATENCIES AND MAXIMUM FiRING TiMES .........................................109

Figure 4-4. Latency Modulation Due to Addition of Color to Orientation Bar.................110 Figure 4-5. Addition of Color to an Orientation Bar Lengthens Time to Peak Firing Rate 
Figure 4-6. Orientation and Color Preferences Do Not Affect Latency or Maximum Firing

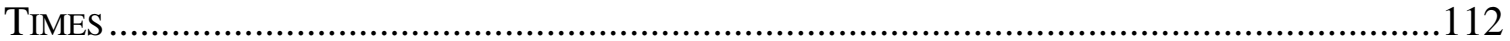

Figure 4-7. Chromatic Bar Maximum Firing Times and Maximum Spiking Activity Show

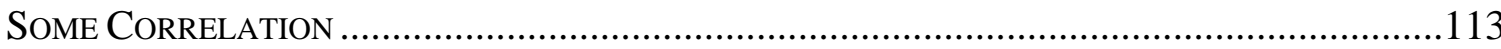

Figure 4-8. ChANGES IN LATENCY WiTH AdDITION OF COLOR TO AN ORIENTATION BAR IS CORRElated to THE CHANGES IN MAXIMUM Firing Time

Figure 4-9. Averaged Temporal Response Profile of Cells to Preferred-Color, PreferredOrientation Stimuli Display Reduced Spiking Response During the Initial Phase of Activity Compared to Preferred-Color, Prefered-Orientation Stimuli ..................115

\section{CHAPTER 5}

Figure 5-1. Transcranial Chamber and Artificial Dura Allow for Multiple Experimental PROCEDURES

Figure 5-2. V1/V2 BORDER CAN BE DETERMINED BY OPTICAL IMAGING OF OCULAR DOMINANCE AND ORIENTATION PREFERENCES.

Figure 5-3. V1/V2 Border CAn Be Determined by Histology and Penetrations can be MAPPED TO EITHER V1 OR V2

Figure 5-4. V1/V2 Border CAN Be Determined by Receptive Field Progression. .144

Figure 5-5. Visual Evoked Potentials Used to Determine Anesthetic Depth and Visual ACUITY 


\section{List of Abbreviations}

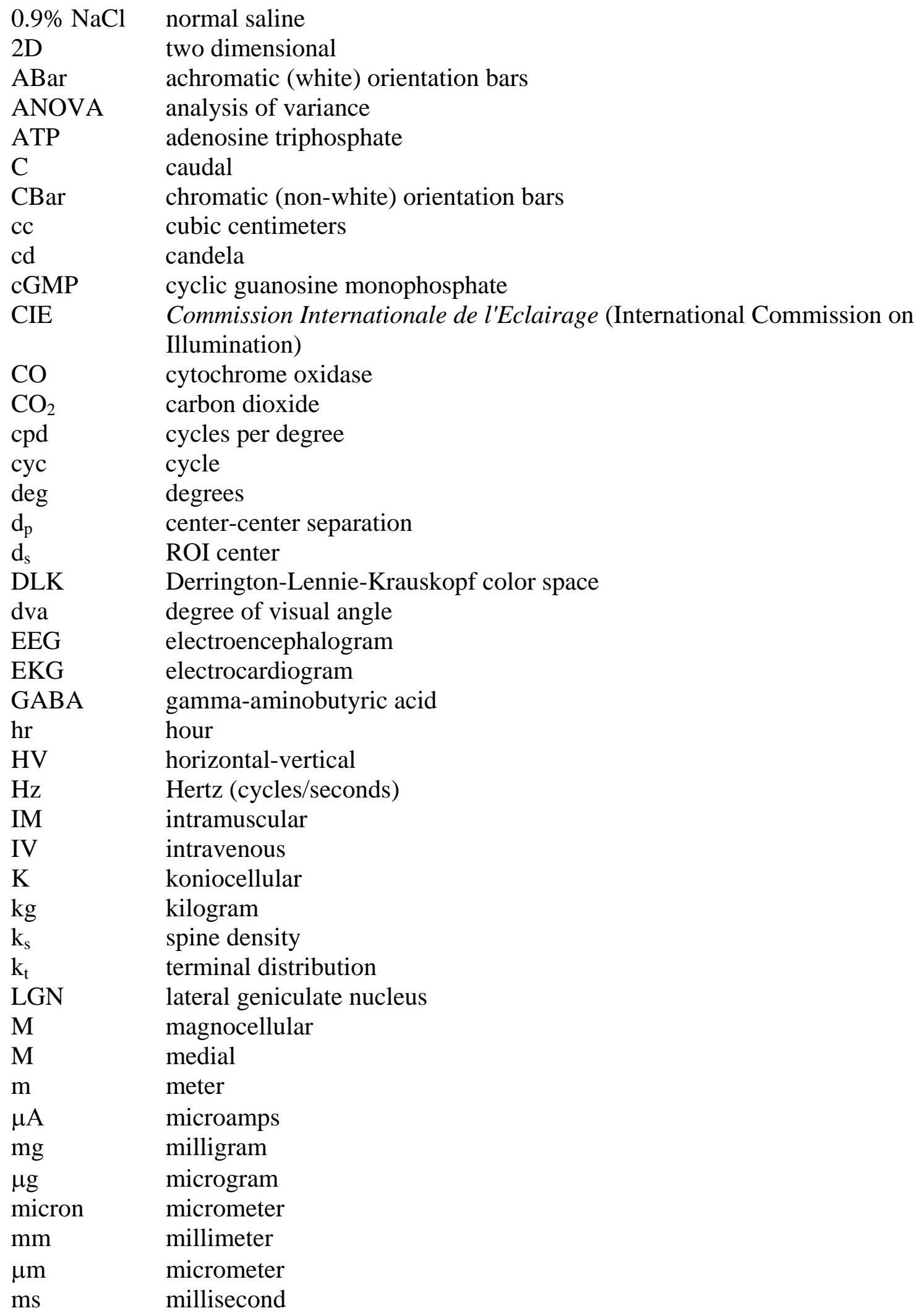




$\begin{array}{ll}\text { msec } & \text { millisecond } \\ \text { MT } & \text { middle temporal visual area (synonymous with V5) } \\ \text { MTF } & \text { maximum time until firing } \\ \text { nm } & \text { nanometer } \\ \text { NMDA } & \text { N-methyl-D-aspartic acid } \\ \mathrm{N}_{2} \mathrm{O} & \text { nitrous oxide } \\ \mathrm{O}_{2} & \text { oxygen } \\ \text { OB } & \text { acute/oblique } \\ \text { p } & \text { p-value } \\ \text { P } & \text { parvocellular } \\ \mathrm{P}_{\text {OC }} & \text { functional joint probability } \\ \mathrm{P}_{\text {OC-ROI }} & \text { functional joint probability of the region-of-interest } \\ \mathrm{P}_{\mathrm{OC} 9} & \text { functional joint probability at the } 99^{\text {th }} \text { percentile } \\ \mathrm{PET} & \text { positron emission tomography } \\ \mathrm{r} & \text { Pearson product-moment correlation coefficient } \\ \text { RF } & \text { receptive field } \\ \text { ROI } & \text { region-of-interest } \\ \mathrm{r}_{\text {prox }} & \text { proximity hit rate } \\ \text { RT } & \text { reaction time } \\ \text { S } & \text { seconds } \\ \text { SD } & \text { standard deviation } \\ \text { SDF } & \text { spike density function } \\ \text { sec } & \text { second } \\ \text { V1 } & \text { primary/striate visual area } \\ \text { V2 } & \text { secondary visual area } \\ \text { V3 } & \text { third visual area complex } \\ \text { V4 } & \text { fourth visual area } \\ \text { V5 } & \text { fifth visual area (synonymous with MT) } \\ \text { VEP } & \text { visual evoked potential } \\ \text { VM } & \text { vertical meridian } \\ \text { WVU } & \text { West Virginia University } \\ & \end{array}$


CHAPTER 1: Introduction and Literature Review 


\subsection{Overview of the Visual System: Retina to V1}

Every day we automatically and without conscious effort interpret many different aspects of a visual stimulus. An object's shape, luminance, lines, and edges are all seamlessly integrated into a complete image perceived by the visual cortex. We 'see' these components not as parts that one must then piece together, but as complete and whole within the visual field. These images begin as individual chemical reactions within the retina and are transduced into electrical signals, or neural spiking activities, that are then processed in turn by multiple cortical areas that are devoted to the interpretation of visual information and ultimately 'telling' the brain what it is viewing. The basic sense of vision, the most relied upon source of external information for primates, is certainly complex.

Psychophysical studies have indicated the presence of the "horizontal effect" in human vision - subjects' performance for detecting oriented content was optimal with oblique angles, intermediate with vertical orientations, and worst at horizontal (Essock et al., 2003). This effect is most noticeable in natural scenes, suggesting that orientation encoding evolved to downplay the prominent orientation (the horizon), increasing the resulting response to unusual orientations (oblique and vertical angles) against a natural background (Hansen and Essock, 2004). Nonhuman primates, like us, also commonly use color to aid in the rapid identification of visual forms, which adds additional information beyond luminance contrasts thereby providing significant cues for detecting elements in a visual scene. The evolutionary advantage of selective detection of ripened, colorful fruit amongst numerous green leaves has been theorized to be a catalyst for the development of primate color vision (Allman, 1999). Experimental recordings from early non-human primate visual cortical areas indicate that some single neurons show elevated activities in response to both orientated and colored stimulus attributes, suggesting the importance of the integration of these visual stimulus components. The mechanisms of neuronal integration that underlie combined color and orientation processing are still poorly understood. Many neurons in primary visual cortex (Area V1) are reported to preferentially respond to elementary color or orientation stimulus attributes and these activities are known to be routed onto further 'higher' visual cortical areas (Areas V2, V3, V4, etc.) where neurons respond to more complex combinations of stimulus attributes. The convergence of neurons from lower to higher areas in visual cortex has long been described as mechanism by which neurons may form the basis for key signaling of complex visual features such as the rim of a cup, the corner of a 
box, or even selectivity for a specific human face (Day and Kasperczyk, 1983; Kanwisher et al., 1997). The second visual area (V2) receives predominant neuronal input from V1, and exhibits many neurons with mixed color and orientation properties. This dissertation seeks to afford a better understanding of the neural circuitry and mechanisms underlying the processing of color and orientation information in Area V2 primate visual cortex of the cynomolgus macaque (Macaca fascicularis).

The macaque's visual processing system (retinal and visual cortical structures) is strikingly similar to that of the human and it is known that macaques experience many of the same visual perceptions of color and orientation as humans (Maguire et al., 1980), making it an excellent animal model (Hanazawa et al., 2001). By improving our understanding of these processes in the macaque monkey, we may learn more about the mechanisms associated with basic human vision. In addition to animal models, computer modeling has also proven to be a useful tool to better understand visual function (Szeliski, 1990). Computer models can be especially valuable because they allow the development and testing of large but well-controlled artificial networks of neuron-like elements. These biologically inspired networks can be systematically manipulated (functional parameters and structural connectivity organizations) in ways not possible in current animal models, and thus computational experiments can be used to learn how complex neuronal responses might arise from particular network organizations.

It is known that neurons in early visual areas such as V1 and V2 are organized into clustered groupings ("functional domains") wherein shared preference for a particular color or orientation is highly probable. One possibility is that these clustered neural organizations provide some anatomical advantage for the pooling of outputs from neurons of different functional preference - thus providing efficient input circuitries for neurons that in turn prefer a particular color/orientation combination. The work here intends (i) to investigate the potential role of clustered organization (functional domains) in the integrated neuronal response to both color and orientation using computer modeling methods and (ii) to further characterize some aspects of the generation of mixed properties in V2 using in vivo experimental methods using the macaque animal model. In particular, the modeling and experimental recording studies presented in this dissertation characterize and investigate how color and orientation is processed together in primate Area V2. 
The remainder of this chapter will summarize relevant anatomical and functional background associated with color and orientation processing from retina to the lateral geniculate nucleus (LGN) (Section 1.1.1), V1 (Section 1.1.2) and V2 (Section 1.2). This is followed by a review of the principles of clustered organizations (Section 1.3), mechanisms involving latency of spiking V2 neurons (Section 1.4), the established methods for using specific visual stimuli to probe the function of early visual cortices (Section 1.5) and finally the sensory processing models that may help elucidate more complex principles of visual processing (Section 1.6). The results of computer models of functional domain interaction in V2 (Chapter 2) and the characterization of temporal modulations in V2 associated with the introduction of color to an oriented stimulus (Chapter 3) are discussed in the later chapters.

\subsubsection{Visual Processing and Neuronal Functional Organization: Retina to Lateral Geniculate Nucleus}

Before examining the interaction of color and orientation in the higher levels of visual processing, it is necessary to understand how this information reaches these extrastriate areas. The processing of color vision begins within the eye, a highly specialized receptor and direct extension of the central nervous system (Figure 1-1) (Pei and Rhodin, 1970; Barishak, 1992). Light waves emanating from an external source are directed through the cornea and lens of the eye to the posterior region of the eye cavity where the photoreceptors are located. The photoreceptors generate neural responses depending on the intensity and wavelength characteristics of the incoming light. The two types of photoreceptors, rods and cones, are positioned along the outside of the retina (O'Brien, 1982): rods are primarily active at low levels of luminance while cones are

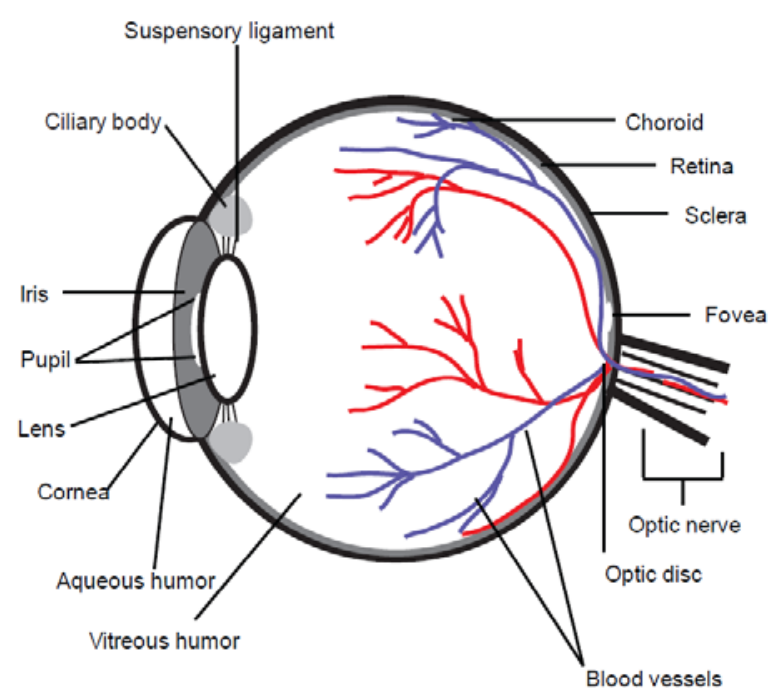

Figure 1-1. Anatomy of the primate eye. Refracted by the cornea and the lens, incoming waves of light are focused towards the fovea located at the posterior of the eye. Photoreceptors are located here along the retina and activated by the arriving light. Cones, receptors stimulated by color wavelengths, are prominent in the fovea and luminance sensitive rods are more prevalent in the periphery. After the receptors respond to the light, visual information is transmitted out of the eye along the optic nerve to visual areas of the brain. 
more likely activated at higher luminance levels (Curcio et al., 1987). The cones are located immediately next to the pigment epithelium layer of the retina and are most prevalent at the fovea (Curcio et al., 1990). Images of interest, or "focus" in the visual field, are projected to this fovea, which is positioned at the epicenter of the back of the eye (central $2^{\circ}$ ) (Borwein, 1983). There are three major categories of cones documented: the S-, M-, and L-cones. Each of these cone types reacts more strongly to limited but different ranges of the visual spectrum: S-cones react most intensely to shorter wavelengths (peak response $\sim 450 \mathrm{~nm}$ ) and thus respond best to 'blue' light, M-cones and L-cones react to longer wavelengths (M-cones peak response 525 nm, L-cones peak response $\sim 555 \mathrm{~nm}$ ). Thus M-cones show stronger response to green rather than red light and L-cones show stronger response to red rather than green light. Rods, conversely, possess only a single type of photopigment and are sensitive to a wide spectrum of wavelengths but have a higher efficiency of light absorption, allowing activation from lower levels of light. The presence of these three cones and their combined outputs are thought to allow the precise discrimination of a very broad range of light wavelengths in normal 'trichromatic' vision. Importantly, this retina type is observed in Old World primates such as the macaque genus as well as humans (Brown and Wald, 1964; Marks et al., 1964), but is relatively uncommon in most other vertebrates where dichromacy (2 cone types) is more prevalent.

At the retina, light is converted into an neural signal and transmitted to the lateral geniculate nucleus of the thalamus (LGN), and eventually the cortical areas V1 and V2 (Figure 1-2). When a light wave strikes a cone, the pigment molecules located in the outer segment of

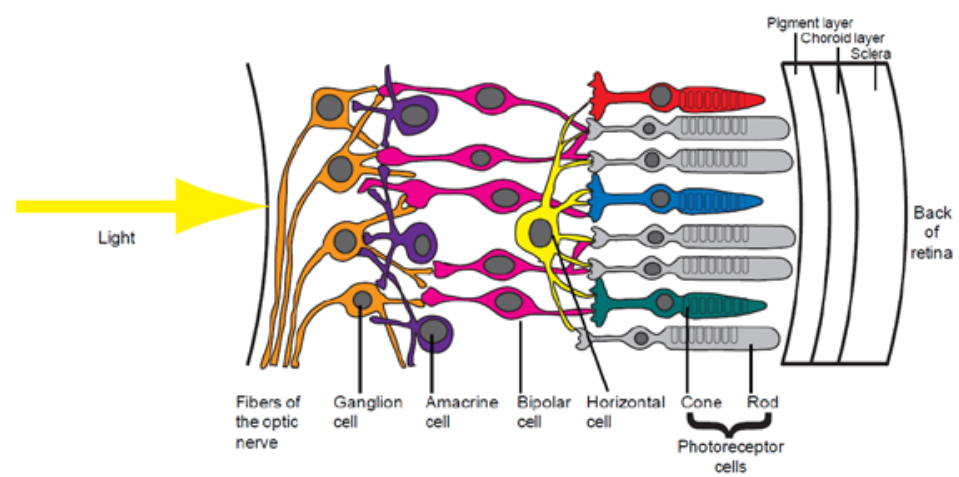

Figure 1-2. Photoreceptors transduces light at the retina. When light reaches the photoreceptors, the pigment molecules activate and ultimately hyperpolarize the cell. This potential change inhibits the bipolar cells, propagating the signal to the ganglion cells thorough the interneurons horizontal and amacrine cells. The visual information projects out of the ganglions along the fibers of the optic nerve. the photoreceptors absorb the light and undergo a conformational change. This activates the G-protein transducin, which then stimulates cGMP phosphodiesterase, leading to a reduction in cGMP in the cytoplasm and, as a result of this decrease, closes the cGMP-gated cation channels. The closure of these channels reduces the current and leads to hyperpolarization at the distal portion of the receptor (Miki et al., 1973; Hurwitz et al., 1985). This 
potential change closes $\mathrm{Ca}^{++}$channels at the synaptic terminal of the cone, leading to lightdependent reduction in neurotransmitter glutamate release; this modulation yields an inhibition of "Off" bipolar cells, excitation of "On" bipolar cells and graded changes in potential. The changes of numerous bipolar cells are pooled and transferred to ganglion cells and this may involve the engagement of interneurons known as amacrine and horizontal cells (Yamada and Ishikawa, 1965; Dowling and Boycott, 1966; Masland, 1986). Ultimately, these projections impact on the retinal ganglion cells (action-potential producing neurons that are the primary neural exit from the retina). Thus ganglion cells respond selectively and differentially to the diverse wavelengths of light incident on the retina as a result of various inputs from multiple (types) of cones. The subsequent inputs onto ganglion cells create an approximately circular receptive field in visual space for each retinal ganglion cell. Visual space is the visual perception of space, or rather how visual stimuli are interpreted by the visual system. A receptive field (RF) is the area of the retina that a ganglion cell monitors; the cones and rods of an area of the retina are typically directed to the same ganglion cell(s) leading to a summation of photoreceptor activity from that particular region. The ganglion cell RF is divided into the center and surround; the center refers to the circular center area of the RF and the surround is the remaining area on the edges of the field. The differential illuminations of the center and surround thus provide spatially antagonistic inputs to increase or decrease activity in the ganglion cells. Visual stimulation of ganglion cell RFs typically evokes increases in neural spiking when stimulation includes local luminance contrasts (e.g., bright center vs. dark surround), or stimulus color contrast (e.g., a red center vs. green surround). The different response to color vs. luminance contrast have been linked to the so-called parasol (large dendrite arbor) and midget (small dendrite arbor) ganglion cells, two important types of ganglion cells. Axons from these retinal ganglion cells project out of the eye at the point of the optic disc, a region lacking any photoreceptors, via the optic nerve and terminate on the LGN (de Monasterio, 1978a, b). Importantly, these two classes of retinal ganglion cells are known to project to distinct layers of the LGN, and their selective responses to different spatiotemporal aspects of the visual stimulus thus project different electrophysiological profiles to their distinct layered terminations in the LGN. The parasol cells project to the 'magnocellular' layers of LGN, and the midget cells project to the 'parvocellular' layers of LGN, circuitries that are also commonly referred to both anatomically and functionally as the magnocellular (or ' $\mathrm{M}$ ') and parvocellular (or 'P') pathways 
(or 'channels') respectively. The parasol ganglion cells (and their terminal recipient magnocellular layer LGN cells) tend to evoke higher firing rates to visual stimuli containing luminance contrast and high temporal/low spatial frequencies, whereas the midget ganglion cells (and their terminal recipient LGN parvocellular layer cells tend to prefer high spatial/low temporal frequencies and lack luminance sensitivity but do possess greater wavelength specificity (Shapley and Lennie, 1985).

In the retina, adjacent neurons have overlapping receptive fields that detect distinct portions of the visual field. This 'retinotopic' spatial organization remains prevalent throughout many stages of the visual system, including the LGN (Erwin et al., 1999) and the striate and

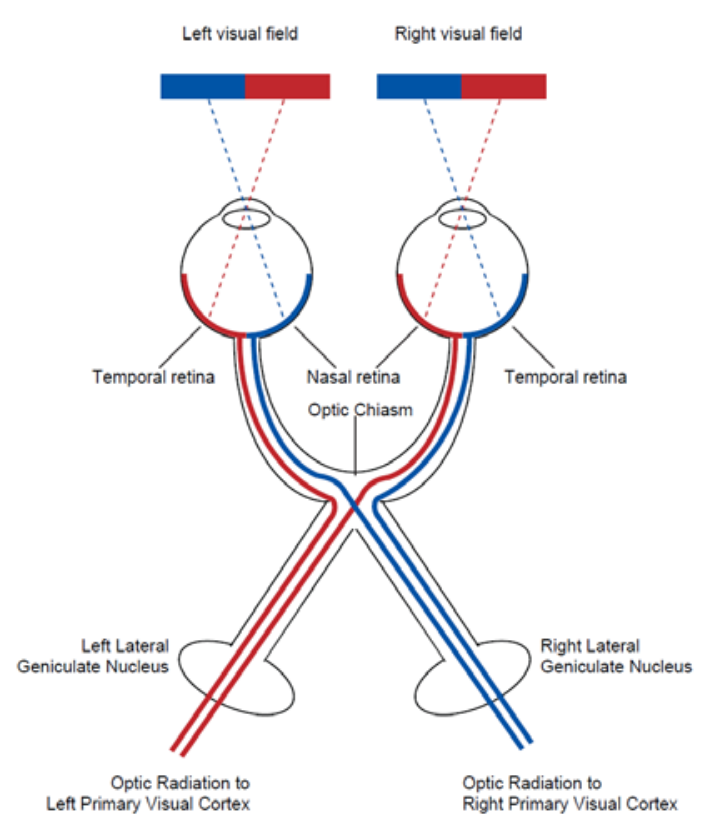

Figure 1-3. Visual pathways from the eye to the primary visual cortices. Visual field information is projected from the retinas, crosses the optic chiasm to the lateral geniculate nucleus (LGN) then on to the primary visual cortex (V1). The left visual field (blue) is projected to the right LGN and V1 and the right visual field to the left LGN and V1. Information from both fields is integrated at V1.fibers of the optic nerve. many extrastriate visual cortices (such as V2) (Rosa, 2002). As a result of this retinotopy, visual information arrives at the LGN based upon specific visual field activation (Figure 1-3). Each retina is divided into left and right hemifields; the left hemifield of each eye receives information from the right visual field and vice versa. This information remains organized within the eye until the optic chiasm, at which point the right hemifield information (containing visual images of the left field; right temporal hemiretina and left nasal hemiretina) projects to the right LGN while the left LGN receives projections from the left hemifield (left temporal hemiretina and right nasal hemiretina) of each eye (Stone et al., 1973; Chalupa and Lia, 1991). These bipartite projections continue to the striate and extrastriate cortices of each respective occipital lobe.

The retinal projections onto the LGN are organized in distinct layers. The midget ganglion cells (thought to transfer information about the differential balance of L vs. M cone input at the retina) terminate on the four small cell "parvocellular” layers of the dorsal LGN. The parasol ganglion cells (thought to transfer information about integrative sum of $\mathrm{L}+\mathrm{M}$ cone 
inputs) project to the two larger cell "magnocellular" layers of ventral LGN (De Valois et al., 1958). The inputs from LGN laminae 1, 4, and 6 arrive from contralateral nasal retina; the ipsilateral temporal hemiretina sends afferents to layers 2, 3, and 5 (Wiesel and Hubel, 1966). More recently, a third channel of vision processing, appears to involve bistratified retinal ganglion cells (thought to transfer information predominantly about S-cone activation preference) that project to dorsal, medial and ventral "koniocellular" layers between the $\mathrm{M}$ and $\mathrm{P}$ layers of the LGN. Dorsal ' $\mathrm{K}$ ' cells project low acuity information to layer 1 of the primary visual cortex (V1) and ventral K cells interact with the superior colliculus. The middle K cells are of particular interest, as they seemingly relay S-cone inputs directly to cytochrome oxidase (CO) blobs in V1. The presence of K pathways suggests visual processing may occur through more than two relatively discrete pathways (i.e. M and P pathways only) (Hendry and Reid, 2000; Casagrande et al., 2002) from retina to LGN, each favoring different cone input activation combinations.

\subsubsection{Visual Processing and Neuronal Functional Organization: Primary Visual Cortex}

In human cortex, V1 (also termed the primary visual cortex or the striate cortex) corresponds with Brodmann's Area 17. This area lies below the cuneus and immediately surrounds the calcarine sulcus on the medial portion of each occipital pole; Area 17 curves outward and laterally, encompassing the apex of the occipital lobe (the most caudal point of the forebrain) (Brodmann, 1905). In the macaque brain, the visual cortex in toto comprises over $50 \%$ of the cerebral cortex. V1 spans $\sim 1120 \mathrm{~m}^{2}$, making it the second largest cortical area (V2 is slightly larger). The lunate sulcus of the macaque brain lies on the superior surface of the cortex, approximately 15-20 $\mathrm{mm}$ from the most caudal edge, and immediately rostral to this sulcus lies a portion of V2, proceeded by the entirety of V1 (2 - 5mm from the lunate sulcus) (Felleman and Van Essen, 1991). This location is particularly amenable for study using electrophysiology techniques, as will be detailed in Chapter 4 and Section 5.3, respectively.

While LGN cells exhibit mostly circular receptive fields and therefore mostly have very poor orientation selectivity, many cells in V1 and V2 do show pronounced orientation selectivity (elongated receptive fields that evoke elevated spike firing to presented visual stimulus bars or edges at the 'preferred' orientation) when tested electrophysiologically. In V1, orientation

selectivity is thought to arise largely from specific convergence of inputs from LGN onto 
'simple' cells of V1 (Hubel and Wiesel, 1974), or subsequent inputs from simple cells onto oriented 'complex' cells of V1. This principle of generating new stimulus feature selectivity by virtue of convergence (i.e. integrated or summed inputs) between LGN and V1 or between other "lower" and "higher" cortical areas in the cortical organizational hierarchy (e.g. V1 onto V2) (see Felleman and Van Essen review) may be a fundamental principle of sensory processing in the brain.

Primate V1 possesses a distinctive columnar cellular organization based upon a cell's receptive field preferences: cells that respond selectively to the same stimulus attributes tend to be located in similar sub-millimeter sized clusters across the cortical area that span many or all of the cortical layers. At least two distinct but spatially overlapping columnar systems of cells exist in V1: one arrangement of columns contains cells with orientation receptive field's preferences and another system is organized by ocular dominance (i.e. receiving visual input dominated by one eye) (Hubel and Wiesel, 1969). A complete set of preference columns are repeated across the cortex, forming hypercolumns (a set of columns containing all preference types in a region of cortical space) (Hubel and Wiesel, 1977). In the lower regions of layers 3, 4A, and 4B: many cells may be classified as "simple" and roughly half are dominated by one eye with the remainder dominated by the other eye. In other layers cells are predominantly "complex" but with binocular preference (although usually one eye has net preference over the other) (Hubel and Wiesel, 1968).

Further V1 architecture can be observed through the use of cytochrome oxidase (CO), an endogenous metabolic marker associated with oxidative metabolism and ATP synthesis (Figure 1-4) (Wong-Riley, 1989). The CO enzyme, located

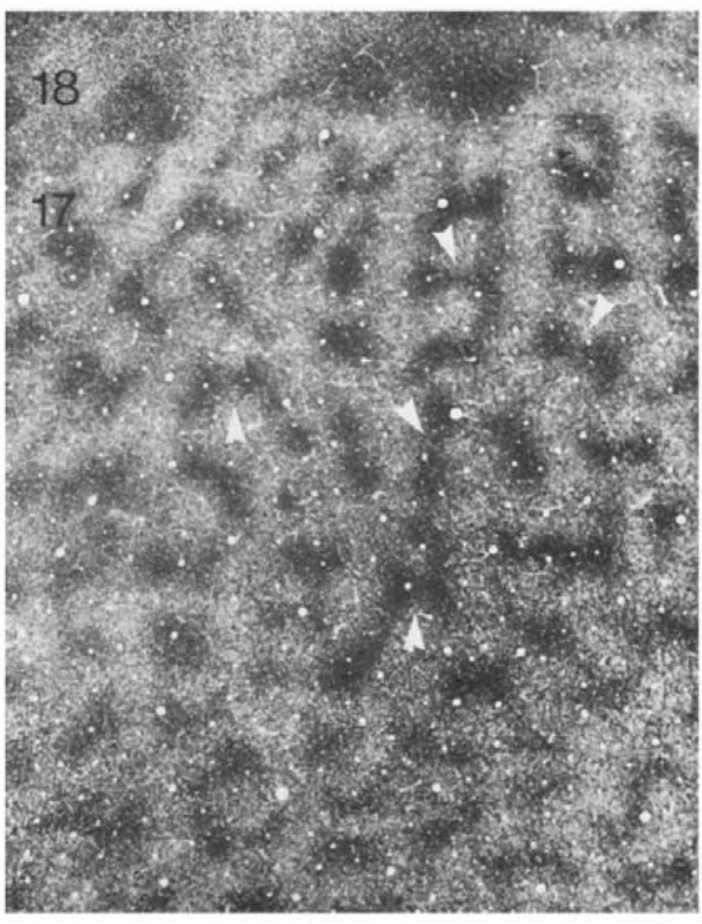

Figure 1-4. Cytoarchitecture of macaque striate cortex processed with cytochrome oxidase. Cytochrome oxidase (CO) stained section of monkey striate cortex, including the area 17/18 (primary/secondary visual cortex) border. Darkstaining areas define blobs (containing color preferring cells) and the paler areas are defined as the inter-blob regions (populated with orientationpreference cells). Arrows indicate the bands of staining between individual blobs. This figure is adapted from another source (Ts'o and Gilbert, 1988). 
within the mitochondria of neurons and neuropil, is highly associated with functioning vision. When tangential sections of V1 are histologically processed with CO, patchwork oval regions of

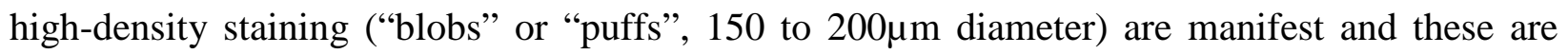
typically centered along the ocular dominance columns of V1. Spaced $\sim 350 \mu \mathrm{m}$ apart, the blobs align along the wider axes in parallel linear arrangements that are perpendicular to the V1/V2 border (Wong-Riley, 1979). These histological markers are used to delineate the so-called 'blob' and 'inter-blob’ areas within and across V1.

Previous electrophysiological investigations have indicated that the interblob region of V1 is highly populated with orientation preferring cells, however orientation selectivity of neurons located increasingly closer to the blob regions dropped off drastically - cells displayed no orientation preference within the area horizontally encompassed by the CO blob and vertically down to layer 4A (Livingstone and Hubel, 1984b). Additional electrophysiological studies, electrode tract reconstruction, CO histology, and electrolytic lesions confirmed that CO blobs contain unoriented cells, but many of these cells were color selective, displaying circular receptive fields similar to those observed in the LGN (Hubel and Wiesel, 1968; Livingstone and Hubel, 1984b, a; Ts'o and Gilbert, 1988). Red/green and yellow/blue blobs were reported at a ratio of 3:1 (Ts'o and Gilbert, 1988), similar to the proportion of opponency cells seen within the LGN (Hubel and Wiesel, 1969; Ts'o and Gilbert, 1988). Bridging of connections between blobs with similar color receptive fields across unlike ocular dominance columns has been observed (Ts'o and Gilbert, 1988). Injections of horseradish peroxidase into layers 2 and 3 of blob and interblob regions yielded similar results: the label was localized within a region and only transported to like regions (i.e. injections in a blob skipped neighboring interblob regions and only reappearing in surrounding blobs) (Livingstone and Hubel, 1984a). However, using Lucifer Yellow, the blob’s dendritic spread was found to be significantly larger than the spread from the interblob region and large enough to reach neighboring blobs (Elston and Rosa, 1998). Other studies of color preferring cells aligning with CO blobs further support the functional partition of color and orientation processing between blob and interblob regions of V1 (Dow and Vautin, 1987; Tootell et al., 1988). The theory of segregation of orientation and color processing became well-established from these reported findings, and with the publication of a landmark review by Livingstone and Hubel in Science (Livingstone and Hubel, 1988). These scientists concluded that cells tend to be either color or orientation selective in V1 and rarely selective for both color 
and orientation, and that these functional properties correlate well with blob and interblob compartments (Felleman and Van Essen, 1991; Roe and Ts'o, 1999; Sincich and Horton, 2005). Other studies observed and proposed a less distinctive view of V1 blob/interblob functional organization. Throughout layers 2/3 of V1, most cells contained a preference for orientation and color (and also other properties such as direction of motion) to varying degrees (Leventhal et al., 1995). Furthermore, V1 color cells were documented away from CO blobs and possessing more complicated color preferences than seen in LGN (Lennie et al., 1990). These discrepancies may perhaps be explained by different technical aspects of the experiments that constrain the precision of alignment of recording sites with CO compartments, or the inability to precisely delineate where compartments begin and end across V1 cortex. Additionally, color-oriented neurons have been reported in V1 (Roe and Ts'o, 1999; Johnson et al., 2001; Friedman et al., 2003); Landisman and Ts'o (2002a, b) suggesting that these may be more prevalent along the borders of the blob/interblob regions. In recent years, the functional organization for color and orientation selectivity in V1 cortex has been "mapped" using intrinsic optical imaging methods. This video imaging method detects very small changes in reflected visible light (typically red light, projected onto surgically exposed cortex) that occur in response to local hemodynamic changes. Difference mapping analysis can identify which parts of cortex (limited to the superficial three cortical layers) are better activated to one stimulus condition vs. other stimulus conditions. Individual difference maps can be further processed and combined to yield detailed high resolution overlays of color and orientation preference in V1. Orientation maps organize into "pinwheels" (named because of their appearance) with all orientations represented in progression (i.e. areas with a preference for $45^{\circ}$ is flanked by, for instance, areas preferring $30^{\circ}$ and $60^{\circ}$ ). Since the orientation map is mainly continuous, a pinwheel configuration allows for complete representation of all orientation preferences when parallel rows of preferences are absent (Blasdel, 1992a,b). Landisman and Ts'o (2002a,b) used intrinsic optical methods in conjunction with electrophysiological sampling to further support the general thesis of detectable segregation of some color vs. orientation preferring neurons in V1, with the possibility of mixed color/orientation processing at the blob/interblob borders (Figure 1-5). In summary, these recent studies suggest the possibility that many cells in V1 maintain predominantly distinct color and orientation processing. 


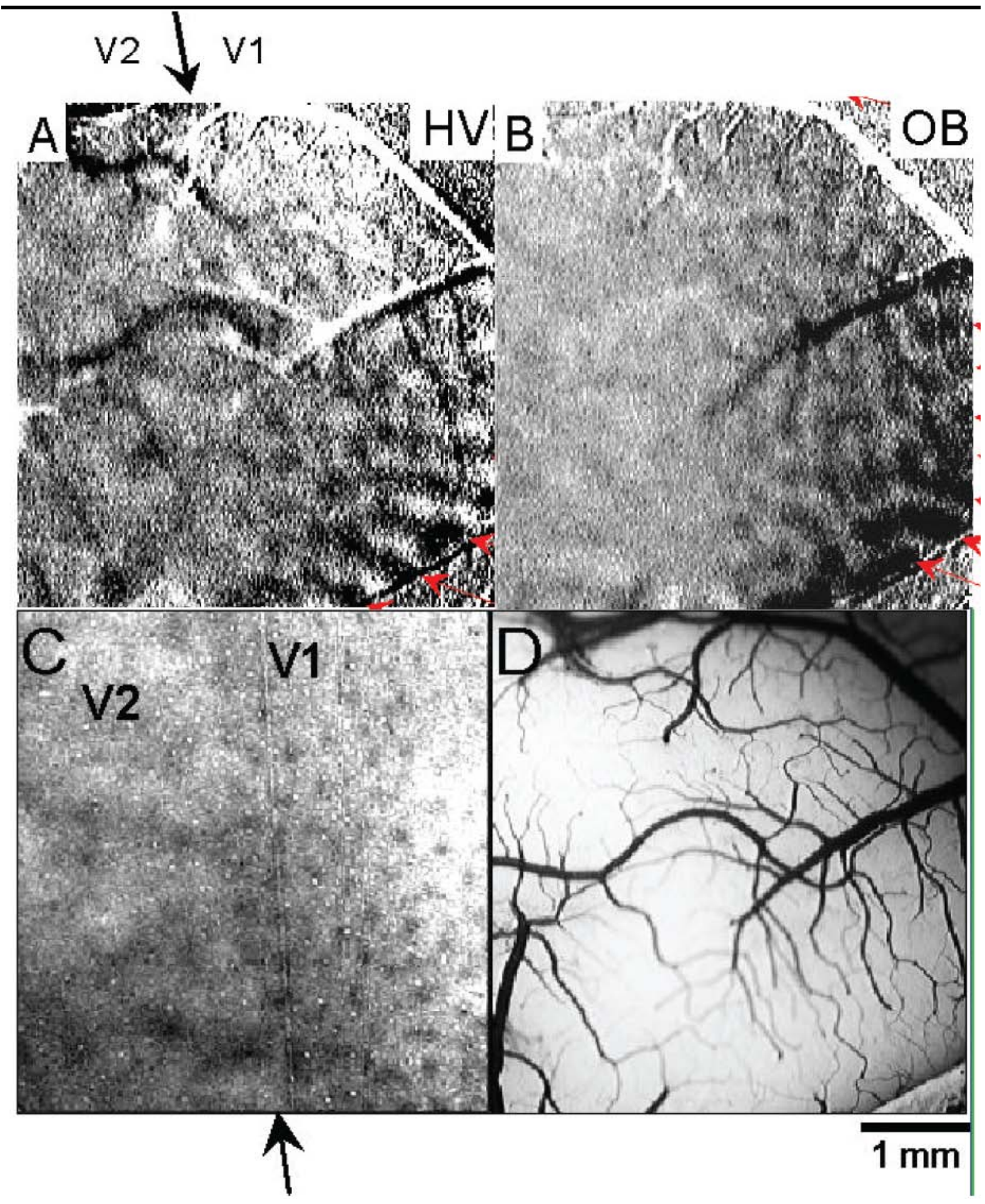

Figure 1-5. Comparison of primary and secondary visual cortex organization. Optical imagining $(A, B)$, cytochrome oxidase (CO) stained section $(C)$ and vessel map $(D)$ of monkey striate cortex, including the area V1/V2 (primary/secondary visual cortex) border. Difference maps of horizontal-vertical (HV) and acute/oblique (OB) highlight areas of the cortex with an orientation preference. Dark-staining areas define blobs (containing color preferring cells) and the paler areas are defined as the inter-blob regions (populated with orientation-preference cells). Note the overlap/correlation between the imaging and CO results. This figure is adapted from another source (Roe, 2005). 
Cells of the magnocellular, koniocellular and parvocellular layers of LGN project to different layers and/or CO compartments within V1 (Sawatari and Callaway, 1996). Parvocellular input from LGN (mediating L- and M- cone differential inputs enter $4 \mathrm{C} \beta$, while Sand $(\mathrm{L}+\mathrm{M}-)$ (carrying yellow color information) cones synapse onto layers $3 \mathrm{~B}$ and $4 \mathrm{~A}$ of the cortex comprising a V1 blob. An S-cone "On" afferent is located in layer 4A, an S-cone "Off" afferent is in $4 \mathrm{~A}$ and 2/3 while orientation information inputs into the corresponding interblob laminae (Schein and de Monasterio, 1987; Chatterjee and Callaway, 2003). However, other evidence illustrated that both blobs and interblobs receive input from magnocellular (M) and parvocellular (P) pathways (Yabuta and Callaway, 1998). The magnocellular pathway projects motion to layer 4B (Sincich et al., 2004). A comparison of the cellular densities of the dorsal LGN and V1 indicates that the number of $\mathrm{P}$ cells remains approximately constant with eccentricity whereas the number of M cells increases dramatically in V1. This indicates that the density of color cells from the LGN can estimate the degree of V1 magnification.

There have been differing experimental findings regarding the segregation of M- and Ppathway within the striate cortex, but there now appears to be a consensus for a substantial mixing of $\mathrm{M}$ and $\mathrm{P}$ pathways especially for circuits that favor processing of objects/form, rather than motion. From V1, these pathways extend through the prestriate cortex (or V2 extrastriate cortex (Gazzaniga et al., 2002)), the M-dominated stream projects into the posterior parietal cortex as the dorsal cortical pathway and the mixed M/P-stream continues into the inferior temporal cortex as the ventral cortical pathway (Livingstone and Hubel, 1987; Merigan and Maunsell, 1993). In other words, the ventral stream appears to play the major role in the identification of objects (i.e. form/color); the dorsal stream appears to mediate motion detection and identification of spatial location (Goodale and Milner, 1992). Positron emission tomography (PET) scans of the human brain indicate the separate responses to motion and color: moving stimuli led to an increase of activity in the areas surrounding V3 and the fusiform gyrus whereas more medioinferior areas of the occipital cortex displayed an increase in blood flow in response to color stimuli (Zeki et al., 1991). Both of these streams then pass through V2, with the dorsal pathway continuing to MT/V5 and the ventral pathway projecting into V4. 


\subsection{Overview of the Secondary Visual Cortex}

\subsubsection{Secondary Visual Cortex: Stripe compartments and functional organization}

Like V1, Area V2 possesses a high degree of neuronal organization based upon both histological architecture and functional preference. Neurons are organized into domains according to at least one visual stimulus preference. When V2 tissue is sectioned tangentially and processed with CO, a regular, repeating pattern of stripes emerges (Figure 1-6). This pattern consists of alternating regions of stained (CO dark; thick and thin) and unstained (little CO activity; pale) stripes, with every other stripe often being wider (or thicker) than the other stained

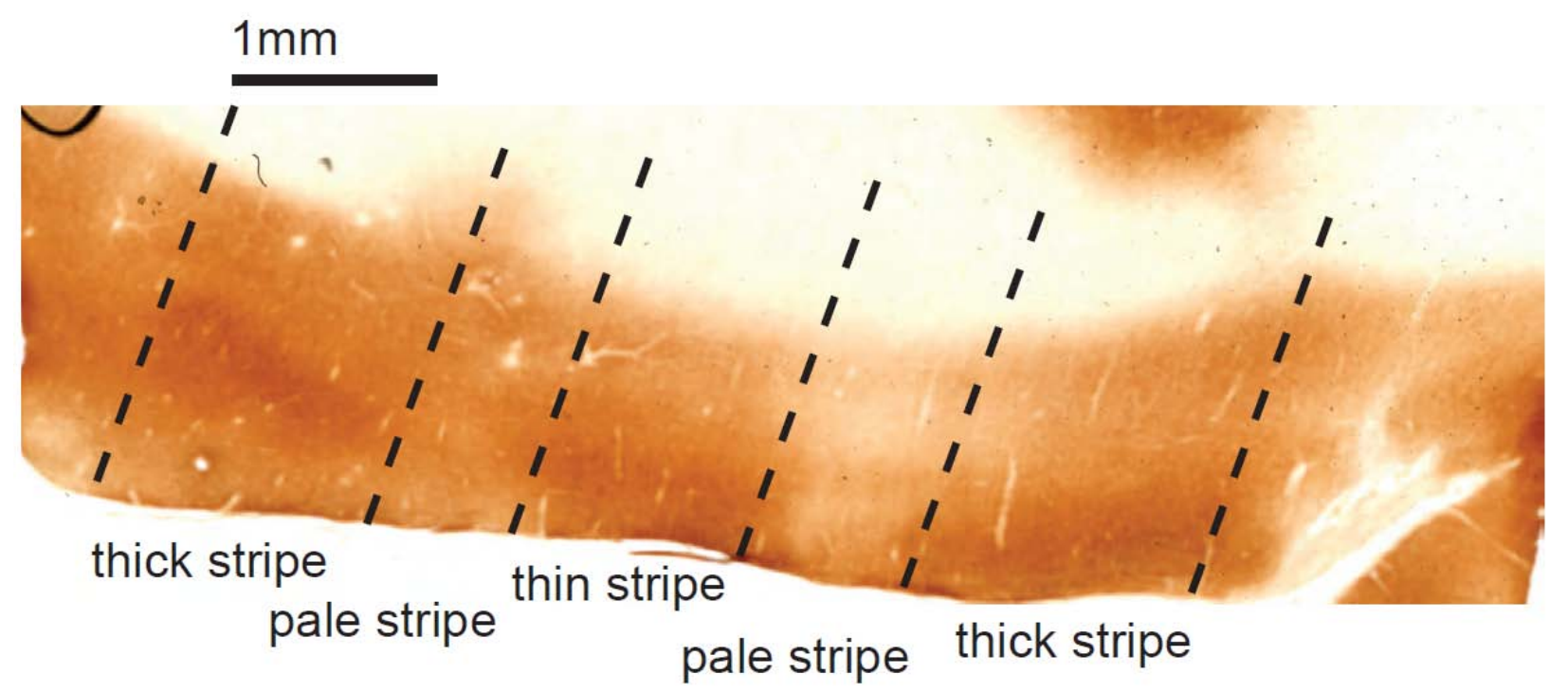

Figure 1-6. Stripe pattern of macaque secondary visual cortex revealed with cytochrome oxidase. The organization of the secondary visual cortex (V2) visualizes into a repeating stripe pattern when processed with cytochrome oxidase.

regions (thin), This pattern of thin stripe, pale stripe, thick stripe, pale stripe encompasses the entirety of V2 and runs perpendicular to the lunate sulcus and the V1/V2 area border. A single cycle of stripes (thin, pale, thick, pale) covers approximately $4 \mathrm{~mm}$ of cortex (Tootell and Hamilton, 1989), with 10-12 cycles spanning the entirety of V2 (Livingstone and Hubel, 1983; Horton, 1984; Hubel and Livingstone, 1987; Roe and Ts'o, 1995; Olavarria and Van Essen, 1997; Sincich and Horton, 2003). A similar striping pattern has been observed in processed human visual cortex (Sincich and Horton, 2005).

Within these stripes, V2 neurons seemingly organize into domains based upon certain distinct visual stimulus attributes or components. Domains within thin stripes typically exhibit preference for the surface properties of a visual stimulus - such as color or luminance; thick and 
pale stripes are organized according to the orientation of the stimulus. Initial experimental findings suggested that neurons located in the thin stripes have a preference for color and cells with an orientation preference reside in pale stripes (Hubel and Livingstone, 1987; Tootell and Hamilton, 1989; Levitt et al., 1994; Roe and Ts'o, 1995; Ts'o et al., 2001; Vanduffel et al., 2002; Xiao et al., 2003; Wang et al., 2006; Lu and Roe, 2008). Some reports suggest that color-oriented V2 neurons are more prevalent at the pale/thin stripe border and may show specific clustered organization (Roe and Ts'o, 1995). Color preferring areas within the thin stripes have been described as ranging in $1.4 \mathrm{~mm}$ long, $0.07-0.21-\mathrm{mm}$ wide to $0.52 \mathrm{~mm}$ long and $0.28 \mathrm{~mm}$ wide (Xiao et al., 2003). Electrophysiological, histological, double-labeled deoxyglucose, and optical imaging studies have supported the pattern that emerges with CO processing: discrete anatomical pale-thick-pale-thin pattern of V2 and a color selective cell organization in thin stripes and orientation selective cell organization in the pale and thick stripes (Ts'o et al., 2001; Vanduffel et al., 2002; Lu and Roe, 2008).

It is important to note that these descriptions of a stripe's properties are applied to the population of cells; a cell within the stripe can also possess other stimulus preferences. More recent electrophysiological findings suggest that a cell's color and/or orientation selectivity is not solely dependent on the stripe in which the cell is located (Gegenfurtner et al., 1996; Tamura et al., 1996; Kiper et al., 1997; Shipp and Zeki, 2002; Friedman et al., 2003; Gegenfurtner and Kiper, 2003). Neurons within orientation-preferring domains outside of the thin stripe can have a color preference in addition to an orientation preference. Conversely, cells within colorpreferring domains within the thin stripe may also exhibit orientation selectivity. Other studies have also indicated that cells with endspectral color preferences (e.g., blue, red) are less orientation selective than those preferring midspectral colors (e.g., yellow, green) or achromatic cells (Yoshioka and Dow, 1996), suggesting that "stripes” are organizations of cells arranged according to a particular preference (i.e. color-preferring cells in a thin stripe). Thus cluster stripe location and cluster preference do not appear to completely dictate preference at the level single neuron and cells with multiple preferences across all stripes are prevalent in V2 despite the presence of distinct functional domains.

In summary, V2 neurons are functionally organized into domains where groups of neurons share common preference characteristics in one visual stimulus dimension. However individual neurons within these groups may have diverse (and unshared) preferences in other 
visual stimulus dimensions. The functional organization of cells within the stripes, specifically the proximity of domains with differing preferences (i.e. color vs. orientation), in combination with the rich intra-areal connectivity between and within the stripes, makes V2 an excellent experimental model system to investigate the possible computational role of neighboring cluster organization (Section 1.3) These organizational properties of V2 can be applied and then investigated in computer modeling (Chapter 3). Mixed color/orientation response properties can be further characterized in V2 by single unit sampling in response to strategic color and/or oriented visual stimulation via in vivo electrophysiology studies (Chapter 4).

\subsubsection{Secondary Visual Cortex: Stripe Connectivity}

The functional role of the thick/pale/thin/pale stripe organization, juxtaposing domains of color- and orientation-preferring neurons, is not completely understood. The "Minimum Wiring Hypothesis" purports that neurons with similar response preferences and properties arise together through development: the close proximity of the cells occurs purposefully in order to reduce the length of the connections between them. While this hypothesis does not suggest a functional advantage to this arrangement, the evolutionary advantage and rationale are apparent through the maximization of cortical space (Koulakov and Chklovskii, 2001; Chen et al., 2006). A differing explanation for the domain organization of V2 examines the length of connections from a different perspective, based upon Hebbian mechanisms. Neurons that are in close proximity are more likely to have synaptic interactions and through repeated firing common connections and neurons with like-receptor fields would strengthen, giving rise to domains of cells with similar response characteristics (Abbott and Nelson, 2000; Song S et al., 2000). This type of domain organization may have functional advantages as topographic groupings amplify sensory signals by facilitating local reentrant mechanisms (Douglas et al., 1995) or promote further sharpening of response tuning curves (Gardner et al., 1999).

Throughout V1 and V2, there is a high degree of interconnectivity, both across cortical areas (inter-areal connections) and within a cortical area (intra-areal connections). Inter-areal connections have been commonly linked with the building of new combined properties while intra-areal connections have been commonly relegated to a modulatory or contextual role. In V1, horizontal axonal connections can traverse millimeters and often preferentially interact with regions of cells with similar preferences (Yoshioka et al., 1996). However, as far as $400 \mu \mathrm{m}$ from 
a neuron dense axon arbors have potential to intermingle and interact with many neurons with diverse functional properties; modified response properties would likely be a result of such proximity (Malach et al., 1993). Previous work in V1 has also demonstrated that a neuron's response can be modified based upon the context of its surround; this interaction was thought to result from surrounding cells and horizontal connections (Gilbert and Wiesel, 1990). There has been very little published work examining the role of intra-areal connections in V2 however. The close proximity of pale, thick, and thin stripes allows for more than adequate interactions between color and orientation preferring regions. The short range between regions would greatly increase the probability of such a meaningful interaction, despite the difference in overall preference of cells. Previous work has demonstrated that many cells within V2 possess dual selectivity to color and orientation (Tamura et al., 1996). This same work also documented correlated firings from cells of different and distinct preference populations, suggesting the potential for potent intra-areal integration and mixing of inputs within V2. Taken in concert, these findings suggest that connections between the domains of the pale and thin stripes (and their associated elevations in density of neurons with particular stimulus preferences) could be a potent mechanism to further enable the integration of color and orientation information. This mechanism may be even more probable due to the relatively proximity of these different domains across the thin/pale stripe border.

\subsubsection{Secondary Visual Cortex: Stripe Input from V1 and the Integration of Color and}

\section{Orientation Responses}

Despite probable mixing of parvo- and magnocellular streams prior to V2 (and probable supplementary inputs from konio- pathways as well), the pathways from V1 to V2 are thought to be relatively segregated and independent (Figure 1-7). Current studies of V1 and V2 connectivity appear to support a relatively minor role for V1 in significant color and form integration. Simultaneous recording of spike trains in V1 and V2 indicate that neurons in both areas respond to highly specific color and orientation tuning within relatively distinct compartments, although cross-correlation activity peaks indicate the presence of some interconnectivity or 'cross-talk' between compartment (Roe and Ts'o, 1999). The long-standing theories state the CO blobs, highly populated with color preferring cells, project to the thin stripes while interblob regions, dominated by orientation selective cells, send afferents to both pale and thick stripes of V2 
A.

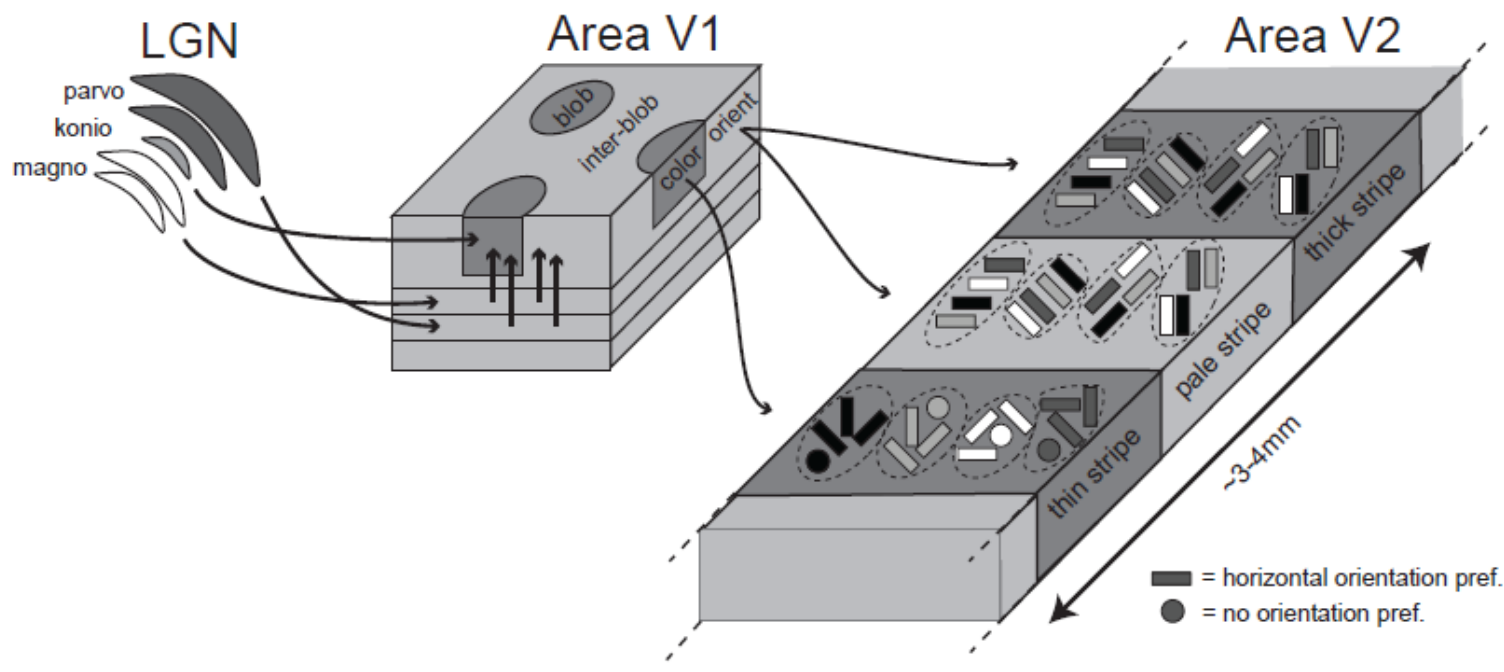

B.

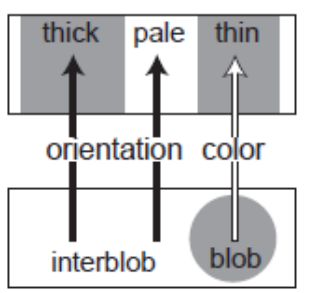

C.

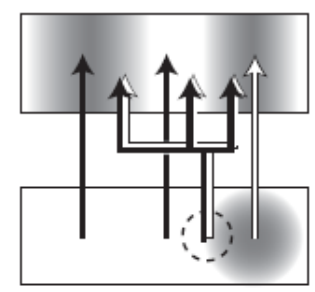

D.

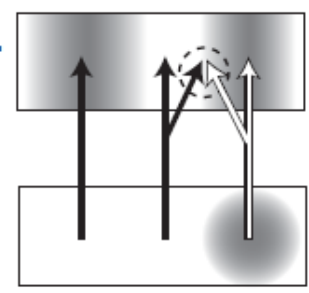

E

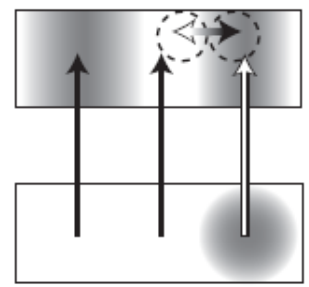

Figure 1-7. Putative circuits underlying integrative computation in primate Area V2 cortex. (A) Cytochrome oxidase staining delineates 'blob' and 'interblob' compartments in Area V1, and cycles of thin, thick and pale 'stripes' in V2. Lateral geniculate (LGN) projections and V1 circuitry have been thought to mediate color selectivity rather than orientation selectivity in blob neurons, and orientation selectivity rather than color selectivity in interblob neurons. In V2, neighboring thin stripe neurons share similar color preference (color domains, dotted idealized boundaries), whereas neighboring pale or thick stripe neurons share similar orientation preference (orientation domains). Bar orientation indicates orientation preference of idealized schematic neurons, shading indicates differential color preference. Many V2 neurons also exhibit combined color/orientation selectivities superimposed on this domain organization, and thus color and orientation preference is distributed across all stripes to some degree.

Some thin stripe neurons show poor orientation preference (shaded circles). How pervasive combined color/orientation preference might arise in V2 is unclear. Some experimental evidence indicates that blob neurons project onto thin stripes without contribution from interblob regions, $(B)$, reducing the potential for an inter-areal convergence of color and oriented streams. Other data indicate some color oriented cells do occur at the blob/interblob border, and possibly elsewhere in V1, and these might divergently project to all V2 stripes, (C). Some interblob and blob projections may converge at or near the thin/pale stripe border, $(D)$. Our hypothesis, $(E)$, builds on evidence that V2 intra-areal projections are surprisingly prevalent, spatially extensive and can support inter-stripe interaction. We suggest that these circuits could support efficient intra-areal integration of different (e.g. color and orientation) domain preferences. This domain -mediated computational mechanism could co-exist with all putative V1/V2 circuitries $(B-D)$, and could occur between other neighboring domain classes in V2 and beyond.

(Livingstone and Hubel, 1984b; Hubel and Livingstone, 1987; Sincich and Horton, 2002). Recent data supports the notion that blob to thin stripe projections are distinct from inter-blob to pale/thick stripes; the blob/thin stripe pathway is well known to be associated with color selectivity rather than orientation selectivity (Sincich and Horton, 2002). A hierarchical convergence mechanism underlying the color/oriented response would seem less likely if color 
and orientation information is transmitted separately via these distinct channels. These circuits instead would suggest that a neuron's combined selectivity for color and orientation could feasibly arise from the interaction of neurons within different stripe compartments of V2, via local horizontal (i.e. intra-areal) connections. Neuroanatomical tracer studies indicate that thin stripes may however receive inputs from both blob and interblob V1 regions, and these circuits could contribute to the color-orientation responses in and near the thin stripe (Xiao and Felleman, 2004). These data offer some support for inter-areal mechanisms combining separately represented information in V1 onto single color-oriented neurons in or at the border of thin stripes of V2.

Guided by imaging and histological correlates, previous studies used cross correlation single unit electrophysiology to identify diverse patterns of functional connectivity between V1 and V2 neurons (Roe and Ts'o, 1999). They found that non-oriented color cells in V1 exhibit functional connectivity with color-oriented neurons in V2 when their receptive fields overlap. This suggested a mechanism whereby projections from non-oriented color cells in V1 might contribute to the construction of color-oriented responses in and near the thin stripe in V2.

There are other possibilities however. A minority of V1 cells located at the blob/interblob border also exhibit mixed color/orientation preference (Landisman and Ts'o, 2002a, b) and these could divergently project to all stripes in V2. There is not a consensus yet for how feedforward projections from V1 would lead to color-oriented responses in V2. In part this may be because different groups have used varying stimulus paradigms, recording methods, and analysis approaches.

In summary, there is existing evidence that V2 intra-areal projections are extensive, and could support substantial interstripe interactions (Levitt et al., 1994; Malach et al., 1994; Tamura et al., 1996). Classical hierarchical models suggest that more complex neuronal selectivity and receptive fields are built via the converging projections from lower to higher areas. Local circuitry, on the other hand, has usually been associated with contextual or inhibitory response modulations rather than convergent mechanisms per se (Gilbert, 1983; Callaway, 1998). The intrinsic connections and interactions between the highly organized domains of the thin and pale stripes in V2 could possibly support or further enhance the mixing of color/oriented preferences. 


\subsection{The Role of Clustered Organization in Cortical Sensory Processing}

This dissertation focuses on the possible role of functional domains in V2 comprising neurons of similar visual stimulus preference and this section reviews some of the pertinent general theories regarding clustered neuronal organization. The neocortex is a highly evolved and complex, dynamical system comprised of recurrent interconnections and interactions that likely occur at a number of spatial and temporal scales (Edelman, 1987; Felleman and Van Essen, 1991). Considering this, the probable convergence and divergence of projections among cortical areas are therefore likely to collectively produce a dominantly constructive interaction. Neurons located in close proximity are more likely to fire together (Zohary et al., 1994; Douglas et al., 1995; Constantinidis and Goldman-Rakic, 2002). The pooling of neural response by a population theoretically enhances the signal-to-noise ratio as the number of (similarly responsive) neurons in a population increases, but the benefit of population on signal-to-noise ratio depends on the correlation co-efficient of firing amongst the cells of the population. This is an argument for why clustering can be helpful in processing and why only a finite number of connections are useful, as Zohary et al.'s data suggest an asymptotic correlation between the number of neurons and signal-to-noise ratio (Zohary et al., 1994). This may be especially so in higher order sensory areas such as Areas V2 and V4 where functional domains are relatively large, where adjacent functional domains can map substantially different sensory phenomenon, and where intrinsic outputs are more extensive and are known to be less specific (cf. like-to-like connectivity well described in Area V1) (Amir et al., 1993; Malach et al., 1994; Tamura et al., 1996; Ghose and Ts'o, 1997).

This theory of clustering is not unlike the phenomenon of "scale-free graphs" (Barabasi and Albert, 1999). This type of network consists of topologies that exhibit power law distributions in the connectivity of network nodes. In essence, these nodes are "hubs" that interact with other variables (e.g. cells). Larger hubs exude a greater influence but are less common; smaller hubs are more prevalent but less influential. If a hub was to cease, it would likely be a smaller one therefore maintaining the connectedness of the network since the larger hubs would still remain (Amaral et al., 2000). The nodes are also highly dictated by a clustering coefficient, or the tendency of nodes to be clustered together. In scale free networks, there is a high probability two nodes randomly associating (Watts et al., 1998). With regards to V2 preference distribution, this theory would suggest that a node associated with one particular 
group of cells (e.g. color-preference cells) would likely interact with a separate, differing node and group of cells (e.g. orientation-preference cells). 


\subsection{Temporal Response Characteristics of V1 and V2: Latency}

Relatively little experimental data has been gathered concerning the temporal response characteristics of neurons in the second visual cortical area of the primate V2. One important response parameter, latency, is defined here as the time from visual stimulus presentation to a significant modification in a single visually responsive neuron's spiking activity.

\subsubsection{Documented Latencies}

Some of the earliest studies used light and dark bars of optimal length and orientation to report mean latency times of $85 \mathrm{~ms}$ in V1 and $96 \mathrm{~ms}$ in V2, however only a relatively few number of cells were observed (Raiguel et al., 1989). These times were subsequently verified and further described based upon the cortical layers: the shortest latencies were found in the middle cortical layers, these latencies increased moving outward to the supra- and infragranular layers in both V1 and V2 (Nowak et al., 1995). Other studies have confirmed these latencies (Figure 1-8) (Maunsell and Gibson, 1992; Nowak and Bullier, 1998; Schmolesky et al., 1998; Marcus and Van Essen, 2002) however these latency studies often failed to fully characterize the stimulus preferences of a neuron. Orientation was typically tested at the preferred angle, via

\section{RESPONSE LATENCY}

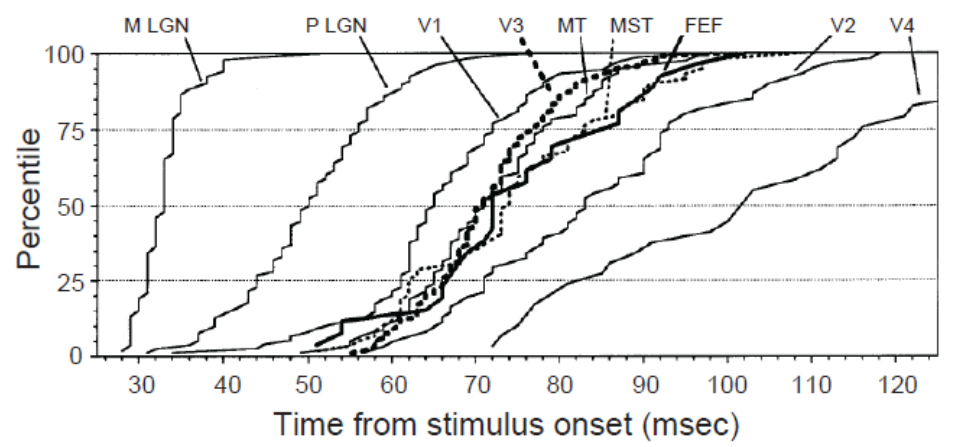

Schmolesky et al., 1998
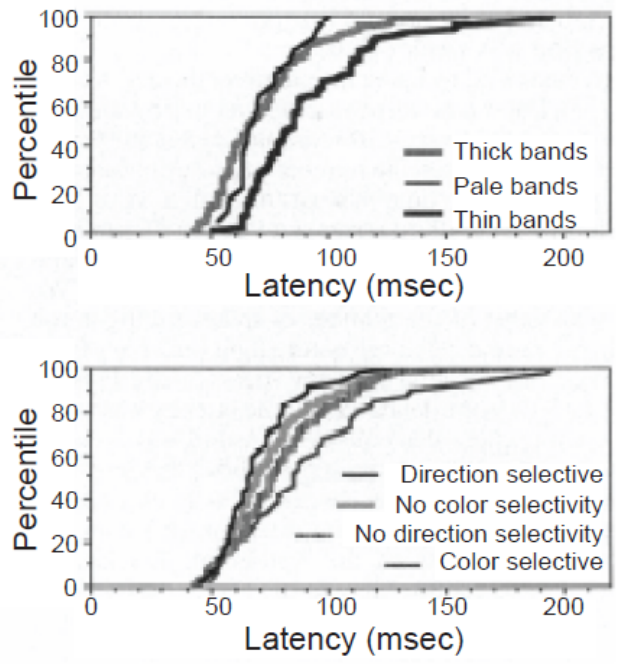

Munk et al., 1995

Figure 1-8. Observed latencies of the primate visual system. Previous studies have investigated the response latencies in the non-human primate visual areas. The range of latencies vary considerably, with earlier areas displaying a shorter latency comparatively to the long latencies of higher cortical areas. Stripe location and neuron preference also affect latency, with thin bands and color selective cells possessing longer latencies. This figure is adapted from other sources (Schmolesky et al., 1998; Munk et al., 1995). 
achromatic bar presentation, and few, if any, other stimulus attributes (e.g., luminance contrast, color, size) were systematically compared. This historical emphasis on preferred achromatic orientation may have masked subtle yet significant differences in temporal response that occur when other stimulus attributes are also presented. Studies of V1 cells have shown that some stimulus components, such as the spatial frequency and orientation, are largely separate preferences within a single neuron. However, certain combinations of spatial frequency and orientation are inseparable and when used concurrently, yield significantly modified latencies (Mazer et al., 2002).

Previous studies have examined the latencies of neurons in the pale, thick, and thin bands of the macaque V2 and these findings suggest that neurons within thin bands tend to have longer latencies than neurons in the other band types (thick and pale stripes). Furthermore a systematic examination of a cell's stimulus preference on latency revealed that color-selective neurons have longer latencies than non-color-selective cells and non-orientation-selective neurons have longer latencies than orientation selective (Munk et al., 1995). However, this study was in most part a multi-unit (rather than single unit) experimental design. Multi-unit design collects response activity simultaneously from multiple rather than single neurons at one electrode site and while yielding a higher aggregate spike registration, their interpretation is somewhat limited because individual V2 neuronal responses cannot be differentiated, characterized and analyzed. In addition, this study primarily used qualitative rather than quantitative means to determine tuning preference. These latency calculations were primarily comparisons between cells using a common stimulus and did not comprehensively examine a single cell's latency using varying visual stimuli let alone the optimal one. Detailed, comprehensive temporal characterizations of color vs. orientation response in V2 remain to be investigated, which is motivation for the study presented in Chapter 4.

Previous investigations in V2 have also often overlooked the preferred color and/or orientation preference when characterizing latency and other temporal responses. The importance of a using a cell's preferred orientation has long been known: the latency of a neuron increases when presented with a non-optimal orientation bar (Celebrini et al., 1993). However previous studies have often claimed there is no significant difference in a cell's latency for different color presentations. A relatively limited range of colors were presented in these studies 
since color-dependent latency was not the focus of the studies (Creutzfeldt et al., 1987; Munk et al., 1995).

In summary, the quantitative characterization of single V2 neuronal temporal response to colored vs. achromatic oriented stimuli is not well studied. By utilizing a broader, more controlled range of colored and oriented stimuli and more precisely determining a cell's color preference, it may be possible to determine color-dependent differences in latency (and other temporal response characteristics). Changes in the background luminance have a significant modulatory effect on latency to foreground stimulus presentations (Huang and Paradiso, 2005), so it may be feasible to reveal modulations in latency when a stimulus bar is presented at the preferred orientation. This provides the rationale for the stimuli used in Chapter 4.

\subsubsection{Circuitry and Mechanisms Associated With Observed Latencies}

By better characterizing the latencies and differential temporal responses of separate and combined color and orientation stimulation of a neuron, one may be able to infer neuronal circuitry (Anzai et al., 2007). Groups such as Raiguel et al. (1989) and Maunsell and Gibson (1992) have gathered data suggesting neuronal latencies of "higher" cortical area are on average longer than "lower" cortical areas, consistent with the notion of a general progression of activation through a serial areal hierarchy. A cell delivering two different latencies for two different facets of visual stimulation is consistent with two separate parallel pathways of innervation converging onto that cell. For example, segregated pathways for color and orientation in V1 as proposed by Hubel and Livingstone might be eventually convergent in some higher cortical areas, and thus latencies recorded from the tertiary convergent cell might be expected to shift as color is introduced to an achromatic stimulus. (Livingstone and Hubel, 1984b; Hubel and Livingstone, 1987). Alternately, these differences in latency times may also be attributed to interactions with other cortical areas or local feedback within V2 (Douglas et al., 1995). These potential separate pathways suggested by the latency times would also be at the level of a single neuron (e.g. a neuron within a pale stripe, or a color-selective neuron). Understanding latencies of neurons in response to stimuli are also thought to be important factors in attention models (Oram et al., 2002) and rapid behavioral responses (VanRullen et al., 2005). Further comprehensive quantitative characterization of V2 response latency in response to the systematic addition of stimulus features (such as supplementing an oriented stimulus with color) 
may offer useful information regarding mechanisms underlying the neural integration of visual stimulus properties. 


\subsection{Visual Stimuli}

\subsubsection{DLK Color Space}

There are several co-ordinate systems for the systematic specification of color stimuli in vision research and the 'DLK' co-ordinate system, or 'color space,' is one of the more common. The DLK color coordinate system is named for the investigators who developed it: Derrington, Lennie, and Krauskopf (Derrington et al., 1984). This system is a three dimensional color space, originally developed to further characterize LGN neurons. Red/green (associated with the L- and M-cone inputs) maps along one axis, blue/yellow ( $\mathrm{S}-$ and ( $+\mathrm{M}-$ ) cones) along the second axis, and luminance along the third axis. Various coordinate locations within this color space correspond to different hues: red/green values are manipulated along the $\mathrm{X}$-axis, blue/yellow on the $\mathrm{Y}$-axis, and luminance on the Z-axis. If stimulus modulation is kept along a single plane (i.e. the $\mathrm{X} / \mathrm{Y}$ axes only or equal luminance about a white point), this implies a constant linear summation of inputs with only changes in the relative proportions amount of red, green, yellow, and blue cone activation. A more complicated, nonlinear summation occurs when cells' preferences lie outside of the isoluminant plane (i.e. X, Y and Z coordinate values or variations in red, green, blue, yellow, and luminance). Cells characterized within the LGN exhibited linear responses along the red/green and blue/yellow axes only (Derrington et al., 1984). In V1, some cells also displayed maximal firing along these two axes but most did not (Lennie et al., 1990). This suggests the majority of V1 neurons do not resemble the clearly defined red/green or blue/yellow cells seen in the LGN, and suggest further mixing of cone inputs. In many instances a V1 neuron's response increased nonlinearly with changes in luminance, as one would expect if that cell's preference was located away from the isoluminant plane (i.e. possessing values in all three axes). Though defined for use initially in the investigation of visual processing in the LGN, the DLK color coordinate is one of the more common systems used when developing colored stimuli for studying vision in primate V1 and V2. Other notable color systems in vision research include the Munsell system (based on hue, value, and chroma) and CIE 1931 (based on measurements of human perception).

\subsubsection{Linear vs. Non Linear Processing in Visual Neurons}

In addition to an elementary stimulus consisting of a single visual component (e.g. a color field or an achromatic orientation bar), recent research suggests that many extrastriate cells are 
responsive to combinatorial visual stimuli. These combinatorial stimuli, for example, may consist of two separate, differently oriented bars conjoined to create an angled or "elbow” joint stimulus (Ito and Komatsu, 2004) or the combination of more than one single visual attribute, such as color and orientation. Many of the V2 neurons' responses to the elbow stimuli were greater than the linear summation of the component orientation bars displayed individually. The superposition property as applied to neural electrophysiology indicates that if the net activity response of a cell as a result of two or more simultaneous stimuli is the sum of the responses which would have been evoked by each stimulus individually and separately, linear summation has occurred. In contrast, if the net response of a cell from multiple stimuli is not equal to the sum of the individual stimuli, that is characteristic of a non-linear summation. The stimulus components can be separated into distinct and disjoined orientation bars within the receptive field (in some instances by a relatively large distance) yet still evoke the optimal (and non-linear) response from the cell (Anzai et al., 2007).

S-cone stimulation evokes significantly longer latencies in V1 than those from L- or Mcone stimulation. Yet upon reaching V1 all LMS cone component information is integrated, suggesting that recurrent networks may need to non-linearly amplify a weaker and delayed Scone signal (Cottaris and De Valois, 1998). In V1 most cells found in the superficial layers of the cortex displayed linear summation of cone input; however some V1 cells exhibited sharply tuned responses suggestive of a non-linear cone summation process (Lennie et al., 1990). A larger proportion of V2 cells (about one third) appear highly selective for one color which is not consistent with a linear cone summation pattern, however (Kiper et al, 1997). Non-linear processing of visual stimulus components and attributes thus appear to be quite prevalent by V2, and any integration of color and orientation attributes in V2 may potentially involve a non-linear summation of individual component/attribute contribution, and this may vary across the temporal response in time as reported in higher areas such as V4 (Brincat and Connor, 2006). 


\subsection{Sensory Processing Modeling}

\subsubsection{Previous Visual Cortex Models}

The modeling of neuronal activity and sensory processing is a well established tool in neuroscience, and has been commonly used to investigate signal processing in cortex-like systems, especially in V1 (Linsker, 1986c, b, a; Miller et al., 1989). The size, scale, and scope of cortical modeling vary widely, and are primarily selected on the nature of the scientific hypothesis being investigated. The implementation of large scale network require significant computational power, and thus early model designs were often constrained to individual or small networks of neurons (Hines and Carnevale, 1997; Bower and Beeman, 1998). More recently, investigators have implemented larger scale models capable of representing a two dimensional sheet of cortical tissue, accounting for extra-cortical outputs, inputs and defined patterns of intracortical connectivities (Bednar et al., 2004). Models of large cortical networks comprised of many interconnected neurons are an effective way to investigate their individual or combined physiological functions. The resulting interconnectivity yields cortical maps containing systematic two-dimensional, topography of features and preferences, such as retinotopy or line orientation and motion direction (Blasdel, 1992). Recent advances have shown that the basis of V1 models can be applied to understanding the organization of the thin, thick, and pale stripes in V2 cortex (Swindale, 2007). Modeling is an advantageous supplement to in vivo experimental data collection because model data can lend credence to hypotheses formed from preliminary research and suggest future avenues to explore. The organization and application of computational models and analytical tools to biologically-inspired computing designs addresses these issues. Specifically, there is a need for improved knowledge of the relative roles of interareal vs. intra-areal cortical circuitry. Improved understanding of the comparative functions of clustered vs. distributed organizations in a cortical computation model can further the knowledge of neuronal interaction.

By collecting the necessary biological data, it would be possible to further develop these existing models, to optimally "recreate" aspects of the physiology and anatomy of V2, and better understand how the collective actions of neuronal groups and their inter-areal connections are able to affect the response of a single neuron. In turn, these simulations can usefully guide other in vivo experiments. More advanced models are necessary, however, and must be further 
developed to better emulate the complexities of the primate cortex, as addressed in the next section.

\subsubsection{Common Design Features in Cortical Network Models}

The processings of early neurosensory systems can be likened to a form of efficient coding. This hypothesis developed by Horace Barlow in 1961 purports that neurons encode and transmit information within a minimal number of spikes. Experimental and theoretical data have supported this theory of elegant design (Olshausen and Field, 1997), and therefore a computational analogue of neuronal spike timing is commonly used as a dependent model variable.

Many modeling studies have been primarily motivated by an interest the inherent development of cortical organization (e.g. functional topography), and thus these models have been configured with features appropriate to that inquiry. Certain programs and models currently exist that explore the self-organizing cortex-like networks with specific intracortical connections and their probabilities (Bednar et al., 2004; Cooper and Ramsden, 2006). It has been suggested that organized functional mappings may be a residue of developmental cortical competition (Kohonen, 1982; Piepenbrock and Obermayer, 2000), the consequence of a need for an optimal and uniform 'coverage' of functional representations (Swindale et al., 2000), or a prevailing need for the cortex to simply minimize wiring length (Koulakov and Chklovskii, 2001; Chen et al., 2006).

One of the key principles suggested underpinning cortical organization and interconnectivity - and therefore a vital attribute in many developmental cortical models - is the wirelength minimization hypothesis. This hypothesis proposes that cortical topography and interconnectivity patterns are optimally arranged to minimize the total volume/length of necessary neuronal projections (e.g. axons and dendrites) for optimal spatial configuration. In other words, suggesting that connections are likely to occur between regions of the cortex with similar response properties and creating continuity across differing preference regions (Mitchison, 1991, 1995). The increased densities at one location, as would be dictated by the wirelength minimization hypothesis, could give rise to higher probability of synapse formation (Stepanyants and Chklovskii, 2005). Thus many model connectivity implementations have been 
configured with wirelength minimization as a fundamental (theoretically inspired) design constraint.

While model connectivity designs can be based on such theoretically derived constraints as wirelength minimization, there nevertheless is a substantial body of experimentally derived data that describes how cortex is organized and interconnected. Each neuron is known to have numerous dendrites and (typically) a single axon emanating from it, creating a complex and intricate set of interactions with other neurons in proximal and distal cortical regions. Two common ways to model intra-areal cortical connectivity and organization are radially diffuse distribution of projections in the immediate vicinity of the original neuron and patchy distributions that can extend up to several millimeters away (Amir et al., 1993; Lund et al., 1993). The radial diffuse approach is a conservative estimate of the distribution in which the spatial density of the projected terminals decrease radially and equally in all directions (Sholl, 1953; Abeles, 1991). Although this form of homogenous radial falloff is not precisely consistent with reported terminal configurations, it has been commonly used to sufficiently represent neuronal projections in relatively recent cortex network models (Stemmler et al., 1995; Spiridon and Gerstner, 2001; Kang et al., 2003). A non homogenous radial falloff in local intra-cortical connectivity is well described in primate cortex, however. For example, a patchy rather than an exclusively homogenous radial distribution of terminals is obtained when small injections of biocytin tracer are placed and subsequently tracked in macaque cortex V2 (Van Essen et al., 1986; Coogan and Van Essen, 1996). In macaque V2, small local biocytin injections (breadth about $500 \mu \mathrm{m}$ or less) tend to project a proportion of their terminals to the a finite set of 'patches' $0.5-2 \mathrm{~mm}$ from the injection site (Amir et al., 1993) (Figure 1-9). They found that patchy distributions account for a percentage of terminals projecting a distance (400 - $2100 \mu \mathrm{m})$ from a particular V2 location. Terminal distributions appeared to be relatively uniformly distributed within individual patches (typically about $200 \mu \mathrm{m}$ in diameter). Patchy projections are not necessarily consistent with simple diffuse homogenous models associated with wirelength minimization implementations. Thus few models have explicitly incorporated such patchy projections in their designs to date. 


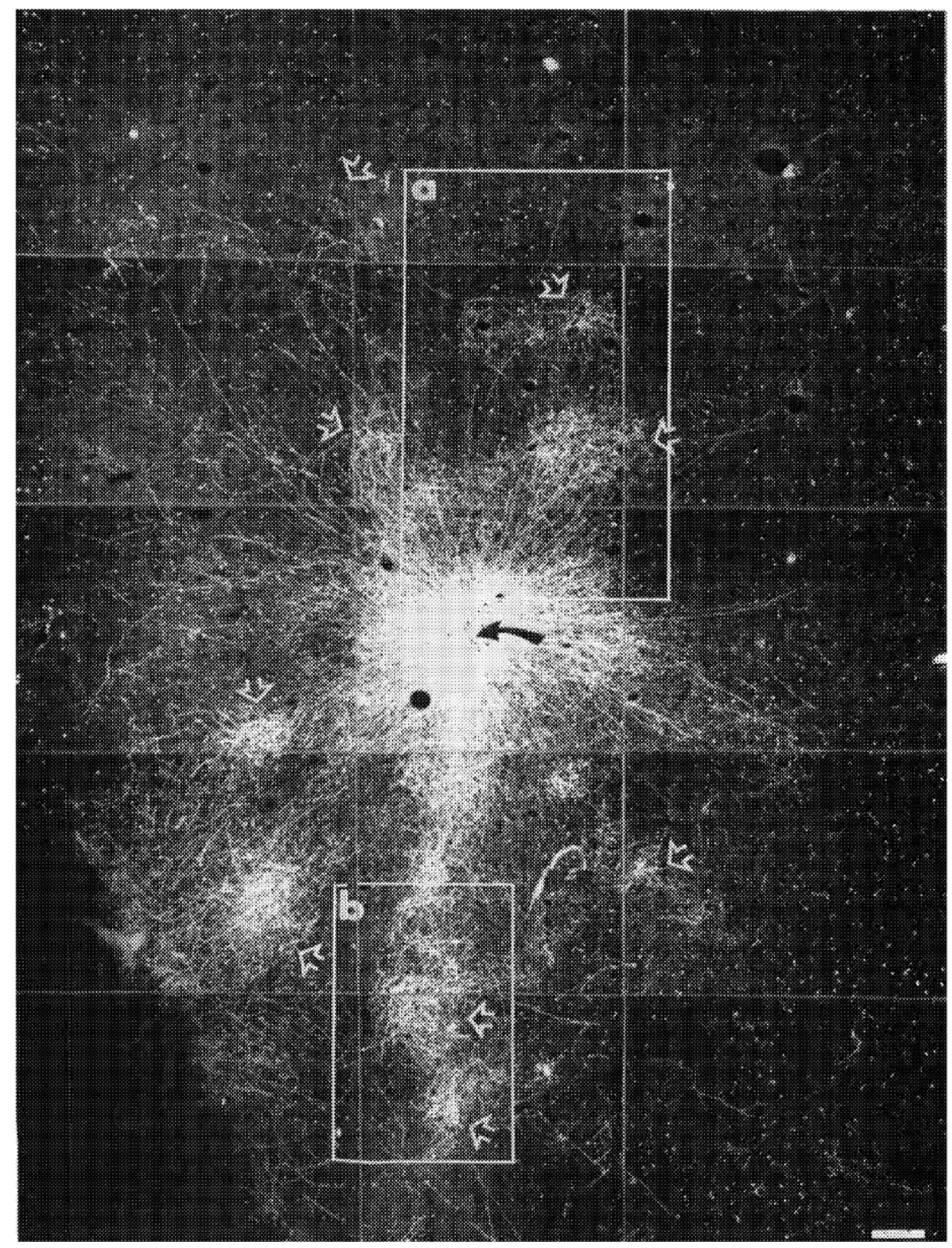

Figure 1-9. Examples of clusters in the secondary visual cortex. Intrinsic fibers were labeled by a 230 micron diameter injection biocytin at the arrow, illustrating a network of fibers and the patches located away from the injection site. This figure is adapted from other sources (Amir et al., 1993). 


\subsection{Conclusions}

This chapter outlined the flow visual information from the eye to V2, specifically focusing on the organization of neurons in this visual area according to response preference. This particular area of visual cortex has documented organizations of neurons clustered according to color and orientation, providing an excellent model to investigate functional domains and their role in cells with mixed and multiple preferences. This chapter also discussed the role of computer modeling and single unit electrophysiology to explore this topic of integration of color and orientation responses on V2. The information presented here provides a basis for the objectives and rationale of this dissertation (Chapter 2) and a background for the modeling (Chapter 3) and electrophysiology (Chapter 4) experiments discussed here. 


\subsection{References}

Abeles M (1991) Corticonics: Neural Circuits of the Cerebral Cortex. Cambridge: Cambridge University Press.

Allman JM (1999) Evolving brains. New York: Scientific American Library: Distributed by W.H. Freeman and Co.

Amaral LA, Scala A, Barthelemy M, Stanley HE (2000) Classes of small-world networks. Proc Natl Acad Sci U S A 97:11149-11152.

Amir Y, Harel M, Malach R (1993) Cortical hierarchy reflected in the organization of intrinsic connections in macaque monkey visual cortex. J Comp Neurol 334:19-46.

Anzai A, Peng X, Van Essen DC (2007) Neurons in monkey visual area V2 encode combinations of orientations. Nat Neurosci 10:1313-1321.

Barabasi AL, Albert R (1999) Emergence of scaling in random networks. Science 286:509-512.

Barishak YR (1992) Embryology of the eye and its adnexae. Dev Ophthalmol 24:1-142.

Bednar JA, Kelkar A, Miikkulainen R (2004) Scaling self-organizing maps to model large cortical networks. Neuroinformatics 2:275-302.

Blasdel GG (1992) Orientation selectivity, preference, and continuity in monkey striate cortex. J Neurosci 12:3139-3161.

Blasdel GG (1992) Differential imaging of ocular dominance and orientation selectivity in monkey striate cortex. J Neurosci 12:3115-3138.

Borwein B (1983) Scanning electron microscopy of monkey foveal photoreceptors. Anat Rec 205:363-373.

Bower JM, Beeman D (1998) The Book of GENESIS: Exploring Realistic Neural Models with the GEneral NEural SImulation System. Santa Clara, CA: Telos.

Brincat SL, Connor CE (2006) Dynamic shape synthesis in posterior inferotemporal cortex. Neuron 49:17-24.

Brodmann K (1905) Beitrage zur histologischen Localisation der Grosshirnrinde. J Psychol Neurol 46:369-384.

Brown PK, Wald G (1964) Visual Pigments in Single Rods and Cones of the Human Retina. Direct Measurements Reveal Mechanisms of Human Night and Color Vision. Science 144:45-52. 
Callaway EM (1998) Local circuits in primary visual cortex of the macaque monkey. Annu Rev Neurosci 21:47-74.

Casagrande VA, Xu X, Sary G (2002) Static and dynamic views of visual cortical organization. Prog Brain Res 136:389-408.

Celebrini S, Thorpe S, Trotter Y, Imbert M (1993) Dynamics of orientation coding in area V1 of the awake primate. Vis Neurosci 10:811-825.

Chalupa LM, Lia B (1991) The nasotemporal division of retinal ganglion cells with crossed and uncrossed projections in the fetal rhesus monkey. J Neurosci 11:191-202.

Chatterjee S, Callaway EM (2003) Parallel colour-opponent pathways to primary visual cortex. Nature 426:668-671.

Chen BL, Hall DH, Chklovskii DB (2006) Wiring optimization can relate neuronal structure and function. Proc Natl Acad Sci U S A 103:4723-4728.

Constantinidis C, Goldman-Rakic PS (2002) Correlated discharges among putative pyramidal neurons and interneurons in the primate prefrontal cortex. J Neurophysiol 88:3487-3497.

Coogan TA, Van Essen DC (1996) Development of connections within and between areas V1 and V2 of macaque monkeys. J Comp Neurol 372:327-342.

Cooper CG, Ramsden BM (2006) Color meets orientation: cluster organization probabilities in area V2 of macaque visual cortex. Society for Neuroscience: Atlanta, GA Neuroscience Meeting Planner:Program No. 546.510/U520, Online.

Cottaris NP, De Valois RL (1998) Temporal dynamics of chromatic tuning in macaque primary visual cortex. Nature 395:896-900.

Creutzfeldt OD, Weber H, Tanaka M, Lee BB (1987) Neuronal representation of spectral and spatial stimulus aspects in foveal and parafoveal area 17 of the awake monkey. Exp Brain Res 68:541-564.

Curcio CA, Sloan KR, Kalina RE, Hendrickson AE (1990) Human photoreceptor topography. J Comp Neurol 292:497-523.

Curcio CA, Sloan KR, Jr., Packer O, Hendrickson AE, Kalina RE (1987) Distribution of cones in human and monkey retina: individual variability and radial asymmetry. Science 236:579582.

Day RH, Kasperczyk RT (1983) Illusory contours in line patterns with apparent depth due to either perspective or overlay. Perception 12:485-490. 
de Monasterio FM (1978a) Properties of ganglion cells with atypical receptive-field organization in retina of macaques. J Neurophysiol 41:1435-1449.

de Monasterio FM (1978b) Center and surround mechanisms of opponent-color X and Y ganglion cells of retina of macaques. J Neurophysiol 41:1418-1434.

De Valois RL, Smith CJ, Kitai ST, Karoly AJ (1958) Response of single cells in monkey lateral geniculate nucleus to monochromatic light. Science 127:238-239.

Derrington AM, Krauskopf J, Lennie P (1984) Chromatic mechanisms in lateral geniculate nucleus of macaque. J Physiol 357:241-265.

Dodd JV, Krug K, Cumming BG, Parker AJ (2001) Perceptually bistable three-dimensional figures evoke high choice probabilities in cortical area MT. J Neurosci 21:4809-4821.

Douglas RJ, Koch C, Mahowald M, Martin KA, Suarez HH (1995) Recurrent excitation in neocortical circuits. Science 269:981-985.

Dow BM, Vautin RG (1987) Horizontal segregation of color information in the middle layers of foveal striate cortex. J Neurophysiol 57:712-739.

Dowling JE, Boycott BB (1966) Organization of the primate retina: electron microscopy. Proc R Soc Lond B Biol Sci 166:80-111.

Edelman GM (1987) Neural Darwinism: The Theory of Neuronal Group Selection. New York: Basic Books.

Elston GN, Rosa MG (1998) Morphological variation of layer III pyramidal neurones in the occipitotemporal pathway of the macaque monkey visual cortex. Cereb Cortex 8:278294.

Erwin E, Baker FH, Busen WF, Malpeli JG (1999) Relationship between laminar topology and retinotopy in the rhesus lateral geniculate nucleus: results from a functional atlas. J Comp Neurol 407:92-102.

Essock EA, DeFord JK, Hansen BC, Sinai MJ (2003) Oblique stimuli are seen best (not worst!) in naturalistic broad-band stimuli: a horizontal effect. Vision Res 43:1329-1335.

Felleman DJ, Van Essen DC (1991) Distributed hierarchical processing in the primate cerebral cortex. Cereb Cortex 1:1-47.

Friedman HS, Zhou H, von der Heydt R (2003) The coding of uniform colour figures in monkey visual cortex. J Physiol 548:593-613.

Gardner JL, Anzai A, Ohzawa I, Freeman RD (1999) Linear and nonlinear contributions to orientation tuning of simple cells in the cat's striate cortex. Vis Neurosci 16:1115-1121. 
Gazzaniga MS, Ivry R, Mangun GR (2002) Cognitive Neuroscience: The Biology of the Mind, 2nd Edition Edition: W.W. Norton.

Gegenfurtner KR, Kiper DC (2003) Color vision. Annu Rev Neurosci 26:181-206.

Gegenfurtner KR, Kiper DC, Fenstemaker SB (1996) Processing of color, form, and motion in macaque area V2. Vis Neurosci 13:161-172.

Ghose GM, Ts'o DY (1997) Form processing modules in primate area V4. J Neurophysiol 77:2191-2196.

Gilbert CD (1983) Microcircuitry of the visual cortex. Annu Rev Neurosci 6:217-247.

Gilbert CD, Wiesel TN (1990) The influence of contextual stimuli on the orientation selectivity of cells in primary visual cortex of the cat. Vision Res 30:1689-1701.

Goodale MA, Milner AD (1992) Separate visual pathways for perception and action. Trends Neurosci 15:20-25.

Hanazawa A, Mikami A, Sulistyo Angelika P, Takenaka O, Goto S, Onishi A, Koike S, Yamamori T, Kato K, Kondo A, Suryobroto B, Farajallah A, Komatsu H (2001) Electroretinogram analysis of relative spectral sensitivity in genetically identified dichromatic macaques. Proc Natl Acad Sci U S A 98:8124-8127.

Hansen BC, Essock EA (2004) A horizontal bias in human visual processing of orientation and its correspondence to the structural components of natural scenes. J Vis 4:1044-1060.

Hendry SH, Reid RC (2000) The koniocellular pathway in primate vision. Annu Rev Neurosci 23:127-153.

Hines ML, Carnevale NT (1997) The NEURON simulation environment. Neural Comput 9:1179-1209.

Hinkle DA, Connor CE (2005) Quantitative characterization of disparity tuning in ventral pathway area V4. J Neurophysiol 94:2726-2737.

Horton JC (1984) Cytochrome oxidase patches: a new cytoarchitectonic feature of monkey visual cortex. Philos Trans R Soc Lond B Biol Sci 304:199-253.

Huang X, Paradiso MA (2005) Background changes delay information represented in macaque V1 neurons. J Neurophysiol 94:4314-4330.

Hubel DH, Wiesel TN (1968) Receptive fields and functional architecture of monkey striate cortex. J Physiol 195:215-243. 
Hubel DH, Wiesel TN (1969) Anatomical demonstration of columns in the monkey striate cortex. Nature 221:747-750.

Hubel DH, Wiesel TN (1974) Uniformity of monkey striate cortex: a parallel relationship between field size, scatter, and magnification factor. J Comp Neurol 158:295-305.

Hubel DH, Wiesel TN (1977) Ferrier lecture. Functional architecture of macaque monkey visual cortex. Proc R Soc Lond B Biol Sci 198:1-59.

Hubel DH, Livingstone MS (1987) Segregation of form, color, and stereopsis in primate area 18. J Neurosci 7:3378-3415.

Hurwitz RL, Bunt-Milam AH, Chang ML, Beavo JA (1985) cGMP phosphodiesterase in rod and cone outer segments of the retina. J Biol Chem 260:568-573.

Ito M, Komatsu H (2004) Representation of angles embedded within contour stimuli in area V2 of macaque monkeys. J Neurosci 24:3313-3324.

Johnson EN, Hawken MJ, Shapley R (2001) The spatial transformation of color in the primary visual cortex of the macaque monkey. Nat Neurosci 4:409-416.

Kang K, Shelley M, Sompolinsky H (2003) Mexican hats and pinwheels in visual cortex. Proc Natl Acad Sci U S A 100:2848-2853.

Kanwisher N, McDermott J, Chun MM (1997) The fusiform face area: a module in human extrastriate cortex specialized for face perception. J Neurosci 17:4302-4311.

Kiper DC, Fenstemaker SB, Gegenfurtner KR (1997) Chromatic properties of neurons in macaque area V2. Vis Neurosci 14:1061-1072.

Kohonen T (1982) Self-organized formation of topologically correct feature maps. Biological Cybernetics 43:59-69.

Koulakov AA, Chklovskii DB (2001) Orientation preference patterns in mammalian visual cortex: a wire length minimization approach. Neuron 29:519-527.

Landisman CE, Ts'o DY (2002a) Color processing in macaque striate cortex: electrophysiological properties. J Neurophysiol 87:3138-3151.

Landisman CE, Ts'o DY (2002b) Color processing in macaque striate cortex: relationships to ocular dominance, cytochrome oxidase, and orientation. J Neurophysiol 87:3126-3137.

Lennie P, Krauskopf J, Sclar G (1990) Chromatic mechanisms in striate cortex of macaque. J Neurosci 10:649-669. 
Leventhal AG, Thompson KG, Liu D, Zhou Y, Ault SJ (1995) Concomitant sensitivity to orientation, direction, and color of cells in layers 2 , 3, and 4 of monkey striate cortex. $\mathrm{J}$ Neurosci 15:1808-1818.

Levitt JB, Kiper DC, Movshon JA (1994) Receptive fields and functional architecture of macaque V2. J Neurophysiol 71:2517-2542.

Linsker R (1986a) From basic network principles to neural architecture: emergence of spatialopponent cells. Proc Natl Acad Sci U S A 83:7508-7512.

Linsker R (1986b) From basic network principles to neural architecture: emergence of orientation-selective cells. Proc Natl Acad Sci U S A 83:8390-8394.

Linsker R (1986c) From basic network principles to neural architecture: emergence of orientation columns. Proc Natl Acad Sci U S A 83:8779-8783.

Livingstone M, Hubel D (1988) Segregation of form, color, movement, and depth: anatomy, physiology, and perception. Science 240:740-749.

Livingstone MS, Hubel DH (1983) Specificity of cortico-cortical connections in monkey visual system. Nature 304:531-534.

Livingstone MS, Hubel DH (1984a) Specificity of intrinsic connections in primate primary visual cortex. J Neurosci 4:2830-2835.

Livingstone MS, Hubel DH (1984b) Anatomy and physiology of a color system in the primate visual cortex. J Neurosci 4:309-356.

Livingstone MS, Hubel DH (1987) Psychophysical evidence for separate channels for the perception of form, color, movement, and depth. J Neurosci 7:3416-3468.

Lund JS, Yoshioka T, Levitt JB (1993) Comparison of intrinsic connectivity in different areas of macaque monkey cerebral cortex. Cereb Cortex 3:148-162.

Maguire WM, Meyer GE, Baizer JS (1980) The McCollough effect in rhesus monkey. Invest Ophthalmol Vis Sci 19:321-324.

Malach R, Tootell RB, Malonek D (1994) Relationship between orientation domains, cytochrome oxidase stripes, and intrinsic horizontal connections in squirrel monkey area V2. Cereb Cortex 4:151-165.

Malach R, Amir Y, Harel M, Grinvald A (1993) Relationship between intrinsic connections and functional architecture revealed by optical imaging and in vivo targeted biocytin injections in primate striate cortex. Proc Natl Acad Sci U S A 90:10469-10473. 
Marcus DS, Van Essen DC (2002) Scene segmentation and attention in primate cortical areas V1 and V2. J Neurophysiol 88:2648-2658.

Marks WB, Dobelle WH, Macnichol EF, Jr. (1964) Visual Pigments of Single Primate Cones. Science 143:1181-1183.

Masland RH (1986) The functional architecture of the retina. Sci Am 255:102-111.

Maunsell JH, Gibson JR (1992) Visual response latencies in striate cortex of the macaque monkey. J Neurophysiol 68:1332-1344.

Mazer JA, Vinje WE, McDermott J, Schiller PH, Gallant JL (2002) Spatial frequency and orientation tuning dynamics in area V1. Proc Natl Acad Sci U S A 99:1645-1650.

Merigan WH, Maunsell JH (1993) How parallel are the primate visual pathways? Annu Rev Neurosci 16:369-402.

Miki N, Keirns JJ, Marcus FR, Freeman J, Bitensky MW (1973) Regulation of cyclic nucleotide concentrations in photoreceptors: an ATP-dependent stimulation of cyclic nucleotide phosphodiesterase by light. Proc Natl Acad Sci U S A 70:3820-3824.

Miller KD, Keller JB, Stryker MP (1989) Ocular dominance column development: analysis and simulation. Science 245:605-615.

Mitchison G (1991) Neuronal branching patterns and the economy of cortical wiring. Proc Biol Sci 245:151-158.

Mitchison G (1995) A type of duality between self-organizing maps and minimal wiring. Neural Comput 7:25-35.

Munk MH, Nowak LG, Girard P, Chounlamountri N, Bullier J (1995) Visual latencies in cytochrome oxidase bands of macaque area V2. Proc Natl Acad Sci U S A 92:988-992.

Nowak LG, Bullier J (1998) The timing of information transfer in the visual system. In: Cerebral Cortex (Kaas JH, Rockland K, Peters A, eds), pp 205-241. New York: Plenum Press.

Nowak LG, Munk MH, Girard P, Bullier J (1995) Visual latencies in areas V1 and V2 of the macaque monkey. Vis Neurosci 12:371-384.

O'Brien DF (1982) The chemistry of vision. Science 218:961-966.

Olavarria JF, Van Essen DC (1997) The global pattern of cytochrome oxidase stripes in visual area V2 of the macaque monkey. Cereb Cortex 7:395-404.

Olshausen BA, Field DJ (1997) Sparse coding with an overcomplete basis set: a strategy employed by V1? Vision Res 37:3311-3325. 
Oram MW, Xiao D, Dritschel B, Payne KR (2002) The temporal resolution of neural codes: does response latency have a unique role? Philos Trans R Soc Lond B Biol Sci 357:987-1001.

Pei YF, Rhodin JA (1970) The prenatal development of the mouse eye. Anat Rec 168:105-125.

Piepenbrock C, Obermayer K (2000) The effect of intracortical competition on the formation of topographic maps in models of Hebbian learning. Biol Cybern 82:345-353.

Priebe NJ, Lisberger SG, Movshon JA (2006) Tuning for spatiotemporal frequency and speed in directionally selective neurons of macaque striate cortex. J Neurosci 26:2941-2950.

Raiguel SE, Lagae L, Gulyas B, Orban GA (1989) Response latencies of visual cells in macaque areas V1, V2 and V5. Brain Res 493:155-159.

Roe AW, Ts'o DY (1995) Visual topography in primate V2: multiple representation across functional stripes. J Neurosci 15:3689-3715.

Roe AW, Ts'o DY (1999) Specificity of color connectivity between primate V1 and V2. J Neurophysiol 82:2719-2730.

Roe AW, Fritsches K, Pettigrew JD (2005) Optical imaging of functional organization of V1 and V2 in marmoset visual cortex. Anat Rec A Discov Mol Cell Evol Biol 287:1213-1225.

Lu HD, Roe AW (2008) Functional organization of color domains in V1 and V2 of macaque monkey revealed by optical imaging. Cereb Cortex 18:516-533.

Rosa MG (2002) Visual maps in the adult primate cerebral cortex: some implications for brain development and evolution. Braz J Med Biol Res 35:1485-1498.

Sawatari A, Callaway EM (1996) Convergence of magno- and parvocellular pathways in layer 4B of macaque primary visual cortex. Nature 380:442-446.

Schein SJ, de Monasterio FM (1987) Mapping of retinal and geniculate neurons onto striate cortex of macaque. J Neurosci 7:996-1009.

Schmolesky MT, Wang Y, Hanes DP, Thompson KG, Leutgeb S, Schall JD, Leventhal AG (1998) Signal timing across the macaque visual system. J Neurophysiol 79:3272-3278.

Shapley R, Lennie P (1985) Spatial frequency analysis in the visual system. Annu Rev Neurosci 8:547-583.

Shipp S, Zeki S (2002) The functional organization of area V2, I: specialization across stripes and layers. Vis Neurosci 19:187-210.

Sholl DA (1953) The Organization of the Cerebral Cortex. London: Methuen and Co. 
Sincich LC, Horton JC (2002) Divided by cytochrome oxidase: a map of the projections from V1 to V2 in macaques. Science 295:1734-1737.

Sincich LC, Horton JC (2003) Independent projection streams from macaque striate cortex to the second visual area and middle temporal area. J Neurosci 23:5684-5692.

Sincich LC, Horton JC (2005) The circuitry of V1 and V2: integration of color, form, and motion. Annu Rev Neurosci 28:303-326.

Sincich LC, Park KF, Wohlgemuth MJ, Horton JC (2004) Bypassing V1: a direct geniculate input to area MT. Nat Neurosci 7:1123-1128.

Smith MA, Bair W, Movshon JA (2006) Dynamics of suppression in macaque primary visual cortex. J Neurosci 26:4826-4834.

Spiridon M, Gerstner W (2001) Effect of lateral connections on the accuracy of the population code for a network of spiking neurons. Network 12:409-421.

Stemmler M, Usher M, Niebur E (1995) Lateral interactions in primary visual cortex: a model bridging physiology and psychophysics. Science 269:1877-1880.

Stepanyants A, Chklovskii DB (2005) Neurogeometry and potential synaptic connectivity. Trends Neurosci 28:387-394.

Stone J, Leicester J, Sherman SM (1973) The naso-temporal division of the monkey's retina. J Comp Neurol 150:333-348.

Swindale NV (2007) A model for the thick, thin and pale stripe organization of primate V2. Network 18:327-342.

Swindale NV, Shoham D, Grinvald A, Bonhoeffer T, Hubener M (2000) Visual cortex maps are optimized for uniform coverage. Nat Neurosci 3:822-826.

Szeliski R (1990) Bayesian modeling of uncertainty in low-level vision International Journal of Computer Vision 5:271-301.

Tamura H, Sato H, Katsuyama N, Hata Y, Tsumoto T (1996) Less segregated processing of visual information in V2 than in V1 of the monkey visual cortex. Eur J Neurosci 8:300309.

Tootell RB, Hamilton SL (1989) Functional anatomy of the second visual area (V2) in the macaque. J Neurosci 9:2620-2644.

Tootell RB, Silverman MS, Hamilton SL, De Valois RL, Switkes E (1988) Functional anatomy of macaque striate cortex. III. Color. J Neurosci 8:1569-1593. 
Ts'o DY, Gilbert CD (1988) The organization of chromatic and spatial interactions in the primate striate cortex. J Neurosci 8:1712-1727.

Ts'o DY, Roe AW, Gilbert CD (2001) A hierarchy of the functional organization for color, form and disparity in primate visual area V2. Vision Res 41:1333-1349.

Van Essen DC, Newsome WT, Maunsell JH, Bixby JL (1986) The projections from striate cortex (V1) to areas V2 and V3 in the macaque monkey: asymmetries, areal boundaries, and patchy connections. J Comp Neurol 244:451-480.

Vanduffel W, Tootell RB, Schoups AA, Orban GA (2002) The organization of orientation selectivity throughout macaque visual cortex. Cereb Cortex 12:647-662.

VanRullen R, Guyonneau R, Thorpe SJ (2005) Spike times make sense. Trends Neurosci 28:1-4.

Wang Y, Xiao Y, Felleman DJ (2006) V2 Thin Stripes Contain Spatially Organized Representations of Achromatic Luminance Change. Cereb Cortex.

Watts RW, Marley J, Worley P (1998) Undergraduate education in anaesthesia: the influence of role models on skills learnt and career choice. Anaesth Intensive Care 26:201-203.

Wiesel TN, Hubel DH (1966) Spatial and chromatic interactions in the lateral geniculate body of the rhesus monkey. J Neurophysiol 29:1115-1156.

Wong-Riley M (1979) Changes in the visual system of monocularly sutured or enucleated cats demonstrable with cytochrome oxidase histochemistry. Brain Res 171:11-28.

Wong-Riley MT (1989) Cytochrome oxidase: an endogenous metabolic marker for neuronal activity. Trends Neurosci 12:94-101.

Xiao Y, Felleman DJ (2004) Projections from primary visual cortex to cytochrome oxidase thin stripes and interstripes of macaque visual area 2. Proc Natl Acad Sci U S A 101:71477151.

Xiao Y, Wang Y, Felleman DJ (2003) A spatially organized representation of colour in macaque cortical area V2. Nature 421:535-539.

Yabuta NH, Callaway EM (1998) Functional streams and local connections of layer 4C neurons in primary visual cortex of the macaque monkey. J Neurosci 18:9489-9499.

Yamada E, Ishikawa T (1965) The fine structure of the horizontal cells in some vertebrate retinae. Cold Spring Harb Symp Quant Biol 30:383-392.

Yoshioka T, Dow BM (1996) Color, orientation and cytochrome oxidase reactivity in areas V1, V2 and V4 of macaque monkey visual cortex. Behav Brain Res 76:71-88. 
Yoshioka T, Blasdel GG, Levitt JB, Lund JS (1996) Relation between patterns of intrinsic lateral connectivity, ocular dominance, and cytochrome oxidase-reactive regions in macaque monkey striate cortex. Cereb Cortex 6:297-310.

Zeki S, Watson JD, Lueck CJ, Friston KJ, Kennard C, Frackowiak RS (1991) A direct demonstration of functional specialization in human visual cortex. J Neurosci 11:641649.

Zohary E, Shadlen MN, Newsome WT (1994) Correlated neuronal discharge rate and its implications for psychophysical performance. Nature 370:140-143. 
CHAPTER 2: Objectives and Rationale 
This investigation utilized multiple techniques to better observe and hypothesize on the role of clustered organization and the generation of mixed properties in V2. The first set of experiments used network modeling to examine the interaction of cell groupings (Chapter 3) while the second set of experiments employed electrophysiology to further characterize the changes in neuronal latencies in V2 of anesthetized macaque monkey (Chapter 4). The experiments presented here were designed to investigate two separate objectives; the results of these experiments are presented in Chapters 3 and 4. This chapter outlines the objectives and rationale for these experiments.

\subsection{Objective 1 - Develop a biologically inspired computational network model to investigate and characterize the relationship between clustered functional organization and probability of convergent combinatorial circuitry within Area V2}

As described in Chapter 1, the clustered functional organization of V2 could provide a framework for significant and comprehensive convergence of neuronal terminals from neighboring orientation-preferring and color-preferring neuronal populations. Computer based modeling allows the implementation and controlled manipulation of anatomically based cortexlike networks. These networks can be configured to plausibly represent clustered functional configurations known to occur in V2. Key parameters such as network size, model analogs of axon/dendrite length and terminal bouton density, and the balance of intra- vs. inter-areal inputs may be specified under guidance of published data. Importantly, modeling also allows for the specification of distinct spatial organizations of response properties. Model cells can be assigned specific (and distinct) tuning properties, such as preferences for particular stimulus orientations or colors and clusters of like-preferring model cells can be arranged to investigate the probability of combinatorial circuitry between the groups. Thus, modeling provides a flexible and highly controlled framework for the investigation of the possible functional roles of clustered organization in cortex (in general), and the investigation of the possible generation of mixed properties in V2 in particular.

To investigate the potential for preference integration and cluster roles, this dissertation presents two probabilistic models with organizational features based on neighboring domain organization found in the macaque V2. The formulation and results of these models are detailed in Chapter 3. Both models consisted of two-dimensional spatial arrays representing the 
functional organizations of small regions of the cortical topographic map in V2. The first model was devised to investigate how local circuitry distribution and preference characteristics might support functional integration within and between a pair of neighboring yet differently preferring neuron clusters, devised to investigate the role of domains in V2 and the probability of neurons with multiple preferences. The second model involved a much larger number of domains than the first as it was devised to investigate if sufficiently diverse domain types fell within a reasonable proximity to permit an expectedly broad range of possible integration combinations.

These modeling experiments were primarily performed to further elucidate possible computational/functional benefits associated with specific spatial organizations (i.e. clustering) in cortex. However the model experiments and their findings may also be applied to other aspects of cortex-like network function in general. For example, with the recent rise of biologically-inspired computing, it may be important to understand the relative computational benefits of specifically distributed clusters of computational elements. The future development of brain/machine interfaces (via monitoring of specific cortical domains) and artificial prostheses for the visually impaired (via stimulation of specific cortical domains) may also benefit by better understanding of the role and function of clustered organizations in the cortex.

\subsection{Objective 2 - Compare and quantify the differential temporal responses associated with achromatic-oriented vs. color-oriented stimuli in Area V2 neurons}

As described in Chapter 1, the temporal response properties of a V2 neuron associated with specific color and orientation stimulation have been characterized to some degree but are still not well understood. Further characterizations of these responses would be advantageous for several reasons. Identification of differential response patterns could be correlated with other organizational aspects of cortex, and thus provide insight into the functional roles of specific organizations of cortical neurons in V2. For instance, parameters such as latency can suggest probable pathways of visual information: changes in a particular cell's latency by modulating the stimulus modality (e.g. color vs. orientation) can suggest differing processing inputs to a common unit. This information can shed light on how cells of common preference type cluster together and the circuitry leading up to cells possessing multiple preference types. Furthermore, 
these characterizations can provide useful base data on which new experiments can be subsequently proposed and implemented.

Chapter 4 presents single unit electrophysiological recordings from macaque monkey V2 (and their associated analyses) following controlled colored- and/or achromatic-oriented visual stimulations. Following intrinsic signal imaging of the cortical surface (to delineate V2 functional cortex), the latency and temporal response characteristics of single neuron responses were quantified and statistically compared. Modulations in temporal properties can indicate possible circuitry utilized with differing visual stimuli, such as the potential intra-areal interaction between color and orientation functional domains. Additionally, these temporal relationships further increase understanding of the spiking response visual neurons produce in response to elementary feature stimuli. Together, the experiments detailed in Chapter 4 intend to characterize and quantify new details of the temporal changes spiking activity of orientationpreferring cells in macaque V2 in response to achromatic oriented vs. color oriented visual stimulation. 
CHAPTER 3: Clustered cortical organization and the enhanced probability of intra-areal functional integration 


\subsection{Abstract}

Similarly responsive neurons organize into submillimeter-sized clusters (domains) across many neocortical areas, notably in Areas V1 and V2 of primate visual cortex. While this clustered organization may arise from wiring minimization or from self-organizing development, it could potentially support important neural computation benefits. Here, we suggest that domain organization offers an efficient computational mechanism for intra-areal functional integration in certain cortical areas and hypothesize that domain proximity could support a higher-thanexpected spatial correlation of their respective terminals yielding higher probabilities of integration of differing domain preferences. To investigate this hypothesis we devised a spatial model inspired by known parameters of V2 functional organization, where neighboring domains prefer either colored or oriented stimuli. Preference-selective joint probabilities were calculated for model terminal co-occurrence with configurations encompassing diverse domain proximity, shape, and projection. Compared to random distributions, paired neighboring domains $(\leq$ $1200 \mu \mathrm{m}$ apart) yielded significantly enhanced coincidence of terminals converging from each domain. Using this reference data, a second larger-scale model indicated that V2 domain organization may accommodate relatively complete sets of intra-areal color/orientation integrations. Together, these data indicate that domain organization could support significant and efficient intra-areal integration of different preferences and suggest further experiments investigating prevalence and mechanisms of domain-mediated intra-areal integration. 


\subsection{Introduction and Background}

A predominant feature of primate cortical organization is the tendency for neurons with similar response properties to be arranged within close spatial proximity. This organizational principle is well documented via electrophysiological studies (Mountcastle et al., 1957; Hubel and Wiesel, 1969), anatomical appraisals (Szentagothai, 1978; Peters and Sethares, 1996) and high resolution functional imaging methods (Grinvald et al., 1986). Together these methods have now identified prevalent neuronal groupings ('functional domains') with a higher probability of shared single neuron response characteristics and stereotypical topographic organizations, especially in sensory cortex of the cat, human and non-human primate (Kim et al., 2000; Ramsden et al., 2001; Ohki et al., 2005; Chen et al., 2007; Yacoub et al., 2008). However, the extent that a functional domain organization might explicitly contribute to specific neuronal computation has been recently debated and remains unclear (Horton and Adams, 2005). For example, functional domains may simply be a consequence of an efficient way to 'wire up' the brain (Koulakov and Chklovskii, 2001). Intuitively, however, clustered organizations might offer some special intra-areally mediated integrative advantages for cortical neurons: first, by spatially constraining the available classes of local terminal input (supporting a more selective integration), and secondly, by increasing selective terminal input densities (supporting a stronger integrative signal above noise). The likelihood of such an intra-areal mechanism would depend on the statistics of terminal co-distributions associated with neurons mapped to particular domains. Figure 3-1A, for example, shows a simplified idealized cortical representation where a limited portion (grey shaded area) of randomly distributed terminals (circles) with different stimulus preference properties (circle shadings) is sampled by a secondary integrative neuron. When there are only two terminal preference classes available, a clustered organization (Figure 3-1A, top left) offers at best the same overall number and density of 'black' and 'white' terminal pairings as a mixed spatial organization (Figure 3-1A, top right). However when the possible terminal classes increase from two to four, a clustered organization allows the same neuron to sample a more selective subset of terminals. The local probability of co-occurrence of 'black' and 'white' class terminals is now higher with clustered organization (Figure 3-1A, bottom left) than with mixed organization (Figure 3-1A, bottom right), and importantly the sampling secondary neuron can now efficiently respond to a combination of the two stimulus attributes. 


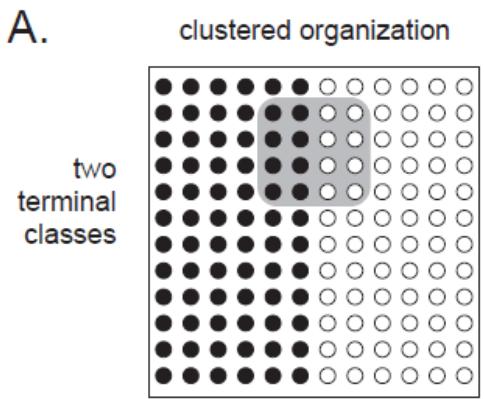

$n_{0} \times n_{0}=64$

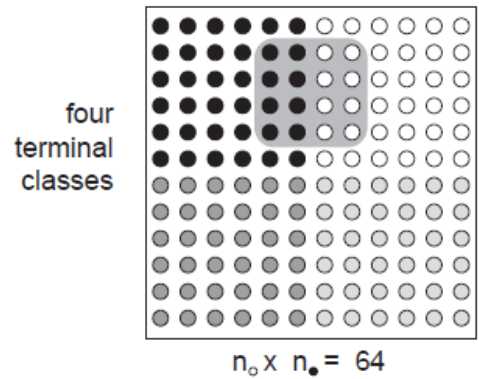

mixed organization

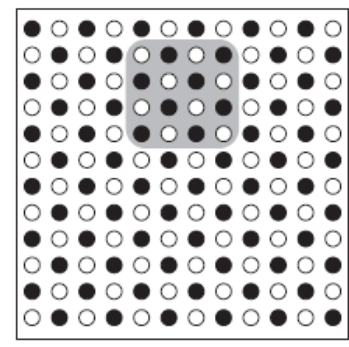

$n_{0} \times n_{\bullet}=64$

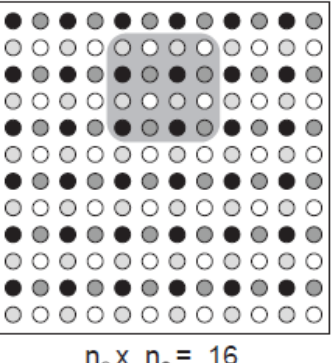

B. visual neurons

grouped by

orientation preference color preference

Area V2

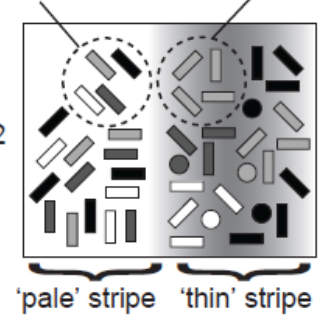

C.

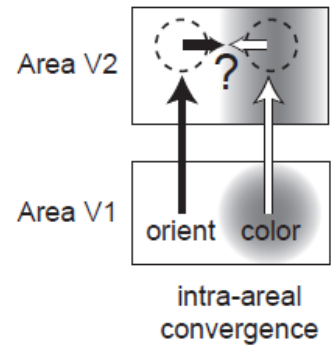

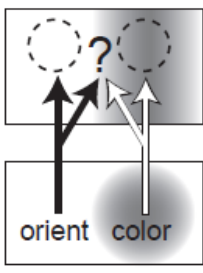

inter-areal convergence

Figure 3-1: Clustered cortical organization: an efficient mechanism for potent intra-areal integration of neuronal inputs with different stimulus preferences? Clustered functional organizations may offer special integrative advantages for cortical neurons by spatially constraining available classes of terminal input, and thereby increasing their local density and elevating the probability of their local co-occurrence. For example, in an idealized cortical representation, (A), a limited portion (grey shaded area) of randomly distributed terminals (circles) with different stimulus preference properties (circle shadings) is sampled by a secondary integrative neuron. The secondary neuron samples a higher density of black and white class terminal pairings ( $A$, bottom left) than with mixed organization ( $A$, bottom right), and importantly can now respond optimally to a combination of the two stimulus attributes. This differential advantage may increase as the cluster organization becomes more diverse (compare top row, two classes, with bottom row, four classes). The second visual cortical area in primates, Area V2, (B) could take advantage of such a cluster-enhanced integrative intra-areal mechanism. Visually responsive V2 neurons are distinctly clustered according to preference for stimulus orientation (in histologically defined 'pale' stripes) vs. color (in adjacent 'thin' stripes), an organization which could support increased probability of color and orientation terminal co-occurrence and subsequent color/orientation integration. A functional implementation will however critically depend on cell and terminal distribution geometry, sampling locations, and diversity of grouped stimulus attributes in the color and orientation map. Additionally, for this mechanism to allow complete combinations, a sufficient complement of preference pairings should be within a feasible integrative reach. Our modeling investigates and quantifies the probabilities of cluster-mediated intra-areal integration of color and orientation attributes $(C$, left) as a feasible and potent means to support other previously proposed mechanisms such as inter-areally mediated convergence (C, right).

One area that could readily take advantage of such a putative cluster-enhanced integrative intra-areal mechanism is the second visual cortical area in primates, Area V2. The functional organization of this area is very well documented (Tootell and Hamilton, 1989; Roe and Ts'o, 1995; Xiao et al., 1999; Ts'o et al., 2001; Vanduffel et al., 2002; Xiao et al., 2003; Wang et al., 2007; Lu and Roe, 2008; Ts'o et al., 2009), indicating that visually responsive neurons in V2 are distinctly clustered (Figure 3-1B) according to preferences for stimulus orientation (in histologically defined 'pale' stripes) and color (in adjacent 'thin' stripes). This organization 
locates relatively large neuronal domains (typically about $250-1000 \mu \mathrm{m}$ in breadth) comprising differing functional preferences (color vs. orientation) in adjacent stripe compartments, and therefore within proximity of about $1 \mathrm{~mm}$ or less. While the larger scale functional organization of V2 is well understood, the role of this organization in shaping individual neuronal response properties is not well understood. We suggest that a proximity of preference domains could form the basis for increased probability of color- and orientation-preferring terminal co-occurrence and consequent intra-areally mediated color/orientation integration. A practical functional implementation of such a mechanism will, however, critically depend on terminal distribution geometry, sampling locations, and diversity of grouped stimulus attributes across the color and orientation map. Additionally, for this mechanism to allow a sufficiently complete representation of all combinations (coverage), all (or at least a very high proportion) of possible pairings should be within a feasible integrative reach.

With the recent emergence of neuroinformatics and biologically-inspired computing design, there is a need for improved knowledge of the relative roles of inter-areal vs. intra-areal cortical circuitry and of the comparative functions of clustered vs. distributed organizations in cortical computation. Improved understanding of these principles would also guide neurobiological experimental design and data interpretation and expand our understanding of the cortex as a complex integrative functional system. How neuronal preferences for different stimulus attributes are combined in V2, in particular, is also a fundamentally important question to investigate. It has been suggested that V2 is involved in more mixed or integrative computations compared with the primary visual cortex, Area V1 (Tamura et al, 1996) but the relative roles of intra-areal vs. inter-areal mechanisms in V2 are unclear. There is also evidence of distinct color-dominant inter-areal projection pathways (V1 blob regions to V2 thin stripes) in parallel with orientation-dominant pathways (V1 inter-blob regions to V2 pale stripes), thus paving a potential for color/orientation integration intra-areally at the level of V2 (Sincich and Horton, 2002)

Here we use known V2 organization principles and constraints to design probabilistic intra-areal spatial models of terminal preference and co-distribution. We use these spatial models to test our first hypothesis: that functional domain organization in V2 significantly increases the probability that local terminal distributions will share combined preference properties of the nearby domains. In particular, we quantify the probabilities of cluster-mediated intra-areal 
integration of color and orientation attributes through common input projection (Figure 3-1C, left). We also implement a second model based on known V2 organization that examines the potential for complete representation of preference combinations. Again, we focus on the likelihood of integration of projections from color and orientation domains motivated by our second hypothesis: that V2 organization could provide the necessary geometry for a sufficiently complete coverage of color/orientation integration. Supported by the results obtained from Model 1 our data obtained from Model 2 suggest that functional clustering could potentially provide a significant organizational advantage for the new development, or further consolidation, of mixed sensory properties in V2, and we suggest that this could supplement other previously proposed mechanisms such as inter-areally mediated convergence (Figure 3-1C, right) (Hubel and Wiesel, 1962; Felleman and Van Essen, 1991). We suggest that these principles may also apply to integrate other domain classes in V2 and in other cortical areas with clustered functional organizations. 


\subsection{Model Frameworks as Representations of Functional Domain Organization in Cortex}

To investigate potential for functional integration via terminal projections to common targets from neighboring functional domains within V2, we devised two model frameworks (Model 1 and Model 2). These exhibited simplified but analogous fundamental topographic features to those reported in V2 (MatLab, The Mathworks, Natick, MA). Because we were primarily interested in the influence that domain organization may have on functional integration in V2, we purposely designed model frameworks that would allow us to manipulate spatial functional organization and its associated circuitry in a highly controlled manner. Thus while the models did not incorporate detailed realistic circuitry features, they nevertheless incorporated essential elementary organizational features that we felt most appropriate for our aims (e.g., domain shape and breadth, preference characteristics, systematic extent of model axonal and dendritic arbors). Local ('intrinsic') intra-cortical circuitry comprises two essential distributions in Area V2: a radially diffuse distribution of axonal projections that exists in the immediate vicinity of the neurons, and a more heterogeneous patchy distribution that are known to extend up to several millimeters from a neuron (Amir et al., 1993; Lund et al., 1993). In these initial computational experiments, we chose to primarily specify radially diffuse rather than also introduce patchy terminal distributions in our models because the precise details of the patchy projection mappings are still not sufficiently documented in V2. We also felt a focus on radially diffuse processes would initially allow a simpler model specification at the outset of these studies, and that this could provide a more conservative estimate of joint terminal occurrence as a function of distance between various neuronal sources (since we presumed that any additional specific patchy or other heterogeneous projections would likely further enhance joint terminal occurrence to some finite degree). As an additional control experiment however we also investigated the introduction of patchy distributions to our model (see Figure 3-9). Here we were motivated to investigate the general differential effect of radially diffuse vs. patchy projection patterns on joint terminal occurrence when all other parameters, including total terminals projected, are unchanged. We also did not elect to explicitly model spatio-temporal integration within these model cells. Rather, we specifically focused on the local spatial co-distributions of terminals across the array for different circuitry configurations and different functional preferences. In particular, we tracked the relative spatial co-distributions of functional preference 
of terminals to quantify when some organizational configurations might lead to more functionally heterogeneous connections than otherwise expected.

Both model frameworks consisted of two-dimensional spatial arrays that represented small regions of the cortical topographic map in V2. Model 1 was devised to investigate how local circuitry distribution and preference characteristics might support functional integration within and between a pair of neighboring yet differently selective functional domains. We reasoned that if integration of the properties associated with the paired domains could occur within an area, then we would expect to see instances of significantly greater spatial cooccurrence of specific terminal classes than would be found in randomly generated (domain-less) organizations. Our design of a reduced single and dual domain configuration (Model 1) allowed for a strictly controlled investigation of specific spatial relationships without the interpretative confounds associated with a larger (biologically analogous) multiple domain set. Importantly, these data and analyses obtained from Model 1 allowed us to draw conclusions from data obtained in our second model, Model 2. Model 2 involved a much larger number of domains than Model 1 and it was devised to investigate if sufficiently diverse domain types fell within a reasonable proximity to permit an expectedly broad range of possible integration combinations. We reasoned that if intra-areal circuits are an important cortical mechanism for functional integration, then these circuits should support functional integrations that are diverse and sufficiently complete. 


\subsection{Model 1 Methods: A Topographical Framework to Investigate Functional Integration}

\section{Associated with Domain Pairs}

\subsubsection{Two-Dimensional Representation of Neuronal Terminal Distributions}

Model 1 consisted of a two-dimensional spatial array of model 'cells' that represented a spatial organization of neurons across a small region of the V2 functional map. In most cases this array comprised 100 x 100 cells in a single layer, spanning $2 \mathrm{~mm} \times 2 \mathrm{~mm}$ of analogous cortical space with cell-cell spacing at $20 \mu \mathrm{m}$ (Figure 3-2A). Each model cell represented a single neuron or small group of neurons at specific locations across the topographic cortical map. Because we were primarily interested in the probability of functional combination rather than other network phenomenon, we defined our cells as simple elemental nodes that projected output 'terminals' with designated stimulus preference characteristics. Thus our model calculated the relative codistributions of functional preference of all output elements across the array in systematically varying geometric and organizational scenarios.
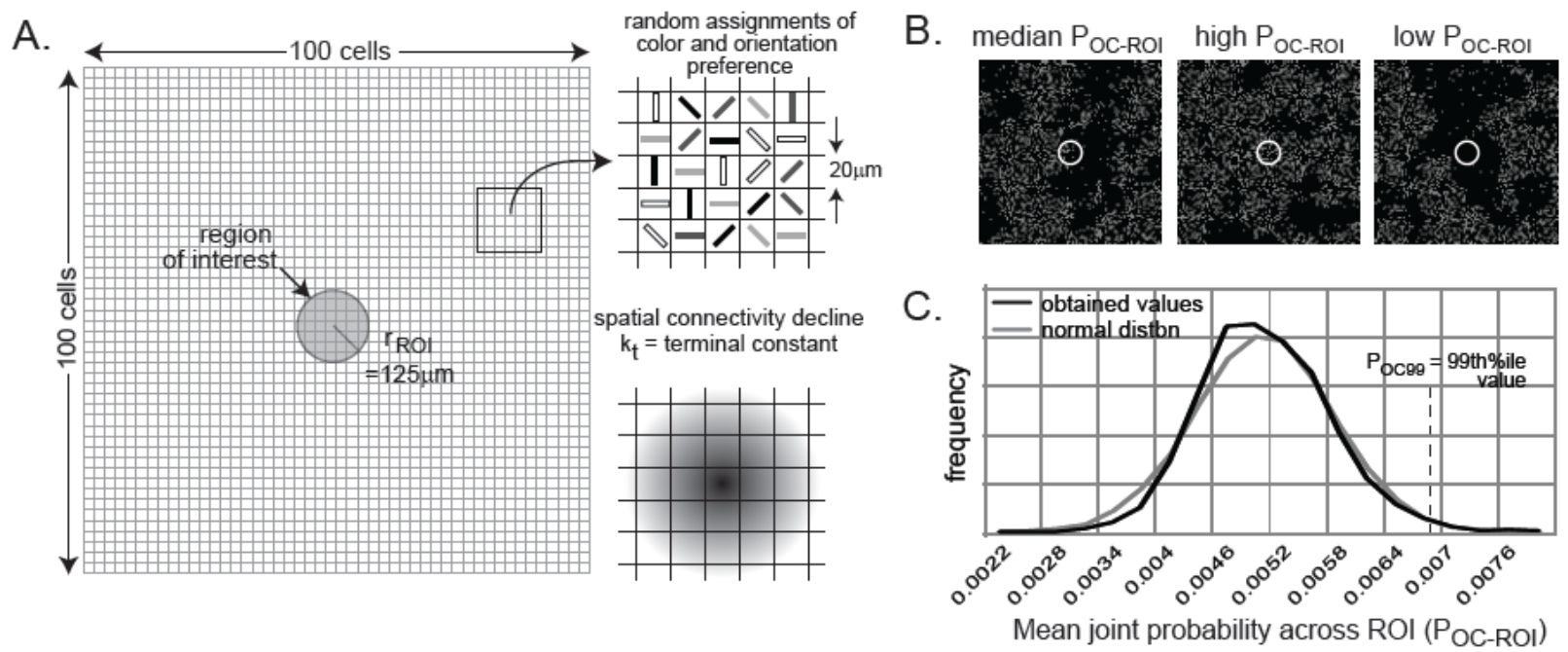

Figure 3-2. Model overview and joint probabilities in random (no domain) preference organization. (A) A grid array of cells $(100 \times 100$, each $20 \mu \mathrm{m} \times 20 \mu \mathrm{m})$ were randomly assigned one of eight orientation preferences and one of eight color preferences. Every cell distributed functional preference to neighboring cells via output 'terminals' of exponentially declining density (spatial decay constant, $\mathrm{kt}=100 \mu \mathrm{m}$ ). (B) Joint probability for colocation of terminals with a specific orientation and specific color preference (POC) was calculated at each location, and plotted as 2D- thresholded intensity maps. Black pixels, POC < mean+3SD of POC for all randomly assigned array values. Grey pixels, POC $>$ mean+3SD of POC for all random array values. See Figure 3-6A for color coded POC maps. Note the cloudy random organizations of elevated POC. A circular region of interest (ROI, radius $125 \mu \mathrm{m}$ ) was centrally placed (superimposed circles, B) and the average POC, (POC-ROI), was calculated for cell locations within the ROI. (C) POC-ROI values obtained for 2500 randomizations (normally distributed, mean $=0.0049$, sd $=0.0007)$. The 99th percentile value $($ POC99 $=0.0069)$ defined an upper confidence limit reference for non-random configurations. 
We used published data where possible to guide our model design and parameter specifications (cortical biology: Amir et al.1993, Levitt, Kiper et al 1994, Levitt, Yoshioka et al 1994, Malach et al. 1994 , Roe and Ts’o 1995, Elston and Rosa 1997, Markram et al. 1997, Olavarria and Van Essen 1997, Ramsden et al. 2001, Ts’o et al. 2001, Stepanyants et al 2002, Angelucci et al 2002, Xiao et al 2003, Ohki et al 2005, Feldmeyer et al 2006, Lu and Roe 2008, Ts’o et al 2009; model networks: Sholl 1953, Abeles 1991,Stemmler et al 1995, Spiridon and Gerstener 2001, Kang et al 2003). Parameter choices are further discussed in the Discussion. For each cell, the spatial density of projecting terminals decreased radially and equally in all directions, defined by exponential spatial decay constants (Sholl, 1953; Abeles, 1991). While homogenous radial falloff of projection density is not precisely consistent with known anatomy, it allows efficient computation of projections associated with our model design, and this general approach has been used by others in cortex network models (Stemmler et al., 1995; Spiridon and Gerstner, 2001; Kang et al., 2003). Values chosen reduced terminal density to less than 50\%, $25 \%, 10 \%$ and $1 \%$ of maximum by $80 \mu \mathrm{m}, 140 \mu \mathrm{m}, 240 \mu \mathrm{m}$ and $460 \mu \mathrm{m}$ respectively, which is qualitatively consistent with the immediate projection surrounds of tracer studies in V2 (Amir et al., 1993). Since our model is only concerned with the relative spatial distributions of static terminals, and not spatio-temporal interactions, we excluded possible inhibitory or suppressive effects associated with local GABAergic circuitry, and learning or self-organizational effects.

\subsubsection{Two-Dimensional Representation of Functional Domain Organization}

Since we were particularly interested in understanding how clustered organizations of differently selective neurons might interact across V2, we defined two classes of model cell preference: 'color' or 'orientation'. To allow for representation of differential tuning within each class, eight distinct tuning preferences were defined per class (equivalent to eight different color preferences and eight different orientation preferences). We chose eight preference subtypes to provide a diversity of tuning options while remaining sufficiently categorical to allow practical analysis. Thus by defining contiguous areas of the array with pre-designated functional preference (class and tuning) in the model, it was possible to seed biologically plausible model representations of functional domains. Every cell distributed functional preference to neighboring cells via output 'terminals' of exponentially declining density (spatial decay constant, $\mathrm{k}_{\mathrm{t}}$ ). In addition to within-array projections (analogous to intra-areal connections), a 
proportion of all terminals ('input bias'), usually 10\%, were assigned to represent contributing inter-areal inputs that projected from V1 onto V2. The precise input bias is not known in V2, however detailed quantifications in cat visual cortex suggests that the proportion of projections onto cortical areas may be quite small relative to intra-areal projections (e.g. Binzegger et al 2004). These terminals were allocated the same preference characteristics as their destination locations in the array. Overall terminal densities were held constant, usually at 1200/cell. Thus for a $10 \%$ input bias at 1200 terminals/cell location, 120 terminals were directly allocated to a given array location (analogous to V1 contributions) while the balance of 1080 terminals were allocated from other cells from within the array (analogous to intra-areal contributions within V2). To ensure that our conclusions were not influenced by a highly selective combination of model parameters, model output results were also obtained with alternative parameter specifications of terminal density, input bias and domain purity (proportion of domain cells sharing the preference of the domain).

To represent color/orientation organization reported in primate V2 (Roe and Ts'o, 1995; Ts'o et al., 2001; Shipp and Zeki, 2002; Xiao and Felleman, 2004) model domains were defined as either uniformly color-preferring or orientation-preferring. Figure 3-3A shows an example of a single domain configuration in which a single contiguous circular array sub-region contains cells that prefer a common specific orientation (but have random color preferences). In most cases, however, we defined configurations with an additional sub-region where cells prefer a common specific color (but have random orientation preferences) (e.g., paired domain configuration, Figure 3-4A) and the distance between the color and orientation domains in a paired domain configuration was systematically varied to examine the resulting local cooccurrence of terminals preferring a given color and orientation at each array co-ordinate. Domains were defined to be circular regions of $500 \mu \mathrm{m}$ diameter for most modeling experiments (cf. (Ts'o et al., 2009)). However in additional control experiments, domain size and shape, and spatial decay constants were also varied. To eliminate possible artifactual probability losses at the array periphery due to insufficiency of intra-areal input, model array size always exceeded our spatial requirements. For example, array sizes of 150x150 were initialized, but only the central 100x100 locations were used in our calculations and analyses. Because terminal output density declined exponentially with a decay constant of 100 microns, this ensured that cells in the central 100x100 co-ordinates always received a full complement of intra-areal inputs. To 
ensure that any observed variations in preference distribution were associated only with concurrent changes in neighboring domain organization and proximity, all cells outside the defined domains were always allocated random color and orientation preferences.

A.

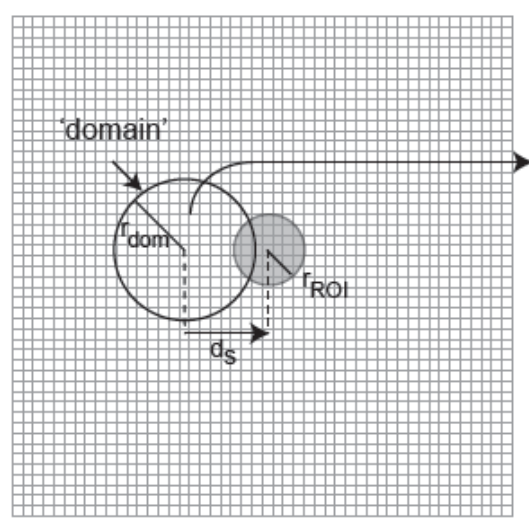

B.

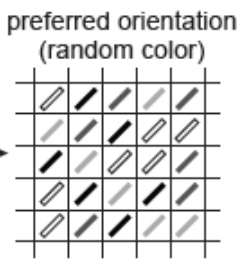

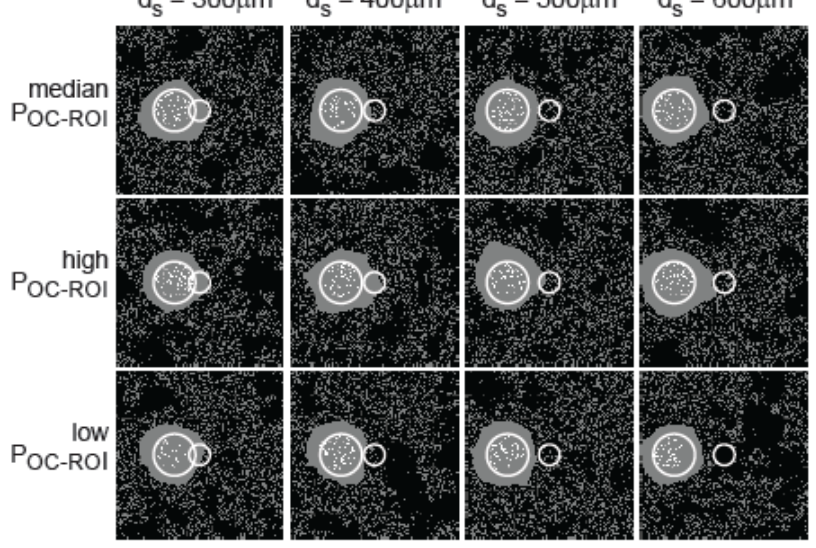

C.
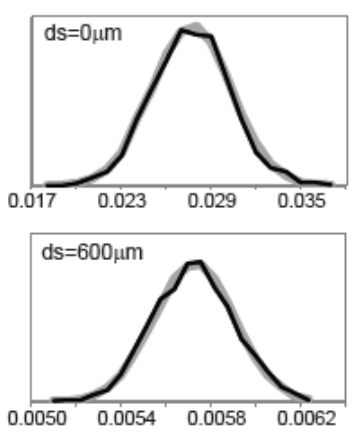

D.

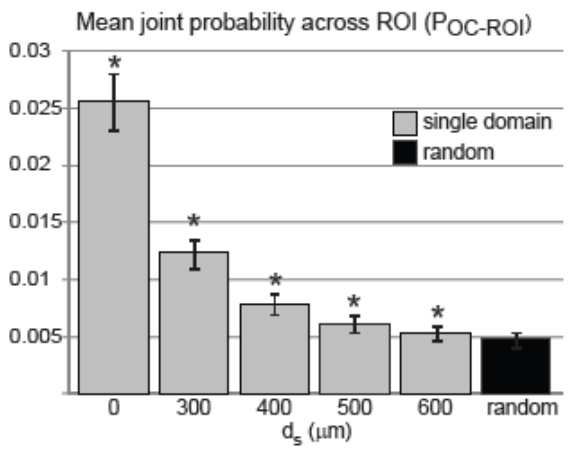

E.

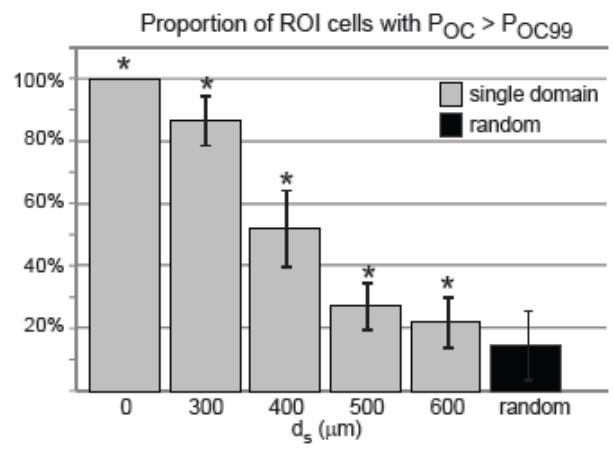

Figure 3-3. Domain organization significantly increases POC beyond the defined domain perimeter. (A) To investigate domain influences on neighboring regional POC magnitudes, cells within a circular region (rdom= $250 \mu \mathrm{m}$ ) were assigned one specific orientation preference and random color preference (analogous to a V2 orientation domain). All other cells were assigned random orientation and color preferences. The center of the domain was placed at varying distances (ds $=300,400,500$ and $600 \mu \mathrm{m}$ ) from the center of a circular ROI, and POC were determined for 100 randomizations in each case. (B) Examples of intensity-coded POC distributions for each ds. Domain perimeter, large white circle. ROI, small white circle. Black pixels: POC $<$ mean+3SD POC obtained from random only configuration, grey pixels: POC > mean+3SD POC obtained in random configuration, white pixels: POC > mean+3SD POC obtained in single domain configuration. Color coded examples are also shown in Fig. 3-6B. Notably, single domain organization introduced spatially extensive irregular shaped contiguous regions of highly elevated POC (grey areas in maps) that extended beyond the perimeter of the domain. (C) POC-ROI distributions at $d s=0 \mu \mathrm{m}$ and $\mathrm{ds}=600 \mu \mathrm{m}$ (black), superimposed on normal distributions (grey). (D) POC-ROI was highest when ROI was located at the domain center $(\mathrm{ds}=0)$, but POC-ROI for single domain configurations (light grey shaded bars) were significantly greater than for random configurations (black bars) when ROI and domain centers were separated by as much as $600 \mu \mathrm{m}$. (E) The proportion of ROI cells with POC $>$ POC99 also significantly increased up to $600 \mu \mathrm{m}$ from domain centers when compared to configurations without a domain. * = ANOVA $\mathrm{p}<0.0001$; Dunnett’s posthoc $\mathrm{p}<0.01$. Error bars $=+/-1$ SD. 
A.

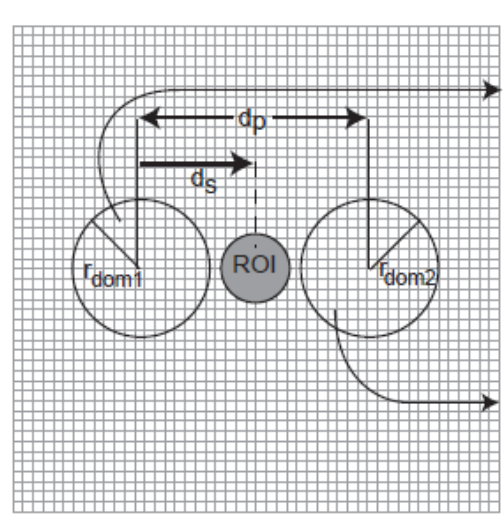

preferred orientation (random color)

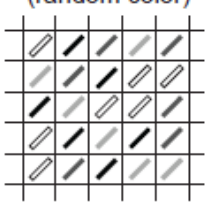

preferred color (random orientation)

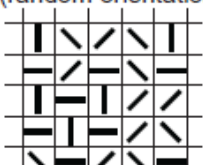

C.
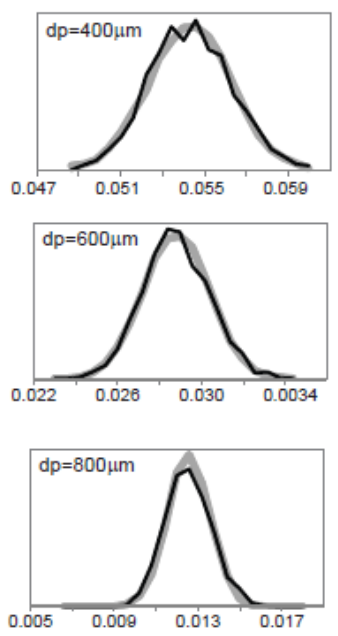

B.
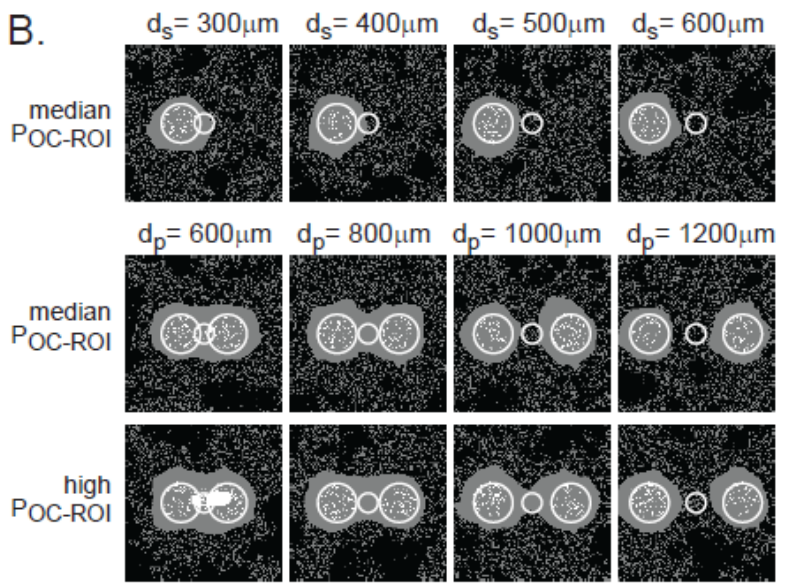

$d_{p}=1000 \mu m$

$d_{p}=1200 \mu \mathrm{m}$
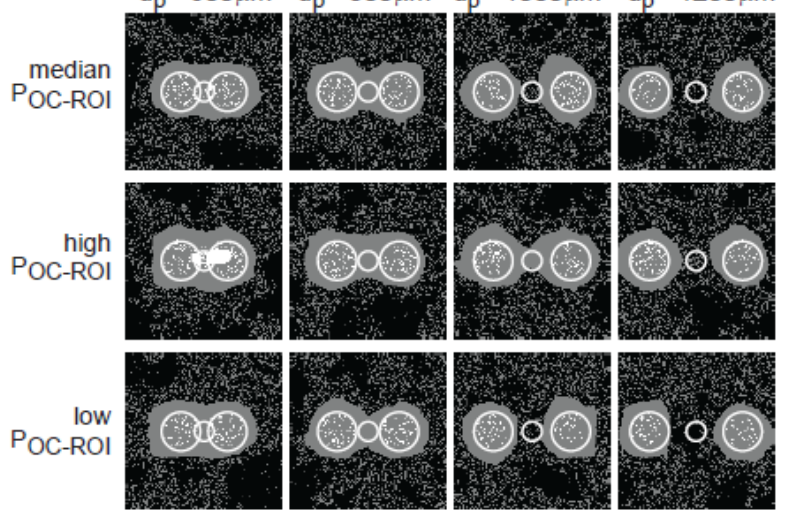

E.

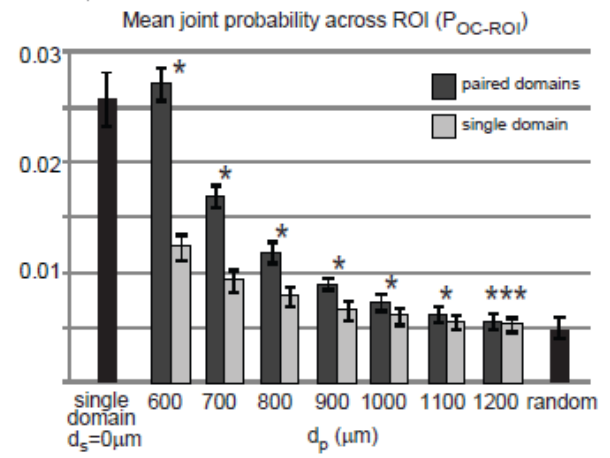

Figure 3-4. Paired domain configurations yield enhanced POC compared with single domain or random configurations. (A) Two model domains $(250 \mu \mathrm{m}$ radius) were placed at varying center-center distances ( $\mathrm{dp}=600$ $1200 \mu \mathrm{m}$ ), each equidistant from a central ROI. Note that $\mathrm{dp}=2 \mathrm{x}$ ds (ds is the comparable distance metric used in Fig. 3-3 for single domain maps). Left-placed domain cells were assigned common orientation preference and random color preference (analogous to V2 orientation domain). Conversely, the right-placed domain cells were assigned common color preference and random orientation preference (analogous to V2 color domain). All other cells were assigned random orientation and color preferences. (B) Examples of intensity-coded POC distributions for each dp, compared with single domain configurations at equivalent distances from ROI centers (ds). Map greyscale coding same as Fig.3-3. Color-coded examples are shown in Fig. 3-6C. Notably, paired domain organization introduced spatially extensive bridging regions of highly elevated POC (grey/white areas in maps). (C) POC-ROI distributions at $\mathrm{dp}=400,600$ and $800 \mu \mathrm{m}$ (black), superimposed on normal distributions (grey). (D) Paired domain configurations yielded significantly greater POC-ROI (dark grey boxes) than single domain configurations (light grey boxes) for all separations (dp) up to $1100 \mu \mathrm{m}$ (100 randomizations/configuration). Paired domains yielded approximately $120 \%, 80 \%$ and $50 \%$ enhancements of POC-ROI at 600,700 and $800 \mu \mathrm{m}$ respectively. Random configuration and single domain configuration $(\mathrm{ds}=0)$ data shown in black. Single domain data is repeated from Figure 3-3D, for reference. (E) Paired domain configurations yielded a higher proportion of ROI cell locations with POC $>$ POC99S than equivalently configured single domain configurations. Student's t-Test $*=\mathrm{p}<0.0001$, $* *=\mathrm{p}<0.01, * * *=\mathrm{p}<0.05$. Error bars $=+/-1 \mathrm{SD}$. 


\subsubsection{Mapping the Functional Joint Probability of Specific Color and Orientation Preference}

Common input is one mechanism that could lead to functional integration in V2. We reasoned that if more terminals of two different classes were co-localized at one array location compared with other locations, then a cell at that location would have a higher probability of receiving joint input from each terminal class. Thus, to assess the potential for functional integration between neighboring color-preferring and orientation-preferring model domains, we defined and calculated a functional joint probability $\left(\mathrm{P}_{\mathrm{OC}}\right.$ ), which quantified the likelihood of local common input. For example, the joint probabilities of terminals preferring one particular color and terminals preferring one particular orientation were calculated by multiplying their respective spatial densities at each location. Normalized functional joint probabilities were then obtained by subsequently scaling these products such that the sum of all possible joint probabilities across the array equated to unity. For our investigations, we arbitrarily selected the second orientation preference of eight possible orientations and the fourth color preference of eight possible color preferences for our uniform domains and for our subsequent detailed probability calculations. Since the preferences were defined to be equally probable overall, conclusions drawn from this precise selection can be generalized to other preference combinations. To ensure that calculations and interpretations were not biased by one particular random seed, 100 different random preference seedings were generated and joint probabilities recalculated for each configuration. Average joint probabilities across all iterations were then plotted as color-coded (e.g., Figures 3-6 and 3-7A) or grey scale intensity-coded twodimensional arrays (e.g., Figures 3-2B, 3-3B, 3-4B, and 3-9A). To investigate possible functional joint probability variation within selected sub-areas of the array, we defined a circular region of interest (ROI, 250 $\mu$ m diameter) which could be placed in predetermined locations within the array. In most cases the ROI was placed at the center of the array and average joint probabilities ( $\mathrm{P}_{\mathrm{OC}-\mathrm{ROI}}$ ) of $\mathrm{P}_{\mathrm{OC}}$ of all array co-ordinates in each $\mathrm{ROI}$ were calculated for each configuration. We initially ran 2500 randomizations to comprehensively investigate and confirm that obtained $\mathrm{P}_{\mathrm{OC}-}$ ROI exhibited a normal distribution for our various model configurations, but 100 randomizations were sufficient for subsequent experiments when detailed distributions did not need to be characterized. We then compared the obtained probabilities in response to different domain configurations. Two dimensional image representations of array $\mathrm{P}_{\mathrm{OC}}$ data and domain packing results were produced and averaged using MatLab (The Mathworks, Natick, MA). For statistical 
analysis, Student's t-tests were performed using Excel 2007 Analysis Tool Pak (Microsoft, USA) and Analyse-it v2.20 (Analyse-it Software Ltd, Leeds, UK) was used for all one way ANOVA and post-hoc analyses. All post-hoc comparisons were determined by Tukey's or Dunnett's method using a 99\% confidence interval.

\subsubsection{Controlled Manipulations of Model Domain Configurations and Associated Parameters}

By comparing the functional joint probabilities of specific color and orientation preferring terminals across our model array, we determined when the presence and proximity of functional domains may provide an efficient spatial distribution mechanism for intra-areal functional integration. Typically, two domains were defined in order to perform a strictly controlled analysis of terminal co-occurrence between a single neighboring domain pair at a range of center-center separations $\left(d_{P}\right)$ (Configuration C), (e.g., Figure 3-4A). Further controlled manipulation of parameters relating to within-array connectivity then allowed the calculation of joint probabilities, $\mathrm{P}_{\mathrm{OC}}$, across a number of configuration scenarios. In particular, we compared $\mathrm{P}_{\mathrm{OC}}$ obtained in paired domain configurations with those obtained in several control configurations. One control configuration (Configuration A) had no domain organization, i.e., the cells were randomly assigned a color and orientation preference (e.g., Figure 3-2A). A second control configuration (Configuration B) had only one domain defined, used to provide probability control data in the absence of a second paired domain (e.g., Figure 3-3A). Configuration $\mathrm{D}$ investigated the relationship between spatial distributions of joint probability and elongation of domain shape (e.g., Figure 3-5A).

\subsubsection{Evaluation of Joint Probabilities of Co-Occurrence of Model Terminals and Model Spines}

We considered that intra-areal integration could also be facilitated by the increased probability of terminals of a common preference coinciding with the spines of neurons associated with another functional preference. Integration in this latter case would involve the strong modulation of a neuron (with one functional class preference) by activated terminals of neurons of another functional class. To investigate whether domain proximity could also support elevated co-occurrence of differently preferring terminal and spines in our model, we introduced three different constants describing spatial decay of spine density, $\mathrm{k}_{\mathrm{S}}=25,50$ and $75 \mu \mathrm{m}$. Terminal distribution was not changed $\left(\mathrm{k}_{\mathrm{t}}=100 \mu \mathrm{m}\right)$. We assigned 1200 spines per model cell, the 
same total assigned for terminals per cell. Note that the spine distribution definition and calculation of joint probability of spines with terminals were the same as those used in terminal distribution, the difference only being the spatial decay constant $\left(k_{s}\right)$, which typically was defined less than $\mathrm{k}_{\mathrm{t}}$. A color preferring domain and an orientation preferring domain at three different center-center separations $\left(d_{P}=600,800\right.$ and $\left.1000 \mu m\right)$ were specified. Following 100 randomizations, we calculated the joint probability of terminals (preferring the same color as the color-preferring domain) and spines (preferring the same orientation as the orientation preferring domain).

A.

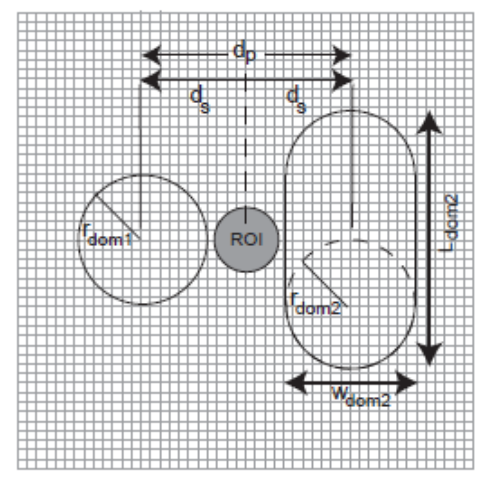

B.

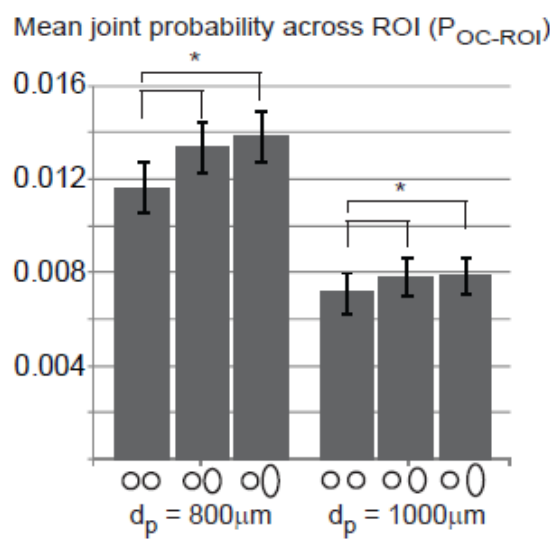

C.

Proportion of ROI cells with $\mathrm{P}_{\mathrm{OC}}>\mathrm{P}_{\text {Oc99s }}$

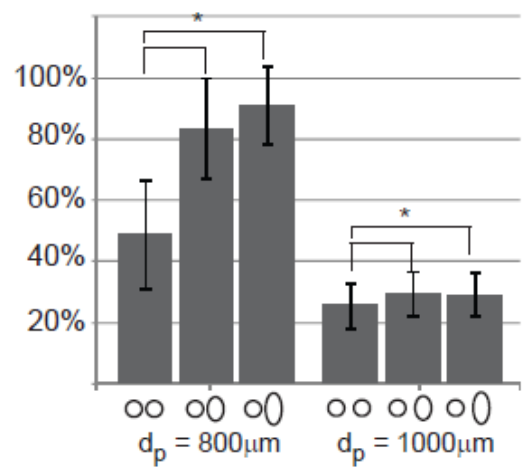

Figure 3-5. Cluster elongation further elevates POC. (A) To investigate the role that domain shape may play in modulating POC, paired domain configurations included either a shorter elongated form (width, Wdom $2=500 \mu \mathrm{m}$; length, Ldom2 $=750 \mu \mathrm{m})$ or longer elongated form $(\mathrm{Wdom} 2=500 \mu \mathrm{m}$; Ldom2 $=1000 \mu \mathrm{m})$, paired with a circular domain $($ rdom $1=250 \mu \mathrm{m})$. Compared with non-elongated pairs, domain elongation significantly increased POCROI over 100 randomizations for separation distances $(\mathrm{dp})>=800 \mu \mathrm{m}$, (B), with more than $20 \%$ increase at $800 \mu \mathrm{m}$ separation. The proportions of ROI cells with POC>POC99S also significantly increased, (C). There were no significant increases in POC-ROI when configurations with shorter and longer elongated forms were compared. * $=$ ANOVA $p<0.0001$; Dunnett's posthoc $\mathrm{p}<0.01$. Error bars $=+/-1 \mathrm{SD}$. Color-coded map examples shown in Figure 3-6D.

\subsubsection{Control Study: Variation of Domain Purity, Terminal Density and Bias Parameters}

To investigate whether observed enhancements in $\mathrm{P}_{\mathrm{OC}-\mathrm{ROI}}$ were sensitive to parameter variation, we also repeated our quantifications for a subset of paired domain configurations $\left(\mathrm{d}_{\mathrm{p}}=\right.$ 600 and $800 \mu \mathrm{m}$ ) with variations in key parameters. In separate control experiments, the purity of our uniform domains was reduced from $100 \%$ to $80 \%$ and $60 \%$ by adding graded amounts of random preference. The terminal density was increased by an order of magnitude from 1,200 to 12,000 terminals/cell. Finally, the input bias (\% of terminals that were not associated with intraareal projection) was reduced from 0.1 to 0.05 , and then increased to 0.2 and 0.3 . $\mathrm{P}_{\mathrm{OC}-\mathrm{ROI}}$ 
distributions associated with these variations were compared with those obtained with our standard parameter settings ( $\mathrm{n}=100$ iterations). Only one parameter was varied in each control experiment.

\subsubsection{Control Study: Diffuse Vs. Patchy Projection Distributions}

The modeling experiments presumed all intra-areal terminal distributions were radially diffuse processes with exponentially declining distribution densities. These distributions were chosen as sufficient first approximations of the local connectivity patterns present in cortex. However we also recognize that a finite component of neuronal projection involves a 'patchy' distribution that can sometimes extend (with high density) several millimeters in V2 (e.g., see Figures 6 and 8, in Amir et al 1993). As an additional control experiment we also systematically reduced the proportion of terminals projected according to a radially diffuse distribution, while increasing the proportion of overall terminals distributed in a patchy distribution. How patchy projections are mapped in V2 (and their precise contribution as proportion of overall distributed terminals) is still not known in detail. We used Amir et al (1993) data (Figs 6, 8 and 17) based on biocytin tracer experiments in macaque monkey as the most comprehensive first approximation for patchy distribution parameters in our V2 domain model. These data are based on small biocytin tracer injections in macaque cortex, (Amir et al., 1993) As a first approximation of patchy redistribution, we divided our model cortical array into modules of $400 \mathrm{x} 400 \mu \mathrm{m}$, and defined the identical (randomly obtained) patchy distribution pattern for all cells within each module (12 circular terminal patches distributed per cell, $200 \mu \mathrm{m}$ patch diameter). To emulate Amir et al data we defined a patch randomization rule: $50 \%$ of patches located within a range $400-1000 \mu \mathrm{m}$, remaining $50 \%$ of patches located within a range of $1000-2100 \mu \mathrm{m}$ of module centers. Terminals assigned to patchy distributions were evenly distributed across all patches. We varied the proportion of total terminals associated with patchy projections ( $25 \%, 50 \%$ and 75\%; with the balance allocated to a radially diffuse distribution) and compared the obtained POC-ROI with configurations where there were no patchy projections defined. Array size was increased to 220 x 220 cells to accommodate greater distribution reach, however, data shown and analyzed was always cropped to the central 100x100 cells in the array. 


\subsection{Model 1 Results}

\subsubsection{Configuration A: Random (No Domain) Organization}

We began by first asking to what extent neurons are likely to exhibit significant integration of different response properties when they are not organized into spatially distributed functional domains, that is to say when preferences were always randomly distributed across the array (Figure 3-2A). One hundred different versions of these random configurations were generated. From these results, the $\mathrm{P}_{\mathrm{OC}}$ at each array coordinate and $\mathrm{P}_{\mathrm{OC}-\mathrm{ROI}}$ for all randomizations were determined. These $\mathrm{P}_{\mathrm{OC}}$ across the array were plotted and inspected as 2D thresholded intensity-coded (Figure 3-2B) and color-coded maps (Figure 3-6A). Although no spatial

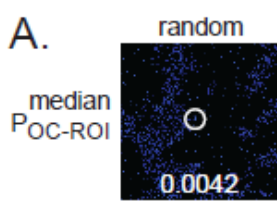

$$
\text { B. }
$$
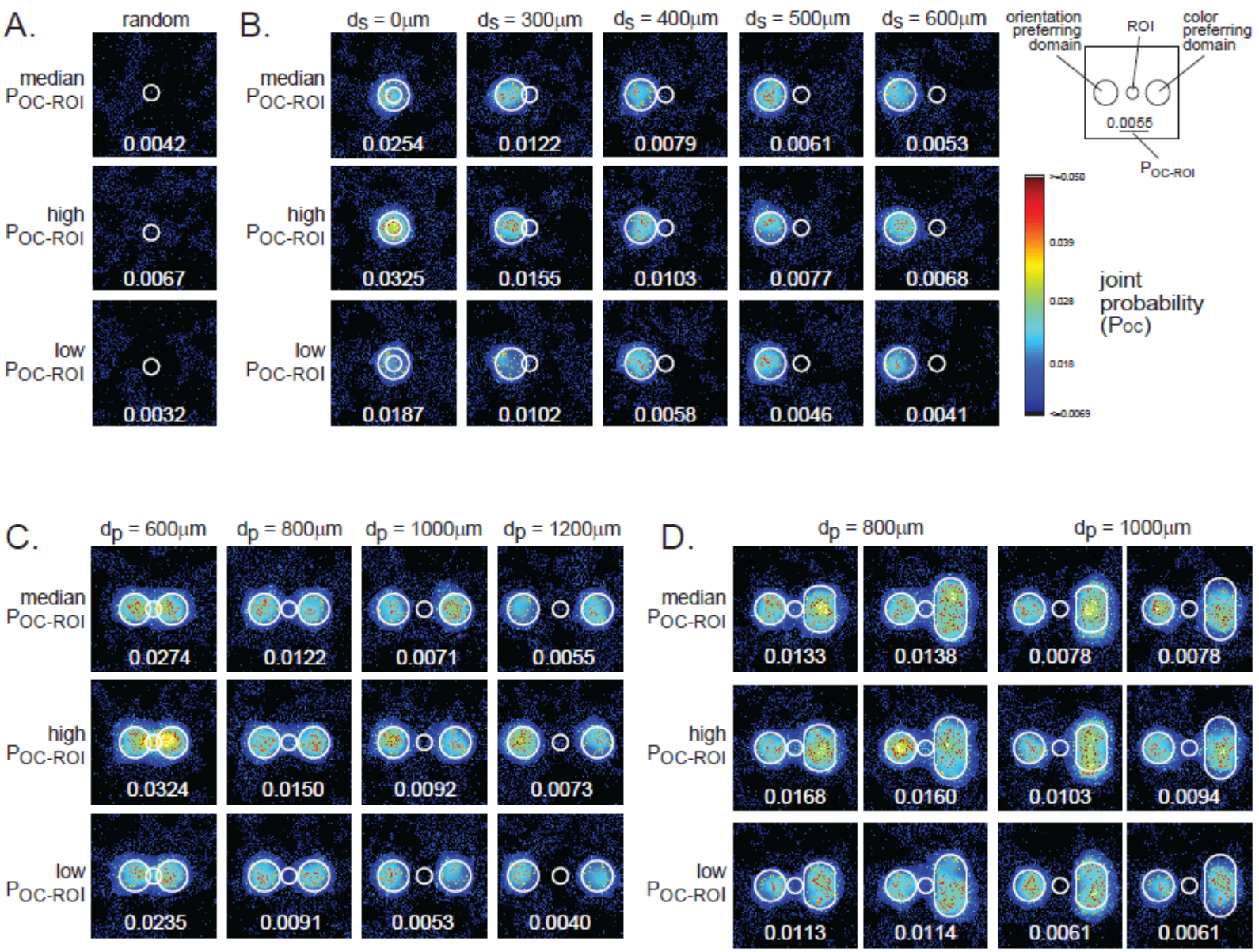

Figure 3-6. Color-coded POC examples for random, single domain and paired domain configurations. Colorcoding available in on-line versions only, refer legend, top right. Obtained POC-ROI in centrally placed ROI is superimposed over each map (white text). Selection criteria: median POC-ROI, high POC-ROI (within top 3 percent of obtained POC-ROI values for 100 randomizations), low POC-ROI (within bottom 3 percent). (A) Random (no domain) configurations. (B) Single circular domain configurations, orientation domain at central $(\mathrm{ds}=0)$ or left-placed locations ( $\mathrm{ds}=300-600 \mu \mathrm{m})$. (C) Paired circular domain configurations, orientation domain at left-placed location, color domain at right-placed location at varying center-center separations $(\mathrm{dp}=600-1200 \mu \mathrm{m})$. (D) Paired circular and elongated domain configurations (shorter elongated and longer elongated, $\mathrm{dp}=800$ and $1000 \mu \mathrm{m})$. 
concentration of color or orientation preferences were specifically defined by formal domain specification, random configurations nevertheless exhibited surprising cloud-like concentrations (approximately $250-1000 \mu \mathrm{m}$ in breadth), consisting of higher (or lower) than average joint probabilities. Since these variations could lead to a range of average joint probabilities within defined ROI, we sought to determine the overall likely distribution of $\mathrm{P}_{\mathrm{OC}-\mathrm{ROI}}$ values. Twentyfive hundred independent randomizations (Figure 3-2C), revealed a bell-shaped distribution of $\mathrm{P}_{\mathrm{OC}-\mathrm{ROI}}$ that was not significantly different from a normal distribution. However there was a slight variation from normal below the distribution mode (skewness $=0.325$ ), perhaps indicative of a thresholding effect associated with spatial mass action. We calculated the $99^{\text {th }}$ percentile value for joint probability $\left(\mathrm{P}_{\mathrm{OC} 99}\right)$ in this distribution of $\mathrm{P}_{\mathrm{OC}-\mathrm{ROI}}$, and other standard distribution metrics for all $\mathrm{P}_{\mathrm{OC}-\mathrm{ROI}}\left(\mathrm{P}_{\mathrm{OC} 99}=0.0069\right.$, mean $\mathrm{P}_{\mathrm{OC}-\mathrm{ROI}}=0.0049$, median $\mathrm{P}_{\mathrm{OC}-\mathrm{ROI}}=0.0048$, standard deviation $\mathrm{P}_{\mathrm{OC}-\mathrm{ROI}}=0.0007$, distribution $95 \%$ upper confidence limit $($ mean $+1.96 \mathrm{sd})=0.0062$, distribution 99\% upper confidence limit $($ mean $+2.58 \mathrm{sd})=0.0066)$. The 99\% upper confidence limits varied slightly by the $4^{\text {th }}$ decimal place when calculated by these two methods, so we used the higher (more conservative) $\mathrm{P}_{\mathrm{OCg9}}$ value (0.0069) as our baseline reference value to indicate higher than expected $\mathrm{P}_{\mathrm{OC}-\mathrm{ROI}}$ in later modeling configurations.

\subsubsection{Configuration B: Single Domain Organization}

We next investigated the role that the presence of a single functional domain may play in increasing $\mathrm{P}_{\mathrm{OC}}$ and $\mathrm{P}_{\mathrm{OC}-\mathrm{ROI}}$ when compared with 'random only' configurations. In particular, we asked if clustered preference model configurations would lead to significantly greater $\mathrm{P}_{\mathrm{OC}-\mathrm{ROI}}$ compared to the random configurations. A single, circular domain (250 $\mu \mathrm{m}$ radius) was predefined (Figure 3-3A) and cells within this contiguous sub-region were seeded as only one of the eight possible orientation preferences (with color preference randomly assigned). Cells outside the defined domain area were randomly assigned color and orientation preferences. To investigate how the location of this orientation preference domain influences $\mathrm{P}_{\mathrm{OC}}$ in our ROI at the center of the array, the domain center was placed at varying distances $\left(d_{\mathrm{S}}\right)$, ranging from $300 \mu \mathrm{m}$ to $600 \mu \mathrm{m}$ from the ROI center. In addition to these distances, $\mathrm{P}_{\mathrm{OC}}$ and $\mathrm{P}_{\mathrm{OC}-\mathrm{ROI}}$ were also

calculated when domain and ROI centers were superimposed $\left(d_{S}=0 \mu m\right)$. One hundred randomizations were generated for each placement distance and we determined $\mathrm{P}_{\mathrm{OC}}$ and $\mathrm{P}_{\mathrm{OC}-\mathrm{ROI}}$ in each case. Obtained $\mathrm{P}_{\mathrm{OC}}$ were plotted in 2-D thresholded intensity-coded (Figure 3-3B) and 
color-coded maps (Figure 3-6B). P $_{\mathrm{OC}-\mathrm{ROI}}$ were distributed normally (see Figure 3-3C, for $\mathrm{d}_{\mathrm{S}}=$ $0 \mu \mathrm{m}$ and $\mathrm{d}_{\mathrm{S}}=600 \mu \mathrm{m}$ for 2500 iterations (black) superimposed over normal distributions (grey)). A one-way ANOVA was used to test for $\mathrm{P}_{\mathrm{OC}-\mathrm{ROI}}$ differences between these single domain configurations and random-only configurations. $\mathrm{P}_{\mathrm{OC}-\mathrm{ROI}}$ differed significantly across all distances $(F(5,599)=3926, p<0.0001)$ (Figure 3-3D). Post-hoc analysis using Dunnett's test with random configuration condition as a contrast control revealed that $\mathrm{P}_{\mathrm{OC}-\mathrm{ROI}}$ at all five distances of $\mathrm{d}_{\mathrm{S}}=0$, $300,400,500$, and 600 were significantly greater than for a random configuration $(p<0.01)$. We used an additional alternative approach to test for significant effects of the introduction of a single domain by calculating the proportion of cells within the ROI that had $\mathrm{P}_{\mathrm{OC}}$ values exceeding $\mathrm{P}_{\mathrm{OCg9}}$. A one-way ANOVA was used to test for differences in these proportions between the single domain configurations and random-only configuration. Obtained proportions differed significantly across all distances $(F(5,599)=1672, p<0.0001)$ (Figure 3-3E). Post-hoc analysis using Dunnett's test revealed that calculated proportions at all five distances of $d_{s}=0$, $300,400,500$, and 600 were significantly greater than for a random configuration $(p<0.01)$. These latter data suggested that increased probabilities are not simply due to large increases in a relatively few cells. Notably, we found that $\mathrm{P}_{\mathrm{OC}-\mathrm{ROI}}$ was highest when the ROI and the defined single domain shared common centers (i.e. $d_{S}=0$ ). This suggests that domain organization in itself could contribute significantly to increased local integration of orientation and color within its confines. More importantly, however, these findings support the hypothesis that when neurons organize according to a single preference (e.g., either color or orientation), then nearby regions also have a significantly greater chance of receiving terminals that have preferences matching the domain. For example, if a domain exhibits preference for horizontal orientation, then these data suggest that immediately neighboring regions would show an increased cooccurrence of terminals with a horizontal orientation preference and terminals with any color preference.

\subsubsection{Configuration C: Paired Domain Organization}

The results obtained from Configuration B analyses indicated the inclusion of a single domain organization in our model system could lead to a facilitation of localized functional integration, when compared with a random preference organization. However, functional imaging and electrophysiological experiments in primate V2 and other visual cortical areas have 
shown that functional domains do not usually exist in isolation. Rather, they usually exist in proximity to other domains with different functional preference, such as a neuronal domain organized with regards to color preference in the vicinity of an orientation preferring domain (as observed across the thin/pale stripes of macaque V2). Since single domain organization already offers a mechanism for increased efficiency in integration of function, we reasoned that paired domain organization could provide a further mechanism for enhancing probability of integration. In particular we hypothesized that integration of the primary stimulus preferences associated with each domain would be selectively facilitated. Using the same general 100x100 cells model design as Configurations $\mathrm{A}$ and $\mathrm{B}$, we seeded two circular domains (with either domain possessing a color or an orientation organization preference) (Figure 3-4A) and again determined $\mathrm{P}_{\mathrm{OC}}$ and $\mathrm{P}_{\mathrm{OC}-\mathrm{ROI}}$. The distances between domain centers were systematically varied $\left(\mathrm{d}_{\mathrm{P}}=600\right.$ $1200 \mu \mathrm{m})$ while keeping both domains equidistant from the array center and ROI center. Examples of obtained $\mathrm{P}_{\mathrm{OC}}$ values were again plotted in 2-D thresholded intensity (Figure 3-4B) and color-coded maps (Figure 3-6C). Joint probability values were then obtained for these paired domain configurations and compared with single domain and random configurations. It is important to note that $d_{P}$ is defined as the distance between the two domain centers and thus twice the distance between a single domain center and the ROI center as defined in single domain configurations (i.e., $\mathrm{d}_{\mathrm{P}}=2 \mathrm{x} \mathrm{d}_{\mathrm{S}}$ ). Thus joint probabilities obtained at $\mathrm{d}_{\mathrm{P}}=600 \mu \mathrm{m}$ in paired domain configurations were appropriately compared with joint probabilities at $\mathrm{d}_{\mathrm{S}}=$ $300 \mu \mathrm{m}$ in single domain configurations (refer to Figures 3-3A and 3-4A). $\mathrm{P}_{\mathrm{OC}-\mathrm{ROI}}$ values were normally distributed (see example distributions at $d_{p}=400,600$ and $800 \mu m$, Figure 3-4C). POCROI was significantly higher in paired configurations compared to both random and single domain configurations. A one-way ANOVA was used to test for differences in $\mathrm{P}_{\text {OC-ROI }}$ across all paired domain configurations tested and the random configurations. $\mathrm{P}_{\mathrm{OC}-\mathrm{ROI}}$ for paired and random configurations differed significantly across all distances investigated $(F(7,799)=6009, p$ $<0.0001$ ) (Figure 3-4D). Post-hoc analysis using Dunnett's test revealed that $\mathrm{P}_{\mathrm{OC}-\mathrm{ROI}}$ for all seven separations of paired domains $\left(d_{P}=600-1200 \mu m\right)$ were significantly greater than for a random

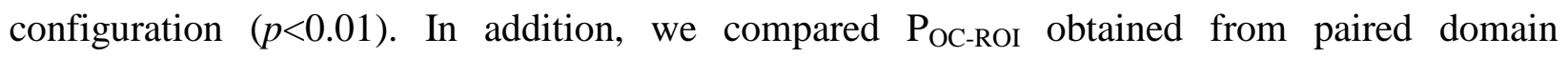
configurations at each tested separation against $\mathrm{P}_{\mathrm{OC}-\mathrm{ROI}}$ obtained from single domain configurations at equivalent distances. Paired domain configurations yielded significantly greater $\mathrm{P}_{\mathrm{OC}-\mathrm{ROI}}$ than single domains for all separations (t-Test: Two-Sample Assuming Equal Variances: 
$\left.\mathrm{d}_{\mathrm{P}}=600-1100 \mu \mathrm{m}, \mathrm{p}<0.0001 ; \mathrm{d}_{\mathrm{P}}=1200 \mu \mathrm{m}, \mathrm{p}<0.05\right)$. We also examined whether paired domain configurations exhibited higher than expected proportions of ROI cells with high $\mathrm{P}_{\mathrm{OC}}$. The $99^{\text {th }}$ percentile values of $\mathrm{P}_{\mathrm{OC}-\mathrm{ROI}}$ for single domain configurations for each $\mathrm{d}_{\mathrm{S}}$ ( $\mathrm{P}_{\mathrm{OCg}}$ ) were determined and the proportions of cells that exceeded these thresholds were quantified. The obtained proportions in our paired configurations were compared with those obtained from comparable single domain configurations (Figure 3-4E). There were significant increases in the proportion of ROI locations meeting criterion of $\mathrm{P}_{\mathrm{OC}}>\mathrm{P}_{\mathrm{OC} 995}$ when compared with comparable single domain configurations (t-Test: Two-Sample Assuming Equal Variances: $d_{P}=600-1100 \mu m$ $\left.\mathrm{p}<0.001 ; \mathrm{d}_{\mathrm{P}}=1200 \mu \mathrm{m}, \mathrm{p}<0.01\right)$. In other words, when model orientation-preferring and colorpreferring domain centers were separated by distances of up to $1200 \mu \mathrm{m}$ (as can be found in monkey Area V2), there was a significant increase in $\mathrm{P}_{\mathrm{OC}}$. The joint probability is elevated for the orientation/color combination of the nearby uniform domains.

If paired domain organization could give rise to elevated joint probabilities at extensive distances between functional domains, we wondered to what extent probability enhancements might also occur at the functional domain's center. Again we configured paired domains in our model at different separations, but rather than placing the ROI between the domain pairs, the ROI was placed at the center of the left-placed domain. We performed 100 consecutive randomizations, and then compared obtained probabilities against those from further configurations where there was no neighboring domain. With the presence of a second domain there were significant increases in $\mathrm{P}_{\mathrm{OC}-\mathrm{ROI}}$ (t-Test: Two-sample assuming equal variances, $\mathrm{p}<0.001$ ). This enhancement was greater than $10 \%$ on average for $d_{p}=500 \mu m$. In summary, Configuration $\mathrm{C}$ results indicate that paired model domains are associated with elevated joint probabilities in at least two spatial locations relative to the domains: mid-way between differently preferring uniform domains, and at the centers of the domains.

\subsubsection{Configuration D: Elongated Domain Shape}

Functional imaging and electrophysiological data recorded from non-human primates V2 has suggested that the geometry for functional domains, such as color- and orientation-preferring domains, need not be confined to circular shapes. Rather, they could also be elongated forms (e.g., refer Figure 6 in Ramsden et al. 2001; Tso et al. 2001). To investigate the geometric implications of domain lengthening on potential inter-domain functional integration, elongated 
domain shapes were introduced in a final reconfiguration of Model 1(Figure 3-5A). Again, 100 randomized iterations were obtained using configurations comprising two of the three possible domain shapes: a circular domain with a $250 \mu \mathrm{m}$ radius, an elongated domain with a width of $500 \mu \mathrm{m}$, and a length of $750 \mu \mathrm{m}$, and a second, longer elongated domain of $500 \mu \mathrm{m}$ by $1000 \mu \mathrm{m}$. $\mathrm{P}_{\mathrm{OC}}$ values were plotted in 2-D arrays for two domain separations $\left(\mathrm{d}_{\mathrm{P}}=800 \mu \mathrm{m}\right.$ and $\left.1000 \mu \mathrm{m}\right)$ (Figure 3-6D). Separations of 800 and $1000 \mu \mathrm{m}$ were chosen because these exhibited moderate elevations in joint probability for paired circular domain configurations and thus allowed range for further putative enhancement due to domain shape elongation. A one-way ANOVA revealed $\mathrm{P}_{\text {OC-ROI }}$ differences among these three different pairings at $800 \mu \mathrm{m}$ and $1000 \mu \mathrm{m}$ inter-domain distances $(F(2,299)=114.35, p<0.0001$ and $F(2,299)=24.93, \mathrm{p}<0.0001)$. Dunnett's post-hoc analysis revealed that $\mathrm{P}_{\mathrm{OC}-\mathrm{ROI}}$ was significantly greater when either a short elongated or longer elongated form was present compared with circle only pairs (Figure $3-5 B)(p<0.01)$. There were no significant differences between configurations with shorter and longer elongated domains, however. Inclusion of elongated domain forms significantly elevated the proportion of ROI cells where $\mathrm{P}_{\mathrm{OC}}>\mathrm{P}_{\mathrm{OC} 99 \mathrm{~s}}$ (One way ANOVA, $\mathrm{d}_{\mathrm{P}}=800 \mu \mathrm{m}$ : $F(2,299)=203.69, p<0.0001$; Dunnett's post-hoc test, $p<0.01$; dp=1000 $\mu \mathrm{m}$ : and $F(2,299)=24.93, p<0.001$; Dunnett's post-hoc test, $p$ $<0.01$ ) (Figure 3-5C). Note that the change in shape from circular to elongated form almost doubled the proportion of cells above P $_{\text {OC99 }}$ threshold on average with separations of $800 \mu \mathrm{m}$. In summary, we found that domain elongation could foster further increases in probability of cooccurrence of terminals with primary preference characteristics of the domain pairs.

\subsubsection{Joint Probability of Model Terminal and Spines in Paired Domain Configurations}

Our results indicated domain dependent increases in joint probabilities of the different terminal classes associated with defined model domains, but we reasoned that it would be also relevant to investigate the joint probabilities of model terminals and spines. We tested this in paired domain configurations for 3 different spine distribution spatial time constants $\mathrm{k}_{\mathrm{S}}=25,50$ and $75 \mu \mathrm{m}$. A one-way ANOVA was used to test for joint probability differences among the three domain separations. Compared with random configurations, paired domains yielded significantly elevated terminal and spine joint probabilities at every separation tested and for every spine constant tested (Figures 3-7A and 3-7B). Joint probabilities for terminal and spine differed 
A.

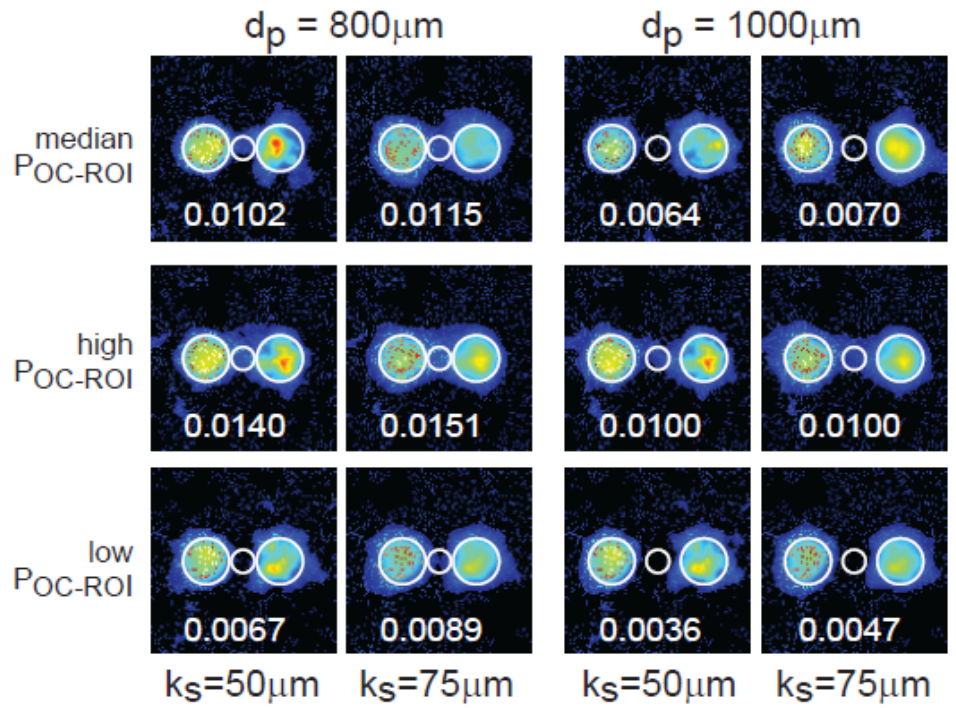

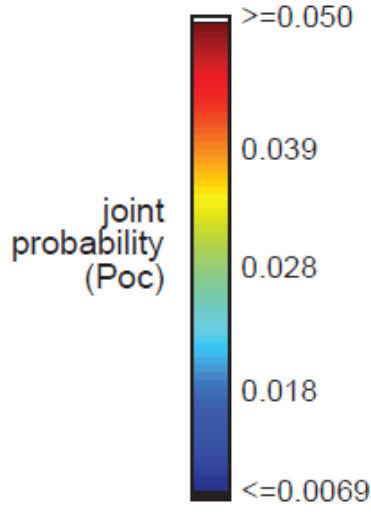

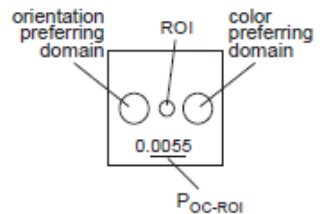

B.

Mean joint probability across ROI (POC-ROI)

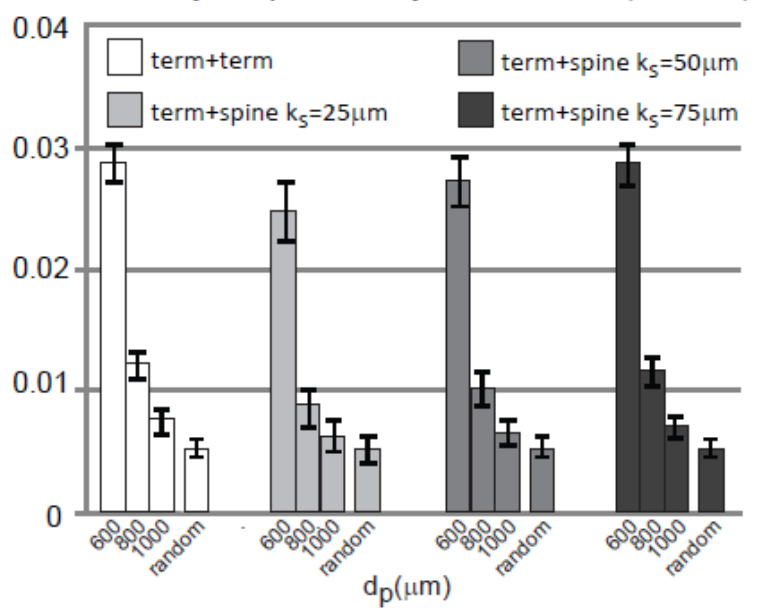

C. Mean joint probability across ROI (POc-ROI)

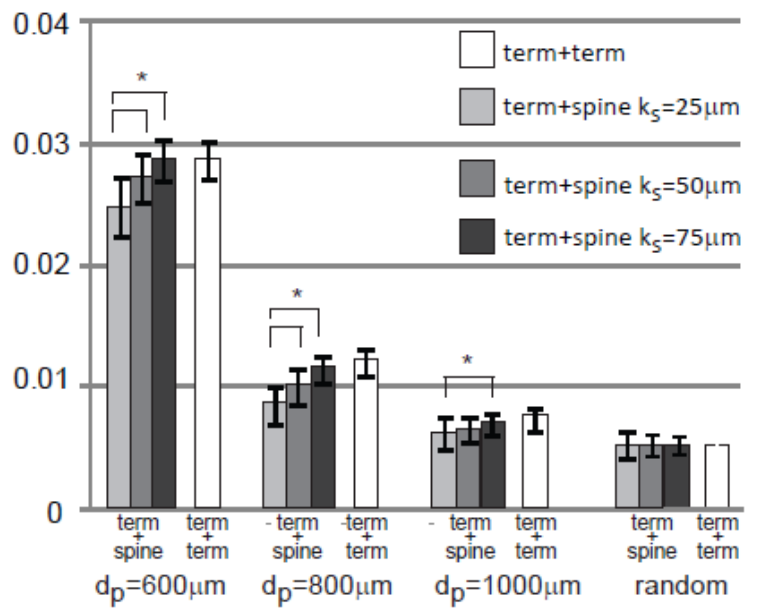

Figure 3-7. Domain proximity increased joint probability of color-preferring terminals and orientation preferring spines. To investigate whether domain proximity could also support elevated co-occurrence of differently preferring terminal and spines in our model, we introduced three different spine spatial decay constants, $\mathrm{ks}=25,50$ and $75 \mu \mathrm{m}$. Terminal distribution remained constant $(\mathrm{kt}=100 \mu \mathrm{m})$. (A) Color-coded examples of joint probability maps of terminals and spines in paired domain configurations. Orientation domain at left-placed location, color domain at right-placed location at varying center-center separations $(\mathrm{dp}=800,1000 \mu \mathrm{m})$. Color-coding available in on-line versions only, refer legend, bottom left. The average POC value for cells located within a central ROI (POC-ROI) is superimposed over each POC map. Examples were selected from 100 consecutive randomizations for each configuration, median POC-ROI, high POC-ROI (within top 3 percent of obtained POCROI values for each configuration), low POC-ROI (within bottom 3 percent of obtained POC-ROI values for each configuration. (B) Compared with random configurations, paired circular domains (radius 250 $\mu \mathrm{m}$ ) yielded significantly elevated terminal and spine joint probabilities at every separation tested $(\mathrm{dp}=600,800$ and $1000 \mu \mathrm{m})$ and for every spine constant tested (ANOVA $\mathrm{p}<0.0001$; Dunnett's posthoc $\mathrm{p}<0.01$ ). (C) A decrease in spine constant was associated with a decrease in joint probability, with $\mathrm{ks}=25 \mu \mathrm{m}$ yielding a significantly less POC-ROI than for highest ks in all pair cases (ANOVA $\mathrm{p}<0.0001$; Tukey's posthoc, ${ }^{*}=\mathrm{p}<0.01$ ). Note that Panel $\mathrm{C}$ is a rerepresentation of the data set shown in Panel B. Error bars $=+/-1 \mathrm{SD}$. 
significantly from random across the three separations for all spine constants (ANOVA $\mathrm{p}<0.0001$; Dunnett's post-hoc against random $\mathrm{p}<0.01$ ). A decrease in spine constant was associated with a corresponding decrease in joint probability, with $\mathrm{k}_{\mathrm{S}}=25 \mu \mathrm{m}$ yielding a significantly less $\mathrm{P}_{\mathrm{OC}-\mathrm{ROI}}$ than for larger $\mathrm{k}_{\mathrm{S}}$ in all cases (one way ANOVA $\mathrm{p}<0.0001$; Tukey's post-hoc, $\mathrm{p}<0.01$ ) (Figure 3-7C). Thus we report that despite substantial difference in the extent of model dendritic vs. axonal reach, paired domain configurations nevertheless exhibited significantly elevated joint probabilities of terminals coinciding with spines. This supports the hypothesis that significant inter-domain integration may be mediated by terminals arising from one domain onto spines of a neighboring domain (in addition to higher probabilities of convergent terminals from each domain).

\subsubsection{Functional Joint Probabilities Remain Enhanced Despite Primary Parameter Manipulations}

We further tested Model 1 to ensure that our results were not unduly biased by selective parameter specifications. First, we reduced the functional purity of domains used in paired configurations from $100 \%$ (i.e., all cells are seeded as preferred preference) to $80 \%$ or $60 \%$ (where a majority but not all domain cells were seeded with the preference of the domain and the remaining minority were instead allocated random preferences different from that of the domain). While there was some reduction in joint probability with decreasing purity for tested separations of 800 and $1000 \mu \mathrm{m}$, all obtained $\mathrm{P}_{\mathrm{OC}-\mathrm{ROI}}$ remained significantly greater than single domain controls (ANOVA $\mathrm{p}<0.0001$, post-hoc Dunnett's test with a single domain configuration condition as a contrast control $\mathrm{p}<0.01$ ) (Figure $3-8 \mathrm{~A}$ ). A second parameter control experiment increased terminal density by an order of magnitude (from 1200 to 12,000 terminals/cell). Again all obtained $\mathrm{P}_{\mathrm{OC}-\mathrm{ROI}}$ remained significantly greater than single domain controls despite an increase in density of an order of magnitude (ANOVA $\mathrm{p}<0.0001$, post-hoc Dunnett's test $\mathrm{p}<0.01)$. However, an increase in density from 1200 to 12,000 also led to a significant increase in P OC-ROI at $\mathrm{d}_{\mathrm{p}}=600 \mu \mathrm{m}$ (t-Test, $\mathrm{p}<0.001$ ) but not at $\mathrm{d}_{\mathrm{p}}=800 \mu \mathrm{m}$ (t-Test, $\mathrm{p}>0.05$ ) (Figure $3-8 \mathrm{~B}$ ). A third parameter control experiment varied the specification of input bias from 5\% through 30\% and obtained joint probabilities were compared with the $10 \%$ standard bias. All biases evoked significant increases in $\mathrm{P}_{\mathrm{OC} \_ \text {ROI }}$ joint probability, when compared to the single domain $10 \%$ 
A.

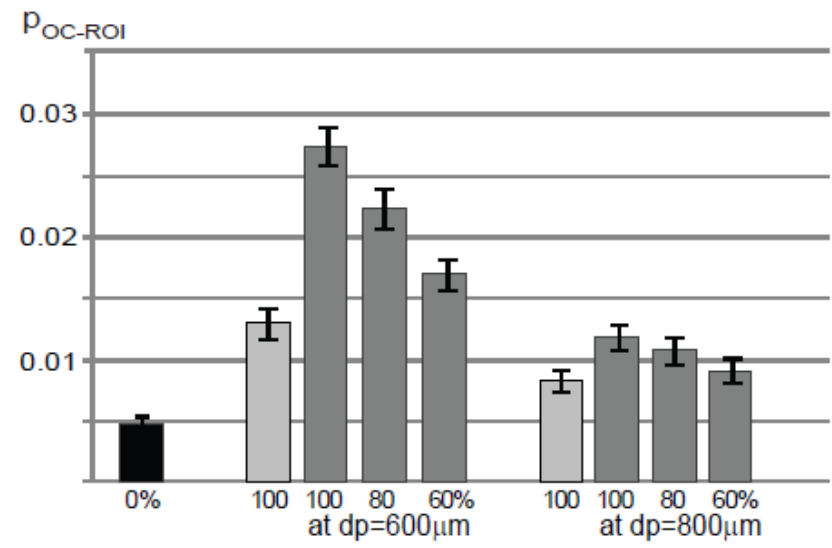

C.

vary domain purity

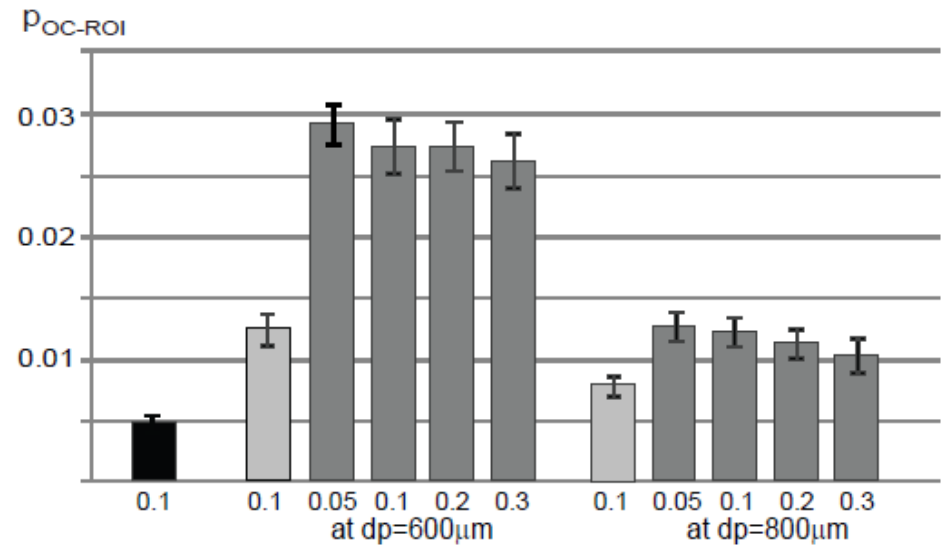

B.

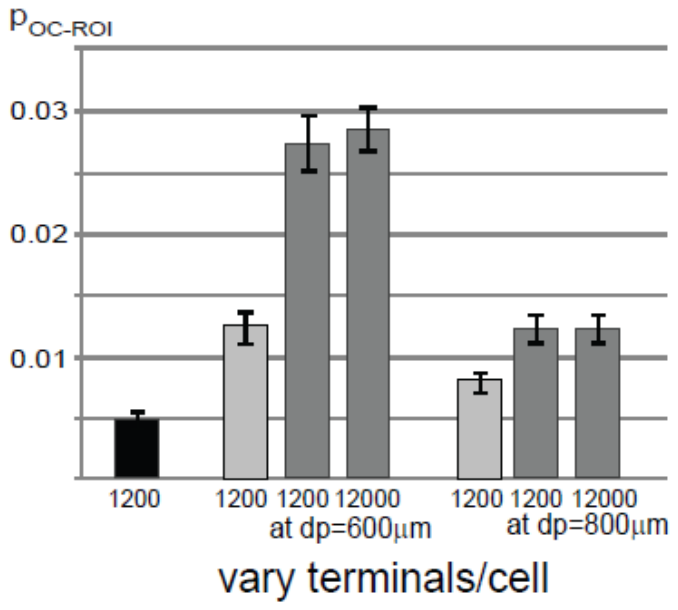

random control

single domain control

paired domain

Figure 3-8. Significant enhancement in joint probability is maintained despite substantial variations in domain purity, terminal density and bias. (A) The percentage of cells within domains matching the overall domain preference ('domain purity') was reduced from 100\% to 60\%. POC-ROI obtained from paired domain configurations ( $\mathrm{dp}=600$ and $800 \mu \mathrm{m})$ reduced as purity decreased but remained significantly greater than random (and single domain controls at equivalent distances) for all purities tested (ANOVA $p<0.0001$; Dunnett's posthoc $\mathrm{p}<0.01$ ). (B) Terminal density specification was increased by an order of magnitude from 1,200 to 12,000 per cell and POC-ROI remained significantly greater than random and single domain controls (ANOVA $\mathrm{p}<0.0001$; Dunnett's post hoc $\mathrm{p}<0.01)$. However, there was a significant increase in POC-ROI at $d p=600 \mu \mathrm{m}$ ( $\mathrm{t}$-Test, $\mathrm{p}<0.001)$ but not at $\mathrm{dp}=800 \mu \mathrm{m}$ (t-Test, $\mathrm{p}>0.05$ ) when terminal densities were varied. (C) Model bias varied from 0.05 to 0.3 resulted in a moderate reduction in POC-ROI but joint probabilities remained significantly greater than random and single domain controls (ANOVA $\mathrm{p}<0.0001$; Dunnett's posthoc $\mathrm{p}<0.01$ ). Significant differences were found between mean POC-ROI with respect to bias, with a significant decrease in POC-ROI as bias increased in all posthoc comparisons (ANOVA $\mathrm{p}<0.0001$, post hoc Tukey's, $\mathrm{p}<0.05$ for all bias cases, except when $\mathrm{dp}=600 \mu \mathrm{m}$, bias $0.1 \mathrm{vs}$. $0.2, \mathrm{p}>0.05)$. Error bars $=+/-1 \mathrm{SD}$.

control (ANOVA $\mathrm{p}<0.0001$, post-hoc Dunnett's test $\mathrm{p}<0.01$ ) (Figure 3-8C). Significant differences were found between mean $\mathrm{P}_{\mathrm{OC}-\mathrm{ROI}}$ with respect to bias, with a significant decrease in $\mathrm{P}_{\mathrm{OC}-\mathrm{ROI}}$ as bias increased in all post-hoc comparisons (ANOVA $\mathrm{p}<0.0001$, post-hoc Tukey's, $\mathrm{p}<0.05$ for all bias cases, except when $\mathrm{dp}=600 \mu \mathrm{m}$, bias 0.1 vs. $0.2, \mathrm{p}>0.05$ ). In conclusion, the 
results of these additional control experiments indicate that pronounced manipulations of our key model parameters do not change our conclusion regarding the propensity for paired domains to significantly elevate joint probabilities.

\subsubsection{Functional Joint Probabilities Remain Enhanced Despite an Increase in Terminal Patchy}

\section{Redistribution}

A.

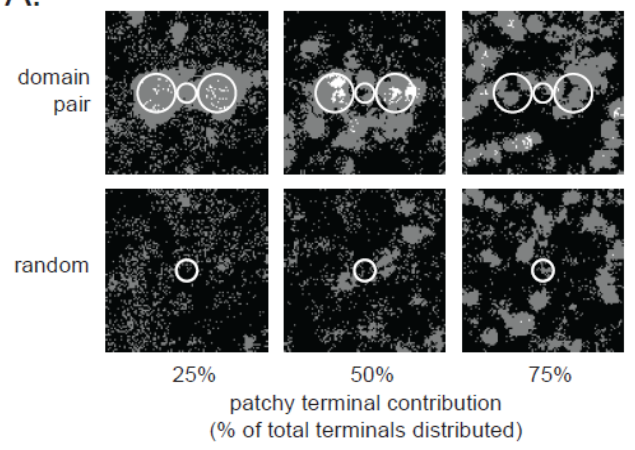

B.

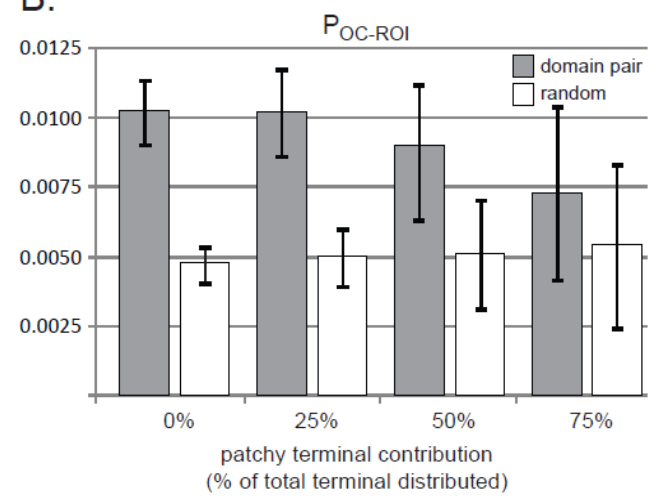

Figure 3-9. Elevated joint probabilities persist with patchy (cf. diffuse) terminal distribution. (A) Example POC maps (median POCROI) for domain pair configuration $(\mathrm{dp}=800 \mu \mathrm{m})$ with increasing patchy terminal projection contributions (25, 50 and $75 \%$ patchy projections relative to total terminal projections). As patchy terminal proportions increased, we observed increasing heterogeneities in the POC maps. Map greyscale codings same as Fig 3. (B) Variance in POC-ROI increased and mean POC-ROI decreased with progressive increases in patchy terminal proportions. However, at 25\%, 50\% and 75\% proportions, POC-ROI remained significantly greater for domain pair configurations than for random configurations (also with patchy projections) where no domains were defined (t-Test, $\mathrm{p}<0.0001)$. Error bars $=+/-1 S D$.

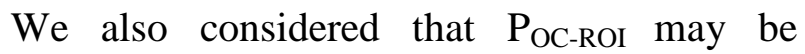
significantly influenced by our definition of radially diffuse (rather than patchy) terminal distributions. Therefore as an additional control study, proportions of total terminal counts were projected into patchy rather than diffuse distributions. Plausible patchy terminal distribution patterns were devised based on data obtained from tracer studies in macaque monkey V2 cortex experiments (Amir et al 1993). As the proportion of patchy projections increased, the $\mathrm{P}_{\mathrm{OC}}$ maps were observed to become more heterogeneous (Figure 3-9A) for both random and domain pair configurations. While mean $\mathrm{P}_{\mathrm{OC}-\mathrm{ROI}}$ enhancements were observed to be smaller (and $\mathrm{P}_{\text {OC-ROI }}$ variance larger) when patchy proportions exceeded 50\%, POCROI remained significantly greater for paired domain configurations than when compared with $\mathrm{P}_{\mathrm{OC}-\mathrm{ROI}}$ obtained with random configurations, even though $25 \%, 50 \%$ and $75 \%$ of terminals were redistributed according to a biologically based patchy distributions in these control experiments (Figure 3-9B). These data suggest that intra-areal integration as suggested by Model 1 data may be relatively resilient to incorporation of moderate proportions of patchy terminal distribution schema. 


\subsection{Model 2: A Topographical Model Framework Incorporating Multiple Domain Proximity}

If, as suggested by our Model 1 data, domain proximity can lead to significant increases in mixed color and orientation terminal spatial co-distribution (and thus a potential for greater integration), then we wondered whether this propensity could be practically utilized in visual processing. In particular for V2, we felt that intra-areal integration of domain output would only be functionally practical if these integrations met the criteria of completeness (i.e., integration is feasible across a sufficient proportion of all possible combinations of color and orientation). We reasoned that if only a minority of possible color/orientation domain pairs share proximity, then there would be an inappropriate advantage for some combinations. If so, then only a limited set of stimulus configurations could then be processed using this mechanism of intra-areal integration. On the other hand if the typical geometric layout of V2 stripes placed the full range of color and orientation domains within sufficient proximity, then there could be potential for 'complete' and comprehensive representation of facilitated integrations. A second model framework (Model 2) was therefore devised to investigate the potential for completeness of the range of color/orientation integrations associated with V2 stripe compartments containing either color- or orientation-preferring domains. We used the data obtained from Model 1 to objectively determine whether domains were sufficiently in proximity for significant increases in terminal co-occurrence. 


\subsection{Model 2 Methods}

The second model (Figure 3-10A) allowed the configuration of 64 color and orientation domains (8 domain rows $\mathrm{x} 8$ domain columns). The first and last three columns were randomly assigned domains with one of eight orientation preferences, representing two separate pale stripes. The inner, middle two columns collectively represented the thin stripe and were randomly assigned domains with one of eight color preferences. Alternate configurations using only four preference type per class were also investigated. Thus, by selectively configuring subregions of the network with either color- or orientation-preferring domains, the array of discrete domains could approximate the functional organizations found across two pale (orientation) and an intervening thin (color) stripe. We used two different domain packing scenarios: one had an idealized packing structure with no domain overlap (500 $\mu \mathrm{m}$ diameter domains at $500 \mu \mathrm{m}$ centercenter spacings, see Figure 3-10A, left), the other had the same idealized structure but incorporated a small amount of domain overlap $(500 \mu \mathrm{m}$ diameter domains at $400 \mu \mathrm{m}$ centercenter spacings, see Figure 3-10A, right).

To measure the likelihood of sufficient proximity for domains of different preference modalities (i.e. color- and orientation), we defined and calculated a 'proximity hit-rate', or the aggregate occurrence of the center point of two domains of different modalities existing within a defined distance, $r_{\text {prox }}$ (Figure 3-10B). Thus, a hit rate of $100 \%$ or $64 / 64$ indicated that all 8 colors domains were within a predefined distance of all 8 orientation domains. Proximity hit-rates were determined for two plausible inter-domain separations (center-to-center distances of $400 \mu \mathrm{m}$ and $500 \mu \mathrm{m}$ ) with $\mathrm{r}_{\text {prox }}$ ranging from $500 \mu \mathrm{m}$ to $1500 \mu \mathrm{m}$. To ensure that hit-rate calculations were not biased by a particular random domain seed pattern, 100 different consecutive preference seedings were randomly generated for each configuration. 
A.

$\mathrm{d}_{\mathrm{p}}=500 \mu \mathrm{m}$
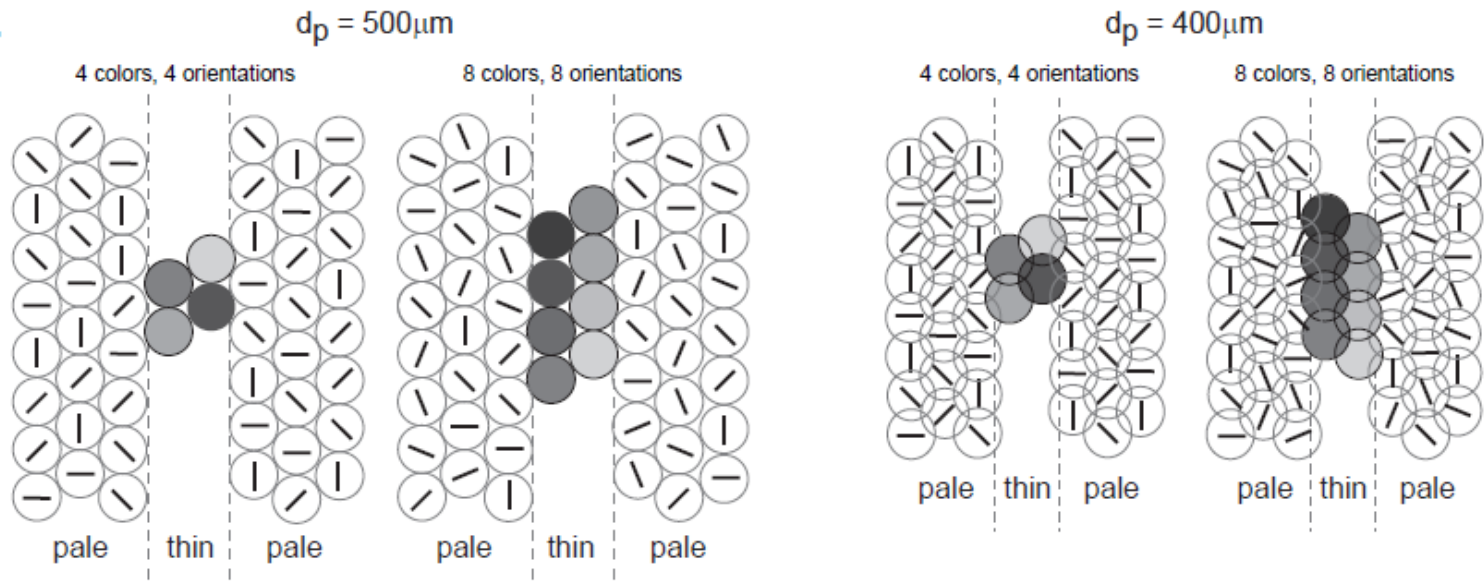

B.
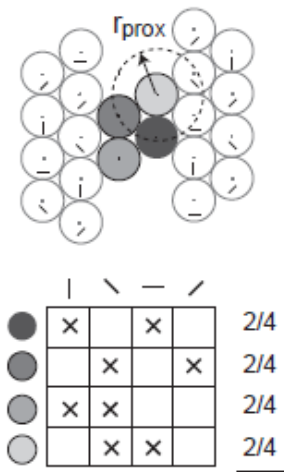

proximity hit-rate $=8 / 16=50 \%$

C.
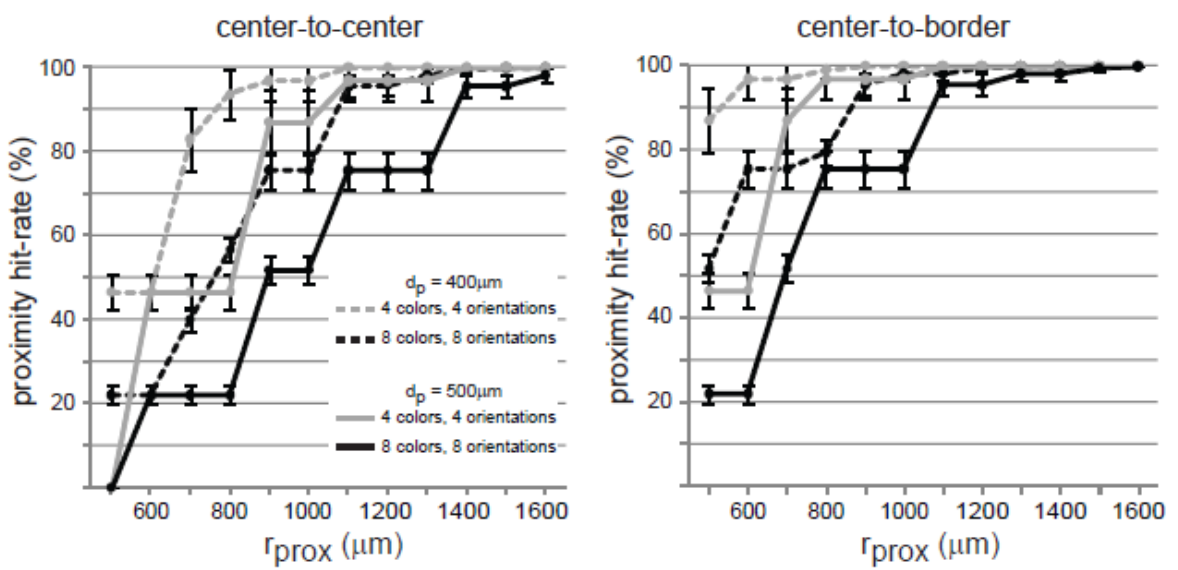

Figure 3-10. Large-scale packing constraints support comprehensive intra-areal integration. To investigate if orientation domains of all preferences might be sufficiently close enough to interact with color domains of all preferences via diffuse intra-areal mechanisms, we devised a second spatial model (Model 2) that incorporated representations of a single thin stripe and two flanking pale stripes in V2. Configurations were devised with 4 or 8 different randomly assigned preferences for color and orientation, and center-center packing distances of 400 or $500 \mu \mathrm{m}$, (A), with color and orientation domains constrained to thin and pale stripes respectively. Proximity hit-rates (percentage of all possible domain pair combinations within defined distances, rprox $=500-1600 \mu \mathrm{m}$ ) were calculated for each of 100 per configuration, (B). For 8 possible preferences, hit rate of $100 \%$ or 64/64 occurs if all 8 colors domains centers are within a predefined distance rprox of all 8 orientation domain centers. Mean center-center hit-rate increased as rprox increased, (C, left), for all configurations but was sensitive to spacing and number of preferences. With $500 \mu \mathrm{m}$ domain spacing, 8 color / 8 orientation configurations reached $90 \%$ criterion by rprox $=1400 \mu \mathrm{m}$, while 4 color $/ 4$ orientation configurations reached $90 \%$ criterion by $1100 \mu \mathrm{m}$. When spacing was reduced to $400 \mu \mathrm{m}$ however, 8 color / 8 orientation configurations reached $90 \%$ criterion by rprox $=1100 \mu \mathrm{m}$, and 4 color $/ 4$ orientation configurations reached $90 \%$ criterion by $800 \mu \mathrm{m}$. With reduced spacing, the distance ranges meeting $90 \%$ criteria using center-center measurements are consistent with those found to deliver significant joint probabilities in domain pair configurations of Model 1. When proximity rates were calculated less conservatively using distance from domain center to domain border (cf. domain center), hit-rates improved (C, right). With $500 \mu \mathrm{m}$ spacing, the 8 color $/ 8$ orientation configurations achieved $90 \%$ criterion by rprox $=1100 \mu \mathrm{m}$, but all other configurations at $400 \mu \mathrm{m}$ or $500 \mu \mathrm{m}$ spacings achieved $90 \%$ criterion by $900 \mu \mathrm{m}$ or less. Error bars $=+/-1 \mathrm{SD}$. 


\subsection{Model 2 Results}

As might be expected, hit rate increased as $r_{\text {prox }}$ increased (Figure 3-10C, left) for all configurations. However each configuration reached a mean hit rate $\geq 90 \%$ (i.e. $\geq 15 / 16$ for 4 classes; $\geq 58 / 64$ for 8 classes) within different $\mathrm{r}_{\text {prox }}$ specifications. With $500 \mu \mathrm{m}$ domain spacing, maps with eight color or orientation categories reached $90 \%$ criterion with $\mathrm{r}_{\text {prox }}$ of $1400 \mu \mathrm{m}$ (Figure 3-10C, left, black solid lines), while maps with four color or orientation categories reached $90 \%$ criterion by $1100 \mu \mathrm{m}$ (Figure 3-10C, left, grey solid lines). When domain spacing was reduced to $400 \mu \mathrm{m}$ however, maps with eight color or orientation categories reached $90 \%$ criterion by $1100 \mu \mathrm{m}$ (Figure 3-10C, left, black dotted lines) and maps with four color or orientation categories reached $90 \%$ criterion by $800 \mu \mathrm{m}$ (Figure 3-10C, left, grey dotted lines). The latter configuration also achieved $100 \%$ criteria within $1200 \mu \mathrm{m}$. Thus with relatively compact packing, our uniform color and orientation domains achieved relatively 'complete' hit rates down to submillimeter range. This range is consistent with those distances found to deliver significant joint probabilities in domain pair-configurations of Model 1. It is important to note that these hit-rate estimations conditionally depend on the specifications of packing and preference categorization. Additionally, the $d_{p}$ threshold for significant co-occurrence could be expected to further reduce if domain purity significantly declined or input bias significantly increased. Therefore the overall significance of our Model results depends on these parameter specifications. Our results are interpreted relative to Model 1 data based on center-to-center spacings, and therefore provide a relatively constrained estimate of overall proximities. As a comparison, we also recalculated hit rates based on a less conservative center-to-border metric (Figure 3-10C, right), and this indicated that peripheral portions (cf. centers) of all other domain categories were within surprisingly small reach. While the center-to-center significance statistics cannot be used to directly interpret the center-to-border hit rate data, these data also suggest potential for complete coverage via supplementary (but not necessarily radially diffuse) local terminal distribution patterns.

In summary, Model 2 indicated that the domain organization in V2 may feasibly place color- and orientation-preferring domains in sufficient proximity for a broad range of differently preferring terminal co-occurrences. The sufficient proximity (supporting a significant terminal co-occurrence than expected for a random organization) was determined from the results obtained in Model 1. 


\subsection{Discussion}

Using a computational modeling approach, we have systematically quantified the joint probability of differently preferring terminals arising from adjacent uniform domains. Our idealized models represent fundamental geometric aspects of functional organization in V2. These include the grouping of similarly responsive neurons into functional domains preferring a particular orientation or a particular color, and their relative spatial arrangements such that their member neurons have potential to project terminals to common intra-areal targets. Our results may be particularly relevant when different domain classes reside in proximity, as found with orientation-preferring domains in pale stripes flanking color preferring domains in thin stripes in V2. These data suggest four key relationships between domain organization and probability of functional convergence. First, the presence of a single domain preferring a given orientation (or color) could significantly increase the probability that terminals sharing that domain's preference will co-occur with other differently preferring color (or orientation) terminals. Our modeling results suggest that this significant probability increase could extend well beyond the spatial extent of the domain. Second, the co-occurrence of a particular orientation-preferring terminal and a particular color-orienting terminal may be further enhanced when neighboring functional domains also have these particular stimulus preferences. Third, domain shape elongation may further increase these probabilities. Fourth, the spatial scale and relative arrangements of V2 stripes and their functional domains could support a sufficiently complete set of possible functional combinations via intra-areal convergence. We initially hypothesized that domains and their proximity would increase joint probability of color/orientation-preferring terminal cooccurrence. We further hypothesized that the organization of the domains in a macaque V2-like cortex provides adequate circuitry for the different domains to interact and potentially results in a 'complete' representation of all co-occurrence combinations. Model 1 results suggest that our first hypothesis is supported: domain organizations increase joint probability of terminal cooccurrence. Based on data obtained from Model 1, the results from Model 2 suggest that our second hypothesis is conditionally supported: the overall organizational architecture could support a relatively complete coverage (>=90\%) given a sufficient (but biologically plausible) density of domain packing, terminal distribution and domain purity. In short, our model suggest that the domain organization, domain inter-distance and spacing of the stripes appear to afford a 
significant advantage for convergent inputs of different visual stimulus preference mediated within (rather than necessarily onto) V2.

\subsubsection{Mechanisms of Integration in V2: Inter-Areal vs. Intra-Areal}

That our results support the feasibility of intra-areal convergent integration is relevant because the general mechanism of convergent integration has typically been associated with inter-areal rather than intra-areal circuitry. Classical hierarchical models suggest that primary neuronal selectivity and receptive fields arise via converging projections from lower to higher areas (Hubel and Wiesel, 1962). Local circuitry, on the other hand, has usually been associated with contextual or inhibitory response modulations rather than convergent mechanisms per se (Gilbert, 1983; Callaway, 1998), or with consolidation of response through recurrent excitation (Douglas et al., 1995). It is important to note that there is currently not a consensus for how convergent inter-areal projections from V1 might contribute to combined color/orientation preferring responses in V2. A prevailing view has been that color and orientation attributes are largely processed separately by blob and interblob V1 compartments, respectively (Livingstone and Hubel, 1984), and that neural activity is passed from blobs to thin stripes, and from interblob to pale/thick stripes in V2 by distinct (not mixed) parallel pathways (Livingstone and Hubel, 1984; Hubel and Livingstone, 1987; Sincich and Horton, 2002). These parallel pathways might be expected to hinder rather than foster inter-areal integrative mechanisms in V2, leaving ample scope for V2's intra-areal circuitry to foster integration of color and orientation. However, there is other evidence that suggests blobs and interblobs may not project onto V2 in a distinct parallel schema. Guided by imaging and histological correlates, Roe and Ts'o (Roe and Ts'o, 1999) used cross correlation single unit electrophysiology to identify diverse patterns of functional connectivity between V1 and V2 neurons. They concluded that non-oriented color cells in V1 exhibit functional connectivity with color-oriented neurons in V2 when their receptive fields overlap. This suggests a mechanism whereby projections from non-oriented color cells in V1 might directly contribute to the construction of color-oriented responses in and near the thin stripe in V2. Recent neuroanatomical tracer studies also indicate that thin stripes potentially receive inputs from both blob and interblob V1 regions, and these circuits could contribute to the color-orientation responses in and near the thin stripe (Xiao and Felleman, 2004). These

experimental data offer some support for inter-areal mechanisms that might combine the 
separately represented color (blob) and orientation (inter-blob) information from V1 onto single color-oriented neurons in or at the border of thin stripes of V2. However other recent neuroanatomical tracer studies continue to support distinct and separate projections from V1 to V2 (blob to thin stripes; interblob to pale/thick stripes) (Sincich and Horton, 2002). A hierarchical inter-areal convergence mechanism underlying color/oriented response in V2 would seem less likely (and an intra-areal mechanism more likely) if color and orientation processing tend to be passed separately onto different V2 stripes from blob and inter-blobs in V1.

It is important to note that color-oriented neurons have been reported in V1 (Livingstone and Hubel, 1984; Roe and Ts'o, 1999; Johnson et al., 2001; Friedman et al., 2003). Using imaging, electrophysiological and anatomical methods, Landisman and Ts'o (Landisman and Ts'o, 2002b, a) also suggested that color oriented cells may be more prevalent at the gradually changing borders of the color selective blob and orientation selective interblob regions. These results suggest some combined color and orientation information from V1 may already be passed directly to V2 cells 'complete', presuming sufficient common targeting in V2. There are conflicting reports of the prevalence of color-oriented cells in V1 (ranging from relatively low (Livingstone and Hubel, 1984; Landisman and Ts'o, 2002b, a) to substantial (Friedman et al., 2003)). While some reports suggest that color-oriented V2 neurons are more prevalent at the pale/thin stripe border and may show specific clustered organization (Roe and Ts'o, 1995), other reports indicate that color-oriented tuning in all stripes is not rare (Gegenfurtner et al., 1996; Kiper et al., 1997; Shipp and Zeki, 2002) and may be generally quite prevalent in V2 (Friedman et al., 2003). If there are relatively few color-oriented neurons in V1, it seems unlikely that they could evoke color oriented selectivity in a substantial proportion of V2 cells in all stripes given the various projection schemas. In this case, we suggest that intra-areal mechanisms enhanced by relative domain organization could play a substantially constructive role. On the other hand, if color oriented V1 neurons are prevalent and these directly contribute to similarly mixed selectivity across V2, then we suggest that intra-areal mechanisms may be a means to further consolidate existing color oriented selectivity.

In contrast to possible feed forward mechanisms arising from V1, detailed extensive intrinsic circuitry exists in V2 and could also be a substrate for diverse intra-areal interactions between neurons and domains in the different stripe compartments (Levitt et al., 1994b; Levitt et al., 1994a; Tamura et al., 1996). The potential for potent intra-areal interaction and convergence 
is supported by recent reports (in the cat) that terminals arising from intra-areal connectivity are surprisingly predominant when compared to incoming inter-areal projections (Binzegger et al., 2004). Recent spatio-temporal modeling studies have suggested that border ownership properties of some V2 neurons may be derived from local V2 circuitry, although domain organization and proximity were not a focus of their model design (Zhaoping, 2005). Our data suggests two additional mechanisms may contribute to color/oriented responses in V2 and where intra-areal circuitry and clustered functional organization plays a key role. In these intra-areal mechanisms, neighboring functional domains that prefer either particular colors or orientations, and their associated local connections, could support a higher probability of color-orientation interactions than random distributions in V2. The first mechanism, intra-areal domain-mediated convergence, would involve a higher probability of spatially correlated color- and orientationpreferring terminals. The second mechanism, intra-areal domain-mediated modulation, would involve a higher probability of spatially correlated terminals and spines (color-preferring terminals on orientation preferring spines, or conversely orientation-preferring terminals on color-preferring spines). Our results show that paired model domains support localized enhancement of probability of co-occurrence of color and orientation preferring terminals. This probability enhancement remains significant to surprising large inter-domain spacings, up to $1 \mathrm{~mm}$ and more within our model. These effects are resilient to parameter manipulations. In V1, orientation domains are reported to be typically circular in form but by V2 the presence of elongated orientation domain forms can extend up to approximately $1 \mathrm{~mm}$ in length (Ramsden et al., 2001; Ts'o et al., 2001). Our results suggest that the change in domain shape from circular to elongated forms could further enhance the probability of inter-domain terminal convergence.

\subsubsection{An Explicit Role for Clustered Functional Organization?}

Our results suggest that effective intra-areal convergence could critically depend on domain organization as much as the presence of substantive intrinsic circuitry. The grouping of neurons with similar preference provides a concentrated local distribution of terminals with similar preference and thus can lead to effective increases in co-occurrence with other terminal concentrations. This concentration would be forfeited in a random organization (Configuration A). While concentration of terminals and spines does not guarantee synaptic formation, it is a conventional concept that increased densities at one location could give rise to higher probability 
of synapse formation (Stepanyants and Chklovskii, 2005). While we have not explored spatiotemporal mechanisms here it is likely that elevated intrinsic terminal co-distributions could selectively amplify integrated response via local recurrence mechanisms (Douglas et al., 1995). The role of cortical functional domains as explicit mediators of neural computation has been recently debated (Horton and Adams, 2005). It has been suggested that organized functional mappings may be a residue of developmental cortical competition (Kohonen, 1982; Piepenbrock and Obermayer, 2000), the consequence of a need for an optimal and uniform 'coverage' of functional representations (Swindale et al., 2000), or a prevailing need for the cortex to simply minimize wiring length (Koulakov and Chklovskii, 2001; Chen et al., 2006). Our model quantifications suggest that enhancement in probability of joint terminal co-occurrence (as a direct geometric consequence of neuronal groupings) may be one possible role that functional domains can play in neuronal computation.

It is important to clarify that our findings are not at all inconsistent with local interactions that would expect to be promoted by a wirelength minimization organizational schema (Swindale 1996, Koulakov and Chklovskii 2001). This is especially so, given that we have focused initially on radially diffuse projection distributions with exponentially declining falloff, a hallmark of some minimization schema. One way of looking at functional domain organization, however, is that clustering is simply an artefactual consequence of the wirelength minimization (rather than adding special functional merits per se). Our modeling experiments suggest that V2 domain organization (along with any associated wirelength minimization) can provide a sufficiently increased density of preference cells that can in turn provide a significantly increased density of selective terminal co-occurrence at common extra-domain sites. Importantly, while these findings also depend on an intra-areal wiring schema where neurons tend to connect locally, they fundamentally require that the neurons be sufficiently functionally clustered within a sufficient range. Our experiments primarily aimed to focus on and quantify the consequence of this functional clustering on terminal co-occurrences in relation to different domain shape, spacings and extra-domain locations known to occur in primate cortex. Thus, Model 1 comparisons were always made with and without functional domains, while at the same time always maintaining identical local wiring schema. 


\subsubsection{Permissive Advantages of Domain Organization and Larger Scale Stripe Geometry in V2}

If intra-areal mechanisms do play a practical role in visual processing of color and orientation integration, it would be expected that a complete range of color and orientation combinations should be permitted. When multiple color and orientation domains were arranged in a simplified pale/thin/pale model structure (Model 2), we found that domains were sufficiently close to each other to allow a relatively broad range of color-orientation combinations. These larger scale arrangements may therefore provide a means for efficient and sufficient common projections that allow the representation of all orientation/combinations without requiring an explicit alignment of two continuous maps (cf. (Swindale et al., 2000)). We suggest that domain organization per se permits specific integrations of different neural preferences that would be less probable in solely distributed organizations.

It is important to recognize that we found the complete representation of all possible color/orientation combinations critically depended on packing constraints and categorization, and therefore may be sensitive to other parameters such as domain purity and input bias. On the other hand our necessarily simplified model design may also underestimate the potential for complete integration. For example, when we also determined hit rate based on center-to-border metric (cf. center-to-center metric), a much larger percentage of domains became within reach at a smaller proximity range (Figure 3-10C, right). This suggests that further modeling studies with more complex domain and projection mappings may reveal significant and complete coverage according to criteria other than our (center-to-center based) Model 1 data.

\subsubsection{Choice of Parameters and Parameter Control Studies}

While not essential to our conclusions, we nevertheless sought to use appropriate physiological and anatomical data to constrain model design and associated parameters. These were obtained from the non-human primate literature and our own experience with macaque V2. The size and distributions of the stripes and functional domains in V2, and their variance, have been qualitatively described by others using histological and functional imaging approaches. Our domain shape and size metrics and stripe width and functional organization characteristics are idealized but are generally consistent with these data from macaque V2 (Olavarria and Van Essen, 1997; Ts'o et al., 2001; Xiao et al., 2003; Lu and Roe, 2008; Ts'o et al., 2009). While it is feasible to generate model maps from actual functional topographies (Grinvald et al., 1986; 
Swindale, 2007), we felt that it was appropriate here to simplify the shapes and organizations for programming efficiency and to allow objective comparisons. Our idealized circular or elongated domain forms in Model 1 could then be expanded in breadth or varied in separation in highly controlled manners.

The overall densities of model cells, terminals and spines are comparable to published experimental reports (cf. (Elston and Rosa, 1997; Markram et al., 1997; Elston et al., 1999; Stepanyants et al., 2002; Feldmeyer et al., 2006)). Quantitative details of the typical number of terminal boutons per cell in primate V2, and their relative proportional distributions to immediate radial or nearby clustered locations (and other areas) are still not known. Although the proportional extent of neuronal preference within a given V2 domain is still unknown, recent data using in vivo 2-photon imaging suggests that functional domains in early visual cortical areas comprise very high proportions of neurons that share a common preference (Ohki et al., 2005). Our control parameter manipulation studies (see Figure 3-8) however indicate that precise knowledge of actual terminal and spine count per cell in V2, and of overall relative contribution of intra-areal (vs. inter-areal) inputs, and of domain purity extents are not essential to ensure our main results. Injected tracer studies qualititatively indicate that V2 cortical neurons do project a substantial proportion of their axons locally within the immediate radial vicinity of the cell body, with a relatively high density typically extending 400-600 $\mu$ m (Amir et al., 1993). In addition more spatially extensive and clustered projections extend up to $4 \mathrm{~mm}$, with typical distance between clusters ranging $700 \mu \mathrm{m}$ to $2 \mathrm{~mm}$ (Amir et al., 1993; Malach et al., 1993; Angelucci et al., 2002). For simplicity of implementation and to permit controlled specification and data interpretation we used a homogeneous radial exponential falloff (Sholl, 1953; Abeles, 1991). A spatial decay constant of $100 \mu \mathrm{m}$ for terminals yielded a conservative radial influence (cf. Gaussian or anisotropic model distributions), and therefore may underestimate the radial extent of functional joint probability that may be supported by intrinsic clusters and more specific (nonrandom) circuitry (Song et al., 2005). The homogenous spatial decay is consistent with the idealized surround distribution used in many cortical network models (Stemmler et al., 1995; Spiridon and Gerstner, 2001; Kang et al., 2003). Others have used connection probabilities that are functions of orientation difference, consistent with connection reports in primary visual cortex where like-with-like projections occur (cf. (Koulakov and Chklovskii, 2001)). We did not apply an orientation dependent rule in our model because the phenomenon is less prevalent in V2 
(Malach et al., 1993; Tamura et al., 1996). Results from our control experiments that introduced V2-like patchy projections as progressively increasing proportions of the overall terminal distributions do not suggest a change to our primary conclusions.

Joint probabilities remained significantly elevated compared with random control data, up to at least $75 \%$ patchy projection proportion. Nevertheless, we note that majority proportions of patchy projections could reduce $\mathrm{P}_{\mathrm{OC}-\mathrm{ROI}}$ between paired domains (by about 25\% at $75 \%$ patchy proportion) and increase variance. Further detailed experiments that systematically investigate a range of biologically-motivated patchy projection configurations and their influence on joint probabilities would further usefully elaborate these differential organizational issues.

We also sought to use biologically inspired information to guide our Model 2 configurations, while simplifying organizational aspects to enable sufficiently controlled interpretation. It should be noted that the appropriate number of domain categories used in our model maps is quite subjective. Actual cortical organization is not likely to be a mosaic of distinct domains with specific preferences, but rather is thought to be a mapped continuum of systematic orientations and color progressions. Thus the number of categories chosen here represents a categorical sampling of the continuum. A larger number of categories to be mapped at fixed spacing would be expected to yield a larger surface area in which a full cycle of preferences can be mapped ('hypercolumn’). Xiao and Felleman (Xiao et al., 2003) reported full cycles of hue representation in thin stripes in discrete 'modules' of $\leq 1 \mathrm{~mm}^{2}$ of V2 topography. These often appeared as modular islands separated by other luminance modules. Roe and colleagues (Roe and Ts'o, 1995; Ts'o et al., 2001; Lu and Roe, 2008) also described similar topographic coverage for color and orientation hypercolumns. Our packing of four categories packed at 400 or $500 \mu \mathrm{m}$ spacing is probably more consistent with these findings than eight categories. Reconstructed representations of optical image maps that would be represented by our packings were made (data not shown) and map reconstructions based on four rather than eight categories appeared more consistent with those color modules experimentally recorded by Felleman and colleagues (Xiao et al., 2003).

\subsubsection{Domain Organization and Intra-Areal Integration of Function as a General Principle}

The neocortex is a highly evolved complex dynamical system with recurrent interconnections and interactions likely to occur at many spatial and temporal scales (Edelman, 
1987; Felleman and Van Essen, 1991). Convergence and divergence between, rather than within, cortical areas are therefore likely to remain a dominant constructive phenomenon, and this may be associated with an optimal cluster size. It is known that neurons that are close together are more likely to (at least weakly) fire together (Douglas et al., 1995; Constantinidis and GoldmanRakic, 2002) and this pooling of neural response by neighboring populations may theoretically improve signal to noise ratio of processing (of a common response property) in proportion to population size (Zohary et al., 1994). Our modeling, however, suggests that functional clustering within cortex may provide a geometric framework that may also assist in the combination of different functional inputs in some areas. This may be especially so in higher order sensory areas such as Areas V2 and V4, and possibly infero-temporal cortex, where (i) domains are relatively large, (ii) neighboring domains can map substantially different sensory phenomenon, (iii) intrinsic outputs are more extensive and less specific (cf. like-to- like connectivity well described in V1), and (iv) single neurons share selectivities in common with their domains, supplemented by additional selectivities to other unique attributes (different from their domain) (Amir et al., 1993; Malach et al., 1994; Tamura et al., 1996; Ghose and Ts'o, 1997; Sato et al., 2009).

\subsubsection{Conclusions and Future Experiments}

These results suggest that the clustering of neurons with similar preference offers potential advantages for some classes of neuronal computations. Using a model based on current knowledge of early visual cortical organization and a simple radially diffuse (cf. patchy) terminal distribution, we have shown that the domain and stripe organization of primate V2 may feasibly

offer a well-tailored substrate for enhanced integration of output from groups of visually responsive neurons with quite different stimulus preferences. The data support the possibility of a particular selection of combination (color and orientation) although other combinations associated with different domain class preferences are equally feasible. This combination could occur via two different computational mechanisms: intra-areal domain-mediated convergence (enhanced probability of spatial correlation of color- preferring and orientation-preferring terminals), and intra-areal domain-mediated modulation (enhanced probability of spatial correlation of color-preferring terminal and orientation-preferring spines, and vice versa).

Although V2 is already a well-studied cortical area, there is still very little quantitative data regarding the relative contributions of inter-areal vs. intra-areal circuits in V2 and their 
associated spine and terminal frequency and stimulus preference. Moreover, the contributory role of blob and interblob output (and their precise functional correlates) onto the different V2 stripes also needs further investigation and clarification. Further electrophysiological, neuroanatomical, and histological experiments that may elaborate these contributions would be helpful for future modeling work. Recent models have begun to elicit how competitive developmental mechanisms may give rise to specific V2 functional organizations (Swindale, 2007). In future work, our model may be usefully extended by further incorporating the representations of patchy intra-areal and inter-areal connectivities, as we learn how patchy projections associate with the underlying functional map (Shmuel et al., 2005; Shipp et al., 2009; Shushruth et al., 2009). Finally, our model findings suggest future in vivo experiments that specifically investigate the role that intraareal inter-domain projections may play in visual processing. We suggest that non human primate V2 in particular is an ideal cortical area to investigate these mechanisms. Relative to other visual areas such as V1, Area V2 offers large and readily identified and neighboring coloror orientation-preferring domains which can be readily sampled using conventional neurophysiological techniques guided by functional imaging techniques. That these domains appear to prefer color (or orientation) while exhibiting random organization for orientation (or color) could prove especially useful for highly controlled but functionally selective domain inactivation experimental designs. 


\subsection{References}

Abeles M (1991) Corticonics: Neural Circuits of the Cerebral Cortex. Cambridge: Cambridge University Press.

Amir Y, Harel M, Malach R (1993) Cortical hierarchy reflected in the organization of intrinsic connections in macaque monkey visual cortex. J Comp Neurol 334:19-46.

Angelucci A, Levitt JB, Walton EJ, Hupe JM, Bullier J, Lund JS (2002) Circuits for local and global signal integration in primary visual cortex. J Neurosci 22:8633-8646.

Binzegger T, Douglas RJ, Martin KA (2004) A quantitative map of the circuit of cat primary visual cortex. J Neurosci 24:8441-8453.

Callaway EM (1998) Local circuits in primary visual cortex of the macaque monkey. Annu Rev Neurosci 21:47-74.

Chen BL, Hall DH, Chklovskii DB (2006) Wiring optimization can relate neuronal structure and function. Proc Natl Acad Sci U S A 103:4723-4728.

Chen LM, Turner GH, Friedman RM, Zhang N, Gore JC, Roe AW, Avison MJ (2007) Highresolution maps of real and illusory tactile activation in primary somatosensory cortex in individual monkeys with functional magnetic resonance imaging and optical imaging. $\mathrm{J}$ Neurosci 27:9181-9191.

Constantinidis C, Goldman-Rakic PS (2002) Correlated discharges among putative pyramidal neurons and interneurons in the primate prefrontal cortex. J Neurophysiol 88:3487-3497.

Douglas RJ, Koch C, Mahowald M, Martin KA, Suarez HH (1995) Recurrent excitation in neocortical circuits. Science 269:981-985.

Edelman GM (1987) Neural Darwinism: The Theory of Neuronal Group Selection. New York: Basic Books.

Elston GN, Rosa MG (1997) The occipitoparietal pathway of the macaque monkey: comparison of pyramidal cell morphology in layer III of functionally related cortical visual areas. Cereb Cortex 7:432-452.

Elston GN, Tweedale R, Rosa MG (1999) Cortical integration in the visual system of the macaque monkey: large-scale morphological differences in the pyramidal neurons in the occipital, parietal and temporal lobes. Proc Biol Sci 266:1367-1374.

Feldmeyer D, Lubke J, Sakmann B (2006) Efficacy and connectivity of intracolumnar pairs of layer 2/3 pyramidal cells in the barrel cortex of juvenile rats. J Physiol 575:583-602. 
Felleman DJ, Van Essen DC (1991) Distributed hierarchical processing in the primate cerebral cortex. Cereb Cortex 1:1-47.

Friedman HS, Zhou H, von der Heydt R (2003) The coding of uniform colour figures in monkey visual cortex. J Physiol 548:593-613.

Gegenfurtner KR, Kiper DC, Fenstemaker SB (1996) Processing of color, form, and motion in macaque area V2. Vis Neurosci 13:161-172.

Ghose GM, Ts'o DY (1997) Form processing modules in primate area V4. J Neurophysiol 77:2191-2196.

Gilbert CD (1983) Microcircuitry of the visual cortex. Annu Rev Neurosci 6:217-247.

Grinvald A, Lieke E, Frostig RD, Gilbert CD, Wiesel TN (1986) Functional architecture of cortex revealed by optical imaging of intrinsic signals. Nature 324:361-364.

Horton JC, Adams DL (2005) The cortical column: a structure without a function. Philos Trans R Soc Lond B Biol Sci 360:837-862.

Hubel DH, Wiesel TN (1962) Receptive fields, binocular interaction and functional architecture in the cat's visual cortex. J Physiol 160:106-154.

Hubel DH, Wiesel TN (1969) Anatomical demonstration of columns in the monkey striate cortex. Nature 221:747-750.

Hubel DH, Livingstone MS (1987) Segregation of form, color, and stereopsis in primate area 18. J Neurosci 7:3378-3415.

Johnson EN, Hawken MJ, Shapley R (2001) The spatial transformation of color in the primary visual cortex of the macaque monkey. Nat Neurosci 4:409-416.

Kang K, Shelley M, Sompolinsky H (2003) Mexican hats and pinwheels in visual cortex. Proc Natl Acad Sci U S A 100:2848-2853.

Kim DS, Duong TQ, Kim SG (2000) High-resolution mapping of iso-orientation columns by fMRI. Nat Neurosci 3:164-169.

Kiper DC, Fenstemaker SB, Gegenfurtner KR (1997) Chromatic properties of neurons in macaque area V2. Vis Neurosci 14:1061-1072.

Kohonen T (1982) Self-organized formation of topologically correct feature maps. Biological Cybernetics 43:59-69.

Koulakov AA, Chklovskii DB (2001) Orientation preference patterns in mammalian visual cortex: a wire length minimization approach. Neuron 29:519-527. 
Landisman CE, Ts'o DY (2002a) Color processing in macaque striate cortex: relationships to ocular dominance, cytochrome oxidase, and orientation. J Neurophysiol 87:3126-3137.

Landisman CE, Ts'o DY (2002b) Color processing in macaque striate cortex: electrophysiological properties. J Neurophysiol 87:3138-3151.

Levitt JB, Yoshioka T, Lund JS (1994a) Intrinsic cortical connections in macaque visual area V2: evidence for interaction between different functional streams. J Comp Neurol 342:551-570.

Levitt JB, Kiper DC, Movshon JA (1994b) Receptive fields and functional architecture of macaque V2. J Neurophysiol 71:2517-2542.

Livingstone MS, Hubel DH (1984) Anatomy and physiology of a color system in the primate visual cortex. J Neurosci 4:309-356.

Lu HD, Roe AW (2008) Functional organization of color domains in V1 and V2 of macaque monkey revealed by optical imaging. Cereb Cortex 18:516-533.

Lund JS, Yoshioka T, Levitt JB (1993) Comparison of intrinsic connectivity in different areas of macaque monkey cerebral cortex. Cereb Cortex 3:148-162.

Malach R, Tootell RB, Malonek D (1994) Relationship between orientation domains, cytochrome oxidase stripes, and intrinsic horizontal connections in squirrel monkey area V2. Cereb Cortex 4:151-165.

Malach R, Amir Y, Harel M, Grinvald A (1993) Relationship between intrinsic connections and functional architecture revealed by optical imaging and in vivo targeted biocytin injections in primate striate cortex. Proc Natl Acad Sci U S A 90:10469-10473.

Markram H, Lubke J, Frotscher M, Roth A, Sakmann B (1997) Physiology and anatomy of synaptic connections between thick tufted pyramidal neurones in the developing rat neocortex. J Physiol 500 (Pt 2):409-440.

Mountcastle VB, Davies PW, Berman AL (1957) Response properties of neurons of cat's somatic sensory cortex to peripheral stimuli. J Neurophysiol 20:374-407.

Ohki K, Chung S, Ch'ng YH, Kara P, Reid RC (2005) Functional imaging with cellular resolution reveals precise micro-architecture in visual cortex. Nature 433:597-603.

Olavarria JF, Van Essen DC (1997) The global pattern of cytochrome oxidase stripes in visual area V2 of the macaque monkey. Cereb Cortex 7:395-404.

Peters A, Sethares C (1996) Myelinated axons and the pyramidal cell modules in monkey primary visual cortex. J Comp Neurol 365:232-255. 
Piepenbrock C, Obermayer K (2000) The effect of intracortical competition on the formation of topographic maps in models of Hebbian learning. Biol Cybern 82:345-353.

Ramsden BM, Hung CP, Roe AW (2001) Real and illusory contour processing in area V1 of the primate: a cortical balancing act. Cereb Cortex 11:648-665.

Roe AW, Ts'o DY (1995) Visual topography in primate V2: multiple representation across functional stripes. J Neurosci 15:3689-3715.

Roe AW, Ts'o DY (1999) Specificity of color connectivity between primate V1 and V2. J Neurophysiol 82:2719-2730.

Sato T, Uchida G, Tanifuji M (2009) Cortical columnar organization is reconsidered in inferior temporal cortex. Cereb Cortex 19:1870-1888.

Shipp S, Zeki S (2002) The functional organization of area V2, I: specialization across stripes and layers. Vis Neurosci 19:187-210.

Shipp S, Adams DL, Moutoussis K, Zeki S (2009) Feature binding in the feedback layers of area V2. Cereb Cortex 19:2230-2239.

Shmuel A, Korman M, Sterkin A, Harel M, Ullman S, Malach R, Grinvald A (2005) Retinotopic axis specificity and selective clustering of feedback projections from V2 to V1 in the owl monkey. J Neurosci 25:2117-2131.

Sholl DA (1953) The Organization of the Cerebral Cortex. London: Methuen and Co.

Shushruth S, Ichida JM, Levitt JB, Angelucci A (2009) Comparison of spatial summation properties of neurons in macaque V1 and V2. J Neurophysiol:(in press).

Sincich LC, Horton JC (2002) Divided by cytochrome oxidase: a map of the projections from V1 to V2 in macaques. Science 295:1734-1737.

Song S, Sjostrom PJ, Reigl M, Nelson S, Chklovskii DB (2005) Highly nonrandom features of synaptic connectivity in local cortical circuits. PLoS Biol 3:e68.

Spiridon M, Gerstner W (2001) Effect of lateral connections on the accuracy of the population code for a network of spiking neurons. Network 12:409-421.

Stemmler M, Usher M, Niebur E (1995) Lateral interactions in primary visual cortex: a model bridging physiology and psychophysics. Science 269:1877-1880.

Stepanyants A, Chklovskii DB (2005) Neurogeometry and potential synaptic connectivity. Trends Neurosci 28:387-394. 
Stepanyants A, Hof PR, Chklovskii DB (2002) Geometry and structural plasticity of synaptic connectivity. Neuron 34:275-288.

Swindale NV (2007) A model for the thick, thin and pale stripe organization of primate V2. Network 18:327-342.

Swindale NV, Shoham D, Grinvald A, Bonhoeffer T, Hubener M (2000) Visual cortex maps are optimized for uniform coverage. Nat Neurosci 3:822-826.

Szentagothai J (1978) The Ferrier Lecture, 1977. The neuron network of the cerebral cortex: a functional interpretation. Proc R Soc Lond B Biol Sci 201:219-248.

Tamura H, Sato H, Katsuyama N, Hata Y, Tsumoto T (1996) Less segregated processing of visual information in V2 than in V1 of the monkey visual cortex. Eur J Neurosci 8:300309.

Tootell RB, Hamilton SL (1989) Functional anatomy of the second visual area (V2) in the macaque. J Neurosci 9:2620-2644.

Ts'o DY, Roe AW, Gilbert CD (2001) A hierarchy of the functional organization for color, form and disparity in primate visual area V2. Vision Res 41:1333-1349.

Ts'o DY, Zarella M, Burkitt G (2009) Whither the hypercolumn? J Physiol 587:2791-2805.

Vanduffel W, Tootell RB, Schoups AA, Orban GA (2002) The organization of orientation selectivity throughout macaque visual cortex. Cereb Cortex 12:647-662.

Wang Y, Xiao Y, Felleman DJ (2007) V2 thin stripes contain spatially organized representations of achromatic luminance change. Cereb Cortex 17:116-129.

Xiao Y, Felleman DJ (2004) Projections from primary visual cortex to cytochrome oxidase thin stripes and interstripes of macaque visual area 2. Proc Natl Acad Sci U S A 101:71477151.

Xiao Y, Zych A, Felleman DJ (1999) Segregation and convergence of functionally defined V2 thin stripe and interstripe compartment projections to area V4 of macaques. Cereb Cortex 9:792-804.

Xiao Y, Wang Y, Felleman DJ (2003) A spatially organized representation of colour in macaque cortical area V2. Nature 421:535-539.

Yacoub E, Harel N, Ugurbil K (2008) High-field fMRI unveils orientation columns in humans. Proc Natl Acad Sci U S A 105:10607-10612.

Zhaoping L (2005) Border ownership from intracortical interactions in visual area v2. Neuron 47:143-153. 
Zohary E, Shadlen MN, Newsome WT (1994) Correlated neuronal discharge rate and its implications for psychophysical performance. Nature 370:140-143. 
CHAPTER 4: Sensory integration and temporal response modulation in macaque Area V2: Single Unit Electrophysiology 


\subsection{Abstract}

Neurons in the second visual cortical area (Area V2) of macaque monkeys organize according to response preferences of specific color, orientation or luminance stimuli. This clustering potentially influences V2 neuronal activity such as temporal response spiking characteristics, but the effect is not well documented. To investigate the temporal changes in V2 neurons' spiking activity associated with the controlled addition of color to an oriented achromatic stimulus condition, we collected in vivo single unit electrophysiological recordings in anesthetized macaques. Using optical imaging difference maps as guides and cytochrome oxidase as verification, orientation selective V2 cells were identified and presented with 1) luminance-controlled, achromatic, preferred-orientation and 2) preferred-color, preferredorientation stimuli during single unit electrophysiological recordings. The temporal responses of stimulus-evoked spike firing were categorized, normalized and plotted in two-dimensional summary profile matrices. Using spike density function profiles and threshold criteria on these profiles, we calculated latencies and point of maximum spiking rate of each cell in response to the achromatic and colored stimuli. Our data suggest that the addition of a color to an achromatic ('uncolored') orientated stimulus produces significant increases in mean latency, significantly increases mean time of peak firing rate, and generates a significant reduction in the initial, transient phase but preserves the later, sustained spiking response. These relative changes in temporal patterns of spiking activity cannot be simply explained by the known properties of ascending cortical inputs and pathways. In V2, color-preferring and orientation-preferring neurons are systematically organized into neighboring (and richly interconnected) large scale functional domains. We suggest that the stimulus evoked changes in temporal activity response reported here may be supported by a mechanism of complex intra-cortical interactions associated with these functional organizations of V2. 


\subsection{Introduction}

Research within the second visual area (V2) in Old World macaque monkeys has demonstrated a high degree of cortical organization, with V2 neurons seemingly arranged according to a preference for at least one visual modality, such as orientation or color. A repeating, stripe-like pattern encompassing the entirety of V2 is evident when stained with the mitochondrial enzyme cytochrome oxidase (CO) (Wong-Riley, 1979). The resulting design consists of a repeating thick-pale-thin-pale design, where a single cycle spans $\sim 4 \mathrm{~mm}$ of cortex (Tootell and Hamilton, 1989) and a dozen cycles cover the length of V2 (Livingstone and Hubel, 1983; Horton, 1984; Hubel and Livingstone, 1987; Roe and Ts'o, 1995; Olavarria and Van Essen, 1997; Sincich and Horton, 2003). The thick and thin stripes consist of "thick" or "thin" CO-dark bands, respectively, while pale stripes are bands of markedly little CO staining. The neurons within thin stripes are typically sensitive, or "tuned”, to the surface properties of a visual stimulus - such as color or luminance - while thick and pale stripes are usually reported to be organized according to the orientation of the stimulus, although other more complex attributes associated with "form” have also been reported (e.g. v-shaped corners and higher order contours) (Tootell and Hamilton, 1989; Levitt et al., 1994a; Roe and Ts'o, 1995; Ts'o et al., 2001; Vanduffel et al., 2002; Xiao et al., 2003; Wang et al., 2006).

In contrast to V2, CO-staining in Area V1 (primary or striate visual cortex) typically manifests as ovaloid shapes of 150-200 $\mu \mathrm{m}$ breadth, variously termed "blobs" or "puffs" rather than stripes. These have been documented using electrophysiological methods as containing neurons that increase their firing rates according to color-preference while the neurons in nonstained, “inter-blob” (“inter-puff”) region exhibit increased spike firing according to orientation preferences. These color and orientation compartments in V1 are thought to remain predominantly segregated as they project as inputs into V2: blobs transmit color information to thin stripes while inter-blob inputs send orientation to pale stripes (Livingstone and Hubel, 1984; Hubel and Livingstone, 1987; Sincich and Horton, 2002). Intrinsic signaling imaging studies confirm the preferential activity of these discrete blob-like areas in awake, behaving monkeys (Vnek et al., 1999), and the parallel processing of visual information (e.g. color, orientation, motion), along with the anatomical observations described here, such as the CO staining regions in V1 and V2, corroborate these studies. 
Early observations noted a pattern of like to like connectivity V1 blob compartments tended to interconnect more with blobs rather than interblobs. Nevertheless, other studies have suggested that the 'parallel' streams are not completely segregated: some communications have been documented between the blob and interblob compartments of V1 (Lachica et al., 1992; Yoshioka et al., 1994) and the various stripes of V2 (Levitt et al., 1994b), and the projections from V1 onto V2 have also been reported to terminate in a more diverse cortical area than original purported. For example, both inter-blob regions and blobs project to V2 thin stripes (Xiao and Felleman, 2004). Short-ranged connections between neurons within the same cortical region, termed intra-areal or "intrinsic" connections, and the effects that these connections may have on surrounding cells have been documented in V1 in cats (Gilbert and Wiesel, 1990) and macaque monkeys (Yoshioka et al., 1996). Such intra-areal connections have also been documented between and among the stripes of V2 (Levitt et al., 1994b; Kiper et al., 1997; Shipp and Zeki, 2002); the close proximity of these stripes is within the estimated $400 \mu \mathrm{m}$ projection distance reportedly necessary for neurons of one domain to influence neurons of a neighboring domain (Malach et al., 1993). Thus, the resulting rich intra-areal connectivity could feasibly modulate V2 response properties through mechanisms of excitation or inhibition, leading to a diverse combination of various preference and tuning characteristics.

A notable aspect of functional organization in V2 is that neurons organize into different functional "domains" according to stripe: color-preferring domains are typically found in thin stripes, while orientation-preferring domains are usually located in the pale and thick stripes. Other domain types (e.g. disparity) have also been reported in V2. This dichotomous color/orientation pattern of stripe organization has been confirmed by optical imaging experiments (Figure 4-1), but these domain descriptions within stripes pertain to the population of cells; Importantly, an individual cell, regardless of stripe or domain location, can nevertheless have both a color and orientation preference (Tamura et al., 1996; Gegenfurtner and Kiper, 2003).

If intra-areal connections modulate the temporal spiking activity of a cell's response (Sporns et al., 2000; Fries et al., 2001; Knoblauch and Palm, 2002), these modulations could be perceived through changes in latency (time from stimulus presentation to neuronal spiking response) when a single neuron is presented with differing stimuli. Previous reports found that V2 latencies do depend on stimulus attributes to some extent: color-preferring cells displayed a 
relatively long latency $(93.3 \pm 5.7 \mathrm{msec})$ as opposed to shorter latencies $(78.1 \pm 2.4 \mathrm{msec}$ and $74.6 \pm 1.7$ msec for oriented- and achromatic-preferring (non-color selective) cells respectively (Munk et al., 1995). Stripe location also plays a role in a cell's latency: thin stripe cells (potentially organized in color-preferring domains), yielded longer latencies (93.5 $\pm 5.8 \mathrm{msec}$ ) while cells in pale or thick stripes (potentially organized in orientation-preferring domains) yielded shorter latencies (72.9 $\pm 5.5 \mathrm{msec}$ and $76.2 \pm 3.6 \mathrm{msec}$ ) respectively (Munk et al., 1995).
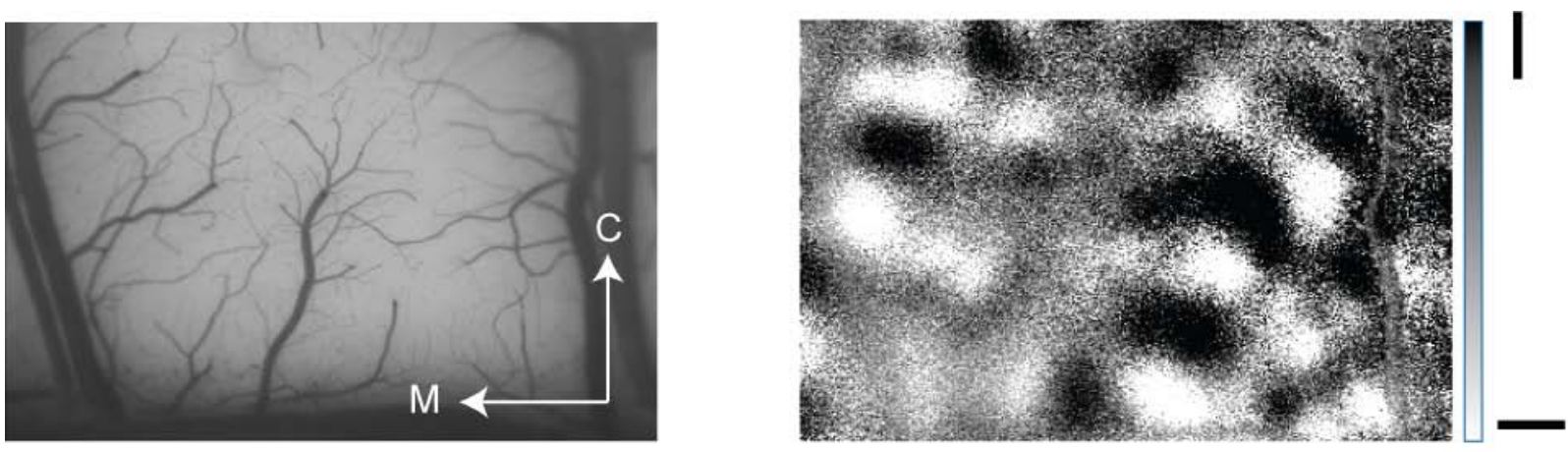

$1 \mathrm{~mm}$
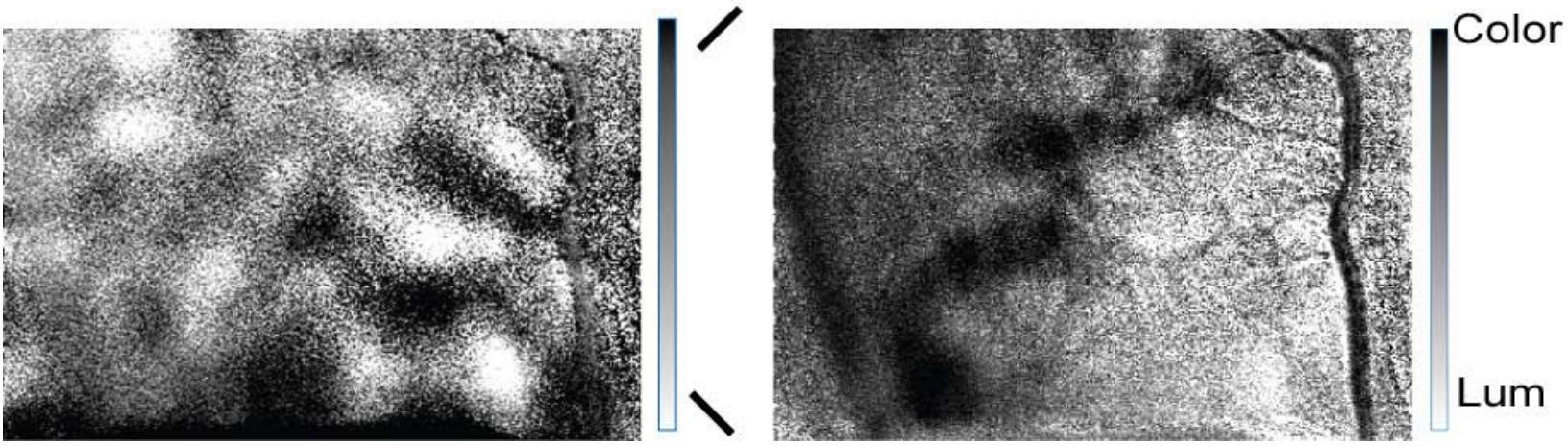

Figure 4-1. Intrinsic signal optical imaged difference maps. (A) The in vivo vessel pattern of the visual cortex of a representative macaque, positioned over the V2 area to guide electrode penetrations. The image is aligned such that the lunate sulcus runs along the bottom; the caudal region of the cortex is at the top of the image and the medial towards the left. (B) Corresponding horizontal-vertical difference map for orientation, (C) oblique-acute and (D) color-luminance -preference. Using these maps in tandem, it is possible to ascertain orientation- and colorpreferring domains such as the pale/thick and thin stripes. Electrode penetrations are guided by these maps to ensure locations are within the V2 region. Scale bar: $1 \mathrm{~mm}$. C: caudal. M: medial.

This discrepancy associated with stripe location has further been attributed to distinct input pathways from V1 onto V2 (Schmolesky et al., 1998), and may be consistent with the notion of largely segregated blob to thin and inter-blob to pale/thick inter-areal projections (Sincich \& Horton, 2002). These reports, however, did not always examine the effects of the same singular 
neuron to various distinct or combined stimulus attributes (e.g., chromatic vs. achromatic oriented bars) in order to systematically compare the resulting changes in temporal responses. Axonal conduction studies purport that information traveling along the magnocellular pathway (achromatic and motion information) from the lateral geniculate nucleus reaches cortical areas significantly faster than signals sent along the parvocellular pathway (color and higher resolution acuity information) (Maunsell et al., 1999). Thus, an achromatic luminant bar stimulus (e.g. bar foreground and bar background have different luminance but exhibit no color) might be expected to yield a shorter latency in a V2 neuron than an colored but isoluminant bar stimulus (i.e. bar foreground and bar background have different color, but same luminance) presented to the same cell. Mixing of parvo- and magnocellular streams in V1 does occur, so a combination of color, orientation and luminance in a singular stimulus could evoke a latency equivalent to the achromatic stimulus (the fastest component). A proposed third pathway, the koniocellular pathway, sends projections of S-cone inputs to the cytochrome oxidase blobs in V1, and these inputs have been suggested to arrive relatively late in V1 - 96 to $135 \mathrm{msec}$ (Cottaris and De Valois, 1998; Casagrande, 1999; Hendry and Reid, 2000). Some of the earliest studies used light and dark bars of optimal length and orientation to report mean latency times of 85ms in V1 and 96ms in V2. However, only a relatively few number of cells were observed (Raiguel et al., 1989).

Previous perceptual psychophysical studies have investigated a similar question concerning the affect of a stimulus' color on latency by examining the effects of color/achromatic or 'white' light wavelength on reaction time (RT). When presented on a dimmer, achromatic background RT did not vary with equiluminant wavelength (color wavelengths or white light) stimulation, however when the background luminance matched that of the stimuli presented, variations in RT were observed (Holmes, 1926; Lit et al., 1971; Mollon and Krauskopf, 1973; Nissen and Pokorny, 1977). Stimuli along the blue-yellow (S-[L+M]) axis yielded a longer RT than those along the red-green (L-M) opponent axis (McKeefry et al., 2003), suggesting that a chromatic cue and not a luminance one affect the respondent's RT.

We investigated the experimental evidence that these properties can be differentially characterized through the selective modulation of a single neuron's temporal activity when presented with two different stimuli, such as an achromatic- vs. a colored-oriented bar. We recorded from single neurons in $\mathrm{V} 2$ in a series of in vivo experiments using anesthetized 
macaque monkeys. By applying intrinsic signal optical imaging as a guide for single unit electrophysiology studies, we determined the temporal response of V2 neurons to an achromatic, preferred-orientation stimulus condition, and also to a preferred color, preferred-orientation combined stimulus condition. This method allowed us to discern the possible differential effect that two different stimuli can have on a single cell's temporal spiking activity profile. We found that both latency and maximum firing time lengthen with the addition of color to the oriented stimulus and furthermore, we observed that there is a significant dampening of the initial temporal response while the sustained response remains unmodulated. 


\subsection{Methods}

\subsubsection{Experimental Animals:}

Three young adult female Macaca fascicularis, obtained from approved suppliers, were anesthetized (sufentanil citrate, $4-12 \mu \mathrm{g} / \mathrm{kg} / \mathrm{hr}$ and isoflurane, $0.25-1.5 \%$ ) and paralyzed (vercuronium bromide, $50 \mu \mathrm{g} / \mathrm{kg} / \mathrm{hr}$ ). Animals were artificially ventilated while monitoring anesthetic depth through EEG, EKG, respiration rate with end-tidal $\mathrm{CO}_{2}$, oximetry, blood pressure, and pulse-based heart-rate monitoring (Cardell MAX-1, Sharn Veterinary, Inc., Tampa, FL). Frequent response-tests to toe/tail pinch were also used to note any changes in physiological state. Animals' eyelids were retracted with specula and the pupils dilated with topical atropine drops. Research grade primate contact lenses (Danker Laboratories Inc., Sarasota, FL) were placed over each of the eyes to protect the corneas and to direct animal's visual focus onto the computer monitor (Barco Calibrator VCID-421, Belgium) positioned 57 inches away. Retinotopic location of each optic disc and eye refraction were determined with an ophthalmoscope to ensure the optical focal length. A 7/8” diameter circular craniotomy and subsequent durotomy under sterile, aseptic surgical conditions were performed 5-15 mm lateral of the midline cranial suture and 14-20 mm rostral of the occipital cranial ridge - approximately over the lunate sulcus. A nylon chronic chamber was placed into the newly formed aperture, allowing a single animal to be utilized over several procedures (Chen et al., 2002). All experiments were performed under approved protocols sanctioned by West Virginia Animal Care and Use Committee and Office of Laboratory Animal Resources.

\subsubsection{Visual stimuli:}

A custom-made Delphi language program generated the visual stimuli of achromatic (white) orientation bars (ABar) and monochrome colored (non-white) orientation bars (CBar) on a CRT monitor (Barco Calibrator V, Model VCID-421, Belgium). Stimulus presentation always consisted of a 200 msec display of the orientation bar immediately followed by a 200 msec blank screen.

ABars consisted of a computer generated linear rectangular elements with cell-preferred pixel widths (2-3 minutes of arc) and preferred lengths (1-3 degrees visual angle). Preferred widths and lengths were checked and determined by manual control of computer generated stimulus generation. The presented sequence of orientations ranged from $0^{\circ}$ (a horizontal line) 
and increased at $11.25^{\circ}$ intervals up to $168.75^{\circ}$; with the order of presentation of each orientation randomly determined. Stimuli were displayed at $37 \mathrm{~cd} / \mathrm{m}^{2}$ on a $6 \mathrm{~cd} / \mathrm{m}^{2}$ darkened screen. CBar stimuli consisted of a cell's ABar preference (i.e. preferred orientation, optimal width and optimal length at $37 \mathrm{~cd} / \mathrm{m}^{2}$ ) displayed in one of 12 possible isoluminant “colors” (teal, cyan, blue, blue-violet, violet, magenta, pink, orange, orange-yellow, yellow, lime, green, and white-point) equally distributed across the Derrington-Lennie-Krauskopf (DLK) color space (Derrington et al., 1984). The CBar stimulus set was presented in a pseudo-random manner with pairs of interleaving randomly selected stimuli to prevent potential complications in response collection and interpretation from adaptation. Aside from the addition of color, all other CBar stimulus variables (e.g., orientation angle, luminance) were controlled and were identical to the corresponding ABar variables.

\subsubsection{Intrinsic signal optical imaging:}

Functional organization maps of visual response in V2 using in vivo video recordings of intrinsic signals at $605(+/-10) \mathrm{nm}$ helped guide electrode placement and identify important organization sub-compartments within V2 (Figure 4-1). The intrinsic optical imaging can monitor the conversion of oxyhemoglobin to deoxyhemoglobin in the microcirculation of the cortex, and this is associated with increased neuronal activity. Acquisition and averaging of video image frames allows the generation of two dimensional maps of the increased activities associated with specific stimulus condition presentations. The optimum proposed wavelength of 600nm indicates changes in oxygen state (Grinvald et al., 1986; Frostig et al., 1990). The imaging camera lens system was positioned over the lunate sulcus at the boundary of the occipital lobe. We used difference mapping methods to subtract left eye responses from right eye responses to obtain a functional “ocular dominance” map. We used the observed presence of ocular dominance columns, indicative of Area V1 (ocular dominance columns are absent in V2), to functionally locate the V1/V2 border.

\subsubsection{Single unit electrophysiology:}

Recordings were made using fine, glass-coated (Micro Probe, Inc., Gaithersburg, MD) or

parylene-coated (Nano Bio Sensors, We Sense Ltd., Nazareth, Israel) tungsten electrodes inserted into upper layers of the V2 cortex (0 - $2 \mathrm{~mm}$ deep). Small increments of 5-10 $\mu \mathrm{m}$ were 
traversed down through the layers of cortex to isolate a single, responsive neuron. Only units that could be clearly isolated, adequately and robustly “driven” with visual stimulation, and displayed significant selectivity for orientation were chosen for a more intensive quantification study of orientation and/or color preference, as determined by readings from an oscilloscope (GRS6032A, Instek, Chino, CA) and audio monitoring. Data acquisition was performed using Spike 2 on Power 1401 (version 6.10, Cambridge Electronic Design, Cambridge UK), which was stored on the hard drive then analyzed offline.

\subsubsection{Histology:}

Each monkey underwent a sequence of chronic recovery experiments completed by a terminal experiment in which electrode lesions $(7 \mu \mathrm{A}, 7 \mathrm{~s})$ were made to aid in tissue realignment. An overdose of sodium pentobarbital $(116 \mathrm{mg} / \mathrm{kg})$ was given intravenously and we then perfused intracardially with physiological saline rinse followed by a $3 \%$ buffered paraformaldehyde fixative. The brain was then removed, sectioned parallel to the V1/V2 border, and the sections sunk in 30\% sucrose. The tissue was prepared into 30-50 micron slices parallel to the cortical surface and prepared for histological processing with cytochrome oxidase. The slices were aligned post-hoc in Adobe Photoshop (Adobe Systems Incorporated, San Jose, CA) according to cytological and surface vessel landmarks and electrode lesions. The histological data, much like penetration location on the intrinsic imaging map, further provide an anatomical correlation of a neuron's stimulus preferences and spiking characteristics and corroborated V1/V2 border location.

\subsubsection{Preference and Latency Determination:}

A spike density function (SDF) graph of spikes per unit time (Szucs, 1998) was created to examine the temporal properties of a cell's response to the stimuli. A baseline noise value was calculated from a representative region of the recording (e.g. the 'blank' period of stimulus presentation) (Priebe et al., 2006). The threshold for significant peaks of activity was calculated as two standard deviations (of baseline variance) greater than the baseline (Smith et al., 2006) (Figure 4-2A). A cell's "preferred stimulus" was defined as the orientation angle or color displaying the greatest total cellular activity above baseline (i.e. noise). For a cell to be further 
examined, the preferred color could be any hue except achromatic (white). Both "raw" (unmodified count) and "smooth" counts (the average of the spike count of the neuronal unit to 

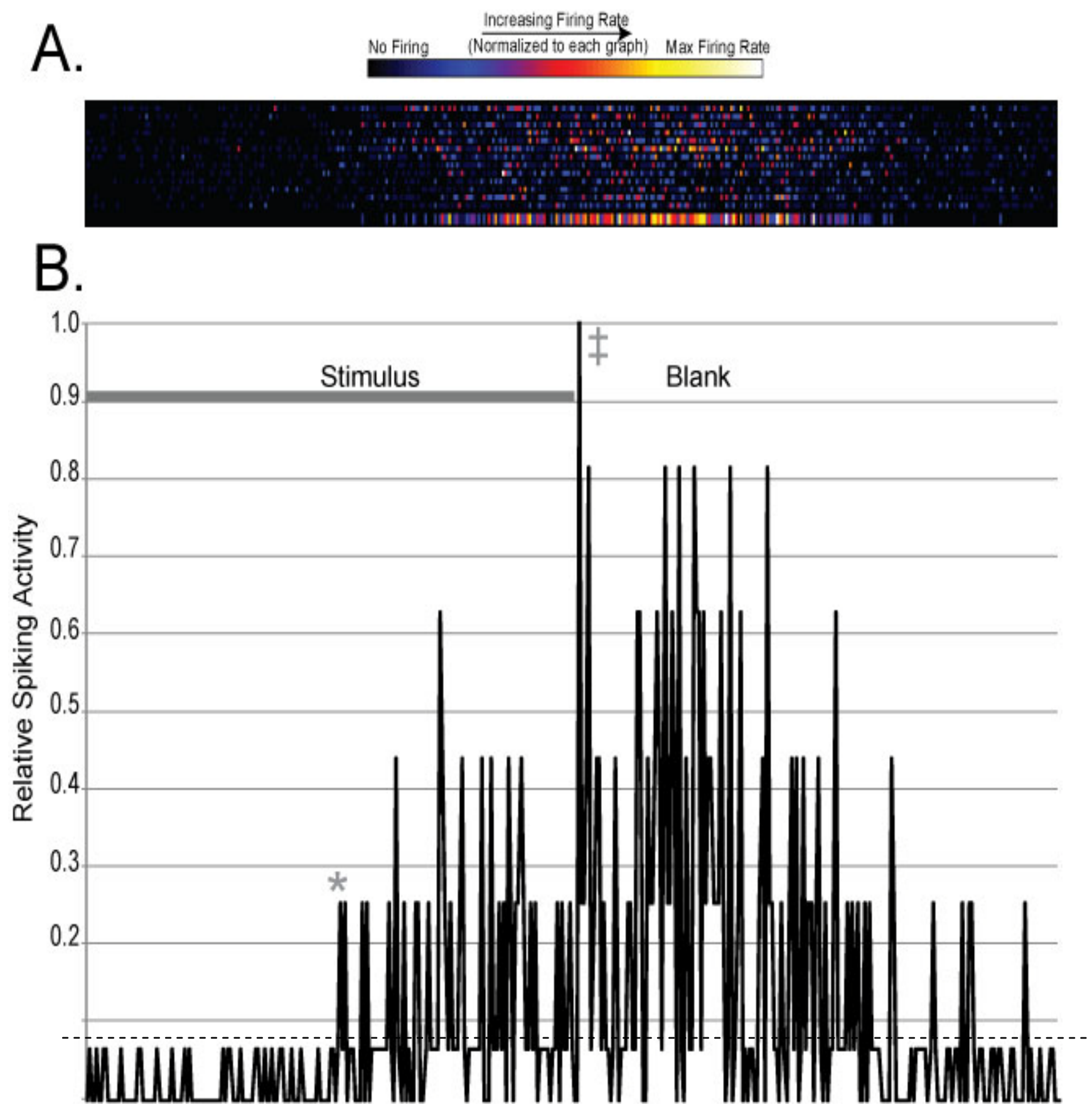

Figure 4-2. Exemplar cell for preference determination and temporal properties characterization. For each cell, the spiking activity was examined in a 2D histogram $(A)$ and as the averaged temporal spiking response (B) for each cell to calculate preference and latency. From this particular cell, the spiking has been normalized to graphically indicate intensity of spiking in each $1 \mathrm{msec}$ bin of the 2D histogram for all potential color/white point presentations of the stimulus (A). After preference determination, we examine the relative spiking activity to determine baseline activity, latency, and maximum firing time point $(B)$. The baseline noise, indicated by the dashed line, was calculated as $10 \%$ of the total spiking activity (not relative spiking activity). Spiking activity was determined to be significant if it was $>2$ std above the baseline noise. The * indicates our calculated latency point: the first significant spiking activity after the onset of the stimulus. A second temporal characteristic we examined, the global maximum or max firing latency, is indicated with the $\neq$. 
the particular stimulus and the counts of the two surrounding stimuli) were examined. A total spike count must be $>10 \%$ of the mean spike count to be considered significant. Calculations between the activity of a candidate stimulus and all other stimuli were examined, with $>10 \%$ again being the cutoff. The "latency" was defined similarly; the first time point or bin displaying significant spiking activity after the presentation of the stimulus. Based upon previously reported data (Munk et al., 1995; Schmolesky et al., 1998), observed latencies must be within 50-200 msec. These calculations provided one facet of the cellular spiking response to color and orientation.

\subsubsection{Temporal Response Characteristics:}

One of the temporal response characteristics we examined was point of maximum firing rate, or global maximum. The calculated point of maximum firing rate is defined as the bin in which the spiking rate is at the maximum point of activity of the entire response, above baseline, and $>10 \%$ above the mean spike count (Figure 4-2B). To investigate changes that occurred to a cell's spiking response with differing stimuli, it was necessary to average the results of all cells in the stimulus groups. The number of significant spikes were grouped into $1 \mathrm{msec}$ bins across the 400 msec stimulus presentation window. Plotting of the arithmetic mean and standard deviation of each bin allowed further investigation to the effects of color on a cell's spiking activity. Significant difference was calculated using a t-Test of Means, indicating the bins that differed between the two stimulus groups. These points of difference suggest time periods in a cell's activity that were affected by the addition of color to the orientation bar. Further analysis for potential differences was examined by adding unmatched cells to the averages (cells that did not have corresponding achromatic, preferred-orientation or preferred-color, preferredorientation recordings). The addition of these cells granted us a larger population of cells to examine for response changes. 


\subsection{Results}

Using optical imaging maps to guide single unit recordings, we recorded and examined the temporal responses of V2 cells in monkeys (Figure 4-1). Functional images were collected to horizontal/vertical oriented and achromatic/chromatic stimulus conditions. Using (horizontal vertical) and (color - luminance) frame difference maps, in conjunction with a surface vessel map, we identified the functional border of V1 and V2 and then targeted V2 in order to localize units for further analysis with single-unit electrophysiology (Ramsden et al., 2001). After locating an acceptably well-driven cell, the receptive field was plotted on the projection screen using an in-house computerized receptive field mapping routine. Subsequent stimulus presentations using our stimulus presentation computer program were then centered over the geometric center of the plotted RF. Receptive fields were reconfirmed during the recording procedures. We then recorded and calculated the temporal spiking responses from each cell to two stimulus conditions: the first condition (ABar) was an achromatic (white) bar of the cell's preferred width, length, and orientation while the second stimulus (CBar) was comprised of the cell's preferred width, length, orientation, and color. Thus the primary difference between the two stimulus conditions were the presence or absence of color. Figure 4-2 is an exemplar temporal profile of a cell to our CBar stimulus, illustrating preference determination and other temporal property analysis.

The examination and analysis of our data indicates that the addition of color to an orientation stimulus yielded a significantly longer averaged latency in neurons. Additionally the mean time of peak firing rate displayed a significantly delayed latency. The overall temporal response to the compound stimulus showed a more complex response: a significant reduction in the initial, transient phase but a preserved later, sustained spiking response. However, no significant correlation of latency or maximum firing times was discernible to orientation or color preference, total spiking activity, tuning width, or between the two times (latency vs. max firing time).

The frequency distributions of latencies for all of sampled cells (cells with and without corresponding ABar/CBar recordings) are presented in Figure 4-3A while the range of maximum spiking time points is shown in Figure 4-3B. These ranges of responses indicate a mean response latency of $97.43 \pm 26.64$ msec (mean \pm standard deviation) $\mathrm{N}=42$ for an achromatic stimulus and a mean latency of $92.94 \pm 33.08 \mathrm{msec} \mathrm{N}=34$ for a preferred chromatic stimulus, indicating 
no significant difference between the two times (t-Test: Two-Sample Assuming Unequal Variances, $\mathrm{p}=0.524)$. The maximum firing times also showed no significant difference between chromatic and achromatic stimulation; $212.85 \pm 99.89 \mathrm{msec}$ and $169.76 \pm 88.37 \mathrm{msec}$, respectively (t-Test: Two-Sample Assuming Unequal Variances, p = 0.053).
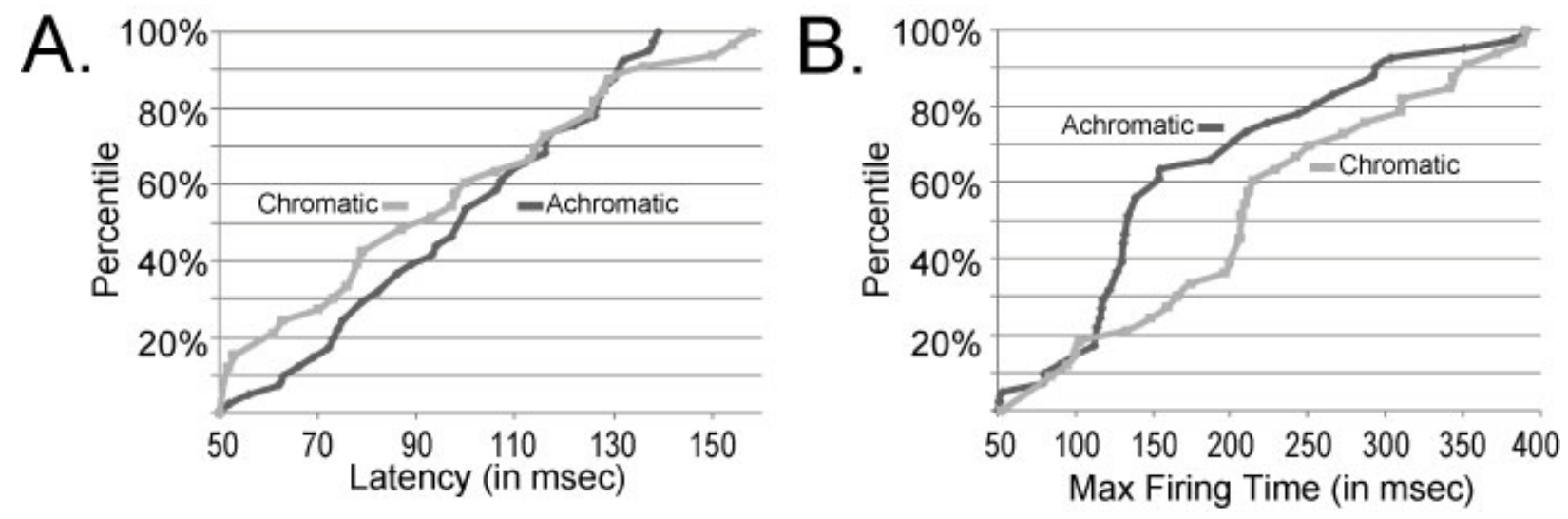

Figure 4-3. Distribution of latencies and maximum firing times. (A) Cumulative distribution of latencies to an achromatic, preferred-orientation $\mathrm{N}=42$ and preferred-color, preferred-orientation stimulus $\mathrm{N}=34$. (B) Cumulative distribution of times of maximum firing rate to an achromatic, preferred-orientation $\mathrm{N}=42$ and preferred-color, preferred-orientation stimulus $\mathrm{N}=34$. Percentile is plotted as a function of time post stimulus presentation. The addition of color lengthens the latency and adds to the time until maximum spiking rate.

When comparing the temporal spiking activities of a subset of cells with suitable recordings to both achromatic and chromatic orientation bars, a significant difference was observed; cells displayed an average latency of $86.70 \pm 18.02 \mathrm{msec} N=23$ when the achromatic stimulus was presented and this average increased to $102.70 \pm 27.05 \mathrm{msec} \mathrm{N}=23$ when the preferred color was utilized. Using a t-Test Paired Two Sample for Means, the p-value for a twotailed t- test between these latencies was calculated as $p=0.005$, with the statistical power at $76.3 \%$. These results indicate a significant difference between the average latencies of a single cell to an achromatic, orientation-preferred stimuli and a color-preferred, orientation-preferred stimuli, suggesting that even when the preferred-color is combined with a preferred-orientation stimulus, the addition of low level, information to a previously achromatic stimulus can increase the time until significant spiking activity occurs.

Interestingly, not all cells responded in a similar fashion. While most displayed a resulting increase in latency, eight cells displayed a decrease in latency when the color modality was added to a preferred orientation bar stimulus. Figure 4-4. plots the changes in latency for each of the matched cells recorded: panel $4 A$ displays the recorded achromatic and chromatic 
latency for each of the cells. The unity bar indicates no change in latency with the addition of color; most points lie above this line, indicating an increase in latency with the addition of color. Each of the cells' two latencies are further illustrated in panel $4 B$, further demonstrating the increase in latency with the addition of color.
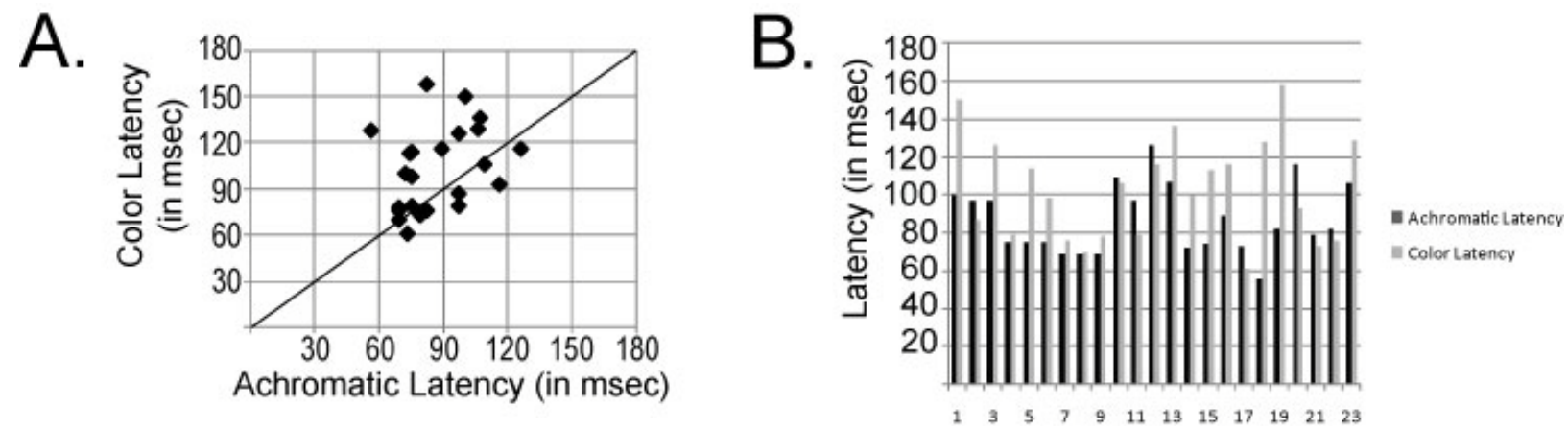

Figure 4-4. Latency modulation due to addition of color to orientation bar. Latencies were calculated using a bar visual stimulus configured to a cell's preferred width, length, and orientation at a luminance of $37 \mathrm{~cd} / \mathrm{m}^{2} . \mathrm{N}=23$. (A) Modulations in latency with addition of a cell's preferred color to bar stimulus. The scatter plot demarcates each cell's achromatic bar latency versus the same cell's preferred color bar latency. The solid black lines plots a 1:1 ratio, indicating no difference between the two. The majority of points fall above the unity line, showing an increase in latency with the addition of color to the stimulus. (B) Each pair of recorded latencies is indicated in histogram form, displaying the achromatic and color bar latency for the examined cells. While the majority of cells show an increase in latency, responses are highly heterogeneous. Though some latencies did not significantly change and some shortened, a t-Test: Paired Two Sample for Means indicates that the addition of the color significantly lengthens the latency of a cell in response to the combined stimulus $(p=0.005)$.

The time point of maximum spiking was also affected by the presence of color in the oriented stimuli. When presented with an achromatic stimulus, maximum spiking occurred at $185.53 \pm 80.54$ msec $\mathrm{N}=23$ from stimulus onset as opposed to $237.22 \pm 84.88 \mathrm{msec} \mathrm{N}=23$ when the preferred color was a combined part of the stimulus. A t-Test Paired Two Sample for Means indicates this change in spiking time is significant ( $p \geq 0.01$ with a statistical power of 68.2\%) and overall an increase in the length of time until maximum spiking occurs when color is added to an orientation bar. Again, not all cells displayed a similar response to the addition of the color modality: seven cells displayed a decrease in time to maximum spiking activity. Furthermore, these particular seven cells differed from the cells that displayed a decrease in latency with color. In other words, cells that showed a decrease in latency were not necessarily the same cells that displayed a shortening in time to maximum spiking activity (Figure 4-5). Similar to the changes in latency, most points are above the unity line and indicate a lengthening of time until the maximum firing point (Figure 4-5A). 
We investigated whether the preferential properties or quantitative spiking activity of the cells had a relationship with our calculated temporal response characteristics. An analysis of the correlation of a cell's orientation preference to latency ( $r=-0.0431$, Figure 4-6A) and max firing time ( $r=0.0890$, Figure 4-6B) suggest that there is no discernible correlation. The orientation preference of the cells did not correlate with our metrics of temporal properties. A comparison of obtained latency compared with preferred color ( $r=-0.0755$, Figure 4-6C) and max firing time ( $\mathrm{r}$ $=0.1616$, Figure 4-6D) yielded similar results and showed no strong correlation between color preference and latency or maximum firing time.
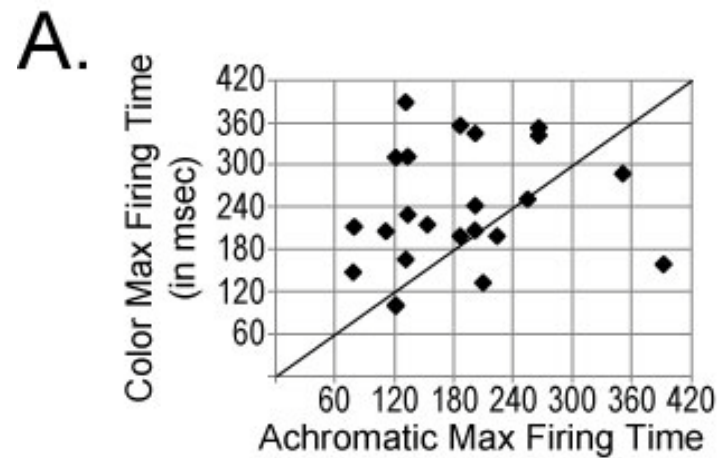

(in $\mathrm{msec}$ )

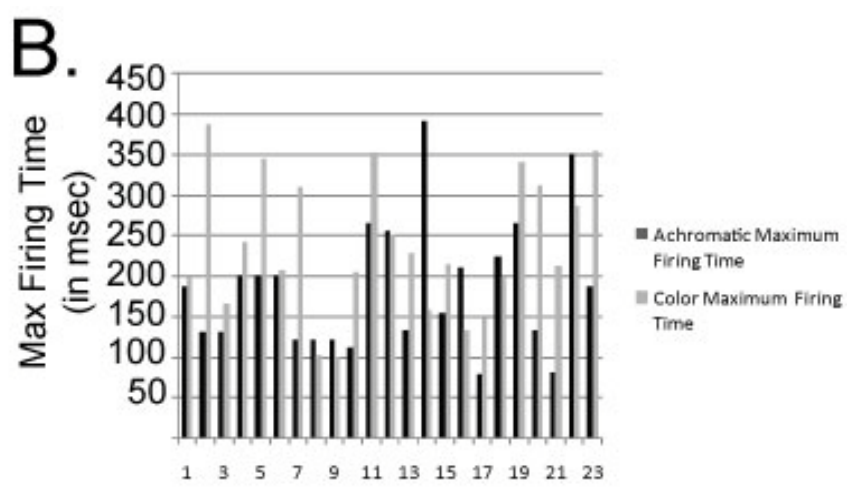

Figure 4-5. Addition of color to an orientation bar lengthens time to peak firing rate. As with the other temporal properties, maximum firing rate time was calculated using a bar visual stimulus configured to a cell's preferred width, length, and orientation at a luminance of $37 \mathrm{~cd} / \mathrm{m}^{2} . \mathrm{N}=23$. (A) The comparison of the achromatic and chromatic maximum firing times. The solid black line represents the unity where both firing times are identical and there is no change with the addition of color to the stimulus. Most points fall above the line, showing an increase in maximum firing times. (B) For each of the 23 cells, the achromatic and chromatic peak firing times. Most cells show a lengthened time until maximum spiking activity though some display shortened time periods. A t-Test Paired Two Sample for Means indicates that the addition of color significantly lengthens the maximum firing response of a cell in as a result of the preferentially colored stimulus $(p=.01)$.

The total spike count of a cell showed some correlation to colored orientation bars. A comparison of the total number of spikes per cell per achromatic stimulation suggested no relationship between spike count and achromatic max firing time ( $\mathrm{r}=0.0102$, Figure 4-7A). However, the examination of a cell's maximum firing time as a function of total spike count suggests a moderate correlation ( $\mathrm{r}=-0.3362$, Figure 4-7B), indicating an inverse relationship between spiking activity and global maximum. In other words, as the number of significant spikes during chromatic stimulation increases, the maximum firing time decreases, or lessens.

We observed an intriguing inverse relationship between a cell's change in latency and change in maximum firing time due to the addition of color to an oriented bar $(r=-0.2517$, 
Figure 4-8). This moderate correlation shows that as the maximum firing rate time increases as a result of the addition of color to the stimulus bar, a decrease in latency was more likely. Our data suggest that a cell either displayed an increase in latency with the addition of the colored stimuli or a lengthening of time until the maximum firing rate, but typically did not display both.
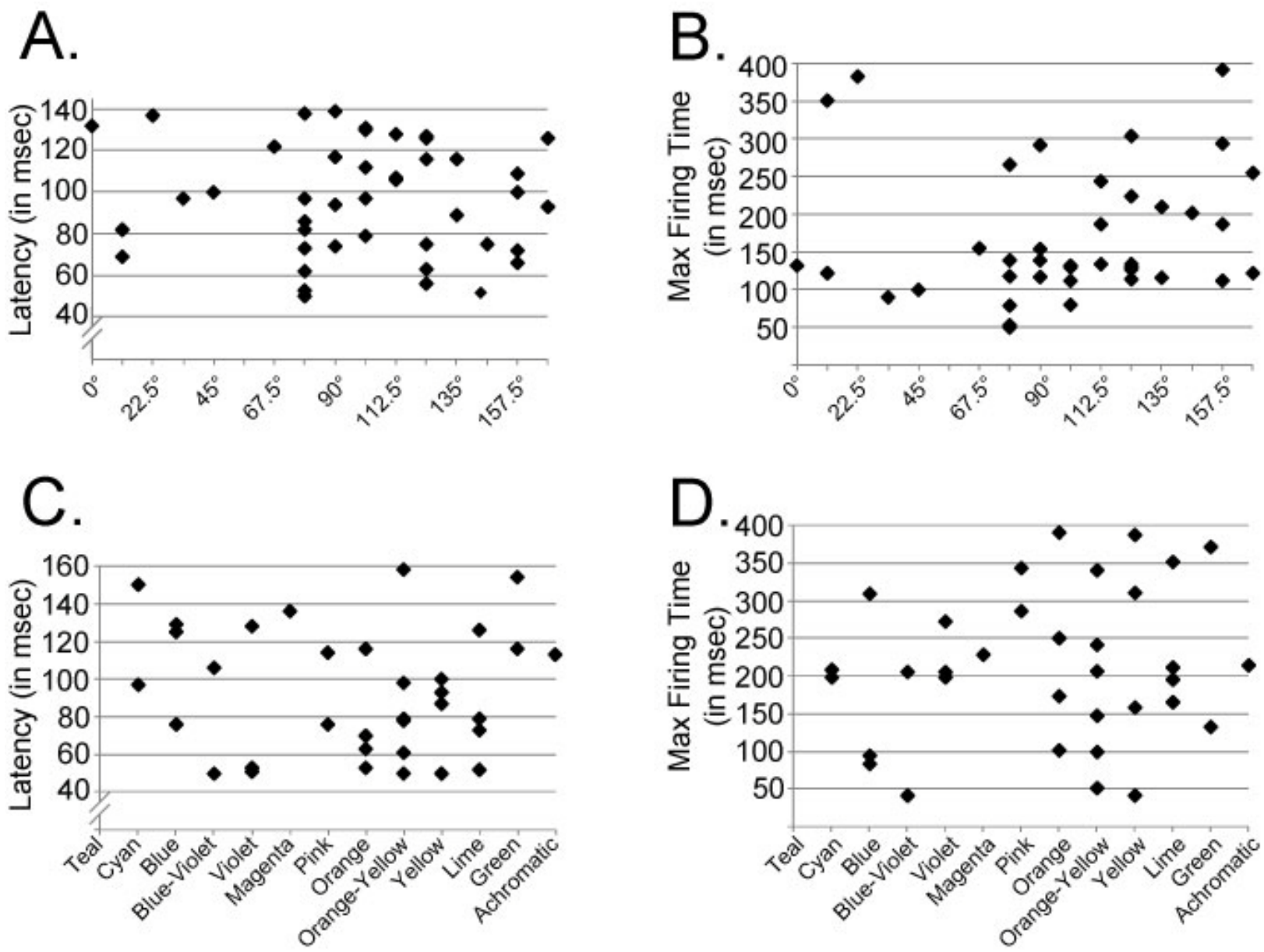

Figure 4-6. Orientation and color preference do not affect latency or maximum firing times. Examination of the cells orientation and color preferences on temporal properties. A cell's orientation angle preference has no discernable effect on the recorded latency $(A)$ or the maximum firing time $(B)$. The calculated correlations for each of the analyses of orientation were -0.043058201 and 0.088956421 , respectively. The cell's preferred color also has no effect on the observed latency $(r=-0.07550902)(C)$ and a very little effect on the max firing time point $(r=0.161600696)(D)$.We observed an intriguing inverse relationship between a cell's change in latency and change in maximum firing time due to the addition of color to an oriented bar $(r=-0.2517$, Figure $4-8)$. This moderate correlation shows that as the maximum firing rate time increases as a result of a colored bar, a decrease in latency was more likely. Our data suggest that a cell either displayed an increase in latency with the addition of the colored stimuli or a lengthening of time until the maximum firing rate, but typically did not display both. $\mathrm{N}=23$ 
The temporal response or shape of the spiking activity was also affected by the addition of color. The spiking activity was binned in 1 msec intervals and averaged within each stimulus group. Figure 4-9A and $9 B$ show the average response of all cells to an achromatic, preferredorientation bar and a preferred-color, preferred-orientation bar, respectively. A bin-by-bin statistical analysis across all groups determined an individual bin's average spiking activity and standard deviation. The averaged responses of the achromatic and colored stimulus groups are combined in panels $9 C$ and 9D. A t-Test Paired Two Sample for Means was conducted at each bin to determine the points in time which significant differences in spiking response occurred. A significant difference between the achromatic and colored oriented response can be observed in the initial onset phase of the temporal response. The time period between $100 \mathrm{msec}$ and 130 msec display a significant alteration in spiking activity, with the addition of color yielding a decrease in spikes-per-bin. This decrease is transient, however, and the color response returns to a level of activity indistinguishable from an achromatic bar beyond this time window. Panel 9C shows a compilation of the same matched cells previously investigated. When these averaged profiles are bolstered with additional examples of cells' temporal spiking activity, the discrepancy between the color and achromatic remains significant (Figure 4-9D).
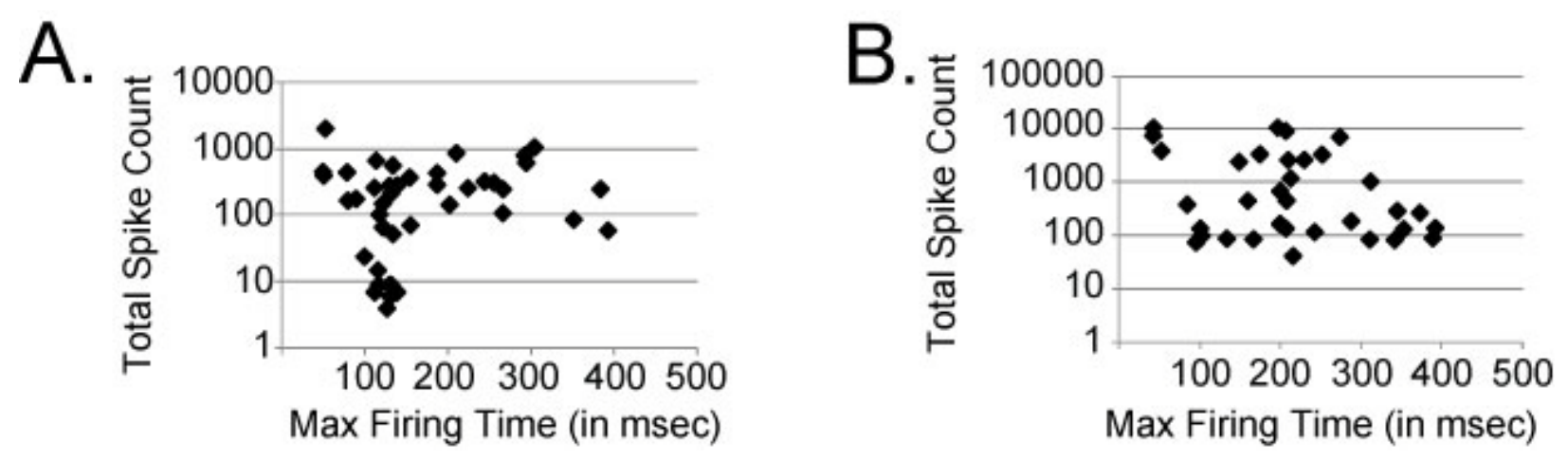

Figure 4-7. Chromatic bar maximum firing times and maximum spiking activity show some correlation. (A) Analyses of orientation maximum firing times and maximum spiking activity suggest no correlation between the two metrics $(r=0.01023764)$. (B) However, examination of a cell's maximum firing time to the number of significant spikes suggests a moderate correlation $(\mathrm{r}=$ 0.336153747). This correlation indicates as the number of significant spikes are observed from a cell presented with a colored bar increases, the maximum firing time decreases (i.e. the global maximum is less). $\mathrm{N}=23$ 


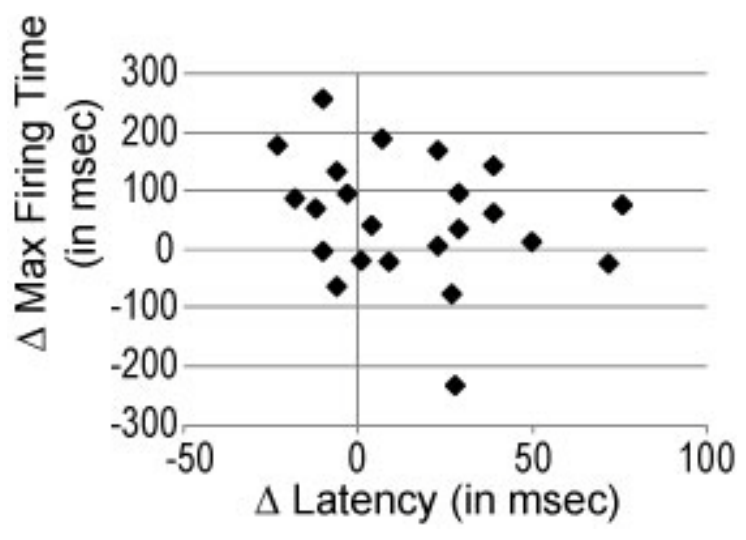

Figure 4-8. Change in latency with addition of color to an orientation bar is correlated to the change in maximum firing time. With the addition of color to the stimulus, a moderate correlation is observed between latency and maximum firing times $(r=-0.251656169)$. If the time of maximum firing rate time displayed an increase, a decrease in latency was more likely observed and vice versa, suggesting that a colored stimulus either increases latency or increases the time maximum firing rate time inversely. $\mathrm{N}=23$

\subsection{Discussion}

Our results indicated that the addition of color to a preferred achromatic orientated stimulus yielded diverse temporal response modulations in a set of anesthetized macaque V2 neurons. These findings can be summarized as follows: 1) The addition of a preferred color to the stimulus produced a significant change in mean latency overall, with nearly two-thirds of the cells displaying a significant increase in latency; 2) The addition of color to the achromatic stimulus typically brought about a significant increase in mean time to peak firing rate; and, 3) The presentation of preferred-color and preferred-orientation stimulus generated a significant reduction in the initial, transient phase but preserved the later, sustained response, when calculated using mean spiking activities.

Although our calculated results of latencies are computed from chromatic/achromatic stimulus presentations to the same cell, our reported latency values (and their relative differences) are consistent with those results previously described in different populations of color- and orientation-preferring cells (Munk et al., 1995; Schmolesky et al., 1998). We show a significant increase by an average of $16 \mathrm{msec}$ in a single cell's latency when the preferred color was added to a preferred orientation bar. Understanding latencies of neurons in response to stimuli are also thought to be important factors in attention models (Oram et al., 2002) and rapid behavioral responses (VanRullen et al., 2005). While it has been reported that "higher" visual cortical levels display longer latencies then those of earlier cortices (Raiguel et al., 1989; Maunsell and Gibson, 1992), previous reports of recordings from V2 cells have-not comprehensively examined how the same cell's response can be differentially modified as a reaction to a changing stimulus; our results suggest that temporal properties are mutable and stimulus-dependent. A cell possessing two different latencies for two different facets of visual 
stimulation might suggest separate pathways of innervations at the level of a single neuron (e.g. a neuron within a pale stripe, or a color-selective neuron).
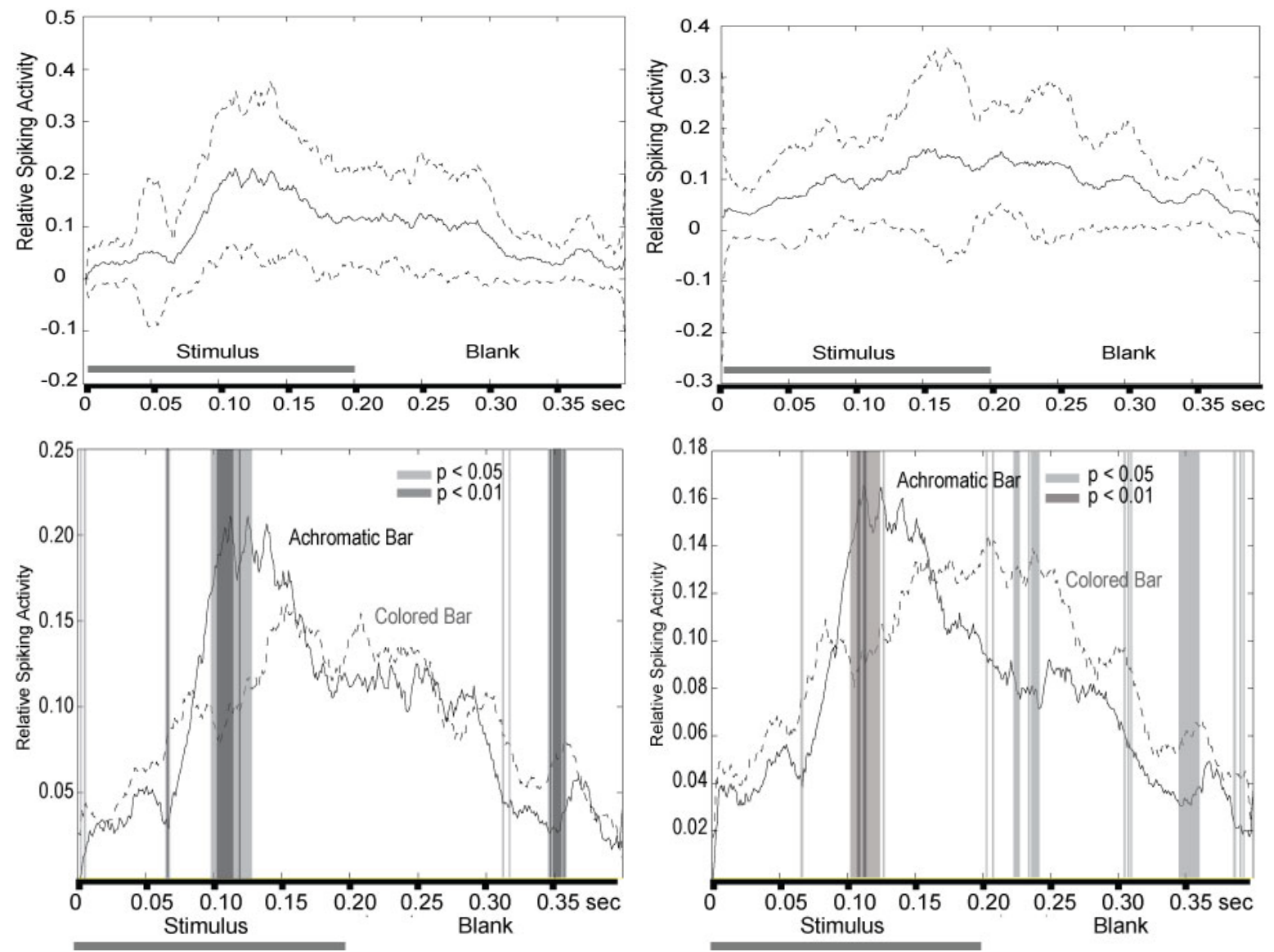

Figure 4-9. Averaged temporal response profile of cells to preferred-color, preferred orientation stimuli display reduced spiking response during the initial phase of activity compared to preferred-color, preferred orientation stimuli. (A) and (B) both show averaged response and associated standard deviation of the 23 cells over the course of a stimulus presentation trial. Solid black line traces average spiking response; two dashed lines indicate standard deviation lines, as calculated by a $1 \mathrm{msec}$ bin-by-bin analysis of the spiking activity. (A) Averaged response of all cells with achromatic, preferred-orientation stimulus. (B) Averaged spiking activity to preferred-color, preferred-orientation stimulus. $(C)$ Overlay of the two averaged spiking responses, where solid line traces average spiking activity of cells to an achromatic stimulus; dashed line shows the average response of the same cells to a preferred-color bar. Differences between the two averages were also calculated by examining each averaged bin with a t-Test for Paired Means. $N=23$ Significant differences are marked on the graph: deviations of $p<0.05$ are highlighted in light grey while deviations of $p<0.01$ are highlighted in dark grey. (D) Compilation and average of the same 23 cells plus additional unmatched cells (no corresponding achromatic or preferred-color trace). In total, there are 42 recordings of cells to an achromatic, preferred-orientation stimulus and 34 preferred-color, preferred-orientation stimulus recordings. The achromatic response is reported by the solid black line, the colored-bar response by the dashed line. Significant differences of $p<0.05$ and $p<0.01$ are highlighted in light grey and dark grey, respectively. Both $C$ and $D$ denote the significantly reduced spiking response during the initial phase of activity $(100-130 \mathrm{msec})$ with a colored-bar stimulus; latter phase of spiking activity shows no significant difference. 
We further investigated other temporal response measures in our cell population. Maximum spiking, or the global maximum, was one such type of temporal response we examined. The point at which maximum firing occurred increased by an average of approximately $52 \mathrm{msec}$ when a cell was presented with a preferred-color, preferred-orientation stimulus, when compared with achromatic preferred orientation stimulus. We also observed a significant decrease in the amount of initial activity. The addition of chromatic information yielded an averaged “dampening” of activity during the $100-130 \mathrm{msec}$ time period of the temporal response. Another possibility is that local circuitry could play a role in this inhibition resulting in this modification since the decreased response was mostly transient while the remainder of the cell's response remained (Douglas et al., 1995).

These obtained results are surprising, and are not simply attributable either to changes in the relative activation of the different projections associated with the lateral geniculate (e.g., magnocellular vs. parvocellular) or to changes in the relative activations of the supposed bipartite projection pathways from V1 onto V2. A general reduction in the initial 'fast' response components might be expected, for example, if the luminance component of the stimulus (activating the relatively rapid magnocellular pathways) was reduced from one stimulus to the next. However, the luminance of the stimuli were always held constant. Only the chromatic component were manipulated and these colored stimulus parameters are more commonly associated with the relatively slower parvocellular (or koniocellular) rather than magnocellular activations. A third pathway, the koniocellular pathway, sends projections from S-cones that arrive relatively late into V1 (Cottaris and De Valois, 1998; Casagrande, 1999; Hendry and Reid, 2000). The supposed bipartite projections from V1 to V2 (suggesting relatively distinct pathways of color and orientation into the secondary visual cortex) also do not simply explain our obtained differences in temporal response(Livingstone and Hubel, 1984; Hubel and Livingstone, 1987; Sincich and Horton, 2002). Mixed magnocellular and parvocellular cellular inputs have been suggested to underlie the cortical circuits in V1 that process both color (blob) and orientation (inter-blob) (Lachica et al., 1992). Thus, a manipulation of only chromatic information (not luminance) might be expected to influence either pathway from V1 to V2 (blob to thin, interblob to pale/thick), and lead to changes only in later (parvocellular) not earlier (magnocellular) temporal components as found in our data analysis (Maunsell et al., 1999). 
A compound stimulus of color and orientation might be expected to evoke a temporal response similar to its fastest component - orientation (Johnson et al., 2004). Since we observed an increase in response time, this would suggest that the slower color information dominates while the faster orientation component is relatively suppressed. Magnocellular channels have been suggested to inhibit parvocellular channels during the attentional processing of transient visual information (Breitmeyer and Ganz, 1976), but the converse -- inhibition of magnocellular channel by parvocellular channel --- is not well described. The reduction of initial temporal components on the introduction of color to the presented achromatic stimulus conditions would be consistent with a possible suppression of magnocellular channel by parvocellular (and or koniocellular) channels.

An alternative possibility is that these interactions are occurring locally in Area V2. Recent research has demonstrated some V2 cells prefer a more complex compound stimuli comprising multiple orientated bars, or the conjunction of spatially separate color and orientation compound stimuli with non-linear results (Ito and Komatsu, 2004; Hinkle and Connor, 2005; Anzai et al., 2007), and that this could arise via a complicated interaction involving multiple cells in V2. A non-linear summation occurs when the net response of a cell to multiple stimuli is not equal to the sum of the responses to individual stimuli. The changes in temporal responses observed in our data may also be a consequence of complex interactions involving circuitry with V2 that involves both excitatory and inhibitory processes. Large scale organizations (domains) with functional preferences specifically associated with the stimulus preferences manipulated in our stimuli (color and orientation), and could play a dominant intra-areally mediated role in the combination of (or balance of) responses to individual stimulus components.

Our experimental results are not in direct agreement with (but are not totally inconsistent with) the reaction times (RT) observed in previous psychophysical studies. While their perceptual results would suggest that they expect RT for all our stimulus conditions (chromatic and achromatic) should not be significantly different (Holmes, 1926; Lit et al., 1971; Mollon and Krauskopf, 1973; Nissen and Pokorny, 1977), we nevertheless observed a significant increase in latency with the addition of color to the oriented bar. However, this result is predicted by the perceptual studies with a background of equal luminance, which reported that color stimuli have a longer RT time than achromatic stimuli in equiluminant conditions. (In contrast, we used stimuli with a measured luminance of $37 \mathrm{~cd} / \mathrm{m}^{2}$ on a $6 \mathrm{~cd} / \mathrm{m}^{2}$ darkened screen). Interestingly, our 
temporal results did not show any readily discernible association to the spectral colors as observed in other psychophysical studies (McKeefry et al., 2003) although like all of our complex temporal data a definitive correlation is not absolute.

In summary, the intricate network of cellular connections likely needed to yield neurons within multiple modality preferences and variable temporal properties (as found in our data analysis) is difficult to describe using the segregated bipartite model of projections from V1 onto V2, or by the known patterns of interactions of magnocellular vs. parvocellular inputs. While the recorded modulation of temporal responses documented from the in vivo data might involve changes in the balance of activation of the various input pathways contributing to the inputs innervating a single neuron within $\mathrm{V} 2$, an intra-areal mechanism seems to be a likely candidate. The likelihood of strong local interactions in V2 are well documented and there is close proximity of functional organizations of neurons (domains) that each prefer the color or orientations directly manipulated in the experiments (Levitt et al., 1994b; Kiper et al., 1997; Shipp and Zeki, 2002). 


\subsection{References}

Anzai A, Peng X, Van Essen DC (2007) Neurons in monkey visual area V2 encode combinations of orientations. Nat Neurosci 10:1313-1321.

Breitmeyer BG, Ganz L (1976) Implications of sustained and transient channels for theories of visual pattern masking, saccadic suppression, and information processing. Psychol Rev 83:1-36.

Casagrande VA (1999) The mystery of the visual system K pathway. J Physiol 517 (Pt 3):630.

Chen LM, Heider B, Williams GV, Healy FL, Ramsden BM, Roe AW (2002) A chamber and artificial dura method for long-term optical imaging in the monkey. J Neurosci Methods 113:41-49.

Cottaris NP, De Valois RL (1998) Temporal dynamics of chromatic tuning in macaque primary visual cortex. Nature 395:896-900.

Derrington AM, Krauskopf J, Lennie P (1984) Chromatic mechanisms in lateral geniculate nucleus of macaque. J Physiol 357:241-265.

Douglas RJ, Koch C, Mahowald M, Martin KA, Suarez HH (1995) Recurrent excitation in neocortical circuits. Science 269:981-985.

Fries P, Neuenschwander S, Engel AK, Goebel R, Singer W (2001) Rapid feature selective neuronal synchronization through correlated latency shifting. Nat Neurosci 4:194-200.

Frostig RD, Lieke EE, Ts'o DY, Grinvald A (1990) Cortical functional architecture and local coupling between neuronal activity and the microcirculation revealed by in vivo highresolution optical imaging of intrinsic signals. Proc Natl Acad Sci U S A 87:6082-6086.

Gegenfurtner KR, Kiper DC (2003) Color vision. Annu Rev Neurosci 26:181-206.

Gilbert CD, Wiesel TN (1990) The influence of contextual stimuli on the orientation selectivity of cells in primary visual cortex of the cat. Vision Res 30:1689-1701.

Grinvald A, Lieke E, Frostig RD, Gilbert CD, Wiesel TN (1986) Functional architecture of cortex revealed by optical imaging of intrinsic signals. Nature 324:361-364.

Hendry SH, Reid RC (2000) The koniocellular pathway in primate vision. Annu Rev Neurosci 23:127-153.

Hinkle DA, Connor CE (2005) Quantitative characterization of disparity tuning in ventral pathway area V4. J Neurophysiol 94:2726-2737.

Holmes JL (1926) Reaction time to photometrically equal chromatic stimuli. American Journal of Psychology 37:414-417. 
Horton JC (1984) Cytochrome oxidase patches: a new cytoarchitectonic feature of monkey visual cortex. Philos Trans R Soc Lond B Biol Sci 304:199-253.

Hubel DH, Livingstone MS (1987) Segregation of form, color, and stereopsis in primate area 18. J Neurosci 7:3378-3415.

Ito M, Komatsu H (2004) Representation of angles embedded within contour stimuli in area V2 of macaque monkeys. J Neurosci 24:3313-3324.

Johnson EN, Hawken MJ, Shapley R (2004) Cone inputs in macaque primary visual cortex. J Neurophysiol 91:2501-2514.

Kiper DC, Fenstemaker SB, Gegenfurtner KR (1997) Chromatic properties of neurons in macaque area V2. Vis Neurosci 14:1061-1072.

Knoblauch A, Palm G (2002) Scene segmentation by spike synchronization in reciprocally connected visual areas. I. Local effects of cortical feedback. Biol Cybern 87:151-167.

Lachica EA, Beck PD, Casagrande VA (1992) Parallel pathways in macaque monkey striate cortex: anatomically defined columns in layer III. Proc Natl Acad Sci U S A 89:35663570 .

Levitt JB, Kiper DC, Movshon JA (1994a) Receptive fields and functional architecture of macaque V2. J Neurophysiol 71:2517-2542.

Levitt JB, Yoshioka T, Lund JS (1994b) Intrinsic cortical connections in macaque visual area V2: evidence for interaction between different functional streams. J Comp Neurol 342:551-570.

Lit A, Young RH, Shaffer M (1971) Simple reaction time as a function of luminance for various wavelengths. Perception \& Psychophysics 10:397-399.

Livingstone MS, Hubel DH (1983) Specificity of cortico-cortical connections in monkey visual system. Nature 304:531-534.

Livingstone MS, Hubel DH (1984) Anatomy and physiology of a color system in the primate visual cortex. J Neurosci 4:309-356.

Malach R, Amir Y, Harel M, Grinvald A (1993) Relationship between intrinsic connections and functional architecture revealed by optical imaging and in vivo targeted biocytin injections in primate striate cortex. Proc Natl Acad Sci U S A 90:10469-10473.

Maunsell JH, Gibson JR (1992) Visual response latencies in striate cortex of the macaque monkey. J Neurophysiol 68:1332-1344. 
Maunsell JH, Ghose GM, Assad JA, McAdams CJ, Boudreau CE, Noerager BD (1999) Visual response latencies of magnocellular and parvocellular LGN neurons in macaque monkeys. Vis Neurosci 16:1-14.

McKeefry DJ, Parry NR, Murray IJ (2003) Simple reaction times in color space: the influence of chromaticity, contrast, and cone opponency. Invest Ophthalmol Vis Sci 44:2267-2276.

Mollon JD, Krauskopf J (1973) Reaction time as a measure of the temporal response properties of individual colour mechanisms. Vision Res 13:27-40.

Munk MH, Nowak LG, Girard P, Chounlamountri N, Bullier J (1995) Visual latencies in cytochrome oxidase bands of macaque area V2. Proc Natl Acad Sci U S A 92:988-992.

Nissen MJ, Pokorny J (1977) Wavelength effects on simple reaction time. Perception \& Psychophysics 22:457-462.

Olavarria JF, Van Essen DC (1997) The global pattern of cytochrome oxidase stripes in visual area V2 of the macaque monkey. Cereb Cortex 7:395-404.

Oram MW, Xiao D, Dritschel B, Payne KR (2002) The temporal resolution of neural codes: does response latency have a unique role? Philos Trans R Soc Lond B Biol Sci 357:987-1001.

Priebe NJ, Lisberger SG, Movshon JA (2006) Tuning for spatiotemporal frequency and speed in directionally selective neurons of macaque striate cortex. J Neurosci 26:2941-2950.

Raiguel SE, Lagae L, Gulyas B, Orban GA (1989) Response latencies of visual cells in macaque areas V1, V2 and V5. Brain Res 493:155-159.

Ramsden BM, Hung CP, Roe AW (2001) Real and illusory contour processing in area V1 of the primate: a cortical balancing act. Cereb Cortex 11:648-665.

Roe AW, Ts'o DY (1995) Visual topography in primate V2: multiple representation across functional stripes. J Neurosci 15:3689-3715.

Schmolesky MT, Wang Y, Hanes DP, Thompson KG, Leutgeb S, Schall JD, Leventhal AG (1998) Signal timing across the macaque visual system. J Neurophysiol 79:3272-3278.

Shipp S, Zeki S (2002) The functional organization of area V2, I: specialization across stripes and layers. Vis Neurosci 19:187-210.

Sincich LC, Horton JC (2002) Divided by cytochrome oxidase: a map of the projections from V1 to V2 in macaques. Science 295:1734-1737.

Sincich LC, Horton JC (2003) Independent projection streams from macaque striate cortex to the second visual area and middle temporal area. J Neurosci 23:5684-5692. 
Smith MA, Bair W, Movshon JA (2006) Dynamics of suppression in macaque primary visual cortex. J Neurosci 26:4826-4834.

Sporns O, Tononi G, Edelman GM (2000) Connectivity and complexity: the relationship between neuroanatomy and brain dynamics. Neural Netw 13:909-922.

Szucs A (1998) Applications of the spike density function in analysis of neuronal firing patterns. J Neurosci Methods 81:159-167.

Tamura H, Sato H, Katsuyama N, Hata Y, Tsumoto T (1996) Less segregated processing of visual information in V2 than in V1 of the monkey visual cortex. Eur J Neurosci 8:300309.

Tootell RB, Hamilton SL (1989) Functional anatomy of the second visual area (V2) in the macaque. J Neurosci 9:2620-2644.

Ts'o DY, Roe AW, Gilbert CD (2001) A hierarchy of the functional organization for color, form and disparity in primate visual area V2. Vision Res 41:1333-1349.

Vanduffel W, Tootell RB, Schoups AA, Orban GA (2002) The organization of orientation selectivity throughout macaque visual cortex. Cereb Cortex 12:647-662.

VanRullen R, Guyonneau R, Thorpe SJ (2005) Spike times make sense. Trends Neurosci 28:1-4.

Vnek N, Ramsden BM, Hung CP, Goldman-Rakic PS, Roe AW (1999) Optical imaging of functional domains in the cortex of the awake and behaving monkey. Proc Natl Acad Sci U S A 96:4057-4060.

Wang Y, Xiao Y, Felleman DJ (2006) V2 Thin Stripes Contain Spatially Organized Representations of Achromatic Luminance Change. Cereb Cortex.

Wong-Riley M (1979) Changes in the visual system of monocularly sutured or enucleated cats demonstrable with cytochrome oxidase histochemistry. Brain Res 171:11-28.

Xiao Y, Felleman DJ (2004) Projections from primary visual cortex to cytochrome oxidase thin stripes and interstripes of macaque visual area 2. Proc Natl Acad Sci U S A 101:71477151.

Xiao Y, Wang Y, Felleman DJ (2003) A spatially organized representation of colour in macaque cortical area V2. Nature 421:535-539.

Yoshioka T, Levitt JB, Lund JS (1994) Independence and merger of thalamocortical channels within macaque monkey primary visual cortex: anatomy of interlaminar projections. Vis Neurosci 11:467-489. 
Yoshioka T, Blasdel GG, Levitt JB, Lund JS (1996) Relation between patterns of intrinsic lateral connectivity, ocular dominance, and cytochrome oxidase-reactive regions in macaque monkey striate cortex. Cereb Cortex 6:297-310. 
CHAPTER 5: Materials, Methods and Techniques 
During the course of data collection for this dissertation, various technical and practical issues arose that altered or directed the course of experimentation and required immediate attention before progressing. These issues directly affected the collection of data and thus certain avenues of experimentation were not fully explored. Several techniques, preparations and methods that were used prior (and yet were vital) to the electrophysiology but were not fully elucidated in the previous chapters need to be further addressed. Additionally, the highly technical nature and the complex skills required to successfully conduct these experiments remain to be discussed. This chapter addresses these points as they directly relate to the investigation of cluster function in cortical areas, as well as their potential role in the generation of V2 neurons possessing mixed properties. 


\subsection{Anesthesia}

One of the most critical elements that affected data collection during the electrophysiology experiments was the anesthesia. It is a regulated requirement that all animals be appropriately anesthetized at all times during all of the recording procedures. The spiking activity to be recorded in experiments is however also sensitive to anesthetic depth and this sensitivity can vary with anesthetic agent selection. Precise maintenance and control of anesthesia is therefore paramount for required humane animal use, and the recording of useful data. Individual physiology required careful adjustments in the anesthesia dosage to produce the desired level. Achieving this level of appropriate anesthetic depth was critical for the electrophysiology experiments: too much anesthesia would potentially create a physiologic state in which cellular activity could not be recorded but too little anesthesia would also be inappropriate clinical care of the animal. To ensure an optimal level of balanced anesthesia and effective electrophysiological recording, several different approved anesthetic procedures were compared and investigated. To ensure that optimal anesthetic procedures were implemented, consultations were also made with WVU neurosurgeons and an anesthesiologist regarding the recent developments and ongoing use of anesthetic agents during pediatric and adult human neurosurgery. The Director of Laboratory Animal Resources, a trained laboratory animal care veterinarian also provided helpful expert input. This section outlines the various anesthetic agents and the paralytic utilized in the in vivo experiments in our continuing attempts to further improve our procedures. The anesthetics types discussed here are amnesiacs (agents that induce a loss of memory surrounding induction), analgesics (agents that inhibit the sensation of pain), and hypnotics (sedative agents). All anesthetic and paralytic agents were administered under approved protocols sanctioned by West Virginia University Animal Care and Use Committee and Office of Laboratory Animal Resources. Agent usage was fully documented in all procedures

\subsubsection{Ketamine}

In order to safely and comfortably transport the animal from its home room to the surgery and recording facility, an injection of ketamine was given intramuscularly (IM), typically of 10 $\mathrm{mg} / \mathrm{kg}$. Ketamine is an NMDA receptor antagonist (Harrison and Simmonds, 1985) that produces a dissociative anesthesia (Bergman, 1999). In this dissociation, the animal enters a trance-like 
state, allowing safe inspection and preparation of the animal prior to all procedures. The primary responses to ketamine after IM administration were sedation and some analgesia, with oral secretions controlled with atropine $(0.03 \mathrm{mg} / \mathrm{kg} \mathrm{im})$. This effect was fairly transient, typically lasting an hour. Beyond the transportation between the housing facility (and residual effects from transportation), ketamine was not used during the experimental procedure.

\subsubsection{Isoflurane}

During procedures, the animal subject was given isoflurane, an inhalation anesthetic that provided analgesic and muscle relaxation by binding with GABA, glutamate, and glycine receptors and inhibiting potassium channels (Ries and Puil, 1999; Lambert et al., 2005). Typically, this was the first anesthesia given to the animal when brought to the procedure table as it was fast and easy to administer inhalation isoflurane with a respiratory mask. Induction dose of 3-5\% in pure oxygen was typical for the first 5-10 minutes (while observing changes in heart rate) with a maintenance dosage ranging from $0.2-2 \%$ (depending on the administration of other anesthetics). After induction, orotracheal intubation delivered all inhalation anesthetics. In certain instances, isoflurane was given alone ( 2\%) however cellular activity was inversely correlated with dosage: the higher dosage of isoflurane (seemingly independent of other anesthetics) yielded a less reactive brain and increased the difficulty of locating cells to characterize with V2. While no other physiologic or cognitive pathologies were observed as a direct result of the isoflurane, recent studies have suggested a possible decline in mouse cognitive function (Bianchi et al., 2008) and apoptosis and amyloid beta aggregation in human cells (Xie et al., 2007). Isoflurane is however commonly used in conventional human anesthetic practice. In most cases we used this agent in combination with other anesthetic supplements and so the isoflurane dosages used were relatively low.

\subsubsection{Nitrous Oxide}

Nitrous oxide $\left(\mathrm{N}_{2} \mathrm{O}\right)$ is an inhalation anesthetic that is often used in the study of vision in non-human primates (Kruger and Aiple, 1988; Livingstone, 1996; Nowak et al., 1999). The dissociative anesthetic and analgesic properties of $\mathrm{N}_{2} \mathrm{O}$ arise due to two processes: (1) through action on the midbrain, $\mathrm{N}_{2} \mathrm{O}$ releases endogenous opioid peptides that then inhibit GABAergic neurons (Fujinaga and Maze, 2007) and (2) as an NMDA antagonist (Jevtovic-Todorovic et al., 
1998). Initially, nitrous oxide was administered with the isoflurane at a rate of $70 \% \mathrm{~N}_{2} \mathrm{O}$ to $30 \%$ $\mathrm{O}_{2}$. This proportion, supplemented by isoflurane levels of $0.5-1 \%$, maintained appropriate and sufficient balance of anesthesia in animal subjects. It was noted however in practice that this combination could potentially lead to the possible side-effect of swelling and this anesthetic procedure was discontinued. In the case of observation of swelling, recommended treatments included the administration of $3 \% \mathrm{NaCl} 5 \mathrm{ml} / \mathrm{kg}$ intravenously (IV), $20 \%$ mannitol $3 \mathrm{ml} / \mathrm{kg} \mathrm{IV}$, carprofen 2-4 mg/kg IM, flunixin meglumine $5 \mathrm{mg} / \mathrm{kg} \mathrm{IM}$, furosemide 1-4 mg/kg IM and/or deamethasone $0.5 \mathrm{mg} / \mathrm{kg} \mathrm{IM}$.

\subsubsection{Sufentanil Citrate}

An alternative anesthetic combination examined was inhalation isoflurane with IV administration of sufentanil citrate, an opiod analgesic. Administration of an initial loading dose of sufentanil $(5-20 \mu \mathrm{g})$ immediately followed by continuous IV infusion was necessary to provide both immediate and sustained anesthetic effects throughout a prolonged procedure. When used as part of an anesthetic regimen, a mixture of isoflurane $0.25 \%-1.5 \%$ and sufentanil $4-12 \mu \mathrm{g} / \mathrm{kg} / \mathrm{hr}$ were used. This relatively wide variation of delivery rates was common throughout the in vivo experiments, as individual (and procedure-specific) animal physiology could differ considerably, ultimately leading to numerous adjustments during the course of an experiment. Sufentanil can accumulate in body fat and other tissues with large/multiple doses or prolonged administration. Post-procedure, this cumulative effect may be reversed using the opioid receptor competitive inhibitor naloxone (0.05-0.1mg IV), allowing with a shorter post-anesthesia recovery period. The potential cumulative effects of sufentanil citrate therefore were closely monitored during procedures, and titrated accordingly, although in some cases accumulations lead to a gradual reduction in quality of spiking activity with time. Overall, the addition of sufentanil to the anesthetic regimen was successful: the animal's welfare and data collection both benefited.

\subsubsection{Propofol}

In an effort to reduce the possible cumulative effects of larger doses of sufentanil citrate, a third anesthetic was added to the isoflurane/sufentanil regimen: propofol. Propofol is a hypnotic with amnesiac, but not analgesic and without opiod properties (Euliano and 
Gravenstein, 2004), allowing combination with sufentanil (Miner and Burton, 2007). This combination would afford a lower concentration of isoflurane and sufentanil, thereby reducing the risks associated with those particular anesthetics. Furthermore, propofol's duration is very short due to its rapid distribution into peripheral tissues, with a single dose of propofol typically wearing off within minutes and a lack of the nausea often associated with opioids (Veselis et al., 1997). During induction or occasions requiring rapid acting anesthesia, 10mg of propofol IV were given. The maintenance ratio used was $8 \mu \mathrm{g} / \mathrm{kg} / \mathrm{hr}$ sufentanil to $1 \mathrm{mg} / \mathrm{kg} / \mathrm{hr}$ propofol IV. Propofol as part of the anesthetic regimen afforded more control and modification to the anesthesia administered to the animal subject. While this particular regimen of isoflurane/sufentanil/propofol was the most promising, it was initiated only in final experiments and data presented and discussed in this dissertation (Chapter 4) were not collected using this administration combination.

\subsubsection{Vercuronium Bromide}

Though not an anesthetic, a paralytic agent was necessary to prevent the eyes from drifting during the experiment. Vercuronium bromide is a non-depolarizing blocking agent. Induction dosage was $0.5-1 \mathrm{mg}$ of vercuronium bromide with a maintenance rate of 50-100 $\mu \mathrm{g} / \mathrm{kg} / \mathrm{hr}$. After termination of paralytic delivery, it took $\sim 3$ hours for the vercuronium to wash out. It is important to note that animals were only paralyzed under general anesthesia, and vital signs were continually observed and regularly recorded in case of possible responses/reactions to experimental procedures. Animals were always recovered from the action of paralytics, prior to any further recovery from general anesthesia. 


\subsection{Surgical Procedure}

The surgical procedure and placement of the cranial chamber on the macaque were both key events for a successful series of experiments and the welfare of the animal subject. Since nearly all animals would be utilized over several procedures (in some cases, certain animal subjects underwent procedures upwards of 3 months after the initial surgery), a successful surgery and recovery, optimal and secure chamber implantation, and the health of the animal were all carefully controlled for each procedure to be beneficial. Animals were artificially ventilated and also continuously monitored via EEG, EKG, respiration rate with end-tidal $\mathrm{CO}_{2}$, oximetry, blood pressure, rectal temperature and pulse-based heart-rate monitoring (Cardell MAX-1, Sharn Veterinary, Inc., Tampa, FL). All procedures were conducted under approved protocols sanctioned by West Virginia Animal Care and Use Committee and Office of Laboratory Animal Resources.

\subsubsection{Craniotomy and Durotomy}

For each initial surgery, the top of the cranium was shaved and the stubble removed with Veet (Reckitt Benckiser, Slough, UK). The scalp was then disinfected with betadyne. While under anesthesia (and no paralytic) and under sterile, aseptic surgical conditions, a rostral-caudal incision along the midline was performed from the occipital ridge to the brow ridge on the frontal bone of the skull (heart rate, blood pressure, electrocardiography (EKG), and electroencephalography (EEG) were all monitored for reactions to the incision and anesthesia was modulated accordingly). Retractors separated the incised skin flaps, and the muscles and tissues covering the skull were carefully retracted to expose a smooth cranial surface. Using a trephine, a 7/8” diameter circular craniotomy was performed 5-15 mm lateral of the midline cranial suture and 14-20 mm rostral of the occipital cranial ridge. This location places the aperture approximately over the lunate sulcus. A durotomy (surgical resection of the dura mater) using a fine scalpel and forceps exposed the cortex.

\subsubsection{Artificial Dura Hat and Chamber Implant}

Following an experiment or procedure, the cortex had to be protected. In some instances, a small piece of thermoplastic polyurethane (Tecoflex $\left.{ }^{\circledR}\right)$ ) was positioned between the dura mater and the cortex and the dura was then closed with 1-3 silk sutures. Otherwise a Tecoflex ${ }^{\circledR}$ hat was 
placed in the dura mater opening to prevent re-growth. This material is biologically inert and has been used successfully by others in similar cranial surgical and experimental procedures.

A nylon chronic chamber was placed into the newly formed aperture, allowing a single animal to be utilized over several procedures (Figure 5-1) (Chen et al., 2002). This chamber fits snugly into the craniotomy; any potential gaps were filled with a small amount of bone wax. The skull was cleaned, rinsed with $0.9 \% \mathrm{NaCl}$ solution, and treated with Copalite Varnish (Cooley \& Cooley, LTD., Houston, TX) - this permits a better bond with the dental cement. The dental cement was built up around the chamber along with three titanium cranial screws for structural support; this provided a stable, secure and sealed base. After the cement had begun to cure, the incision line was sutured with prolene to create a neat margin around the immediate chamber implant. A clean, sterile lid was then threaded into the chamber to seal the preparation area. To ensure comfort, the suture line was infiltrated with 15\% marcaine. On recovery post-surgery, the animal subject was given a required course of buprenorphine $(10-30 \mu \mathrm{g} / \mathrm{kg})$ and/or flunixin meglumine (5 mg/kg) to ensure comfort and enrofloxacin (3 mg/kg) a prophylactic antibiotic. Animals received additional regular monitoring and inspections after all surgeries. No antibiotics or antiseptic were applied within the chamber. Any signs of infection, either within or around the chamber, would require systemic antibiotics (usually enrofloxacin, or an alternative antibiotic agent if clinically appropriate) given under veterinary supervision of the Office of Laboratory Animal Research of WVU. The monkeys' behavior, food intake, and weight were closely and regularly monitored and culture swabs of the inside of the chamber were also collected frequently to detect any possible discomfort or infection.

\subsubsection{Chamber Flush}

Approximately one week following the initial surgery and on certain instances in which an experiment or full procedure could not occur, a chamber flush was performed. In these instances, the animal was retrieved from the cage and prepared for a procedure as normal. Under brief anesthesia the chamber lid was removed and the chamber interior was inspected and flushed with $\sim 50 \mathrm{cc}$ of sterile $0.9 \% \mathrm{NaCl}$ solution. Flushing provides a means to ensure thorough cleanliness of the chamber interior, and assists in the removal of any small blood clots or secretions that may adhere to the chamber interior. 

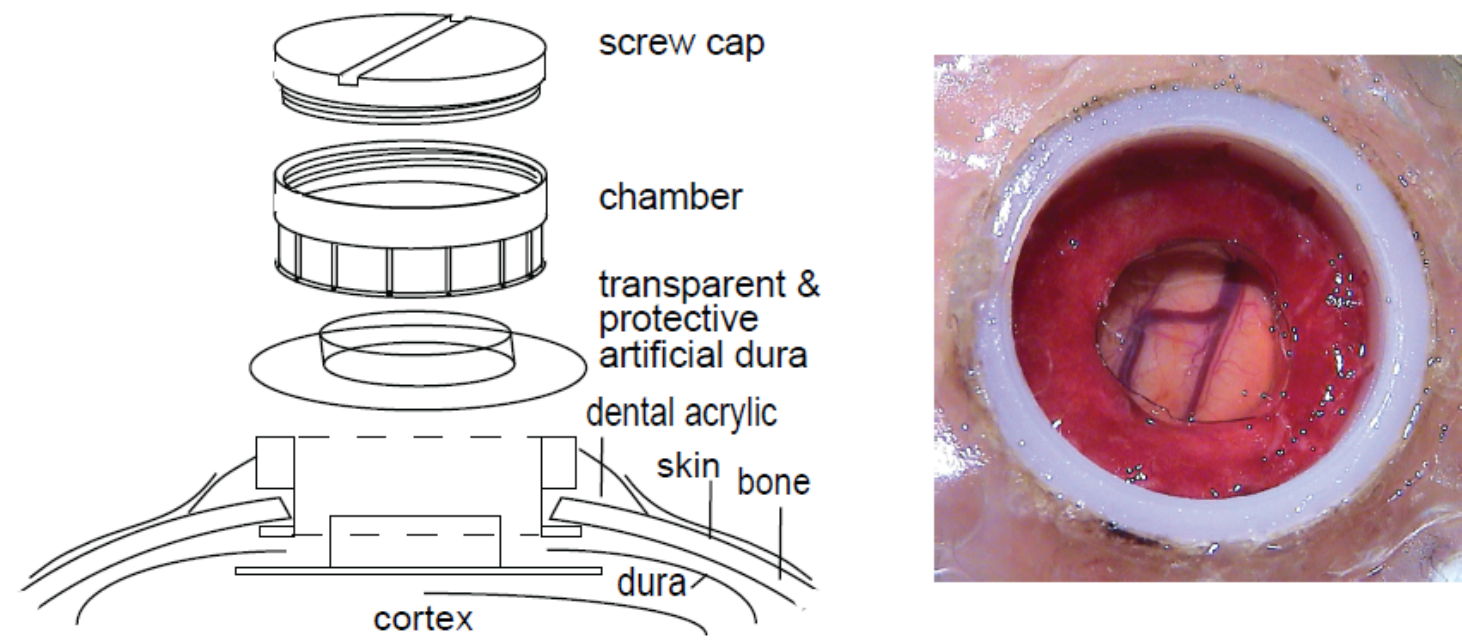

Figure 5-1. Transcranial chamber and artificial dura allow for multiple experimental procedures. After the initial craniotomy and durotomy, a re-sealable chamber was inserted and sealed with dental acrylic. To prevent the dura mater from reforming over the cortex, an artificial dura was positioned to keep the surgical area open. The screw cap allowed repeated access to the cortex of a single area over multiple 


\subsection{Intrinsic Signal Optical Imaging}

Before electrode placement occurred during the in vivo experiments described in this dissertation (Chapter 4), it was necessary to determine (cf. confirm) the location of the differing population of cells within the visual cortices (more specifically, ascertain the region of the V1/V2 border and the arrangement of the sub-population of cells specific for color- and orientation-preference in V2). Optical imaging provided a larger view of the cortex and its activity resulting from stimulation, may be the best method to determine the presence or absence of a sub-population of cells specific for color preference in V1. The functional maps created from the optical imaging provided a reference for the electrophysiology electrode placement, ensuring that the recordings were from the correct cortical area.

\subsubsection{Methodological Background}

Using visible red light, intrinsic optical imaging monitors changes of oxyhemoglobin to deoxyhemoglobin within the microcirculation of the cortex. These oxygen-state changes are purportedly related to increased neuronal activity. The optimum proposed wavelength of $\sim 605 \mathrm{~nm}$ indicates changes in oxygen state, not blood volume levels. Unfortunately this time course of metabolic change is relatively long: 200-400ms from neuron activity until oxyhemoglobin releases oxygen (resulting in a relative increase in deoxyhemoglobin), another 200-400ms before an increase in blood flow, and followed by $<1000 \mathrm{~ms}$ until the oxyhemoglobin

level returns to baseline. It is important to note that the increase in relative deoxyhemoglobin reflects cell population activity, not a single cells' response (Frostig et al., 1990). Optical imaging thus affords a larger, overall view of cortical activity but has some practical limitations in spatial and temporal resolutions.

\subsubsection{Experimental Procedure}

Before optical imaging, it was necessary to stabilize the cortex within the chamber. The brain was subject to motion not only from the animal subjects' respiration but from the pulsations of the heart rate and blood flow. To minimize and hopefully eliminate these motion artifacts, a 2-3\% agarose solution was poured into the chamber and allowed to congeal. A small window was then cut into this agarose to afford access for the optical imaging. The imaging system used to capture the intrinsic signal image was an Imager 2000 system (Optical Imaging 
Inc., Germantown, NY). The cortex was illuminated either with a pair of free-standing lights or a circular annulus light affixed to the lens of the imager. Typical imager setup consisted of an $85 \mathrm{~mm} / 50 \mathrm{~mm}$ series of attached lenses, with an F stop aperture of $2.4-4$, aligned in parallel with the cortical surface. This setup typically allowed viewing of $\sim 6-7 \mathrm{~mm}$ of visual cortex. A filter of 546 (+/- 10) nm was utilized to adjust the focus, bring the cortex and blood vessels into plane, and capture a vessel map image while intrinsic signals were imaged at $\sim 610(+/-10) \mathrm{nm}$, with the lens system focused at $400 \mu \mathrm{m}$ below the cortical surface. Signal-to-noise ratio was enhanced by averaging 40-100 trials per stimulus condition. Image acquisition was synchronized with heart rate and respiration. Electromechanical shutters (Uniblitz, Rochester, NY) in front of the eyes forced monocular viewing of the stimulus for ocular dominance. Two different full-field, sinusoidal stimuli were used: one of luminance (black and white grating) and the other consisting of red and green. The grating was randomly presented in one of four angles: $0^{\circ}, 45^{\circ}, 90^{\circ}$, and $135^{\circ}$, typically at $1 \mathrm{cpd}, 3 \mathrm{cyc} / \mathrm{sec}$. Post-image collection, difference maps were generated with single blanks and arranged over vessel maps to guide electrophysiology. 


\subsection{Single Unit Electrophysiology}

As outlined in Section 5.3, intrinsic maps allow precision targeting of single unit electrophysiology to characterize individual cells stimulus preferences and latencies. Fine, glasscoated (Micro Probe, Inc., Gaithersburg, MD) or parylene-coated (Nano Bio Sensors) tungsten electrodes were inserted into superficial layers of the V2 cortex ( 0 - $1 \mathrm{~mm}$ deep). Single unit electrophysiology was used to monitor the modulations of local neurons' spiking patterns in response to a visual stimulus (Hubel, 1957). Small increments $(5-10 \mu \mathrm{m})$ were traversed down through the layers of the cortex to isolate a single, responsive neuron. Only units that were clearly isolated, adequately and robustly "driven" with visual stimulation, and displayed significant selectivity for orientation were selected for a more intensive quantification study of orientation and/or color preference.

Initial selection of these candidate cells was based on "hand-tuning", as determined by readings from an oscilloscope (GRS-6032A, Instek, Chino, CA) and audio monitoring. The electrode's signal was then recorded using Spike 2 (version 6.03, Cambridge Electronic Design) on a Power 1401 acquisition system (Cambridge Electronic Design), stored on the hard drive. A manual record of the penetration locations on the intrinsic signal optical imaging maps was taken to confirm the results of the electrophysiological studies. Offline, the electrophysiological data was further investigated using the Spike 2 analysis software package. 


\subsection{Experimental Stimuli}

In the experiments presented here (Chapter 4), the visual stimuli were presented on a computer monitor (Barco Calibrator V VCID-421, Belgium) 57 inches away from an anesthetized monkey (at this distance 1 degree of visual angle spans 1 inch). A custom-made Delphi language program generated two types of stimuli: achromatic orientation bars (ABar) and monochrome colored orientation bars (CBar). The ABar stimulus consisted of computer generated lines with cell-preferred pixel widths and lengths. Potential stimulus orientations began at $0^{\circ}$ (a horizontal line) and increased in $11.25^{\circ}$ intervals up to and including $168.75^{\circ}$, for a total of 16 base stimuli. Each orientation could be displayed in one of five possible luminances: 13, 19, 25, 31, or $37 \mathrm{~cd} / \mathrm{m}^{2}$. A 'blank' (i.e. no stimulus present on the screen) was used as an additional control stimulus to characterize spontaneous/base level activity present within the cortex. All stimuli were presented randomly. In total, there were 85 (80 bars of various orientations and luminances levels and 5 blanks) distinct stimulus conditions within ABar.

On the other hand, the CBar stimuli consisted of one of the 16 possible orientation lines ( $0-168.75^{\circ}$ in $11.25^{\circ}$ increments) of optimal width and length displayed in one of the 12 possible “colors” (teal, cyan, blue, blue-violet, violet, magenta, pink, orange, orange-yellow, lime, green, and white) at one of the five possible luminance values $\left(13,19,25,31\right.$, or $\left.37 \mathrm{~cd} / \mathrm{m}^{2}\right)$. The psychophysical interpretation of a hue (the perceived color) is dependent on the luminance of the stimulus. In total, there were 960 distinct possible CBar stimuli: 900 colored lines at variable orientations and luminances and 60 "blanks.” However, this particular stimulus set was presented in a pseudo-random manner. In preliminary recordings (Chapter 4), adaptation appeared to affect a cell's firing pattern: constant presentation of the same orientation/color stimulus led to a decrease in neuronal response and spiking activity. To avoid this complication, the preferred angle of the cell (as determined by analysis of previous stimuli sets) was presented, followed by three non-preferred angles, then the preferred angle again. The cycle would then repeat. The colors and the luminance values remained randomized and the set continued until all required color/orientation/luminance combinations had been displayed to the cell.

For both stimulus sets, each particular visual stimulus was presented for $200 \mathrm{msec}$ followed by 200 msec of a black screen, then the next random stimulus appeared. These 200 mslength windows were short enough to allow rapid and successive stimulus presentations, maximizing the data gathered in a short period of time. "Holding” a neuron over long periods 
can be difficult, making it paramount to record electrophysiology efficiently; rapid stimulus presentation is one method that increased this efficiency. 


\subsection{Histology}

Histological methods can be an invaluable tool for studying the properties of cortical tissues and directing future experiments. Several various indicators, markers, "stains", and/or tract-tracing chemicals were employed during the course of data collection in the hopes of better understanding the functional circuitries and organizations of V2 cortex. The histological data further provides an anatomical correlation of a neuron's stimulus preferences and spiking characteristics, adding an investigatory tool to comprehensively confirm post-hoc electrode placement, stripe location, and V1/V2 border proximity.

\subsubsection{Vascular Perfusion}

In terminal experiments, electrode lesions $(7 \mu \mathrm{A}, 7 \mathrm{~s})$ were made (to aid in tissue realignment), followed by a lethal intravenous overdose of sodium pentobarbital (116mg/kg). After the EEG signal had flattened and EKG and respiration rate had both slowed and ultimately ceased, the body was perfused intracardially with physiological saline rinse (2L of $0.9 \% \mathrm{NaCl}$ ) immediately followed by a 2-3\% paraformaldehyde fix in $\mathrm{NaPO}_{4}$ buffer. The brain was removed, sectioned parallel to the V1/V2 border, and allowed to sink in 30\% sucrose. The tissue was sectioned into 30-50 $\mu \mathrm{m}$ slices parallel to the cortical surface for further histological processings. Post-processing, the slices were aligned using Adobe Photoshop CS2 (Adobe Systems Incorporated, San Jose, California) according to cytological patterns, surface vessel landmarks, and electrode lesions.

\subsubsection{Cytochrome Oxidase (CO)}

As previously outlined (Section 1.1.2), $\mathrm{CO}$ is an endogenous metabolic marker found in mitochondria that can indicate differing cytoarchitecture in both V1 (blobs) and V2 (stripes) (Wong-Riley, 1989). At the conclusion of a successful series of electrophysiology experiments and subsequent brain-tissue harvesting, the series of cortical sections were processed for CO histochemistry; initially two differing formularies were used for the processing. To aid in the reconstruction of the tissue, the uppermost layers of the cortex were stained with cresyl violet to aid in vessel and cytological landmark recognition. Ultimately, there was little discernible difference between the results of the two mixtures. The results of individual cortices were somewhat varied in quality, but the difference between CO-dense and CO-poor areas was 
apparent; however being able to discern between thick and pale stripes in V2 could often be subjective. The properties of neurons varied differently between thick and thin stripes: colorpreferring cells organized in the thin stripe and cells within the thick stripe are arranged by orientation preference. Due to this ambiguity that could arise with CO results, alternate methods of labeling the architecture of the visual cortex were also investigated.

\subsubsection{CAT-301}

CAT-301 is a monoclonal antibody that recognizes the chondroitin sulfate proteoglycan located on the extracellular surface of mammalian neurons. Currently there is no definitive role for this particular proteoglycan, but it expressed late in development (Fryer et al., 1992). When used to process macaque striate and extrastriate cortices, areas most associated with magnocellular pathway are immunoreactive. Layers 4B and 4C of V1, which receive inputs from the magnocellular LGN, show reactivity to CAT-301. Compared with the thin stripe, the thick stripe area of V2 is also immunoreactive to CAT-301, providing more specific stripe identification than CO (Deyoe et al., 1990) (when processed with CO, V2 thick and thin stripes can sometimes be ambiguous). Experiments were conducted regarding the feasibility of using CAT-301 to aid in the demarcation of the stripes within V2. Unfortunately, the additional immunohistology did not add much information to the investigation of our V2 cytoarchitecture. The observed results were poor with relatively faint CAT 301 signals in all cases and CAT301 immunohistology was abandoned in favor of focusing on interpreting the results solely from CO.

\subsubsection{Tract tracing}

In addition to the histologic investigations, tract-tracing experiments were also implemented in an attempt to better understand the function of similar clustered cells and the genesis of cells with multiple preferences. These experiments utilized fluorescent tracers, injected by iontophoresis. Typical injection settings consisted of $7 \mathrm{~s}$ on/off pulses of 2.5-5 $\mu \mathrm{A}$ over 10 minutes at a depth of $\sim 500 \mu \mathrm{m}$. Initially these injections were delivered $\sim 12$ hours before delivery of euthanasia, but later experiments injected the tracers a week before the terminal procedure. Additional electrolytic lesions aided in post-hoc cortical reconstruction.

One of the retrograde tracers used was Hydroxystilbamidine (trade name FluorGold). After injection, retrograde tracers are taken up by neuronal terminals and transported back to the 
soma. The fluorescent dye labels the axons and cell body of all neurons it enters, indicating all processes within the area of injection. With retrograde tracers, it is possible to map the projections coming into the area of interest. Two anterograde tracers, micro-ruby and microemerald, were also investigated during the course of this dissertation. As the name might suggest, anterograde tracers enter the body of the neuron and label axon projecting away from the injection sight. Using antero- and retrograde tracers within the same area can indicate the projection coming in and going out of an area. If it were possible to focus both types of tracers within a domain, the pathways associated with the cluster of neurons could be mapped. This manner of investigation could suggest the pathways associated with the domain and the mixed preference cells within. Regrettably these experiments did not yield clear results, with generally weak fluorescence observed in most cases. To ensure that the tract tracing dyes had been appropriately delivered, an anti-FluoroGold monoclonal antibody was used on the cortical tissue after tracer delivery. Immunoreactivity results indicated that the uptake of the tracers had occurred, but it was incomplete and did not fully disseminate through the neuron and its projections. Further investigations were not conducted due to these repeatedly unsuccessful attempts. 


\subsection{V1/V2 Border Determination}

As previously mentioned, electrode penetrations were guided and documented against surface vessel images of the cortical area. To ensure that penetrations were in V2, for example, rather than in $\mathrm{V} 1$ the V1/V2 border was determined by one or more of three possible mapping methods.

The first method, intrinsic optical imaging, provided high resolution functional maps of ocular dominance and/or orientation preference (Section 5.3). Ocular dominance columns, for example, are only present in V1, not V2 (Figure 5-2, left and center panels)), and therefore can be used to preemptively determine V1/V2 border in vivo prior to electrode placements. Similarly, the overall arrangement and size of imaged orientation domains are different in V2 from V1 (Figure 5-2, right panels) and can also be used as areal identifiers.

The second method, comparative RF mapping, used the documented differential characteristics and retinotopic progression of receptive fields (RF) of the neurons to determine V1/V2 border location (Figure 5-3). Compared with RFs of V2 cells, RFs of V1 cells tend to be smaller, and have more pronounced ocular dominance. Furthermore, progressive penetrations in an anterior direction that cross the border will show a reversal in the direction of progression in visual space representation when the border (vertical meridian, VM) is reached. The characteristics RFs of V1 and V2 can be determined using electrophysiology, and ultimately used to determine the location of the V1/V2 border.

The third method, histology, provided a post-hoc determination after the completion of experiments. The processing and staining of tangential tissue sections using cytochrome oxidase histology can delineate the thin-pale-thick-pale striping pattern of V2 compared with the blob/inter-blob punctuate pattern of V1 (Wong-Riley, 1989). At that conclusion of an experiment, the cortical area was marked with electrode lesions and the animal was perfused (Section 5.6.1) then processed with CO (Section 5.6.2). Images of the stained slices were aligned in Photoshop (Adobe Systems Incorporated, San Jose, CA) and correlated with surface vessel images (Figure 5-4). 


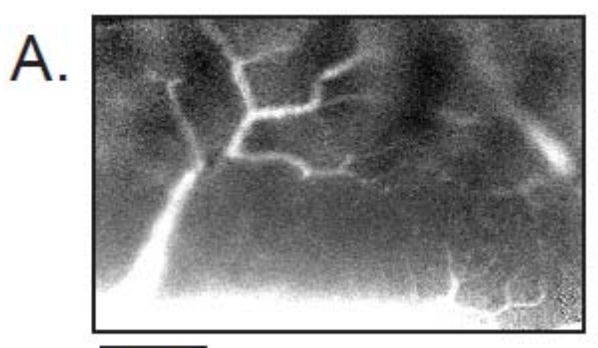

$1 \mathrm{~mm}$

B.
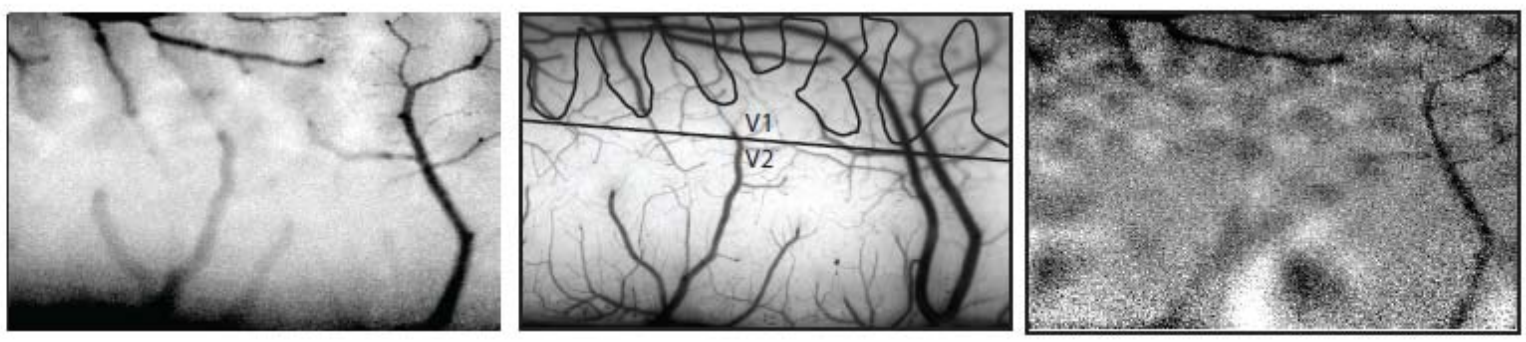

Figure 5-2. V1/V2 border can be determined by optical imaging of ocular dominance and orientation preference. Optical imaging of the visual cortex can be used to map the areas of V1 and V2. V1 neurons organize into ocular dominance 'columns', i.e. long linear domains that abut the V1/V2 border, each with a preference for one eye input. In contrast, V2 neurons receive input from both eyes (and evoke no dominance columns). By determining the cortical regions which have a preference for one eye or the other, it is possible to map the V1/V2 border. Differential mapping (subtracting the summed images obtained from L eye stimulation from the summed images obtained from $\mathrm{R}$ eye stimulation) yields a functional map of ocular dominance (left panels). Darkest pixels in functional maps represent L eye dominance; lightest pixels represent R eye dominance. Average grey pixels represent no dominance. Functional maps are shown superimposed over vessel artefacts that can be correlated with surface vessel map (left panels). Center panels show traced dominance columns superimposed over vessel images that can be used as precise landmarks in experiments. Right panels show functional maps of orientation preference for the same cortex, and pronounced differences in domain pattern and size in V1 compared with V2 may also assist in the determination of V1/V2 border. Darkest domains indicate horizontal preference, lightest domains indicate vertical orientation nreference C.ases shown are exneriments 080707 (A) and 081009 (B) 


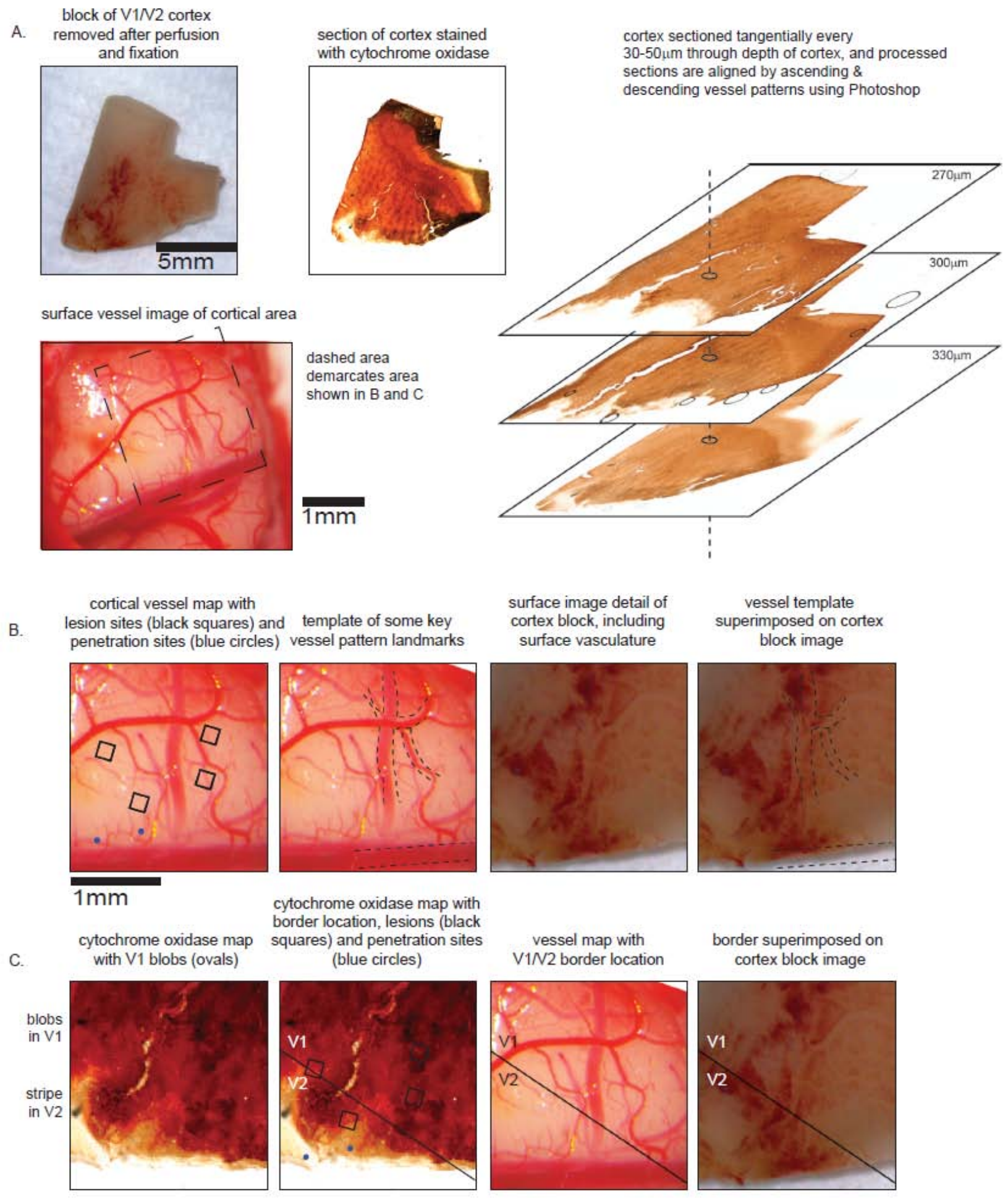

Figure 5-3. V1/V2 border can be determined by histology and penetrations can be mapped to either V1 or V2. At the conclusion of a series of experiments, small current injections produce punctuate lesions in the cortex. This area is then harvested and tangentially sectioned on a microtome. The slices are then processed with histochemical agents such as cytochrome oxidase, demarcating cortical architecture such as V1 blobs and the striping pattern of V2. The slices are then aligned using the vessel patterns and lesions. Electrode penetrations are co-localized on the resulting maps (e.g. blue dots), confirming the areal location. Case shown is 070918. 
A.

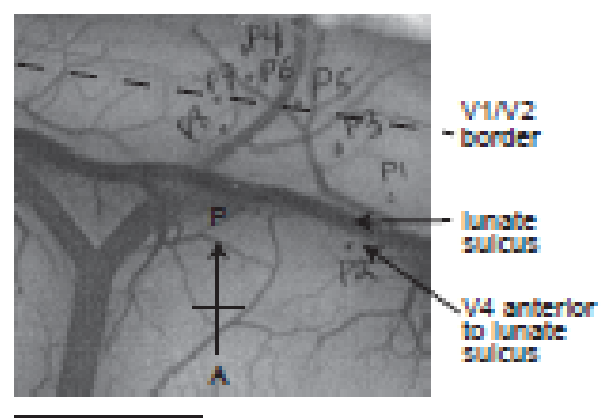

$1 \mathrm{~mm}$
B.

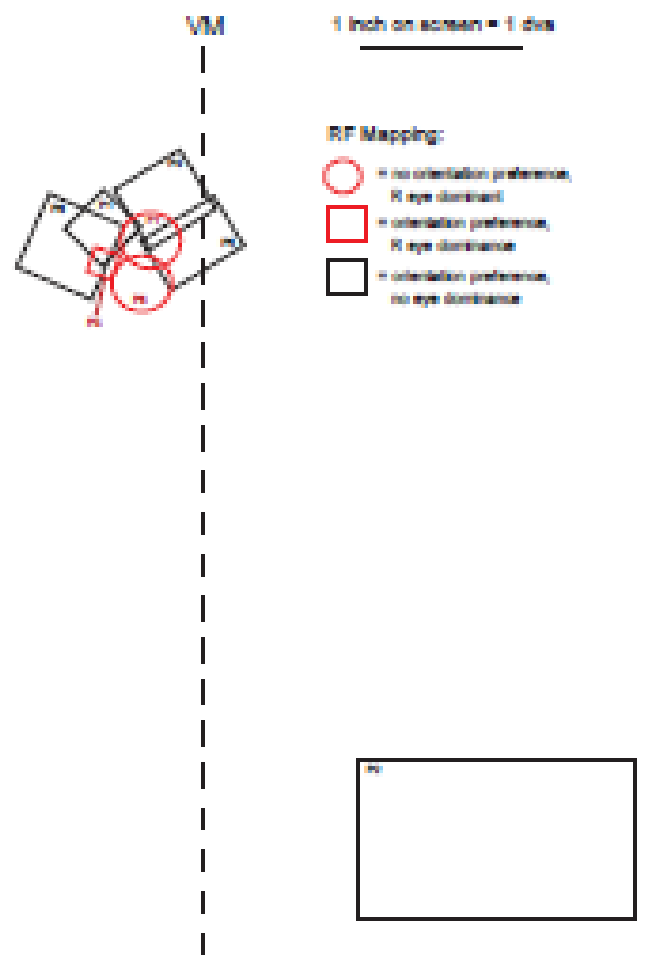

Figure 5-4. V1/V2 border can be determined by receptive field progression. To determine the V1/V2 border, it is possible to examine the characteristics and retinotopic progression of receptive fields (RF) associated with targeted sampling of cortical cells, A. Cells at sites P1-P8 are initially hand-tuned to determine RF size orientation preference and the presence of ocular dominance. Extent and characteristics of RFs (refer legend) are mapped, B, in their relative retinotopic positions, as recorded on a tangent screen 57" from each eye. Cells exhibiting ocular dominance (P4, P6 and P7) and with an RF size $<0.25$ dva (P4) are typically localized in V1 while cells with a larger RF and no ocular dominance (P1, P3, P5, P8) reside in V2. Very large RFs of $\sim 2$ dva are typically seen in V4 (P2). By characterizing multiple RFs and associating them with a vessel map, it is possible to determine the V1/V2 border. In addition, V1 and V2 cells that are closer to the V1/V2 border will have RFs closer to the retinotopic vertical meridian (VM) that is represented along the V1/V2 border. Visual space is functionally represented across V1 and V2 by a mirror asymmetry in relative retinotopic progression, with respect to the V1/V2 border. Thus as V2 cells are sampled progressively closer to V1/V2 border, they will have RFs that are progressively closer to the VM (e.g. compare locations of P1 to P3 to P5). When the V1/V2 border is crossed, neuronal sampling begins in V1 and the the progression in visual space reverses (e.g. compare locations of P6 vs P4). Example RFs and penetrations shown from experiment 071018. 


\subsection{Visual Evoked Potential (VEP)}

Scalp visual evoked potentials (VEP) are electrophysiological field recordings that can provide a gross indication of visual cortical functions that may be used to supplement higher resolution (but invasive) single unit electrode recordings (Kulikowski et al., 2002). This technique has been documented successfully in non-human primate research (Previc, 1986). Constraints on temporal (i.e. modulations of cellular activity) and spatial (i.e. single cell) resolution currently limit the effectiveness of VEP in an examination of V2 neurons with mixed properties and cluster interactions at a single cell level, however an indirect use of this technique was important in data collection.

Finding the proper balance of anesthesia was a difficult issue that arose continuously during electrophysiology experimentation. In general, anesthesia can have an effect on the "normal" functioning of the brain, leading to altered or even erroneous responses as observed through single electrode recordings. Monitoring the levels or "depth" of anesthesia can be especially pertinent when doing vision research - how do we know if the animal can see the stimulus, whether due to improper eye focus, obstruction or inadequate levels of anesthesia? In an effort to address these concerns, VEP electrode recordings provided supplementary electrophysiological information that, when correlated with physiologic markers such as heart rate and blood pressure, provided an insight to what the animal "saw" - brain activity in response to the stimuli confirms not only a "responsive" brain, but that the setup (e.g. contacts, visual focus) were sufficient for further experimentation.

In humans, a VEP has a characteristic peak at $100 \mathrm{msec}$ after stimuli presentation, known as P100, and is thought to arise from activity in the striate and parastriate visual cortices (Chiappa and Ropper, 1982a, b; Grundy, 1983). The P100 can be effected by anesthetic agents: isoflurane increases P100 latency and decrease the amplitude (Chi and Field, 1986), fentanyl (an opioid similar to sufentanil) decreases the amplitude (Chi et al., 1987a), fentanyl-nitrous oxide yields an increase in latency (Chi et al., 1987b), and propofol has no significant effect (Ver Hoeve et al., 1999). The resolution acuities observed in Macaca fasicularis are comparable to humans (De Valois et al., 1974), suggesting that VEP would be an appropriate candidate to examine the effects of the anesthetics that were used in our experiments on macaque monkeys. 


\subsubsection{Materials and Methods}

After an appropriately sized area of skin was shaved and cleaned at the nasion and inion of the animal subject, one of two scalp electrodes with a small amount of electrode gel was attached at either point (this particular setup was especially chosen as it did not interfere with the chamber or electrode preparation). Sufficient scalp contact was determined by the measurement of electrical impedance (typically $\leq 15 \mathrm{k} \Omega$ ). Animals were artificially ventilated and also monitored via EEG, EKG, respiration rate with end-tidal $\mathrm{CO}_{2}$, oximetry, blood pressure, and pulse-based heart-rate monitoring (Cardell MAX-1, Sharn Veterinary, Inc., Tampa, FL). All procedures were conducted under approved protocols sanctioned by West Virginia University Animal Care and Use Committee and Office of Laboratory Animal Resources.

Two different luminance stimuli were presented to the animal subjects during VEP acquisition: a square-wave, pattern-reversal checkerboard and a sinusoidal checkerboard. A measure of anesthetic depth was assessed using the sinusoidal stimuli at a spatial frequency of 1 degree of visual angle (dva), and a temporal frequency of $1 \mathrm{~Hz}$. To examine visual acuity (as a measure of optics), the VEP stimuli were reversing checkerboard patterns presented at $1 \mathrm{~Hz}$, with spatial frequencies varying from $0.05-1.5$ dva. VEPs recorded from these stimuli were subjected to a Fourier analysis and the signal power at the reversal frequency was quantified. Amplitude and latency of P100 were correlated with the animal's physiology, such as heart rate and blood pressure.

\subsubsection{Results}

Through the use of VEP, it was possible to further monitor the anesthetic depth and ensure the necessary visual acuity of multiple animal subjects (Figure 5-5). By observing the modulations in the height of the P100 peak in association with the heart rate and blood pressure, we could observe the affect of the anesthesia on the animal. If the level of anesthesia was too deep, a lengthening of P100 latency and flattening of the peak were noted. By determining the critical anesthetic level for each experiment (and each animal), it was possible to ensure an adequate level of anesthesia for the animals well-being and success of the experiment. This technique was able to indicate the individual physiology for each macaque, allowing the customization of anesthesia on an experiment-by-experiment basis. It was also possible to use VEP to check an animal after an extended period of time or at the end of an experiment to 
validate the optics and anesthetic depth. Through an examination of P100, a drop in VEP activity was correlated with a decrease in blood pressure and not in heart rate. This information provided a baseline for electrophysiology and anesthesia adjustments based upon the animal subject's blood pressure. The acuity investigation indicated that the animal's vision would be sufficient for further investigation.
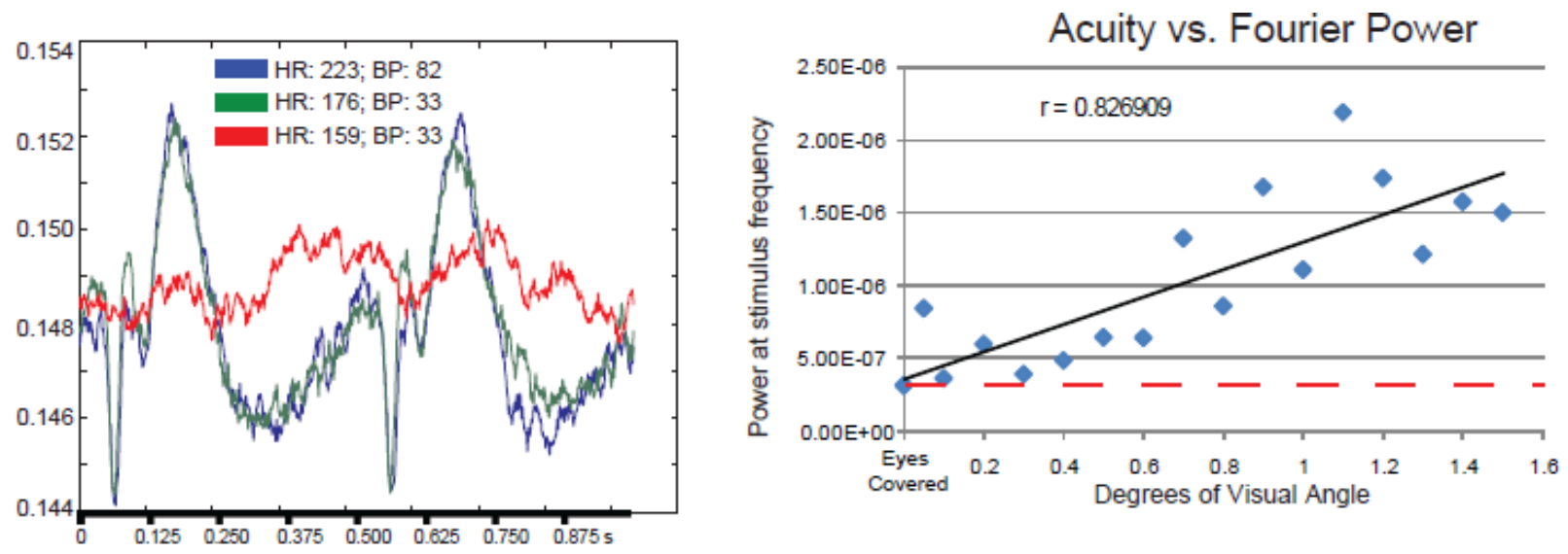

Figure 5-5. Visual evoked potentials used to determine anesthetic depth and visual acuity. Through the recording and interpretation of visual evoked potentials (VEP), changes in recorded activity can be observed through anesthesia manipulation and blood pressure. Visual acuity of the animal and the optics of the experiment can be confirmed through spike amplitude modulations in the VEP. 


\subsection{Baseline and Latency Calculations}

To examine a neuron's temporal data, a 2D histogram was used as this method afforded a more comprehensive view of a cell's temporal data in addition to Poisson's Surprise and Spike Density Function (SDF) to examine neuronal latency. The background responses (or noise, baseline) were defined as the mean firing rate during the blank presentation (Priebe et al., 2006). This particular time frame was chosen because it is representative of activity when there is no stimulus on the screen and will be compared to activity when any stimulus is present.

All recordings must have met certain criteria before inclusion in a data set. During a 25 trial experiment, 250 significant spikes must have occurred if a cell was to be further analyzed (i.e. there must be at least ten significant spikes in one complete stimulus set presentation) (Anzai et al., 2007). This ensured that the cell was responding adequately to the stimulus before a further analysis was executed. Complex/simple cell is another possible concern when determining which neurons to include for analysis. According to Hubel and Wiesel, a simple cell is defined when the stimulus yields unsustained responses of the same type across the receptive field (i.e. weaker or lack of spiking in the inhibitory domain, with stronger activity in the excitatory region). However a properly oriented line swept across the entirety of the receptive field yielding a well-sustained barrage of impulses is indicative of a complex cell (Hubel and Wiesel, 1968). Since simple and complex cells can yield different spiking activities as a result of the stimulus location within the receptive field, the field must be properly mapped and the stimulus must be positioned over the excitatory region of the field to ensure that the recorded activity is truly representative of the neuron's response to the stimulus.

A significance threshold, calculated as two standard deviations (of baseline variance) greater than the baseline ensured that only activity significantly greater than baseline (the activity when no stimulus is present) was further investigated (Smith et al., 2006). In other words, the significance threshold (2 Std Dev > baseline) was subtracted from all recordings before any additional analysis was run (Anzai et al., 2007). The remaining activity after threshold subtraction was defined as significant spiking activity.

The significant spikes resulting from each stimulus were summed and these totals were smoothed via averaging the two surrounding columns. This moving window of averaging was an attempt to eliminate "noise" and extract real trends and patterns from the spiking activity. A particular stimulus's response must have been at least the average stimulus response $+90 \%$ 
(190\% of the mean) and also have the maximum summed responses (of all stimuli) to be considered that neuron's preferred stimulus (i.e. color or orientation). The extent of a continuous area of preference (i.e. multiple "preferences” or "tuning width”) was defined as all other stimuli with an amplitude $>190 \%$ of the average response but a no-less-than $10 \%$ difference in amplitude than the maximum (Ito and Komatsu, 2004). If a neuron possessed multiple preferences or the maximum was not $>190 \%$ of the average, the cell was defined as exhibiting no preference. This did not preclude the neuron from further analysis, however; the stimulus that yielded the maximum number of spikes was used in all calculations that refer to the preference.

As latency was a primary temporal characteristic examined in this dissertation, an exact definition was necessary. Latency was defined as the first significant spike (after baseline subtraction) of the cell to its preferred stimulus. The preferred stimulus was always used to determine latency to limit variability, since the latency of a neuron increases when presented with a non-optimal orientation bar (Celebrini et al., 1993). The length of a response was determined from the first to last significant spike, and all of these calculations were derived from the multi-trial average.

The peaks, or maxima, of a cells response were investigated using First and Second Derivative Tests based on Fermat's theorem. In essence, if a bin had significant spikes (> std dev baseline), and the previous two bins and subsequent two bins had less significant spikes, that bin was at a maximum (peak). If more than one maxima was present, the smaller (local) maxima could not have an amplitude within $90 \%$ of the bin with the most peaks (global maximum) otherwise no dominate maximum existed. Peaks were only investigated within the cell's preferred stimulus.

Previous studies indicate that certain criteria also exist for excluding potential data, since latency (and other temporal properties) outliers may occasionally arise due to bursty, spontaneous activity. For instance, if a calculated latency was below $50 \mathrm{msec}$ (significantly below previously recorded times (Munk et al., 1995)) or if the latency was greater than $200 \mathrm{msec}$ (beyond the time window when the stimulus was present), then the results from that cell were excluded. Aberrant spike clusters (i.e. a cluster of spikes firing rapidly with a significantly higher amplitude and rate as determined by the 10 spike minimum) were also ground for exclusion. Poisson's Surprise was also implemented to determine the "normal" spiking activity and to locate areas of above “normal” spiking. Any severe change in the animal’s physiology (i.e. heart 
rate, EEG level, etc) also potentially yielded data that was not be used since the alterations in physiology could have resulted in unreliable recordings. Such changes were observed and noted during the procedure and precluded the data from analysis. 


\subsection{References}

Bergman SA (1999) Ketamine: review of its pharmacology and its use in pediatric anesthesia. Anesth Prog 46:10-20.

Bianchi SL, Tran T, Liu C, Lin S, Li Y, Keller JM, Eckenhoff RG, Eckenhoff MF (2008) Brain and behavior changes in 12-month-old Tg2576 and nontransgenic mice exposed to anesthetics. Neurobiol Aging 29:1002-1010.

Chen LM, Heider B, Williams GV, Healy FL, Ramsden BM, Roe AW (2002) A chamber and artificial dura method for long-term optical imaging in the monkey. J Neurosci Methods 113:41-49.

Chi OZ, Field C (1986) Effects of isoflurane on visual evoked potentials in humans. Anesthesiology 65:328-330.

Chi OZ, McCoy CL, Field C (1987a) Effects of fentanyl anesthesia on visual evoked potentials in humans. Anesthesiology 67:827-830.

Chi OZ, Moroz A, Field C (1987b) The effects of nitrous oxide-fentanyl anesthesia on visual evoked potentials in humans. Anesth Analg 66:S27.

Chiappa KH, Ropper AH (1982a) Evoked potentials in clinical medicine (second of two parts). N Engl J Med 306:1205-1211.

Chiappa KH, Ropper AH (1982b) Evoked potentials in clinical medicine (first of two parts). N Engl J Med 306:1140-1150.

De Valois RL, Morgan H, Snodderly DM (1974) Psychophysical studies of monkey vision. 3. Spatial luminance contrast sensitivity tests of macaque and human observers. Vision Res 14:75-81.

Deyoe EA, Hockfield S, Garren H, Van Essen DC (1990) Antibody labeling of functional subdivisions in visual cortex: Cat-301 immunoreactivity in striate and extrastriate cortex of the macaque monkey. Vis Neurosci 5:67-81.

Euliano TY, Gravenstein JS (2004) Essential anesthesia: from science to practice. Cambridge, UK: Cambridge University Press.

Frostig RD, Lieke EE, Ts'o DY, Grinvald A (1990) Cortical functional architecture and local coupling between neuronal activity and the microcirculation revealed by in vivo highresolution optical imaging of intrinsic signals. Proc Natl Acad Sci U S A 87:6082-6086.

Fryer HJ, Kelly GM, Molinaro L, Hockfield S (1992) The high molecular weight Cat-301 chondroitin sulfate proteoglycan from brain is related to the large aggregating proteoglycan from cartilage, aggrecan. J Biol Chem 267:9874-9883. 
Fujinaga M, Maze M (2007) Neurobiology of nitrous oxide-induced antinociceptive effects Molecular Neurobiology 25:167-189.

Grinvald A, Lieke E, Frostig RD, Gilbert CD, Wiesel TN (1986) Functional architecture of cortex revealed by optical imaging of intrinsic signals. Nature 324:361-364.

Grundy BL (1983) Intraoperative monitoring of sensory-evoked potentials. Anesthesiology 58:72-87.

Harrison NL, Simmonds MA (1985) Quantitative studies on some antagonists of N-methyl Daspartate in slices of rat cerebral cortex. Br J Pharmacol 84:381-391.

Hubel DH (1957) Tungsten Microelectrode for Recording from Single Units. Science 125:549550.

Jevtovic-Todorovic V, Todorovic SM, Mennerick S, Powell S, Dikranian K, Benshoff N, Zorumski CF, Olney JW (1998) Nitrous oxide (laughing gas) is an NMDA antagonist, neuroprotectant and neurotoxin. Nat Med 4:460-463.

Kruger J, Aiple F (1988) Multimicroelectrode investigation of monkey striate cortex: spike train correlations in the infragranular layers. J Neurophysiol 60:798-828.

Kulikowski JJ, Robson AG, Murray IJ (2002) Scalp VEPs and intra-cortical responses to chromatic and achromatic stimuli in primates. Doc Ophthalmol 105:243-279.

Lambert S, Arras M, Vogt KE, Rudolph U (2005) Isoflurane-induced surgical tolerance mediated only in part by beta3-containing GABA(A) receptors. Eur J Pharmacol 516:2327.

Livingstone MS (1996) Oscillatory firing and interneuronal correlations in squirrel monkey striate cortex. J Neurophysiol 75:2467-2485.

Miner JR, Burton JH (2007) Clinical practice advisory: Emergency department procedural sedation with propofol. Ann Emerg Med 50:182-187, 187 e181.

Nowak LG, Munk MH, James AC, Girard P, Bullier J (1999) Cross-correlation study of the temporal interactions between areas V1 and V2 of the macaque monkey. J Neurophysiol 81:1057-1074.

Previc FH (1986) Visual evoked potentials to luminance and chromatic contrast in rhesus monkeys. Vision Res 26:1897-1907.

Ries CR, Puil E (1999) Ionic mechanism of isoflurane's actions on thalamocortical neurons. J Neurophysiol 81:1802-1809. 
Ver Hoeve JN, Danilov YP, Kim CB, Spear PD (1999) VEP and PERG acuity in anesthetized young adult rhesus monkeys. Vis Neurosci 16:607-617.

Veselis RA, Reinsel RA, Feshchenko VA, Wronski M (1997) The comparative amnestic effects of midazolam, propofol, thiopental, and fentanyl at equisedative concentrations. Anesthesiology 87:749-764.

Wong-Riley MT (1989) Cytochrome oxidase: an endogenous metabolic marker for neuronal activity. Trends Neurosci 12:94-101.

Xie Z, Dong Y, Maeda U, Moir RD, Xia W, Culley DJ, Crosby G, Tanzi RE (2007) The inhalation anesthetic isoflurane induces a vicious cycle of apoptosis and amyloid betaprotein accumulation. J Neurosci 27:1247-1254. 
CHAPTER 6: General Discussion 


\subsection{Overview}

A number of previous studies have identified and characterized the functional clustering of neurons in Area V2 cortex. Functional domains with overall preference for color or orientation, for example, are well documented, although the possible role for this clustering remains elusive. Are domains simply efficient organizations that arise from early cortical development and which have no significant function (beyond wirelength minimization)? Or alternatively, could the grouping of similarly preferring neurons aid the processing of visual stimulation via some computational mechanism? In addition, investigators have also identified visually responsive neurons in V2 with preference for more than one stimulus attribute, although so far this has not usually been associated with or interpreted in relation to clustered organizations per se.(Kim et al., 2000; Ramsden et al., 2001; Ohki et al., 2005; Chen et al., 2007; Yacoub et al., 2008). In this dissertation, a model with V2-like clustered functional cortical organizations was devised and implemented to investigate the putative processing advantages associated with functional organizations of the scale and relative displacements found in macaque V2. If color-preferring functional domains abut orientation-preferring functional domains, and these organizations do play a functional role in combined color-orientation integration, it will be important to understand how these relative domain arrangements influence the temporal evolution of neuronal response. A second part of the dissertation characterized and compared single neural recordings in anesthetized monkey V2 cortex in response to both single attribute (e.g. colored but not oriented, or oriented but not colored) vs. combined attribute (oriented and colored) visual stimulus presentations.

This dissertation has sought to investigate the functional purpose of neuronal clustering and their putative role in the generation of neurons with multiple preferences in V2. The possible advantages of domain organization as a structural aid to assist integration of function may also exist in other areas beyond V2, although other cortical areas were not explicitly investigated here. Indeed, other areas could have been selected for investigation here for the exploration of a possible functional domain role. However, the initial investigation of Area V2 may be especially appropriate however, due to the close proximity of orientation- and color-preferring domains, and the relatively large size and ubiquity of these domains. Importantly, these domains comprise neurons with relatively simple selectivity characteristics that are readily accessible for both single unit electrophysiological sampling and high resolution functional imaging. These 
characteristics provide scope for testable preference differences. Thus, the macaque cortical Area V2 provides an excellent in vivo model to examine functional clustering roles. Additionally, since the domains of this area have been especially well-documented in the macaque monkey, it is possible to develop analogous computer models with credibly realistic features. These models have potential to help better understand existing in vivo experimental data and may guide the formulation of new hypotheses that can be investigated in future animal experiments.

\subsubsection{Computational network modeling}

Surprisingly, despite the growth in understanding of the distribution and classification of domain preference in V2 over the last twenty or more years, there has been no known attempt to specifically model and investigate the consequences of the domain arrangements and potential integrative actions. The modeling data presented in this dissertation indicated that a single preference domain (e.g. orientation) could lead to a significantly increased probability of terminals with that preference in the immediate vicinity of the domain. Thus neighboring neurons of otherwise different preference characteristics (e.g. color) would have a higher probability of receiving additional inputs from neurons within the (orientation) domain. This suggests that even the presence of a hypothetical isolated domain could bring about the higher probability of integrative function in the immediate vicinity of the domain. In macaque cortex (and probably cortex in other species as well) domains are unlikely to exist in isolation. To investigate the influence of multiple domains on the possible enhancement of functional integration a dual domain model was implemented with one domain preferring one stimulus attribute (e.g. an orientation) and a second domain preferring a different attribute (e.g. color), as reported in V2. Adding a second domain further enhanced the probability of integration of preference (compared to the single domain model), especially in the region between the domains. As an alternative to comprehensive modeling of many domains, an additional model was developed that instead tested how pairs of domains might operate as functional integrators in a biologically inspired multi-domain map (Model 2). The data obtained from Model 1 (paired domains) was used to obtain sufficiency criteria. These results indicated a statistical likelihood that V2 stripe proximity is more than sufficient for a significant and comprehensive integration of terminals from most types of color- and orientation-preferring domain pairs. In Area V2, this kind of pooling may be especially pronounced due to the systematic and repetitive sequences of 
different domain types across the cortex. Model 2 results support the notion that the spacing and width of thin, thick and pale stripes of V2, and the organization and large breadth of the domains within the stripes allow for comprehensive inter-areal integration of color and orientation preferences.

The combined results from the two models, representing the functional organization of neurons in macaque V2, suggest a geometric propensity for domain terminal integrations in V2. If the highest probability for integration of differently preferring terminals occur between (but outside) the domains then one would expect a gradient in prevalence of color-orientation neurons between adjacent color and orientation preference domains in in-vivo recording experiments. Color-orientation selective neurons have been noted at the border of orientation- and colorpreferring stripes in macaque V2, although the details of this distribution of color-oriented preferring neurons remain to be elucidated (Roe and Ts'o, 1995). While other mechanisms of integration are feasible (e.g., inter-areal convergence, or integration in V1, see discussion in Chapter 3), the prediction and observation of cells with multiple modality preferences is consistent with a mechanism of common inputs from different preference domains. Furthermore, the joint probability of color/orientation inputs may be further enhanced functionally because of increased local recurrence (Douglas et al., 1995). However the models that were applied excluded detailed dendritic sampling parameters and known basal dendritic spread in macaque V2 (Elston and Rosa, 1997) would be expected to diminish punctuate peaks while preserving any spatially co-extensive peaks.

When multiple color and orientation domains were aligned and evenly distributed in plausible stripe-like configurations (as observed in the second model), the model more closely represented the multiple potential interactions of many domains as would occur in macaque V2. The Model 2 results suggest that the combination of stripe dimension and domain dimension yields a high probability that all color domain classes will be in sufficiently close apposition to all orientation domain classes affording a relatively complete assortment of neurons containing all preference combinations. Thus the spatial scaling of functional structure in V2 appears to be well designed for an efficient neural coding. Yet recent data has shown that other 'non-color' luminance domains may exist within the V2 stripes and the possibility of a more compressed/overlapping representation of color preference domains (cf. even distribution across a model thin stripe compartment) occurring episodically in the stripe (Xiao et al., 2003). This 
was not explicitly tested in these experiments. However, it is reasonable to draw the conclusion that greater overlap of color domains (relative to overall thin stripe territory coverage) could support a balance of orientation/color integration while maintaining 'vacancy' in V2 thin stripes for other stimulus feature mappings (Chen et al., 2005; Wang et al., 2006). This may however require greater duplication of these spatially compressed color modules in order to ensure color modules are sufficiently close to all classes of orientation domain for complete intra-areal integrative representations.

\subsubsection{Characterization of V2 neurons' temporal responses to achromatic- and color-oriented} stimuli results

The in vivo electrophysiology experiments indicated that the addition of color to a preferred (achromatic) orientation stimulus resulted in a diverse modulation of temporal responses of V2 neurons. First, the addition of a preferred color to the stimulus produced a significant change in mean latency overall; nearly two-thirds of the cells displayed a significant increase in time from stimulus presentation to the point of significant increase in neuronal activity. Secondly, the addition of color also brought about a significant increase in mean time to peak firing rate of the cellular response. Finally, a preferred-color, preferred-orientation stimulus generated a significant reduction in the initial, transient phase but preserved the later, sustained response, as observed through a comparison of the mean spiking activities.

These neural response characterizations provide new quantitative data in the anesthetized macaque regarding the differential single V2 neuron response to oriented vs. oriented-colored stimulus conditions. By comparing the neural response to differing stimulus components some authors have drawn conclusions about possible underlying circuitries (e.g. see Anzai and Van Essen, 2007). One possibility is that separate and convergent color- and orientation-dominant pathways from V1 may present temporally distinct input signals onto their recipient color- and orientation preferring V2 domains. If intra-areal connectivity within V2 plays a significant role in the integration of color and orientation, then the passage from color- to orientation-preferring domain or orientation- to color-preferring domain may correspond with specific and predictable changes in the temporal activity signatures recorded from neurons within these V2 domains. The unraveling of such hypothetical temporal sequences and their circuitries will require a very large number of recordings from specifically targeted locations (domain and stripe) in V2. The neural 
recordings obtained and presented in this dissertation are a small set of characterizations made without detailed functional domain and stripe correlates, and so it is not feasible to draw convincing conclusions about the underlying circuitry involved. However, these data nevertheless provide useful introductory characterizations of these neural processes and suggest the presence of some surprising temporal relationships which deserve further investigations in future. One of the properties examined in most detail was response latency, or the time period from stimulus presentation to significant neuronal spiking activity. Although the calculated results of latencies were computed from chromatic/achromatic stimulus presentations to the same cell, the reported times here and the increase in latency coincides with those previously described in different populations of color- and orientation-preferring cells (Munk et al., 1995; Schmolesky et al., 1998). Understanding latencies of neurons in response to stimuli are also thought to be important factors in attention models (Oram et al., 2002) and rapid behavioral responses (VanRullen et al., 2005). A significant increase in a single cell's latency was observed when the preferred color was added to a preferred orientation bar by an average of 16 msec. While it has been reported that "higher" visual cortical levels display longer latencies then those of earlier cortices (Raiguel et al., 1989; Maunsell and Gibson, 1992), previous work had not definitively examined how the same cell's response could be modified in response to a changing stimulus. The results presented here suggest that temporal properties of individual cells are mutable and stimulus-sensitive. A cell possessing two different latencies in response to two independent stimulus attributes might suggest separate pathways of innervation at the level of a single neuron (e.g. a neuron within a pale stripe, or a color-selective neuron). In this scenario, each calculated latency suggested the circuitry information could follow en route to the spiking neuron, allowing us to predict potential methods of "cross-talk" within V2.

In addition to latency, other temporal response measures were investigated in this cell population. Maximum spiking, or the global maximum, was one such type of temporal response examined. The point at which maximum firing occurred increased by an average of $52 \mathrm{msec}$ when a cell was presented with a preferred-color, preferred-orientation stimulus. The significant lengthening of the cell's response until peak spiking activity reinforced the proposed assumptions from the latency calculations: segregated pathways carrying disparate information synapse on a single neuron in V2. Through careful examination of the averaged spiking response over time, significant decrease in the amount of initial spiking activity was also observed. The 
addition of chromatic information yielded an averaged "dampening" of activity during the 100 130 msec time period of the temporal response, which may be the product of inhibitory mechanisms. Since the decreased response was transient - as the remainder of the cell's response was virtually identical - local circuitry could be a candidate for the inhibition resulting in this modification (Douglas et al., 1995). If a cell with mixed preferences receives projections from different functional domains, the decrease in initial spiking but a lengthening of peak spiking activity could reflect the additional time needed for input from the second domain (in this example color) in response to a mixed property stimulus. 


\subsection{Significance of the Results}

\subsubsection{The role of clustered organization, intra-areal connections, and mixed preferences}

Bipartite projections from V1 to V2 (Livingstone and Hubel, 1984; Hubel and Livingstone, 1987; Sincich and Horton, 2002) do not clearly suggest how predominantly separate pathways of color-dominant and orientation-dominant visual information may be effectively integrated at the level of the secondary visual cortex. Several possible designs have been proposed to account for the modulation of a cell's response and the cell's stimulus preference. The two pathways could send projections to a single cell (Roe and Ts'o, 1999) or interact with other cortical regions to yield such modulations. Achromatic information along the magnocellular pathway has been documented to transmit information significantly faster than color along the parvocellular pathway (Maunsell et al., 1999) and this may account for the faster latencies and shorter maximum firing points with an achromatic stimulus. Yet previous work has suggested the mixing of parvo- and magnocellular pathways as early as V1, so a compound stimulus of color and orientation might be expected to evoke a temporal response similar to its fastest component: orientation (Johnson et al., 2004). Since we observed an increase in response time on average, this would suggest that the slower color information dominates or alternatively inhibits the faster orientation component. A third pathway, the koniocellular pathway, also sends projections from S-cones that arrive relatively late into V1 (Cottaris and De Valois, 1998; Casagrande, 1999; Hendry and Reid, 2000). A non-linear summation occurs when the net response of a cell from multiple stimuli is not equal to the sum of the individual stimuli. Recent research has suggested the presence of non-linear summations from some V2 cells preferentially responding to aggregated stimuli comprising multiple orientation bars or alternatively spatially separated color and orientation compound stimuli (Ito and Komatsu, 2004; Hinkle and Connor, 2005; Anzai et al., 2007), suggesting a complicated interaction of multiple cells. The identification of the precise network of neuronal connections needed to fully explain the neuronal responses associated with multiple modality preferences and variable temporal properties therefore remains a difficult challenge especially when using only electrophysiological recording methods. One future approach might utilize targeted electrophysiological sampling of multiple neurons guided by detailed mapping of function at high spatial resolutions. Importantly, the recent development of two photon functional microscopy methods offers the potential to visually identify neuronal preference at two spatial scales: that of the functional domain and that 
of the individual neuron (Ohki et al, 2005). The modeling results imply that architecture and geometry of V2 permits ample intra-domain associations that could yield cells with a color and orientation preference on a single cell supported by the advantage of inter-domain intra-areal connections. The recorded modulations of temporal responses documented from the in vivo data provide an initial characterization of the engagement of the circuits associated with color and orientation integration in V2. That latency is extended on average on the addition of color attributes to an oriented stimulus presentation is intriguing and deserves further investigations in future Nevertheless, the dataset presented here is still relatively small and uncorrelated with detailed high resolution functional domain mapping and so it remains difficult to draw firm conclusions about the possible underlying circuitry from the temporal response characteristics alone. However, with the documented likelihood of local interaction, and the close proximity of clustered organizations of neurons each comprising distinct preferences it is not unreasonable to suggest an intra-areal network may be a likely mechanistic candidate (Levitt et al., 1994; Kiper et al., 1997; Shipp and Zeki, 2002).

The central theme of this dissertation focuses on cluster organization and its putative role in the integration of responses associated with different stimulus attributes. In particular, the experiments described here investigate the possible integration of color and orientation responses at the level of V2, mediated by intra-areal connections within V2. The modeling experiments directly examined cluster interaction: functional domains significantly increase the probability of the co-occurrence of terminals (and most likely the activation of synapses) of different preferences on a singular "integrative” neuron. The in vivo electrophysiology experiments investigated the temporal effects of achromatic/color-oriented stimulus on V2 neurons and among the various observed changes, noted distinct temporal response profiles on average and in particular an increase in latency associated with the addition of color stimulus attributes to an oriented stimulus figure. The modeling results suggest that an intra-areal interaction is a feasible component in the integration of color and orientation within V2. A significant increase in latency on average (as well as the other variations in temporal processing) obtained from the recording experiments indicate that distinct (and intriguing) modulations of temporal properties appear to be associated with color and orientation integration early in the visual area hierarchy. 


\subsubsection{Limitations of Modeling Experimental Design and Interpretation}

Computer modeling is constrained by the practicalities of algorithmic implementations if too much realistic detail is specified in the design then, in turn, it may become increasingly difficult to determine the important mechanisms underlying a particular modeled cause (e.g. a modeled visual stimulation) and subsequent effect (e.g. a modeled electrophysiological response). On the other hand if a model is an oversimplified implementation then crucial network contributions may be significantly reduced or completely overlooked. When attempting realistic computer network modeling of functional circuits of the primate cortex these problems are paramount. What parameters and variables need to be specified? What values should they be assigned? The model presented here is based upon the macaque V2 and its known parameters. It is of note, however, that these parameters are based upon scientific observation but at this point in time are not all inclusive. Currently this model simplifies the cortical neurons and their physiology but does not address all the different possible circuitry that may project to and from V2 neurons. A structural model simplification was arguably required for these first developments of a practical model for putative integrations associated with clustered functional organizations, however. Given the model results and conclusions obtained it may be practical to introduce further appropriate complexities that were not included here. The model used was an isolated, "closed-circuit" network that received no explicit inter-areal feedforward or feedback circuits. Furthermore, individual cell preferences were assigned and only varied based upon cluster interaction. Some network elements were randomized (e.g., cells outside the defined model domains), inconsistent with actual cortical organization in V2 but arguably essential for controlled implementation and interpretation here. Modeling, however, can have many advantages when used in conjunction with in vivo experiments, such as the electrophysiology investigation discussed in this dissertation (Chapter 4). As computer models become increasingly complex and parameter specifications become even more biologically representative, it may be possible to further reduce experimental animal use in some cases. The results of modeling are highly controlled and therefore consistent and efficient, as opposed to the challenging practical complications associated with electrophysiological recordings. Though model parameters can be manipulated in many ways (and potentially obfuscate results), careful modification can create scenarios and experiments not possible in in vivo investigations and grant new insights and future 
directions for continuing research. Currently animal models are still necessary, but the results from these studies can be further understood using computer modeling.

\subsubsection{Limitations of Electrophysiological Experimental Design and Interpretation}

The in vivo cell population data, on the other hand, is a limited set, allowing only tentative conclusions. The complicated nature and technical limitations of electrophysiological studies constrains the number of cells meeting the guidelines to be included in this study. Acceptable recordings require the holding of a visually responsive cell for long periods of time, and the maintenance of stable anesthesia and associated vital signs. Though postulating about the general response of a neuron requires a large population of cells, the changes in the average temporal responses we reported are still of importance. The increase in latency, the lengthening of time until maximum spiking and the decrease in spiking activity during the initial response were all significant on average. Additionally, future latency studies may benefit from validated knowledge of stripe and cluster type, which were not able to be sufficiently determined in the studies described here. The modeling adds some credence to our proposition, outlining the probability and likelihood of intra-areal connections in V2 as a potential mechanism for our observations. Additionally, a similar study of V1 cells and any resulting modifications in temporal response could indicate if our reported effects are localized to V2. A larger data set would provide an opportunity to reinforce the findings and in addition provide more useful detail of the overall distribution of neurons with differential temporal responses. It is also of note that not all cells displayed a similar modulation with the addition of color to an oriented bar stimulus; some cells showed decreases in temporal properties, indicating a heterogeneous pattern of temporal modulation in V2. These variations suggest a complicated interaction of projections and circuitry up-to and within V2, and a thorough statistical interpretation of these intricacies would benefit from a greater set. Interestingly, the sampled neurons appeared to differ in their correlation of paired response measures: not every cell with an increase in latency yielded a longer time to peak firing and vice versa. 


\subsection{Directions for Future Study}

While our computer-based modeling was a relatively simple structural implementation (cf. actual brain circuitry and compared with some other very large scale simulations, e.g. Markrams Blue Brain), further development of the visual cortical model is quite feasible and could be beneficial. Many existing off-the shelf packages however are more geared to small scale neuronal networks rather than sheets of neurons that interconnect "intra-areally" or "interareally”. Topographica (Edinburgh, UK), is one recently developed Python and C++ based simulation program that can emulate the activity of thousands of visual neurons within a cortex (Bednar et al., 2004). Such a program would permit manipulation, testing, and modification of the program code (it is an open-source program) of specific integrative process in macaque V2. Sheets of model cortical neurons could be programmed with specific inputs, outputs, functional maps and overall intra-areal connections. Within these sheets, groups of neurons are associated and can be considered a "super neuron" (i.e. a single function representing many neurons). The super neuron itself is based upon the leaky integrator model (this model accounts for decayed summation of incoming spikes at each synapse). Outgoing spikes are synthesized and calculated from the weighted sum of the inputs with regard to the dynamic spiking threshold. This could provide useful temporal parameters and variables that would allow model comparison of spatiotemporal patterns that could be compared with those spatiotemporal patterns obtained in vivo. The possible inputs include afferents, excitatory lateral, inhibitory lateral, and intracolumnar connections, all of which are lacking from the model presented here (Chapter 3). These factors, although arguably necessary for better understanding of cluster organization and cells of mixed properties, were beyond the scope and feasibility of the current investigation. It was necessary to first focus directly on domains, domain interaction and their role with regards to cells with multiple preferences before a more complex investigation could proceed.

Though cytochrome oxidase histology was performed on all harvested brain tissue immediately following the terminal procedure (see Section 5.1.2), quality histological sections for analysis was very difficult to achieve, probably due to the long term chronic nature of the experimental procedures. Future experiments could benefit by correlating the penetration location with the recorded electrophysiology as it is possible to project the stripe (thin, thick, or pale) associated with the activity. Previous studies have examined the latencies within and among stripes (Munk et al., 1995); however an achromatic “bar” type stimulus was used in all 
stripes to determine the latency differences. This level of cell categorization by stripe location with our particular stimulus set (color and orientation) may lead to further understanding of latencies across and among stripes.

One particularly useful future experimental adjunct could be the application of domainselective GABA inactivation. Previous studies have shown that pulsed pressure injections of GABA can deliver a highly controlled inactivation of cortical activity. Injections could predictably and temporarily inactivate neural spiking activity at radial distances up to 250 to 500 $\mu \mathrm{m}$ from the injection site. (Hupe et al., 1999). In a limited series of pilot studies GABA inactivation was also successfully used in our lab to briefly inactivate the spiking activity of a cell. In future experiments, a complex stimulus of color and orientation could be presented during the inactivation of a selected domain (e.g., a color preferring domain) and the temporal activity variation would be recorded in an immediately neighboring domain (e.g. an orientation domain. If intra-areal integration is indeed a significant mechanism for integration of color- and orientation-preferring terminals, then one would predict that inactivation of the color-preferring domain would significantly influence the tuning characteristics of some color-oriented neurons in the non-inactivated domain.

Another future experimental adjunct in addition to intrinsic optical imaging could be the utilization of higher resolution functional imaging methods such as 2-photon functional microscopy. This would allow a thorough mapping of neuronal preference for many neurons within V2, and provide comprehensive quantification of the purity of selectivity in the various V2 domains - a measure that is still poorly described. This in vivo experimentally recorded data would provide important new parameters to also improve the biological plausibility of our computer based models. 


\subsection{Conclusions}

This dissertation documents investigations of putative domain interactions in V2 using a cortex-like network implemented via a computer model. This dissertation also documents investigations of the temporal characteristics of anesthetized macaque monkey V2l neurons utilizing in vivo electrophysiological methods. Overall, these experiments specifically investigated the potential for intra-areal mechanisms of visual integration, and in particular putative integrative mechanisms supported by the placement of adjacent and differently preferring functional domains. These investigations focused on the possible neural integration of color and orientation stimulus attributes in V2 via intra-areal connectivity in particular because V2 is well known to functionally organize color-preferring and orientation-preferring neurons into distinct domains and stripe compartments of V2. The modeling results presented here suggest that the striped and clustered functional organization of V2 is more than sufficient for probable and significant integration of color- and orientation-preferring terminal outputs, providing the necessary circuitry framework for the establishment of neurons with mixed colororiented preferences. The electrophysiological results characterized the temporal changes in V2 cell spiking activities following selective stimulation by achromatic-oriented and color-oriented stimulus conditions. The addition of color to an achromatic oriented stimulus lead to an increase in mean latency, increase in mean time to peak firing rate, and a reduction in the initial, transient phase but preserved the later, sustained spiking response. These characteristics suggest a complex temporal evolution of color-orientated preference interaction of color- and orientationpreferring clusters. These results provide novel insight into the potential function of clustered neurons and the temporal evolution of neurons preferring multiple stimulus attributes. Further development of computer modeling incorporating plausible clustered functional organizations, and additional electrophysiological recordings from targeted neurons within a well described functional map may together assist in the ongoing identification and improved understanding of integrative mechanisms in the primate visual system. 


\subsection{References}

Anzai A, Peng X, Van Essen DC (2007) Neurons in monkey visual area V2 encode combinations of orientations. Nat Neurosci 10:1313-1321.

Bednar JA, Kelkar A, Miikkulainen R (2004) Scaling self-organizing maps to model large cortical networks. Neuroinformatics 2:275-302.

Casagrande VA (1999) The mystery of the visual system K pathway. J Physiol 517 (Pt 3):630.

Chen LM, Friedman RM, Roe AW (2005) Optical imaging of SI topography in anesthetized and awake squirrel monkeys. J Neurosci 25:7648-7659.

Chen LM, Turner GH, Friedman RM, Zhang N, Gore JC, Roe AW, Avison MJ (2007) Highresolution maps of real and illusory tactile activation in primary somatosensory cortex in individual monkeys with functional magnetic resonance imaging and optical imaging. $\mathrm{J}$ Neurosci 27:9181-9191.

Cottaris NP, De Valois RL (1998) Temporal dynamics of chromatic tuning in macaque primary visual cortex. Nature 395:896-900.

Douglas RJ, Koch C, Mahowald M, Martin KA, Suarez HH (1995) Recurrent excitation in neocortical circuits. Science 269:981-985.

Elston GN, Rosa MG (1997) The occipitoparietal pathway of the macaque monkey: comparison of pyramidal cell morphology in layer III of functionally related cortical visual areas. Cereb Cortex 7:432-452.

Hendry SH, Reid RC (2000) The koniocellular pathway in primate vision. Annu Rev Neurosci 23:127-153.

Hinkle DA, Connor CE (2005) Quantitative characterization of disparity tuning in ventral pathway area V4. J Neurophysiol 94:2726-2737.

Hubel DH, Livingstone MS (1987) Segregation of form, color, and stereopsis in primate area 18. J Neurosci 7:3378-3415.

Hupe JM, Chouvet G, Bullier J (1999) Spatial and temporal parameters of cortical inactivation by GABA. J Neurosci Methods 86:129-143.

Ito M, Komatsu H (2004) Representation of angles embedded within contour stimuli in area V2 of macaque monkeys. J Neurosci 24:3313-3324.

Johnson EN, Hawken MJ, Shapley R (2004) Cone inputs in macaque primary visual cortex. J Neurophysiol 91:2501-2514. 
Kim DS, Duong TQ, Kim SG (2000) High-resolution mapping of iso-orientation columns by fMRI. Nat Neurosci 3:164-169.

Kiper DC, Fenstemaker SB, Gegenfurtner KR (1997) Chromatic properties of neurons in macaque area V2. Vis Neurosci 14:1061-1072.

Levitt JB, Yoshioka T, Lund JS (1994) Intrinsic cortical connections in macaque visual area V2: evidence for interaction between different functional streams. J Comp Neurol 342:551570.

Livingstone MS, Hubel DH (1984) Anatomy and physiology of a color system in the primate visual cortex. J Neurosci 4:309-356.

Mahadevappa M, Weiland JD, Yanai D, Fine I, Greenberg RJ, Humayun MS (2005) Perceptual thresholds and electrode impedance in three retinal prosthesis subjects. IEEE Trans Neural Syst Rehabil Eng 13:201-206.

Maunsell JH, Gibson JR (1992) Visual response latencies in striate cortex of the macaque monkey. J Neurophysiol 68:1332-1344.

Maunsell JH, Ghose GM, Assad JA, McAdams CJ, Boudreau CE, Noerager BD (1999) Visual response latencies of magnocellular and parvocellular LGN neurons in macaque monkeys. Vis Neurosci 16:1-14.

Munk MH, Nowak LG, Girard P, Chounlamountri N, Bullier J (1995) Visual latencies in cytochrome oxidase bands of macaque area V2. Proc Natl Acad Sci U S A 92:988-992.

Ohki K, Chung S, Ch'ng YH, Kara P, Reid RC (2005) Functional imaging with cellular resolution reveals precise micro-architecture in visual cortex. Nature 433:597-603.

Oram MW, Xiao D, Dritschel B, Payne KR (2002) The temporal resolution of neural codes: does response latency have a unique role? Philos Trans R Soc Lond B Biol Sci 357:987-1001.

Palanker D, Vankov A, Huie P, Baccus S (2005) Design of a high-resolution optoelectronic retinal prosthesis. J Neural Eng 2:S105-120.

Raiguel SE, Lagae L, Gulyas B, Orban GA (1989) Response latencies of visual cells in macaque areas V1, V2 and V5. Brain Res 493:155-159.

Ramsden BM, Hung CP, Roe AW (2001) Real and illusory contour processing in area V1 of the primate: a cortical balancing act. Cereb Cortex 11:648-665.

Roe AW, Ts'o DY (1995) Visual topography in primate V2: multiple representation across functional stripes. J Neurosci 15:3689-3715. 
Roe AW, Ts'o DY (1999) Specificity of color connectivity between primate V1 and V2. J Neurophysiol 82:2719-2730.

Schmolesky MT, Wang Y, Hanes DP, Thompson KG, Leutgeb S, Schall JD, Leventhal AG (1998) Signal timing across the macaque visual system. J Neurophysiol 79:3272-3278.

Shipp S, Zeki S (2002) The functional organization of area V2, I: specialization across stripes and layers. Vis Neurosci 19:187-210.

Sincich LC, Horton JC (2002) Divided by cytochrome oxidase: a map of the projections from V1 to V2 in macaques. Science 295:1734-1737.

VanRullen R, Guyonneau R, Thorpe SJ (2005) Spike times make sense. Trends Neurosci 28:1-4.

Wang Y, Xiao Y, Felleman DJ (2006) V2 Thin Stripes Contain Spatially Organized Representations of Achromatic Luminance Change. Cereb Cortex.

Weiland JD, Greenberg RJ, Humayun MS, Darvas F, Thyerlei D (2005) Clinical Trial of a Prototype Retinal Prosthesis. In: 10th Annual Conference of the International FES Society (Davis. R, Tadej Bajd JB, Warren Grill, Yoichi Shimada and Thomas Sinkjaer., eds). Montreal, Canada.

Xiao Y, Wang Y, Felleman DJ (2003) A spatially organized representation of colour in macaque cortical area V2. Nature 421:535-539.

Yacoub E, Harel N, Ugurbil K (2008) High-field fMRI unveils orientation columns in humans. Proc Natl Acad Sci U S A 105:10607-10612. 


\section{GARRET COOPER \\ 4005 Pinehurst Drive \\ Morgantown, WV 26505 \\ (304) 598-7401 \\ ccooper7@mix.wvu.edu}

\section{EDUCATION:}

2010-2012 M.D. Clinical Training Curriculum

West Virginia University School of Medicine, Morgantown, WV

2005-2010 Ph.D. in Anatomy and Neurobiology

Dissertation Project: The Role of Clustered Organization and Generation of Mixed

Properties in Macaque V2

Dissertation Advisor: Benjamin M. Ramsden, Ph.D.

WVU School of Medicine, Morgantown, WV

2003-2005 M.D. Basic Sciences Curriculum

WVU School of Medicine, Morgantown, WV

1999-2003 B.A. in Cell/Molecular Biology; B.A. in Psychology

Washington and Jefferson College, Washington, PA

\section{RESEARCH EXPERIENCE:}

2005-2010 Graduate Student, Department of Neurobiology and Anatomy

WVU School of Medicine, Morgantown, WV

2002 Howard Hughes Medical Institute Grant apportioned to Washington and Jefferson College

Semester Project: mRNA Expression of Neurodegenerative Diseases

Project Advisor: Bruce H. Wainer, MD, Ph.D.

Emory University, Atlanta, GA

2001 Howard Hughes Medical Institute Grant apportioned to Washington and Jefferson College

Summer Project: mRNA Expression of Neurodegenerative Diseases

Project Advisor: Bruce H. Wainer, MD, Ph.D.

Emory University, Atlanta, GA

\section{TEACHING EXPERIENCE:}

2008-2009 Teaching Assistant, Human Physiology, Department of Neurobiology and Anatomy

Tutor undergraduate students in human physiology and function.

WVU School of Medicine, Morgantown, WV

2006-2008 MD/PhD Admissions Committee

Interview and review applicants for WVU School of Medicine MD/PhD program.

WVU School of Medicine, Morgantown, WV

2000-2003 Teaching Assistant, General Biology, Department of Biology

Assist undergraduate students in understanding the concepts and details of the General

Biology Lecture and direct study sessions with the aid of lecture based worksheets.

Washington and Jefferson College, Washington, PA

2000-2003 Writing Mentor, General Biology, Department of Biology 
Aid undergraduate students in developing the necessary writing skills and techniques. Washington and Jefferson College, Washington, PA

\section{HONORS AND AWARDS:}

2003 - Delta Tau Delta International Fraternity, Almunus

2000-2003 - Washington and Jefferson Dean's List

1999-2003 - Washington and Jefferson Howard J. Burnett Memorial Presidential Scholarship

2001-2002 - Phi Sigma Biology Honorary

2001-2002 - Alpha Psi Omega Theatre Honorary

2001-2002 - Order of Omega Fraternity Honorary

2001-2002 - Gamma Sigma Alpha National Greek Academic Honorary

1997 - Boy Scouts of America, Eagle Scout with Golden Palm

MEMBERSHIP IN PROFESSIONAL SOCIETIES:

2008-2009 Vision Sciences Society

2006-2009 Society for Neuroscience

\section{PUBLICATIONS:}

Cooper CG, Ramsden, BM. Clustered cortical organization and the enhanced probability of intra-areal functional integration. (in press)

Cooper CG, Ramsden, BM. Sensory integration and temporal response modulation in macaque Area V2: Single Unit Electrophysiology. (in preparation)

\section{PUBLISHED ABSTRACTS AND PRESENTATIONS:}

Cooper CG (2009). The Role of Clustered Organization and Generation of Mixed Properties in Macaque V2 [Oral Presentation]. Dissertation Defense Seminar, WVU School of Medicine, Morgantown, WV.

Cooper CG, Ramsden, BM (2008). Sensory integration and temporal response modulation in macaque Area V2: intra-areal mechanisms? [Poster]. Program No. 666.22. 2008 Neuroscience Meeting Planner. Washington, DC: Society for Neuroscience, 2008.

Cooper CG (2008). The temporal responses of macaque V2 neurons to colored and oriented visual stimuli. [Oral Presentation]. Dissertation Proposal Seminar, WVU School of Medicine, Morgantown, WV.

Cooper CG, Ramsden BM (2008). Color modulation of temporal response to oriented stimulation in macaque V2 [Abstract]. Journal of Vision, 8(6):359

Cooper CG, Ramsden BM (2008). Color modulation of temporal response to oriented 
stimulation in macaque V2. [Poster]. Vision Sciences Society Meeting, Sarasota, FL.

Cooper CG, Ramsden BM (2008). Temporal response modulations of Macaca fascicularis V2 neurons to color and orientation. [Poster]. Van Liere Convocation and Research Day, WVU Health Sciences Center, Morgantown, WV.

Cooper CG, Ramsden BM (2007). Color meets orientation: cluster organization probabilities in Area V2 of macaque visual cortex. [Poster]. WVU Center for Neuroscience Retreat, Oglebay Resort and Conference Center, Wheeling, WV.

Cooper CG, Ramsden BM (2007). Color meets orientation: cluster organization probabilities in Area V2 of macaque visual cortex. [Poster]. Van Liere Convocation and Research Day, WVU Health Sciences Center, Morgantown, WV.

Cooper CG (2007). Color Organization in Macaque Primary Visual Cortex: An Analytical Review of the Past and Current Research. [Oral Presentation]. Qualifying Seminar, WVU School of Medicine, Morgantown, WV.

Cooper CG, Ramsden BM (2006). Color meets orientation: cluster organization probabilities in area V2 of macaque visual cortex. [Poster]. Program No. 546.10. 2006 Neuroscience Meeting Planner. Atlanta, GA: Society for Neuroscience, 2006.

Cooper CG, Ramsden BM (2006). Geometric implications of cluster juxtaposition on neuronal stimulus preference in macaque V2 cortex. [Poster]. WVU Center for Neuroscience Retreat, Nemacolin Woodlands Resort, Laurel Highlands, PA.

Ramsden BM, Cooper CG (2006). Does map adjacency contribute to neuronal response construction in V2? [Abstract]. Journal of Vision, 6(6):893

Ramsden BM, Cooper CG (2006). Does map adjacency contribute to neuronal response construction in V2? [Poster]. Vision Sciences Society Meeting, Sarasota, FL. 